\title{
Nuclear Modification of $J / \psi$ and Drell-Yan Production at the E906/SeaQuest Experiment
}

\author{
by \\ Catherine Ayuso \\ A dissertation submitted in partial fulfillment \\ of the requirements for the degree of \\ Doctor of Philosophy \\ (Applied Physics) \\ in The University of Michigan \\ 2020
}

\section{Doctoral Committee:}

Associate Professor Christine Aidala, Chair

Professor Cagliyan Kurdak

Professor Wolgang Lorenzon

Associate Professor Thomas Schwarz 


\section{Catherine Ayuso}

cayuso@umich.edu

ORCID iD: 0000-0002-4825-1218

(c) Catherine Ayuso 2020 


\section{DEDICATION}

to The Void 


\section{ACKNOWLEDGEMENTS}

My most profound gratitude for their unrelenting heroism, their sacrifices, their love and support goes out, first and foremost, to my parents, Rita and Francisco. They uprooted from their country at the peak of both of their careers but at a time when a safe and fertile future for their children seemed entirely unimaginable. My mother was an entrepreneur with her own business, my father, a thriving industrial engineer. We lived comfortably, surrounded by the warmth of our family. We were happy, even as the bombs fell far too casually around different sectors of Bogota and as the guerrilla kidnappings became a permanent fixture in the night-time news. Their choice was clear-they needed to make a new home for us. Once in the states, they fought to make a space for themselves and for us in a country that did not want us. In a country that did not value their professional backgrounds and expertise. In a country that turned its back on us when we spoke with an accent, when we celebrated our customs, when we did not acquiesce to theirs. Nevertheless, they persisted with a fierce and creative spirit, one that to this day, has served as my most treasured source of mentorship. They are warriors. And I owe them far more than what I could fit in the pages of a thesis acknowledgment.

There are a few amazing women that have helped shape me into the person that I am today. My sister, Maria Jose, who is a true interdisciplinary maven that has taught me the importance of making time and space to develop your artistic craft and feed your passions, alongside the pursuit of higher education in science. My best friend, Kiki, who is my partner-in-crime and has been a beacon of light in some of the darkest moments of my life. My mother, who is a visionary and has managed to reinvent herself time and time again professionally, personally, spiritually and remains the strongest pillar of support and 
greatest source of inspiration for my sister and myself.

I'd like to express my sincerest gratitude to my advisor, Christine Aidala, for her guidance and her humanity throughout this journey. Her rigorous advising was never absent of warmth, patience and understanding. Throughout the 4 years of working together, she always created a safe space for me to explore new ideas and learn from challenges without fearing mistakes. She is not only a wonderful mentor but a true inspiration for all us who have had the pleasure of working with her.

I am also very grateful for the support and mentorship of several of my colleagues. Bryan Ramson helped show me the ropes around SeaQuest, as a new graduate student, and was a fantastic person to collaborate with and share the title of target expert. Nicole Lewis quickly became my favorite conference buddy and the best person to share a temporary office with during my visits to Ann Arbor. Jason Dove helped me an innumerable amount of times with software and coding related issues with thoroughness, kindness and patience. But I am especially thankful to my colleague and friend, Arun Tadepalli, for being an unyielding support system and an invaluable resource for all experiment-related matters, particularly during the last stages of my Ph.D. He always made himself available to discuss physics, to resolve analysis issues together or to simply share a laugh and breather in between the austerity of graduate school.

To Paul Reimer, Don Geesaman and the rest of the SeaQuest family, thank you for all the lessons learned, for all the guidance, for the experience of working together effectively and collaboratively to address so many complex challenges from the experiment. Thank you for always having a kind, encouraging word and invaluable advice to share with me. I am lucky to have walked my graduate school journey alongside you all. 


\section{TABLE OF CONTENTS}

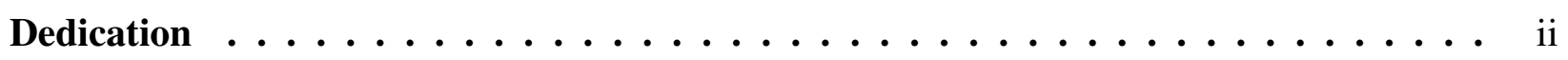

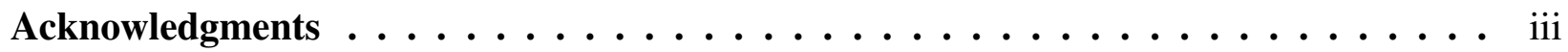

List of Tables ............................. $\quad \ldots$

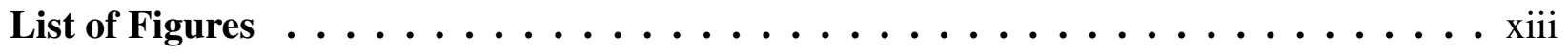

Abstract ..............................

Chapter 1: Introduction .......................... 1

1.1 The Standard Model . . . . . . . . . . . . . . . . . . . 1

1.2 Quantum Chromodynamics . . . . . . . . . . . . . . . . 3

1.3 The Drell-Yan process . . . . . . . . . . . . . . 7

$1.4 J / \psi$ production $\ldots \ldots \ldots \ldots \ldots \ldots$

1.5 Process kinematics at SeaQuest . . . . . . . . . . . . . . . . 13

1.6 Initial and final-state effects . . . . . . . . . . . . . 16

1.6.1 Parton energy loss . . . . . . . . . . . . . . 18

1.6.2 $J / \psi$ production suppression: CNM effect and QGP probe . . . . . . . . . 20

1.7 Previous measurements of nuclear modification $\ldots \ldots \ldots \ldots$

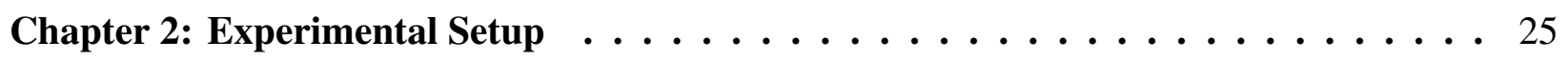


2.1 Proton beam production . . . . . . . . . . . . . . . . . . . . . 27

$2.1 .1 \quad$ Beam structure $\ldots \ldots \ldots \ldots \ldots$

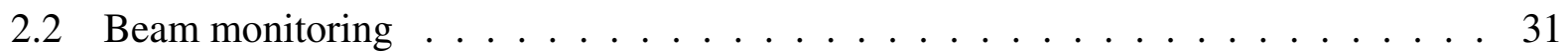

2.2 .1 Beam intensity monitor . . . . . . . . . . . . . . . . 31

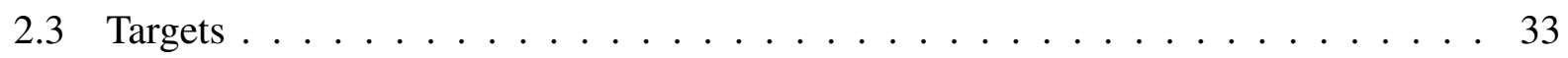

2.3 .1 Solid targets . . . . . . . . . . . . . . . . . . . . . 34

2.3.2 Cryogenic targets . . . . . . . . . . . . . . . . 35

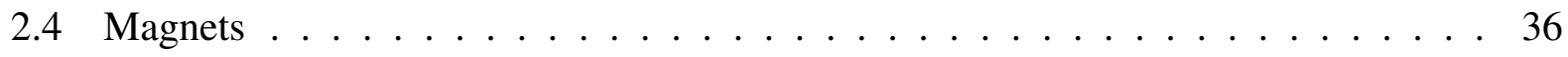

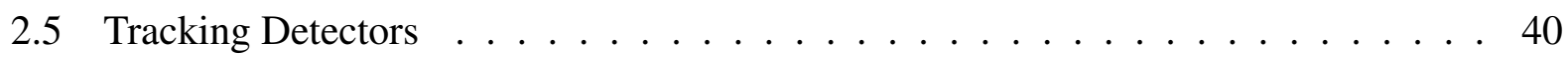

2.5 .1 Hodoscopes . . . . . . . . . . . . . . . . . . . . . 40

2.6 Drift chambers . . . . . . . . . . . . . . . . . 43

2.6.1 Drift chamber readout electronics . . . . . . . . . . . . . . . 49

2.7 Proportional tubes . . . . . . . . . . . . . . . . . . . 51

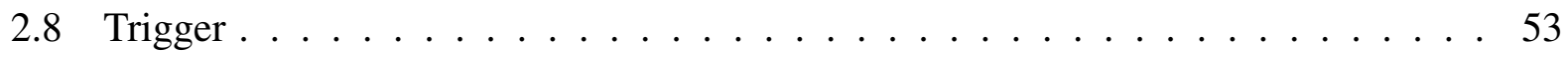

2.9 Data acquisition $(\mathrm{DAQ}) \ldots \ldots \ldots \ldots$

2.9 .1 MainDAQ . . . . . . . . . . . . . . . . . 56

$2.9 .2 \quad$ ScalerDAQ $\ldots \ldots \ldots \ldots \ldots \ldots$

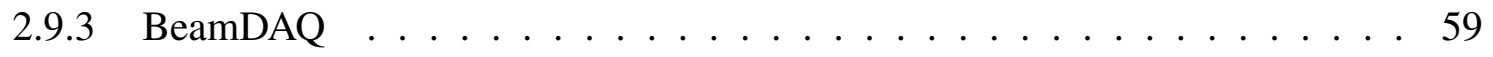

2.9 .4 Slow controls $\ldots \ldots \ldots \ldots \ldots$

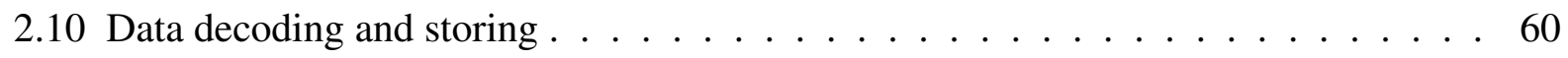

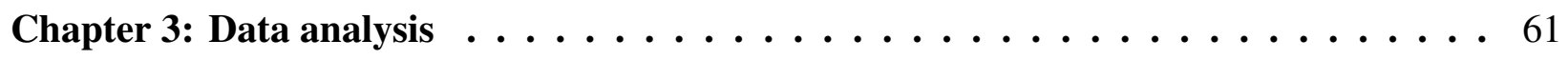

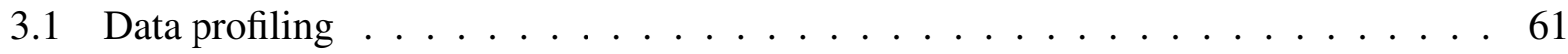


3.2 Reconstructing dimuons . . . . . . . . . . . . . . . . . . . 62

3.2.1 Pre-tracking data trimming methods $\ldots \ldots \ldots \ldots 2$

3.2.2 Reconstructing tracklets . . . . . . . . . . . . . . . . . . . 64

3.2.3 Reconstructing global tracks . . . . . . . . . . . . . . . 67

3.2 .4 Vertex reconstruction . . . . . . . . . . . . . . . . . . . . . 69

3.2.5 Dimuon vertex reconstruction $\ldots \ldots \ldots \ldots$

3.3 Monte Carlo simulations . . . . . . . . . . . . . . . . . . 71

3.3 .1 Dimuon generator . . . . . . . . . . . . . . . . . . . . 72

3.3.2 Background generators . . . . . . . . . . . . . . . . 73

3.3 .3 MC simulation formats . . . . . . . . . . . . . . . 73

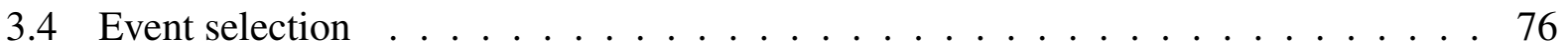

$3.4 .1 \quad$ Spill level cuts $\ldots \ldots \ldots \ldots \ldots \ldots \ldots$

3.4 .2 Event level cuts . . . . . . . . . . . . . . . . . . . . . 77

3.4 .3 Track level cuts . . . . . . . . . . . . . . . . . . . . . 77

3.4 .4 Dimuon level cuts . . . . . . . . . . . . . . . . . . . . . . . . 80

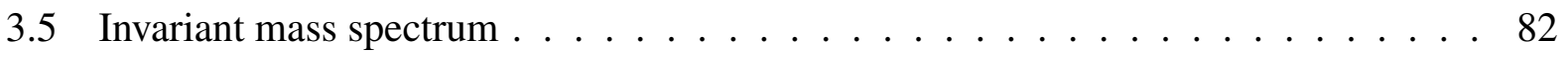

3.6 Normalized yields for different kinematic variables $\ldots \ldots$. . . . . . . . 83

$3.6 .1 \quad J / \psi$ plots of normalized yields $\ldots \ldots \ldots \ldots \ldots$

3.6.2 DY plots of normalized yields . . . . . . . . . . . . . . 90

3.7 Liquid target corrections $\ldots \ldots \ldots \ldots \ldots$

3.8 Cross-section ratios $\ldots \ldots \ldots \ldots$

3.8 .1 Statistical errors . . . . . . . . . . . . . . . . . . . . 99

3.9 The Intensity-Extrapolation (IE) Method ～. . . . . . . . . . . . . . . . 100 
3.9.1 Logic behind Fit function . . . . . . . . . . . . . . . . . . . . 101

3.9.2 Fit function for intensity dependence plots . . . . . . . . . . . . 102

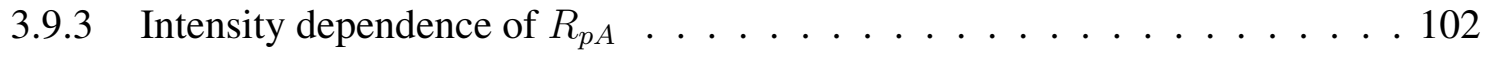

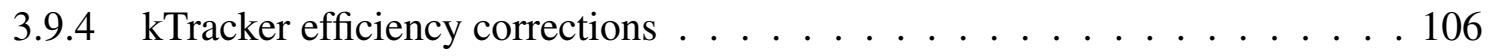

3.9.5 Physics contamination factor $F$ for $J / \psi$ signal . . . . . . . . . . 107

Chapter 4: Results . . . . . . . . . . . . . . . . . . . . . . . . . . 109

4.1 Nuclear modification of $J / \Psi$ and Drell-Yan pairs $\ldots \ldots$. . . . . . . . 109

$4.1 .1 \quad$ Discussion . . . . . . . . . . . . . . . . . . . . . . . . 109

4.2 Sources of systematic uncertainty . . . . . . . . . . . . . . . 113

4.2.1 $p_{T}$ systematic error for $J / \psi$ and Drell-Yan processes $\ldots \ldots \ldots \ldots$

4.2.2 $x_{F}$ systematic error for $J / \psi$ and Drell-Yan processes $\ldots \ldots \ldots \ldots$

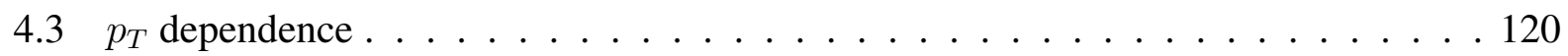

$4.3 .1 \quad p_{T}$ dependence results for $J / \psi \ldots \ldots \ldots \ldots \ldots \ldots \ldots \ldots$

4.3.2 $p_{T}$ dependence results for Drell-Yan $\ldots \ldots \ldots \ldots \ldots \ldots \ldots$

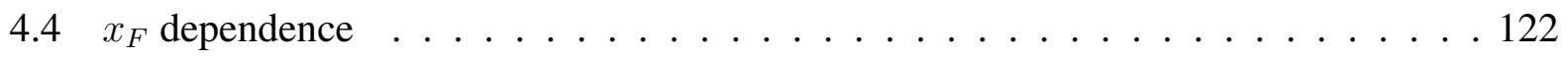

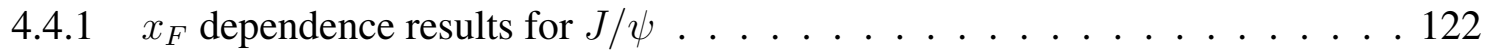

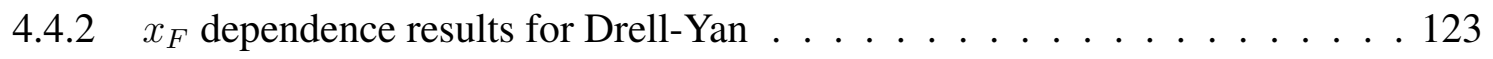

4.5 Nuclear dependence comparisons . . . . . . . . . . . . . . . . . . . 123

Chapter 5: Summary . . . . . . . . . . . . . . . . . 130

5.1 Nuclear modification $\ldots \ldots \ldots 130$

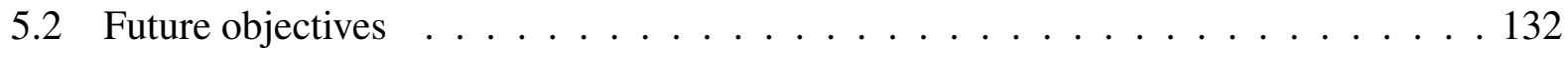

$5.2 .1 \quad$ More data $! \ldots \ldots \ldots \ldots . \ldots \ldots \ldots$ 
5.2 .2 Systematic uncertainties improvement . . . . . . . . . . . . . . 132

5.2.3 Nuclear dependence for different target bases: $\mathrm{C}$ and $\mathrm{LH}_{2} \quad \ldots \ldots \ldots 2$

Appendix A: Raw yields and intensity dependence plots . . . . . . . . . . . 135

A.1 Raw dimuon yield tables $\ldots \ldots \ldots 135$

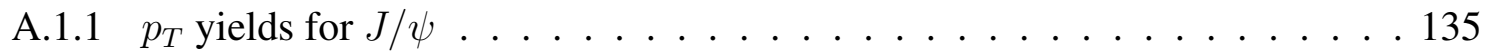

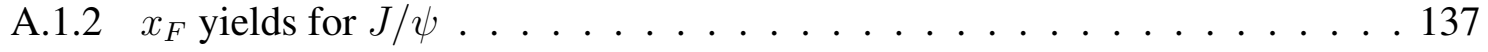

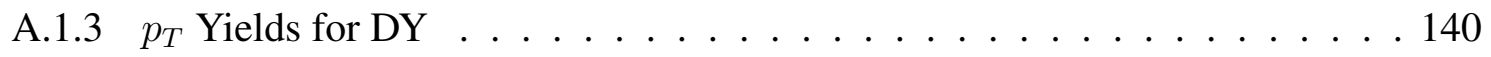

A.1.4 $x_{F}$ Yields for DY . . . . . . . . . . . . . . . . . . . 142

A.2 $R_{p A}$ intensity dependence plots $\ldots \ldots \ldots \ldots \ldots \ldots \ldots \ldots$

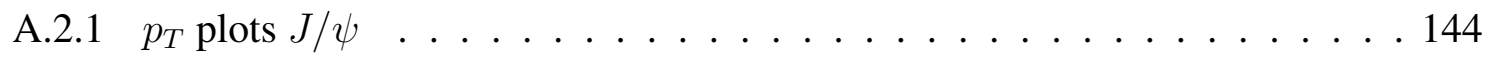

A.2.2 $p_{T}$ plots Drell-Yan . . . . . . . . . . . . . . . . 147

A.2.3 $x_{F}$ plots $J / \psi \ldots \ldots \ldots \ldots \ldots \ldots$

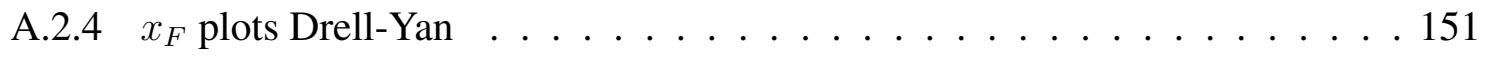

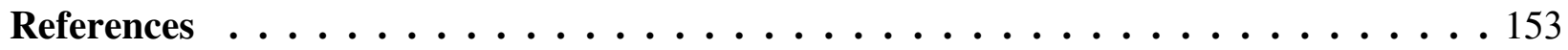




\section{LIST OF TABLES}

\section{TABLE}

2.1 Features of the targets at SeaQuest. The "Spills/Cycle" values shown represent default configurations. Values can change depending on the response to sample balancing needs and running configurations [29]. . . . . . . . . . . . . 33

2.2 Information on different hodoscope planes. The designation (L) and (R) refer to beam left or right and (T) and (B) refers to Top and Bottom. $\mathrm{Z}$ - position is measured from the front face of FMAG. [18] . . . . . . . . . . . . . . . . . 41

2.3 Drift chamber specifications from [29]. . . . . . . . . . . . . . . 45

2.4 Configurations of Drift chambers used in different Runs [18] . . . . . . . . . . . 46

2.5 Proportional tubes settings. . . . . . . . . . . . . . . 53

2.6 Settings for the different SeaQuest triggers. For FPGA 5, $p_{x}>3 \mathrm{GeV} / \mathrm{c}$ is an additional requisite $[29] . \ldots \ldots \ldots \ldots$

3.1 Specifications of roadsets. . . . . . . . . . . . . . . . 61

3.2 "Good" run and spill ranges. . . . . . . . . . . . . . . . . 62

3.3 Cuts on the number of detector hits [4] [18]. . . . . . . . . . 65

3.4 "Good spill" specifications from $[4] \ldots \ldots \ldots \ldots$

3.5 Raw proton weighted average quantities for full data set. . . . . . . . . . . . 98

3.6 $F_{A}$ factor values for each target type across different intensity bins. Values presented are percentages. . . . . . . . . . . . . . . . . . 107

$4.1 \mathrm{~J} / \psi R_{p A}$ systematic error per $p_{T}$ bin $(\mathrm{GeV} / \mathrm{c})$ for carbon $\ldots \ldots \ldots$

$4.2 \mathrm{~J} / \psi R_{p A}$ systematic error per $p_{T}$ bin $(\mathrm{GeV} / \mathrm{c})$ for iron $\ldots \ldots \ldots$ 
$4.3 J / \psi R_{p A}$ systematic error per $p_{T}$ bin $(\mathrm{GeV} / \mathrm{c})$ for tungsten $\ldots \ldots \ldots \ldots$

4.4 DY $R_{p A}$ systematic error per $p_{T}$ bin $(\mathrm{GeV} / \mathrm{c})$ for carbon $\ldots \ldots \ldots \ldots$

4.5 DY $R_{p A}$ systematic error per $p_{T}$ bin $(\mathrm{GeV} / \mathrm{c})$ for iron $\ldots \ldots \ldots 117$

4.6 DY $R_{p A}$ systematic error per $p_{T}$ bin $(\mathrm{GeV} / \mathrm{c})$ for tungsten $\ldots \ldots \ldots 117$

$4.7 \quad J / \psi R_{p A}$ systematic error per $x_{F}$ bin for carbon $\ldots \ldots \ldots \ldots \ldots \ldots$

$4.8 J / \psi R_{p A}$ systematic error per $x_{F}$ bin for iron $\ldots \ldots \ldots \ldots \ldots \ldots$

$4.9 J / \psi R_{p A}$ systematic error per $x_{F}$ bin for tungsten $\ldots \ldots \ldots \ldots \ldots$

4.10 DY $R_{p A}$ systematic error per $x_{F}$ bin for carbon $\ldots \ldots \ldots 119$

4.11 DY $R_{p A}$ systematic error per $x_{F}$ bin for iron . . . . . . . . . . . . 119

4.12 DY $R_{p A}$ systematic error per $x_{F}$ bin for tungsten $\ldots \ldots \ldots \ldots$

A.1 Dimuon yields per intensity bin for $0.0<p_{T}<0.33 \ldots \ldots \ldots$

A.2 Dimuon yields per intensity bin for $0.33<p_{T}<0.54 \ldots \ldots \ldots \ldots \ldots$

A.3 Dimuon yields per intensity bin for $0.54<p_{T}<0.72 \ldots \ldots \ldots \ldots \ldots$

A.4 Dimuon yields per intensity bin for $0.72<p_{T}<0.99 \ldots \ldots \ldots \ldots \ldots$

A.5 Dimuon yields per intensity bin for $0.99<p_{T}<2.3 \ldots \ldots \ldots \ldots$

A.6 Dimuon yields per intensity bin for $0.0<x_{F}<0.5 \ldots \ldots \ldots \ldots$

A.7 Dimuon yields per intensity bin for $0.5<x_{F}<0.6 \ldots \ldots \ldots \ldots$

A.8 Dimuon yields per intensity bin for $0.6<x_{F}<0.7 \ldots \ldots \ldots \ldots$

A.9 Dimuon yields per intensity bin for $0.7<x_{F}<0.8 \ldots \ldots \ldots$. . . . . . 139

A.10 Dimuon yields per intensity bin for $0.8<x_{F}<0.95 \ldots \ldots \ldots$

A.11 Dimuon yields per intensity bin for $0.0<p_{T}<0.33 \ldots \ldots \ldots \ldots$

A.12 Dimuon yields per intensity bin for $0.33<p_{T}<0.54 \ldots \ldots \ldots \ldots \ldots$ 
A.13 Dimuon yields per intensity bin for $0.54<p_{T}<0.72 \ldots \ldots \ldots \ldots$

A.14 Dimuon yields per intensity bin for $0.72<p_{T}<0.99 \ldots \ldots \ldots \ldots$

A.15 Dimuon yields per intensity bin for $0.99<p_{T}<2.3 \ldots \ldots$. . . . . . . . 142

A.16 Dimuon yields per intensity bin for $0.0<x_{F}<0.5 \ldots \ldots . \ldots$. . . . . . 142

A.17 Dimuon yields per intensity bin for $0.5<x_{F}<0.6 \ldots \ldots \ldots$

A.18 Dimuon yields per intensity bin for $0.6<x_{F}<0.7 \ldots \ldots \ldots$

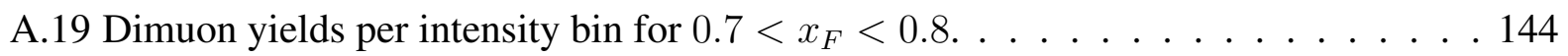

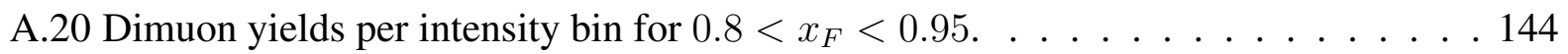




\section{LIST OF FIGURES}

FIGURE

1.1 The Standard Model of particle physics $[1] \ldots \ldots \ldots$

1.2 The dependence of QED and QCD coupling constants to $Q^{2}[4] \ldots \ldots$

1.3 Diagram of DY process $\ldots \ldots \ldots \ldots \ldots \ldots$

1.4 DY cross-section as a function of the invariant mass of muon pairs [8] $\ldots \ldots .8$

1.5 Partonic diagrams of $J / \psi$ production $\ldots \ldots \ldots \ldots$

1.6 Ratio of quark annihilation and gluon fusion cross-sections for $c \bar{c}$ production in p-p collisions to their sum vs. $x_{F}$ at varying $E_{c m}=\sqrt{s} . E_{c m}$ at SeaQuest is $15.4 \mathrm{GeV}$.

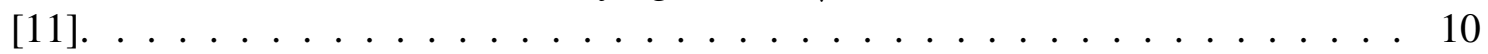

1.7 Parton distributions as a function of $\mathrm{x}$ with $Q^{2}=M_{J / \psi}{ }^{2}[11] \ldots \ldots \ldots 11$

1.8 Differential cross-sections as a function of $x_{F}$ at $120 \mathrm{GeV}$ beam energy. The red curve relates to the contribution from $q \bar{q}$ annihilation and the blue curve from gluon-gluon fusion $[13] . \ldots \ldots \ldots \ldots \ldots$

1.9 Differential cross-sections as a function of $x_{F}$ at $800 \mathrm{GeV}$ beam energy. The red curve relates to the contribution from $q \bar{q}$ annihilation and the blue curve from gluon-gluon fusion $[14,13] . \ldots \ldots \ldots \ldots \ldots$

1.10 Collins-Soper frame is the center of mass frame of the dileptons produced in hadron-hadron collisions. $\theta_{C S}$ is the polar angle and $\phi_{C S}$ is the azimuthal angle [15]. . . . . . . . . . . . . . . . . . . . . 14

$1.11 x_{\text {beam }}$ vs. $x_{\text {target }}$ plot with simulated SeaQuest populated acceptance (top). LO parton distributions as a function of $\mathrm{x}$-Bjorken (bottom) [16] . . . . . . . . . 16

1.12 Diagram of initial and final-state effects. . . . . . . . . . . . . 17

1.13 Energy loss process at SeaQuest $[18] \ldots \ldots \ldots$ 
1.14 Illustration of why the yield ratio, $R_{p A}$, drops as a function of $x_{F}[4] \ldots \ldots$

1.15 The $J / \psi$ survival probability as a function of energy density for suppression by deconfinement and by hadronic absorption (top). Sequential quarkonium suppression, where $1 \mathrm{~S}$-state is $J / \psi$ (bottom) $[20,21] \ldots \ldots \ldots \ldots$. . . . . . . . 21

1.16 Model predictions for the $J / \psi$ nuclear suppression factor compared to the E866 data for yield ratios, $R_{F e / B e}\left(p_{T}\right)$ and $R_{W / B e}\left(p_{T}\right)$, in the intermediate and large- $x_{F}$. The dashed lines indicate the effect of momentum broadening only [24, 25] . . . . 22

1.17 Model predictions for the $J / \psi$ nuclear suppression factor $R_{p A}\left(p_{T}\right)$ (yield ratio) in minimum bias d-Au collisions at RHIC, at backward (top) and forward (bottom) rapidities. The dashed lines indicate the effect of momentum broadening only [24, 22]

1.18 Yield ratios, $R_{F e / B e}$ and $R_{W / B e}$, for DY events versus dimuon mass, $x_{2}$ (similar to $\left.x_{\text {target }}\right), x_{F}$ and $x_{1}$ (similar to $x_{\text {beam }}$ ). Solid circles are results from E866 and open circles are results from its predecessor Fermilab experiment, E772. The solid curves are the predicted cross-section ratios for E866, integrated over the other variables from LO-calculations using EKS98 and MRST [26, 27].

2.1 The SeaQuest Spectrometer [29] . . . . . . . . . . . . . . . . 26

2.2 Fermilab Accelerator Complex [30] . . . . . . . . . . . . . . . . . 29

2.3 Micro structure of the beam along with varying intensity buckets from [4]. . . . . 30

2.4 The Beam Intensity Monitor (BIM) Čerenkov counter. [29] . . . . . . . . . . . . . 32

2.5 The beam intensity measured by the Beam DAQ Cerenkov counter every beam bucket. Each strip shows the number of protons per beam bucket as a function of time. The red line in each plot denotes the threshold above which the trigger is inhibited. [29] . . . . . . . . . . . . . . . . . 33

2.6 Diagrammatic layout of mobile target table showing all 7 SeaQuest targets (top

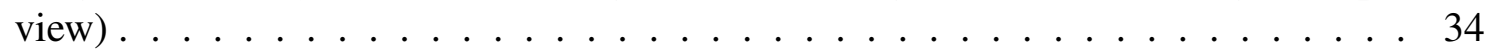

2.7 Schematic depiction of the flask and vacuum plumbing for the two cryogenic targets [29]. . . . . . . . . . . . . . . . . . . . 36

2.8 FMAG schematic view. . . . . . . . . . . . . . . 38

2.9 KMAG schematic view. . . . . . . . . . . . . . . . . . 39 
2.10 Diagram of hodoscopes and its features. . . . . . . . . . . . 40

2.11 SeaQuest Hodoscopes . . . . . . . . . . . . . . . . . . . . . . . . 42

2.12 Drop-like shape of an avalanche. A cloud chamber picture (left) . A schematic

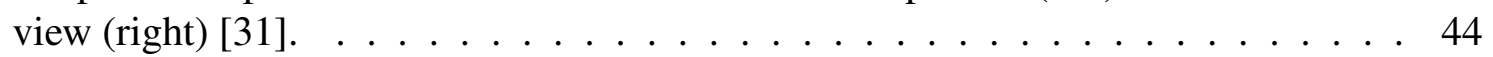

2.13 Diagram of drift time calculation. . . . . . . . . . . . . . . . . . 45

2.14 Depiction of DC1.1 + DC1.2 configuration. . . . . . . . . . . . 47

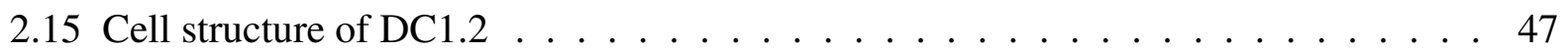

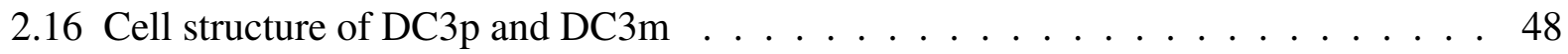

2.17 Picture of an ASDQ card along with ribbon cables, ferrites (used for noise suppression) and cable supports . . . . . . . . . . . . . . . . . . . . . . . 49

2.18 Level Shifter Boards _ . . . . . . . . . . . . . . . . . . . . 50

2.19 Common stop mode of a TDC $\ldots \ldots \ldots \ldots$

2.20 Proportional tubes schema $[29] \ldots \ldots \ldots 2$

2.21 Function structure of the NIM3 pseudo-random trigger. . . . . . . . . . . . . 54

2.22 SeaQuest trigger diagram $[32] \ldots \ldots \ldots \ldots \ldots \ldots$

2.23 Trigger distribution schema. Readout is asynchronous [4] $\ldots \ldots \ldots$

2.24 Workflow of the MainDAQ $[18] \ldots \ldots \ldots \ldots \ldots$

3.1 Flowchart for kTracker from $[4] \ldots \ldots \ldots$

3.2 Cluster removal flowchart $[35] \ldots \ldots$. . . . . . . . . . . . . . . . . . 64

3.3 Reconstructing drift chamber tracklets $[36] . \ldots \ldots \ldots \ldots 6 \ldots$

3.4 Track projection via the sagitta method from [36]. . . . . . . . . . . . . 67

3.5 Geometric depiction of the Kalman filter process [36]. . . . . . . . . . . . 68

3.6 Workflow of the Kalman filter process [36]. . . . . . . . . . . . . . . 69 
3.7 Procedure for muon tracks through a slice of FMAG from [36] . . . . . . . . . 69

3.8 Vertex position for a set Z-position (dotted line) and mass [36]. . . . . . . . . 71

3.9 Kinematic distributions of the MC events for two physics processes. . . . . . . . 74

3.10 Kinematic distributions of the MC events for two physics processes. . . . . . . . 75

3.11 An invariant mass spectrum plot for carbon. Values on the upper right hand corner (nDY, nFlask...) are normalization values for each component provided by the fit. The dotted line indicates where the lower mass cut takes place for DY events. . . . 83

$3.12 \mathrm{~J} / \psi$ normalized yields for $x_{B}$ : (a) $\mathrm{LH}_{2}$, Empty flask and $\mathrm{LD}_{2}$ and b) No target, $\mathrm{Fe}$, $\mathrm{C}$ and $\mathrm{W}$ targets. . . . . . . . . . . . . . . . . . . 84

$3.13 \mathrm{~J} / \psi$ normalized yields for $x_{F}$ : (a) $\mathrm{LH}_{2}$, Empty flask and $\mathrm{LD}_{2}$ and b) No target, $\mathrm{Fe}$, $\mathrm{C}$ and $\mathrm{W}$ targets. . . . . . . . . . . . . . . . . . 85

$3.14 \mathrm{~J} / \psi$ normalized yields for $p_{T}$ : (a) $\mathrm{LH}_{2}$, Empty flask and $\mathrm{LD}_{2}$ and b) No target, $\mathrm{Fe}$, $\mathrm{C}$ and $\mathrm{W}$ targets. . . . . . . . . . . . . . . . . . . 86

$3.15 \mathrm{~J} / \psi$ normalized yields for $d p z$ : (a) $\mathrm{LH}_{2}$, Empty flask and $\mathrm{LD}_{2}$ and b) No target, $\mathrm{Fe}, \mathrm{C}$ and $\mathrm{W}$ targets. . . . . . . . . . . . . . . . . 87

$3.16 \mathrm{~J} / \psi$ normalized yields of $d z$ (dimuon vertex) for (a) $\mathrm{LH}_{2}$, Empty flask and $\mathrm{LD}_{2}$ and b) No target, Fe, $\mathrm{C}$ and $\mathrm{W}$ targets. . . . . . . . . . . . . . 88

$3.17 \mathrm{~J} / \psi$ normalized yields for $\cos \theta$ : (a) $\mathrm{LH}_{2}$, Empty flask and $\mathrm{LD}_{2}$ and b) No target, $\mathrm{Fe}, \mathrm{C}$ and $\mathrm{W}$ targets. . . . . . . . . . . . . . . . . . . 89

3.18 DY normalized yields for $x_{B}$ : (a) $\mathrm{LH}_{2}$, Empty flask and $\mathrm{LD}_{2}$ and b) No target, $\mathrm{Fe}$, $\mathrm{C}$ and $\mathrm{W}$ targets. . . . . . . . . . . . . . . . . . 90

3.19 DY normalized yields for $x_{F}$ : (a) $\mathrm{LH}_{2}$, Empty flask and $\mathrm{LD}_{2}$ and b) No target, $\mathrm{Fe}$, $\mathrm{C}$ and $\mathrm{W}$ targets. . . . . . . . . . . . . . . . . . 91

3.20 DY normalized yields for $p_{T}$ : (a) $\mathrm{LH}_{2}$, Empty flask and $\mathrm{LD}_{2}$ and b) No target, $\mathrm{Fe}$,

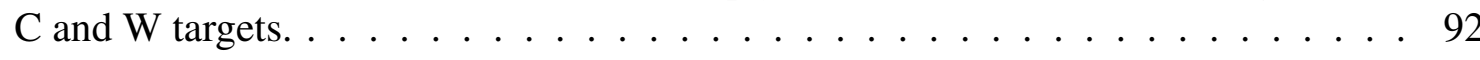

3.21 DY normalized yields for $d p z$ : (a) $\mathrm{LH}_{2}$, Empty flask and $\mathrm{LD}_{2}$ and b) No target, $\mathrm{Fe}$, $\mathrm{C}$ and $\mathrm{W}$ targets. . . . . . . . . . . . . . . . 93

3.22 DY normalized yields of $d z$ (dimuon vertex) for (a) $\mathrm{LH}_{2}$, Empty flask and $\mathrm{LD}_{2}$ and b) No target, Fe, C and $\mathrm{W}$ targets. . . . . . . . . . . . . . . . . . . . 94 
3.23 DY normalized yields for $\cos \theta$ : (a) $\mathrm{LH}_{2}$, Empty flask and $\mathrm{LD}_{2}$ and b) No target, $\mathrm{Fe}, \mathrm{C}$ and $\mathrm{W}$ targets. . . . . . . . . . . . . . . . . . . . . . . . 95

3.24 Intensity dependence of $J / \psi R_{p A}$ for carbon for different $p_{T}(\mathrm{GeV} / \mathrm{c})$ bins. $F=$ $p 0+p 1 *$ intensity $+p 2 *$ intensity ${ }^{2}$ fit was used for the extrapolation, with $p 0$ taken as the nominal $R_{p A}$ value. The fit parameters $p 1$ and $p 2$ are common for all fits. Plots for iron and tungsten can be found in Appendix A.2. . . . . . . . . . . 103

3.25 Intensity dependence of DY $R_{p A}$ for carbon for different $p_{T}(\mathrm{GeV} / \mathrm{c})$ bins. $F=$ $p 0+p 1 *$ intensity $+p 2 *$ intensity ${ }^{2}$ fit was used for the extrapolation, with $p 0$ taken as the nominal $R_{p A}$ value. The fit parameters $p 1$ and $p 2$ are common for all fits. Plots for iron and tungsten can be found in Appendix A.2. . . . . . . . . . . 104

$3.26 \mathrm{~J} / \psi R_{p A}$ for carbon for different $x_{F}$ bins. $F=p 0+p 1 * i n t e n s i t y+p 2 *$ intensity $^{2}$ fit was used for the extrapolation. The fit parameters $p 1$ and $p 2$ are common for all fits. Plots for iron and tungsten can be found in Appendix A.2. . . . . . . . . . . 105

3.27 DY $R_{p A}$ for carbon for different $x_{F}$ bins. $F=p 0+p 1 *$ intensity $+p 2 *$ intensity $^{2}$ fit was used for the extrapolation. The fit parameters $p 1$ and $p 2$ are common for all fits. Plots for iron and tungsten can be found in Appendix A.2 . . . . . . . . . . 106

4.1 $R_{p A}$ vs $p_{T}(\mathrm{GeV} / \mathrm{c})$ for carbon, iron and tungsten for $J / \psi$. Boxes around points represent systematic error. . . . . . . . . . . . . . . . . . . . . 120

4.2 $R_{p A}$ vs $p_{T}(\mathrm{GeV} / \mathrm{c})$ for carbon, iron and tungsten for DY. Boxes around points represent systematic error. . . . . . . . . . . . . . . . . . 121

4.3 $R_{p A}$ vs $x_{F}$ for carbon, iron and tungsten for $J / \psi$. Boxes around points represent systematic error. . . . . . . . . . . . . . . . . . . . . . 122

4.4 $R_{p A}$ vs $x_{F}$ for carbon, iron and tungsten for DY. Boxes around points represent systematic error. . . . . . . . . . . . . . . . . . . . . . . . . . . . . . 123

4.5 $R_{p A}$ vs $p_{T}(\mathrm{GeV} / \mathrm{c})$ for carbon, iron and tungsten for both $J / \psi$ and DY. Boxes around points represent systematic error. . . . . . . . . . . . . . . . . . . . . 124

4.6 $R_{p A}$ vs $x_{F}$ for carbon, iron and tungsten for both $J / \psi$ and DY. Boxes around points represent systematic error. . . . . . . . . . . . . . . . . . . 125

4.7 $J / \psi R_{p A}$ vs $p_{T}(\mathrm{GeV} / \mathrm{c})$ for iron and tungsten as measured in E906 and E866. Liquid deuterium $\left(L D_{2}\right)$ was the base target for E906 ratio measurements, while Beryllium (Be) was the base target for E866 ratio measurements. Boxes around points represent systematic error for E906. E866 reported a systematic error of 2 $\%$ across all $p_{T}$ bins [25] . . . . . . . . . . . . . . . . . . 126 
4.8 DY $R_{p A}$ vs $p_{T}(\mathrm{GeV} / \mathrm{c})$ for iron and tungsten as measured in E906 and E866. Liquid deuterium $\left(L D_{2}\right)$ was the base target for E906 ratio measurements, while Beryllium (Be) was the base target for E866 ratio measurements. Boxes around points represent systematic error for E906. E866 reported a systematic error of $1 \%$ across all $p_{T}$ bins [27]. . . . . . . . . . . . . . . . . . . 127

$4.9 \mathrm{~J} / \psi R_{p A}$ vs $x_{F}$ for iron and tungsten as measured in E906 and E866. Liquid deuterium $\left(L D_{2}\right)$ was the base target for E906 ratio measurements, while Beryllium (Be) was the base target for E866 ratio measurements. Boxes around points represent systematic error for E906. E866 reported a systematic error of $3 \%$ across all

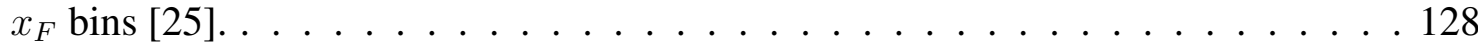

4.10 DY $R_{p A}$ vs $x_{F}$ for iron and tungsten as measured in E906 and E866. Liquid deuterium $\left(L D_{2}\right)$ was the base target for E906 ratio measurements, while Beryllium (Be) was the base target for E866 ratio measurements. Boxes around points represent systematic error for E906. E866 reported a systematic error of $1 \%$ across all $x_{F}$ bins [27]. . . . . . . . . . . . . . . . . . . . . . . 129

A.1 Intensity dependence of $J / \psi R_{p A}$ for iron for different $p_{T}(\mathrm{GeV} / \mathrm{c})$ bins. $F=$ $p 0+p 1 *$ intensity $+p 2 *$ intensity ${ }^{2}$ fit was used for the extrapolation, with $p 0$ taken as the nominal $R_{p A}$ value. The fit parameters $p 1$ and $p 2$ are common for all fits.145

A.2 Intensity dependnce of $J / \psi R_{p A}$ for tungsten for different $p_{T}(\mathrm{GeV} / \mathrm{c})$ bins. $F=$ $p 0+p 1 *$ intensity $+p 2 *$ intensity ${ }^{2}$ fit was used for the extrapolation, with $p 0$ taken as the nominal $R_{p A}$ value. The fit parameters $p 1$ and $p 2$ are common for all fits. 146

A.3 Intensity dependence of DY $R_{p A}$ for iron for different $p_{T}(\mathrm{GeV} / \mathrm{c})$ bins. $F=$ $p 0+p 1 *$ intensity $+p 2 *$ intensity $^{2}$ fit was used for the extrapolation, with $p 0$ taken as the nominal $R_{p A}$ value. The fit parameters $p 1$ and $p 2$ are common for all fits. 147

A.4 Intensity dependence of DY $R_{p A}$ for tungsten for different $p_{T}(\mathrm{GeV} / \mathrm{c})$ bins. $F=$ $p 0+p 1 *$ intensity $+p 2 *$ intensity $^{2}$ fit was used for the extrapolation, with $p 0$ taken as the nominal $R_{p A}$ value. The fit parameters $p 1$ and $p 2$ are common for all fits. 148

A.5 Intensity dependence of $J / \psi R_{p A}$ for iron for different $x_{F}$ bins. $F=p 0+p 1 *$ intensity $+p 2 *$ intensity ${ }^{2}$ fit was used for the extrapolation, with $p 0$ taken as the nominal $R_{p A}$ value. The fit parameters $p 1$ and $p 2$ are common for all fits. . . . . . . 149

A.6 Intensity dependence of $J / \psi R_{p A}$ for tungsten for different $x_{F}$ bins. $F=p 0+p 1 *$ intensity $+p 2 *$ intensity ${ }^{2}$ fit was used for the extrapolation, with $p 0$ taken as the nominal $R_{p A}$ value. The fit parameters $p 1$ and $p 2$ are common for all fits. . . . . . 150 
A.7 Intensity dependence of DY $R_{p A}$ for iron for different $x_{F}$ bins. $F=p 0+p 1 *$ intensity $+p 2 *$ intensity ${ }^{2}$ fit was used for the extrapolation, with $p 0$ taken as the nominal $R_{p A}$ value. The fit parameters $p 1$ and $p 2$ are common for all fits. . . . . 151

A.8 Intensity dependence of DY $R_{p A}$ for tungsten for different $x_{F}$ bins. $F=p 0+p 1 *$ intensity $+p 2 *$ intensity ${ }^{2}$ fit was used for the extrapolation, with $p 0$ taken as the nominal $R_{p A}$ value. The fit parameters $p 1$ and $p 2$ are common for all fits. . . . . . 152 


\begin{abstract}
A measurement of the suppression of $J / \psi$ meson production in heavy ion interactions relative to their production in $\mathrm{p}+\mathrm{p}$ interactions has been suggested to be an important probe in identifying the possible phase transition of hot nuclear matter to a quark-gluon plasma (QGP). A similar suppression effect has also been observed in cold nuclear matter (CNM) involving heavy nuclei, not due to QGP formation. Possible cold nuclear matter effects range from nuclear absorption, to parton energy loss, to modifications of nuclear parton distribution functions (nPDFs). This suppression and other signatures of CNM effects exhibit strong kinematic dependences with the Feynman-x $\left(x_{F}\right)$ and transverse momentum $\left(p_{T}\right)$ of the produced vector meson. In order to establish a baseline for different contribu-tions to the nuclear modification of $J / \psi$ production, direct comparison to the Drell-Yan process of quark-antiquark annihilation to dileptons, with little to no final state effects, provides very valuable information. We perform measurements seeking to obtain a better quantitative understanding of these nuclear effects in the E906/SeaQuest experiment at Fermi National Accelerator Laboratory, a fixed-target experiment that measures $J / \psi, \psi$ ' and Drell-Yan (DY) signals from a $120-\mathrm{GeV}$ proton beam colliding with protons and different nuclear targets. In this dissertation, we present measurements of the nuclear mod-ification of $J / \psi$ meson production and Drell-Yan pair production as a function of $x_{F}$ and $p_{T}$ at SeaQuest and we compare results from the experiment's predecessor, E866/NuSea. Similar nuclear modification is observed for DY production while an additional suppression for $J / \psi$ meson production is observed in SeaQuest. These results shed light on the phenomenology of partons and partonic bound states in a variety of nuclear media.
\end{abstract}




\section{Chapter 1 Introduction}

The motivation for nuclear dependence studies at SeaQuest will be presented in this chapter, along with an overview of the physics at play. It begins with a discussion on the Standard Model of particle physics and the fundamental theory guiding all related quark and gluonic interactions in hadrons, Quantum Chromodynamics (QCD), along with the tecniques used to explore these QCD systems. Theoretical and experimental background on production mechanisms and nuclear dependences observed for both Drell-Yan and $J / \psi$ processes will also be examined. Finally, the kinematic setup and specific advantages of performing these nuclear dependence measurements at SeaQuest, in particular those related to the Feynman-x $\left(x_{F}\right)$ and transverse momentum $\left(p_{T}\right)$ of the produced muon pair in each process, will be covered in this section.

\subsection{The Standard Model}

Since the 1930s, the theoretical and experimental efforts of many physicists have allowed us to understand the basic structure of matter in a surprisingly succinct way thus far: a few elementary particles governed by four fundamental forces comprise all of the observable universe. The Standard Model (SM) of particle physics, developed in the 1970s, summarizes our best understanding of these particles and their mediating forces. Although there appear to be some deficiencies and a need for various extensions to the SM theory, it has become a well-verified physics theory predicting and confirming a vast array of phenomena, mechanisms and results [1].

The SM theory has identified quarks, leptons, force mediating gauge bosons and a Higgs boson as the building block of the visible universe. Figure 1.1 shows a schematic overview of these, 
including different intrinsic properties of these particles like electric charge, spin, mass and color charge. There are six types of quarks referred to as flavors, up $(u)$, down $(d)$, charm $(c)$, strange $(s)$, top $(t)$ and bottom $(b)$, each with their respective anti-particles. Leptons represent the second set of six fundamental particles in the SM, the electron $(e)$, the muon $(\mu)$, tau $(\tau)$, and their neutrino counterpart, each also with their respective anti-particles. The force mediators between quarks and leptons are the gauge bosons, where the force carrier of the electromagnetic force is the photon $(\gamma)$, of the strong nuclear force is the gluon, of the weak force are the $W^{ \pm}$and $Z^{0}$, and the graviton, which has not been verified experimentally, is theorized to be the carrier of gravitational force (it should be noted that Gravity is not a part of the SM). Lastly, the Higgs boson, discovered at the LHC in 2012, is a particle associated to the Higgs field and in turn the mechanism thought to give mass to elementary particles. 


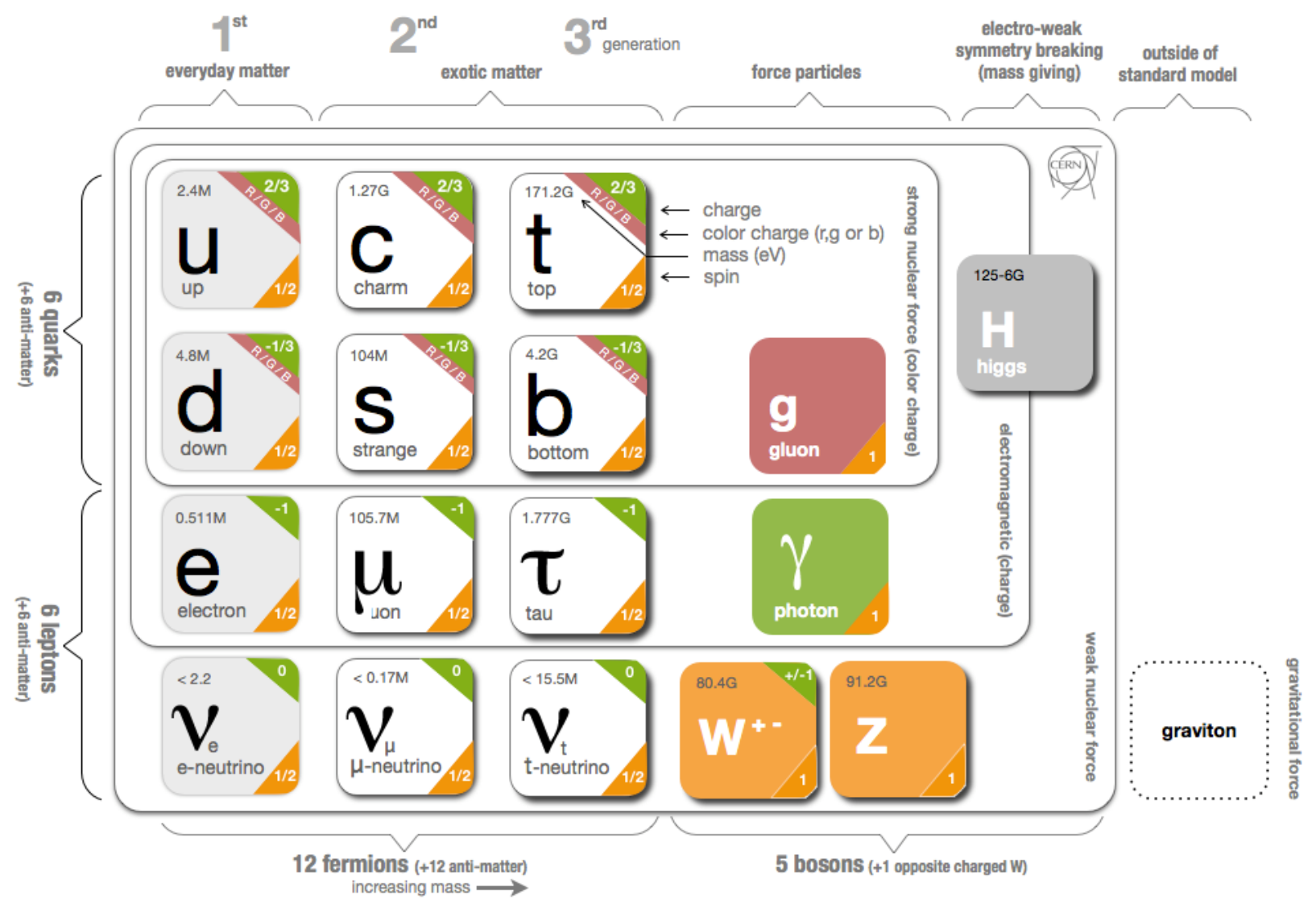

Figure 1.1: The Standard Model of particle physics [1].

\subsection{Quantum Chromodynamics}

The strong nuclear force binds quarks together via the exchange of gluons. The gauge theory that describes the strong interaction between quarks and gluons (partons) is Quantum Chromodynamics $(\mathrm{QCD})$. It is the $\mathrm{SU}(3)_{\text {color }} \mathrm{SM}$ component of $\mathrm{SU}(3)_{\text {color }} \times \mathrm{SU}(2)_{\text {weak }} \times \mathrm{U}(1)_{\gamma}$ [2]. Analogous to electric charge, partons carry color charges (red, green and blue), along with their respective anticolors. A combination of three colors, three anti-colors or a color and the same anti-color can produce baryons, like the proton and neutron, made up of three quarks, and mesons, like the $J / \psi$ and the pion, comprised of a quark-antiquark pair. Unlike the force mediator of electromagnetic (EM) interactions (the photon) which has charge neutrality, the gluon carries color and can thus 
interact with other gluons.

The interactions of partons via the strong force are controlled by the QCD Lagrangian defined as

$$
\mathcal{L}_{Q C D}=\sum_{q} \bar{\psi}_{q, a}\left(i \gamma^{\mu} \partial_{\mu} \delta_{a b}-g_{s} \gamma^{\mu} t_{a b}^{C} \mathcal{A}_{\mu}^{C}-m_{q} \delta_{a b}\right) \psi_{q, b}-\frac{1}{4} F_{\mu \nu}^{A} F^{A \mu \nu}
$$

where $\gamma^{\mu}$ is the Dirac $\gamma$-matrix, the $\psi, \bar{\psi}_{q, a}$ are the quark-field spinors for a quark of flavor $q$ and mass $m_{q}$, with a color index that sums from $a=1$ to $N_{C}=3$ ( three quark colors), $\mathcal{A}_{\mu}^{C}$ correspond to the gluon fields with $\mathrm{C}$ running from 1 to $N_{C}^{2}-1=8$ ( eight possible color anti-color gluonic combinations), $t_{a b}^{C}$ are the eight $3 \times 3$ Gell-Mann matrices of the SU(3) color group and $g_{s}$ is the QCD coupling constant.

The QCD Lagrangian field tensor $F_{\mu \nu}^{A}$ is defined as:

$$
F_{\mu \nu}^{A}=\partial_{\mu} \mathcal{A}_{\nu}-\partial_{\nu} \mathcal{A}_{\mu}-g_{s} f_{A B C} \mathcal{A}_{\mu}^{B} \mathcal{A}_{\nu}^{C}
$$

where $f_{A B C}$ are the structure constants of the SU(3) group.

The QCD coupling constant is defined as $g_{s}=\sqrt{4 \pi \alpha_{s}}$, where $\alpha_{s}$ is given by

$$
\alpha_{s}=\frac{g^{2}(\mu)}{4 \pi}=\frac{12 \pi}{\left(33 N_{C}-2 n_{f}\right) \ln \left(\frac{\mu^{2}}{\Lambda_{Q C D}^{2}}\right)}
$$

where $N_{C}$ is the number of colors, $n_{f}$ is the number of flavors of quarks in the summation, $\mu$ is the renormalization scale, $\Lambda_{Q C D}$ is the QCD scale $(\sim 200 \mathrm{MeV})[3]$.

The strength of interaction between particles engaging via a particular fundamental force is directly correlated to its coupling constant. Figure 1.2 compares the dependence of QED and QCD coupling constants on $Q^{2}$, the momentum transfer between two particles (the resolution of the probe). As can be seen in 1.2, the $\alpha_{Q E D}$ trends towards infinity with increasing $Q^{2}$ values, a direct consequence of the EM force becoming stronger due to the absence of screening from the surrounding vacuum as the EM probe approaches a charge. Conversely, the strong force coupling constant experiences a logarithmic decrease with increasing $Q^{2}$, which is a direct consequence of 


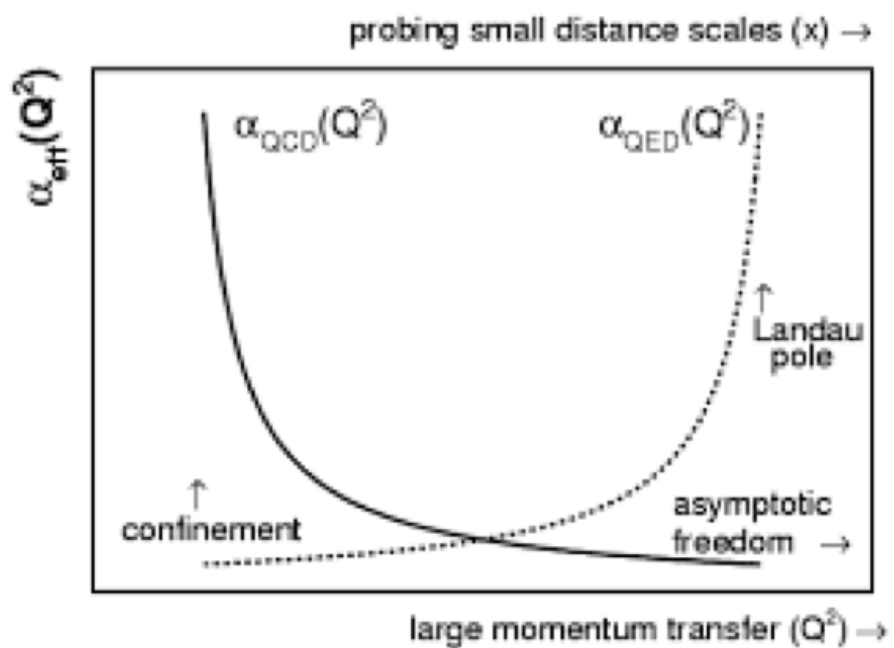

Figure 1.2: The dependence of QED and QCD coupling constants to $Q^{2}[4]$

the charge screening that takes place when a "colored" probe approaches another colored charge, thereby minimizing the strength of the force. The fact that $\alpha_{Q C D}$ becomes small in the highenergy, short-distance regime is the distinctive feature of QCD referred to as asymptotic freedom, i.e. objects with color feel almost no force when close together $[5,6]$. On the other hand, as the separation between colored charges increases, the coupling constant trends towards infinity, making it impossible to observe them in isolation, a principle known as confinement.

Asymptotic freedom allows a perturbative treatment of systems in a regime where this phenomena is expected to occur (small $\alpha_{Q C D}$ ). One can thus perform perturbative expansions to a designated order with small parameters of $\alpha_{Q C D}$ in order to evaluate important physical quantities. The confinement experienced by colored species complicates perturbative QCD (pQCD) calculations, which are normally carried out in terms of individual quark and gluon fields, in regimes with large $\alpha_{Q C D}$. This non-perturbative, long-distance behavior of QCD requires other predictive techniques to achieve a complete picture of the QCD system in question.

Factorization theorems provide a way to constrain the non-perturbative nature of QCD systems phenomenologically, while still being able to rely on pQCD calculations for the characterization of short-range interactions. A systematic separation of the hard, short-distance component of a QCD interaction from the soft, long-distance part is possible due to the absence of interference between 
these two types of dynamics. [7]. The factorized non-perturbative part can be examined via established QCD operator expressions which are themselves defined by functions with physical significance like parton distribution functions (PDFs) or fragmentation functions (FFs). PDFs represent the number densities (can be thought of as probability densities at leading order) of finding partons in the hadrons and characterize the long-distance physics of the initial states. FFs re, present the probability densities of producing hadrons from partons and characterize final state dynamics. Both of these sets of functions are understood to be "universal" (not process-dependent) and they satisfy renormalization group equations that predict and dictate their scale dependence.One can exploit this theorem by experimentally evaluating PDFs and/or FFs in a particular kinematic range and with the use evolution equations, extrapolate to other regimes. The same can be done between physics processes.

For a process of the form,

$$
A+B \rightarrow \mu^{+}+\mu^{-}+X
$$

akin to the Drell-Yan process, where $A$ and $B$ are the interacting hadrons and $X$ represents the remnants of the interaction not including the produced muon pair, $\mu^{+} \mu^{-}$, the form of the factorization theorem, up to corrections suppressed by $Q^{2}$, is as follows:

$$
\begin{aligned}
& \frac{d^{2} \sigma}{d Q^{2} d y}=\sum_{a, b} \int_{x_{A}}^{1} \frac{d \xi_{A}}{\xi_{A}} \int_{x_{B}}^{1} \frac{d \xi_{B}}{\xi_{B}} f_{a / A}\left(\xi_{A}, Q^{2}, M^{2}\right) H_{a b}\left(x_{A} / \xi_{A}, x_{B} / \xi_{B}, Q^{2}\right) \\
& \times f_{b / B}\left(\xi_{B}, Q^{2}, M^{2}\right)
\end{aligned}
$$

where $Q^{2}$ is the square of the muon pair mass, $y$ is the rapidity of the muon pair, $H_{a b}$ is the hard scattering cross-section derived perturbatively and $f_{a / A}$ and $f_{b / B}$ include all the relevant parton distributions to the process [7]. For specific definitions see Section 1.5. Factorization theorem applications are employed at SeaQuest and will be discussed in more detail later in this section. 


\subsection{The Drell-Yan process}

The internal structure of nucleons determines their fundamental properties and in turn the properties of nuclei, making a primary field of study within nuclear physics. One of the ways that nucleon structure can be examined is via the scattering of leptons, typically electrons or muons. EM probes are a preferred tool in many of these experimental endeavors given that 1) QED is a well-understood interaction and 2) leptons are not composite probes, providing a systematic calculation schema for result evaluations. Moreover, with the use of well-defined monoenergetic beams, electrons and muons can be easily accelerated and standard particle detection techniques and hardware can provide extremely accurate measurements of these particles.

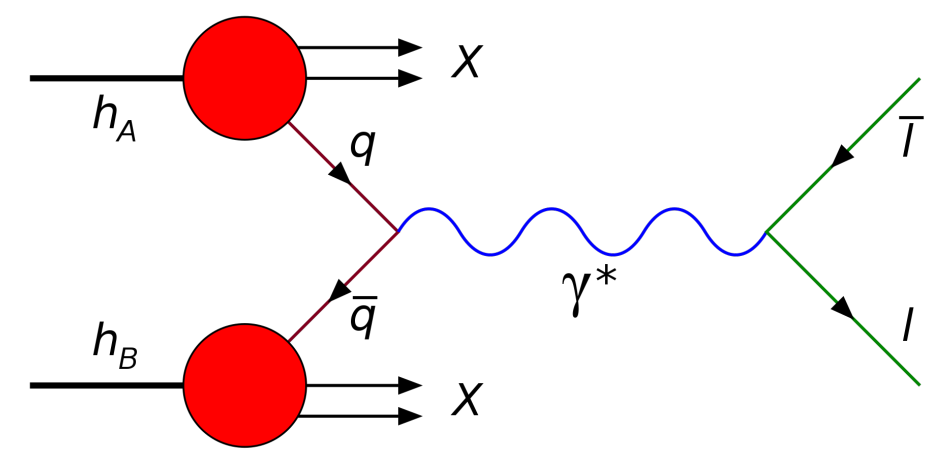

Figure 1.3: Diagram of DY process

The most relevant scattering or production process for the scope of this thesis is the Drell-Yan (DY) process, where a quark of one hadron and an antiquark of another hadron annihilate into a virtual photon. The virtual photon decays into a pair of oppositely-charged leptons. This process was postulated by Sidney Drell and Tung-Mow Yan in 1970 but was first observed by J.H. Christenson et al., where a production of massive lepton pairs in $29 \mathrm{GeV} / \mathrm{c}$ proton Uranium highenergy collisions were measured [9, 8]. In Figure 1.4, two notable features can be observed: 1) A shoulder near an invariant mass of $\sim 3.1 \mathrm{GeV}$, now understood to be the $J / \psi$ particle and its resonance states. 2) A sharply decreasing cross-section at higher invariant mass. These experimental signatures will be useful in the isolation of different process signals at SeaQuest. 


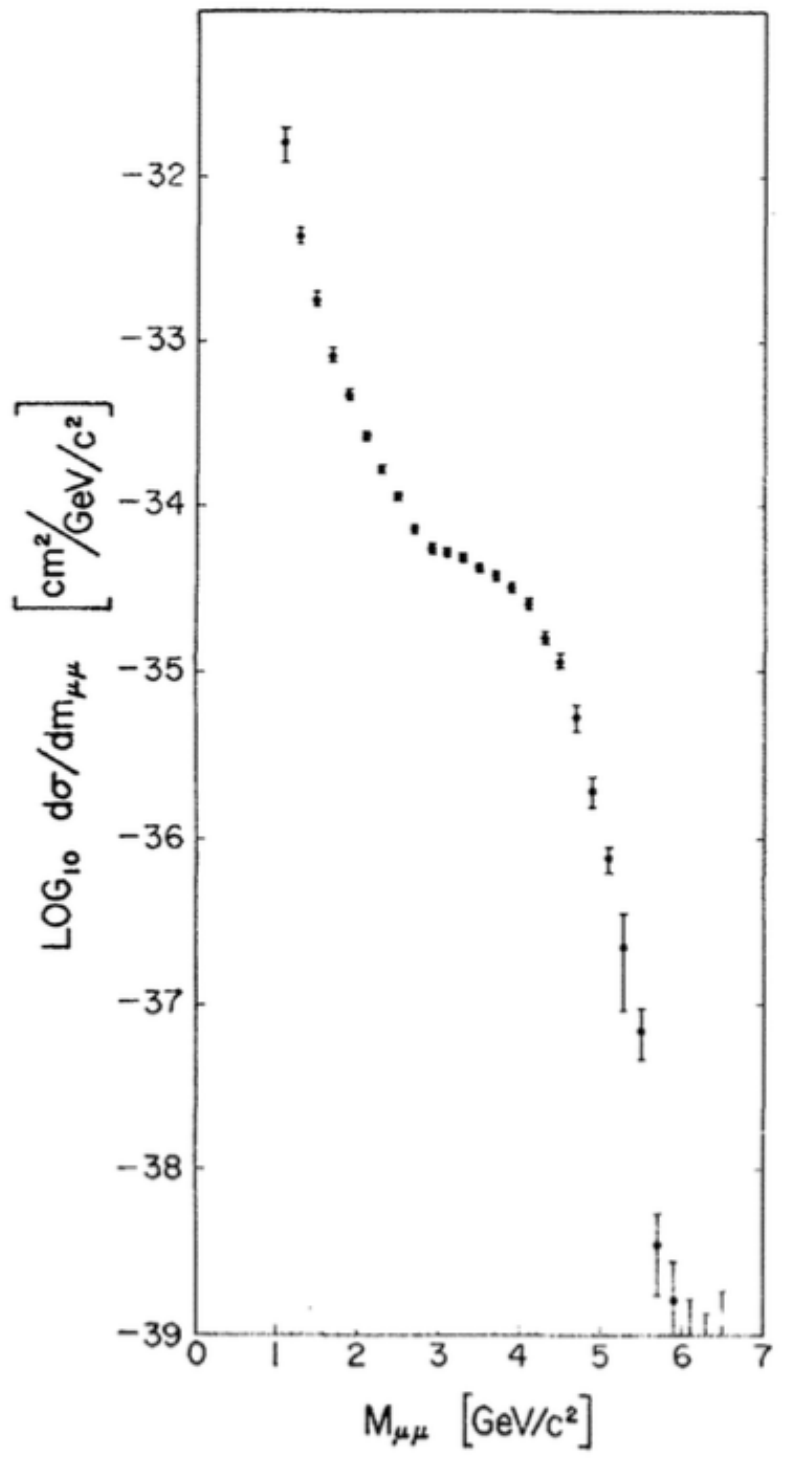

Figure 1.4: DY cross-section as a function of the invariant mass of muon pairs [8] 


\section{$1.4 J / \psi$ production}

The $J / \psi$ particle is a flavor-neutral meson comprised of a charm and anti-charm quark and it is the most common form of charmonium (charm anti-charm bound states) given its low rest mass. Its discovery was made independently by a research group at the Stanford Linear Accelerator Center and one at Brookhaven National Laboratory in 1974 [10]. Charmonia come from the quarkantiquark annihi-lation and gluon-gluon fusion partonic-level processes. The charmonia production diagrams from quark-antiquark annihilation and gluon-gluon fusion are shown in Figure 1.5.

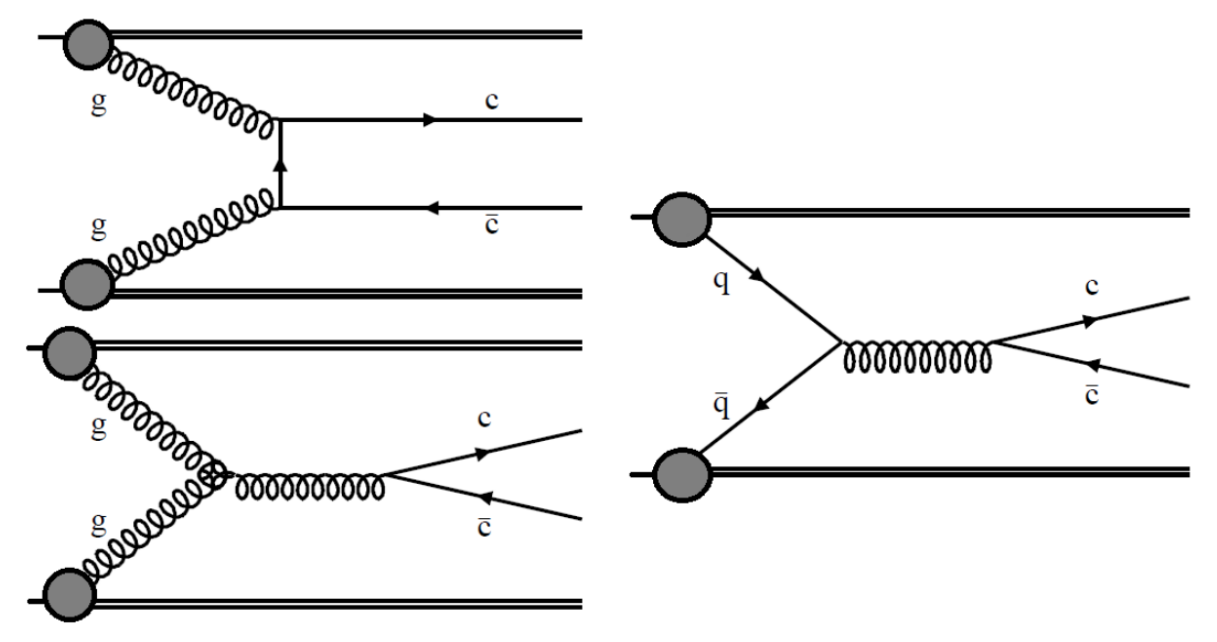

Figure 1.5: Partonic diagrams of $J / \psi$ production

The $J / \psi$ yield is proportional to the convolution of hard partonic $2 \rightarrow 2$ cross-sections and the parton density of $q(x), \bar{q}(x)$ and $g(x)$ discussed in more detail in Section 1.5. Here, $x$, is the fraction of the proton momentum carried by a struck parton, later defined within the scope of SeaQuest as either $x_{\text {target }}$ or $x_{\text {beam }}$ depending on whether the parton came from the beam or the target $[11,12]$.

The relative size or contribution to the total $c \bar{c}$ cross-section from each process is dependent on both center-of-mass energy, $\sqrt{s}$, and x-Feynman, $x_{F}$. Generally, at lower $x_{F}$, gluon fusion is the dominant process leading to $c \bar{c}$ production but at higher $x_{F}, q \bar{q}$ annihilation begins to play a leading role, in some cases becoming the primary production channel. The critical point where 

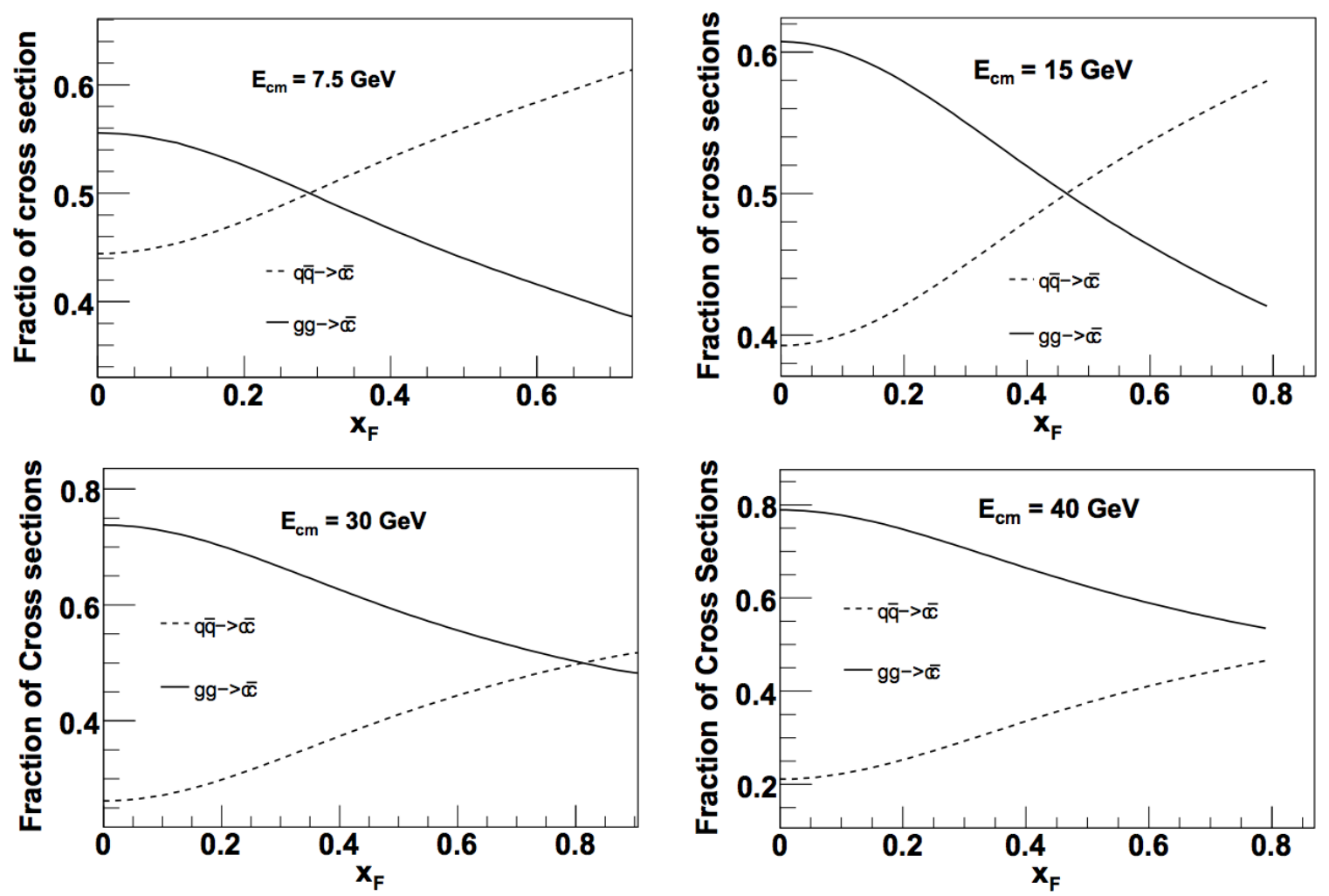

Figure 1.6: Ratio of quark annihilation and gluon fusion cross-sections for $c \bar{c}$ production in p-p collisions to their sum vs. $x_{F}$ at varying $E_{c m}=\sqrt{s} . E_{c m}$ at SeaQuest is $15.4 \mathrm{GeV}$. [11].

quark annihilation overtakes gluon fusion is not fixed given the fact that it is largely dependent on the collision energies at play. Overall, the higher the $\sqrt{s}$, the greater the gluonic contributions to the cross-section, as seen in Figure 1.6. However, at lower energies, $q \bar{q}$ channel merits important consideration in the characterization of $c \bar{c}$ creation. The $\sqrt{s}$ at SeaQuest is $15.4 \mathrm{GeV}$, suggesting dominance of the $q \bar{q}$ channel above $x_{F} \sim 0.45$, as seen in Figure 1.6. Moreover, one can examine parton distributions at a $Q^{2}=M_{J / \psi}{ }^{2}$. A beam parton at large-x is needed to reach large values of $x_{F}$, typically also corresponding to a small $\sqrt{s}$ (see Eqs. 1.7 and 1.10). Figure 1.7 shows the flavor-separated parton distributions at $Q^{2}=M_{J / \psi^{2}}$. Noting that the gluon distribution is scaled down by a factor of ten, one must go to $\mathrm{x}$ values greater than approximately 0.2 for the quark distributions to dominate, leading to the dominance of the $q \bar{q}$ production channel for $J / \Psi$ only at large $x_{F}$ values. The degree to which it matches or overcomes gluonic contributions and at what 


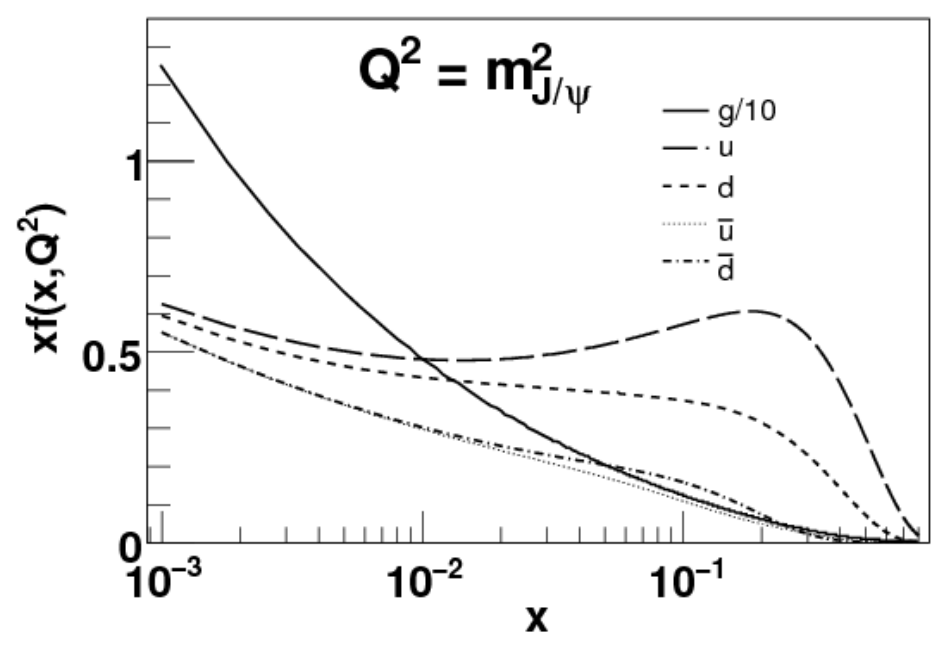

Figure 1.7: Parton distributions as a function of $\mathrm{x}$ with $Q^{2}=M_{J / \psi}{ }^{2}[11]$.

$x_{F}$ value is ultimately mediated by the underlying collision energy [11].

Figures 1.8 and 1.9 show differential cross-sections as a function of $x_{F}$ for beam energies akin to those used in SeaQuest and E866, respectively. A comparison of the top-right panel of Figure 1.6 to Figure 1.8 shows that they appear to disagree regarding the $x_{F}$ value at which there are equal leading-order contributions from the $q \bar{q}$ and $g g$ channels, with Figure 1.6 indicating a value of $x_{F} \sim 0.45$ and Figure 1.8 indicating a value of $x_{F} \sim 0.2$. Without any uncertainties provided by the authors of these plots, no conclusive statement can be made on their consistency. However, it is clear that the $q \bar{q}$ channel dominates for $x_{F} \gg 0.1$ at SeaQuest's center-of-mass energy. Given the particular kinematic coverage of each experiment, it can be expected that $J / \psi$ production at SeaQuest is sensitive to quark and anti-quark as well as gluon distributions, while E866 primarily had sensitivity to gluon distributions. 


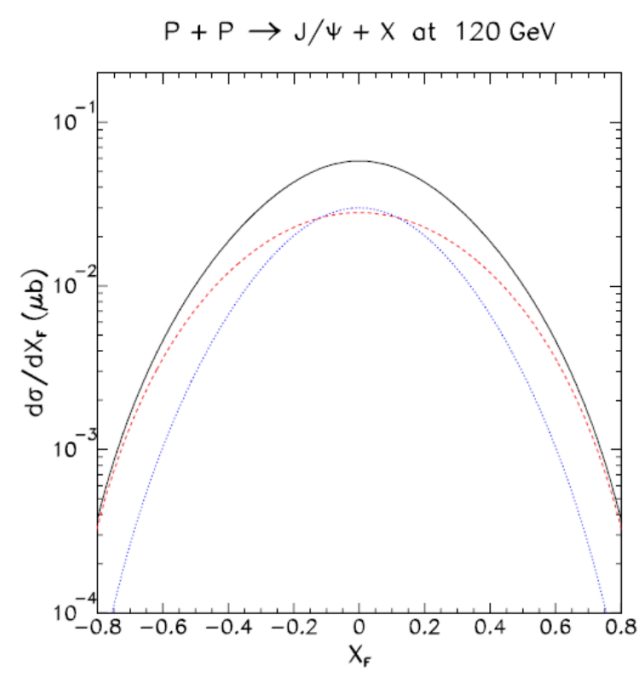

Figure 1.8: Differential cross-sections as a function of $x_{F}$ at $120 \mathrm{GeV}$ beam energy. The red curve relates to the contribution from $q \bar{q}$ annihilation and the blue curve from gluon-gluon fusion [13].

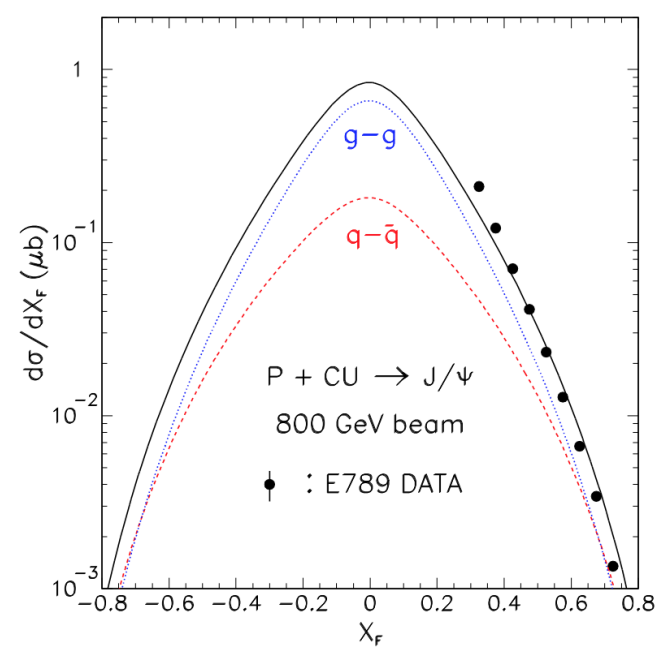

Figure 1.9: Differential cross-sections as a function of $x_{F}$ at $800 \mathrm{GeV}$ beam energy. The red curve relates to the contribution from $q \bar{q}$ annihilation and the blue curve from gluon-gluon fusion [14, 13]. 


\subsection{Process kinematics at SeaQuest}

The kinematic properties of the generated dimuons via the DY process correspond to the properties of their propagator, i.e. the virtual photon $\left(\gamma^{*}\right)$ that decayed from quark anti-quark annihilation. Once the tracks of these dimuons are reconstructed and momenta extracted, the measured 4-momentum of the muons in the lab frame are used to determine the 4-momentum of the virtual photon in the lab frame, from which one can get the mass of the dimuon. Once this 4-momentum is boosted onto the Collins-Soper frame, one can obtain $x_{F}$ which together with the mass, can be used to derive $x_{\text {beam }}$ and $x_{\text {target }}$. A more thorough exposition of this process follows below:

From the known beam energy of $120 \mathrm{GeV}$, one can calculate the center-of-mass energy, $\sqrt{s}$, for a proton (p) colliding with a proton at rest (fixed-target experiment) as follows:

$$
\sqrt{s}=\sqrt{2 E_{\text {beam }} m_{p}}
$$

Now, $s$ and the dimuon momenta can be used to calculate different variables of the virtual photon including: longitudinal momentum $p_{l}$, invariant mass of the virtual photon $M_{\gamma^{*}}$, energy of the virtual photon $E, \tau$, rapidity $y$ and $x_{F}$. This is done as follows:

$$
\begin{gathered}
\tau=M_{\gamma^{*}}^{2} / s=x_{\text {beam }} x_{\text {target }} \\
y=\frac{1}{2} \ln \left(\frac{E+p_{l}}{E-p_{l}}\right) \\
x_{\text {beam }}=\frac{P_{\text {target }} * P_{\text {sum }}}{P_{\text {target }} * P_{\text {cms }}} \\
x_{\text {target }}=\frac{P_{\text {beam }} * P_{\text {sum }}}{P_{\text {beam }} * P_{\text {cms }}}
\end{gathered}
$$

where $P_{\text {beam }}=\left(0,0, \sqrt{E_{\text {beam }}^{2}-m_{p}^{2}}, E_{\text {beam }}\right), P_{\text {target }}=\left(0,0,0, m_{p}\right), P_{c m s}=P_{\text {beam }}+P_{\text {target }}$ and 


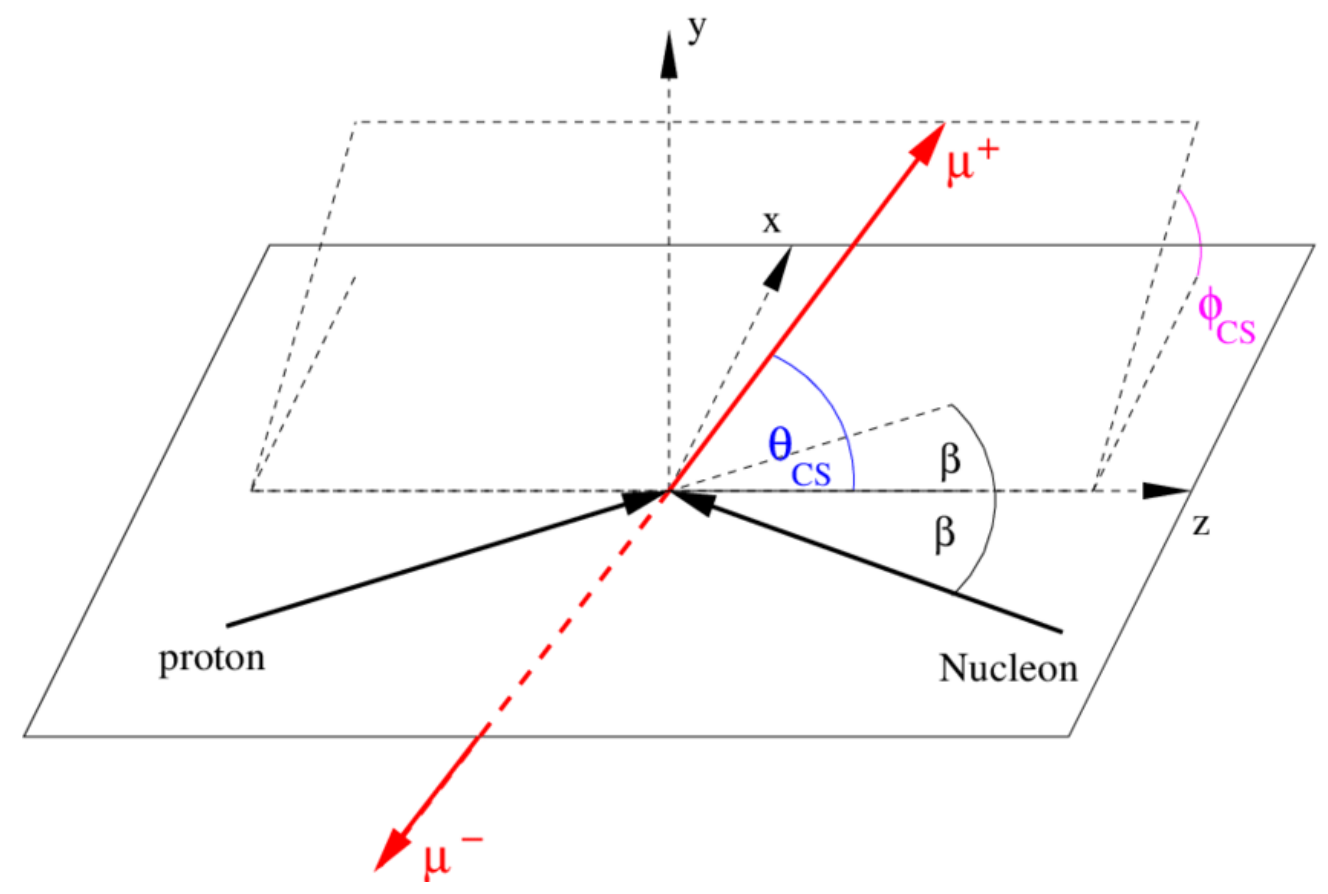

Figure 1.10: Collins-Soper frame is the center of mass frame of the dileptons produced in hadronhadron collisions. $\theta_{C S}$ is the polar angle and $\phi_{C S}$ is the azimuthal angle [15].

$P_{\text {sum }}=P_{\text {pos }}+P_{\text {neg }}$ where $P_{\text {pos }}$ and $P_{\text {neg }}$ are the four vectors of the $\mu^{+}$and $\mu^{-}$respectively.

$$
\begin{array}{r}
x_{F}=\frac{p_{l}}{p_{l}^{\text {max }}} \approx x_{\text {beam }}-x_{\text {target }} \\
p_{l}^{\text {max }}=\frac{\sqrt{s}}{2}\left(1-\frac{m_{\gamma^{*}}^{2}}{s}\right)
\end{array}
$$

It should be noted that the $p_{T}, \theta$ and $\phi$ of the virtual photon can also be evaluated from the momenta of the dileptons. The geometric schema of some of these variables can be seen in Figure 1.10. A similar kinematic analysis can be used when considering the dynamics of the $J / \psi$ meson.

The leading order cross-section for the Drell-Yan scattering cross-section is given by

$$
\frac{d^{2} \sigma}{d M^{2} d x_{F}}=\frac{4 \pi \alpha^{2}}{9 M^{4}} \frac{x_{\text {beam }} x_{\text {target }}}{x_{\text {beam }}+x_{\text {target }}} \sum_{i \in\{u, d, s, \ldots\}}^{n} e_{i}^{2}\left[f_{i}\left(x_{\text {beam }}\right) \bar{f}_{i}\left(x_{\text {target }}\right)+\bar{f}_{i}\left(x_{\text {beam }}\right) f_{i}\left(x_{\text {target }}\right)\right]
$$

where $f_{i}\left(x_{\text {beam }}\right)$ and $\bar{f}_{i}\left(x_{\text {target }}\right)$ are the quark distributions, $x_{\text {beam }}$ and $x_{\text {target }}$ are the fractions of longitudinal momentum carried by the participating beam and target quarks, respectively, $s$ is the 
square of the center of mass energy, $\alpha$ is the fine structure constant revealing the electromagnetic nature of the process, $e_{i}$ is the quark flavor's charge and the sum is over all the quark flavors. At large values of $x$, the quark distributions are dominated by the valence regions, and at small $x$ the quark distributions are dominated by the sea.

The leading order cross-section for $J / \psi$ hadroproduction, according to the QCD factorization theorem, is defined as the convolution of the quark-antiquark annihilation and gluon-gluon fusion cross-sections:

$$
\begin{aligned}
& H_{\text {beam,target }}\left(x_{\text {target }}, x_{\text {beam }} ; M^{2}\right)=g\left(x_{\text {beam }}\right) g\left(x_{\text {target }}\right) \sigma\left(g g \rightarrow c \bar{c} ; M^{2}\right) \\
& +\sum_{i \in\{u, d, s, \ldots\}}^{n}\left[f_{i}\left(x_{\text {beam }}\right) \bar{f}_{i}\left(x_{\text {target }}\right)+\bar{f}_{i}\left(x_{\text {beam }}\right) f_{i}\left(x_{\text {target }}\right)\right] \sigma\left(q \bar{q} \rightarrow c \bar{c} ; M^{2}\right)
\end{aligned}
$$

where $g\left(x_{\text {beam }}\right)$ and $g\left(x_{\text {target }}\right)$ are the gluon distributions for the beam and target parton, respectively, $\sigma\left(g g \rightarrow c \bar{c} ; M^{2}\right)$ and $\sigma\left(q \bar{q} \rightarrow c \bar{c} ; M^{2}\right)$ are the production cross-section of the different QCD subprocesses that could generate a $c \bar{c}$ pair, $M$ here is the invariant mass of the $c \bar{c}$ [12].

The leading order differential cross-section for free $c \bar{c}$ production written in terms of $M^{2}$ and $x_{F}$ is

$$
\frac{d^{2} \sigma}{d \tau d x_{F}}=\frac{2 \tau}{\sqrt{x_{F}^{2}+4 \tau^{2}}} H_{\text {beam,target }}\left(x_{\text {target }}, x_{\text {beam }} ; x_{\text {target }} x_{\text {beam }} s\right)
$$

In order to apply this cross-section to the bound $c \bar{c}$ production, one must integrate the free production cross-section over $\tau$ from the $c \bar{c}$ production threshold to the open charm threshold. For details on this procedure see [12].

The SeaQuest spectrometer uses detectors with very forward acceptance. With this type of geometry, only dilepton pairs with high $x_{F}$ coming from a high-x beam parton $\left(x_{\text {beam }}\right)$ and a low or moderate-x target parton $\left(x_{\text {target }}\right)$ are accepted. Thus, the $f_{i}\left(x_{\text {beam }}\right) \bar{f}_{i}\left(x_{\text {target }}\right)$ term in both $J / \psi$ and DY production cross-sections dominates and the second term can dropped for future calculations using this data (see Figure 1.11). 

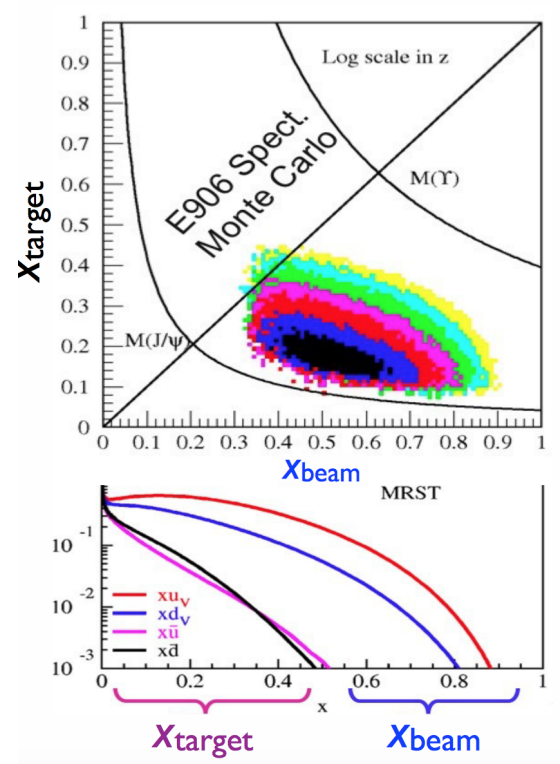

Figure 1.11: $x_{\text {beam }}$ vs. $x_{\text {target }}$ plot with simulated SeaQuest populated acceptance (top). LO parton distributions as a function of x-Bjorken (bottom) [16].

\subsection{Initial and final-state effects}

Initial and final states correspond respectively to "before" and "after" a hard collision has taken place. For the DY process, the quark and anti-quark annihilate to form a virtual photon, then become a muon pair. The dimuons have hardly any interaction with the nuclear medium, so that only initial-state effects are associated with this process. For the initial state in both processes,i.e. DY pair and $J / \psi$ production, there are a few sources that could produce and alter the $p_{T}$ associated with the subsequent lepton pair. These include, the intrinsic $p_{T}$ of initial-state partons, single or multiple elastic scattering of and gluon emission from the initial-state beam parton. Cross-section measurements of both of these processes as a function of $p_{T}$ could thus allow for the study and understanding of different initial-state signatures.

In high energy hadron-nucleus and nucleus-nucleus collisions, initial and final-state effects can change both the production rate and the momentum spectrum of the $J / \psi$ meson. The effect of the final-state interaction depends on the hadronization mechanism, i.e. how a produced heavy quark pair becomes a bound charmonium. 


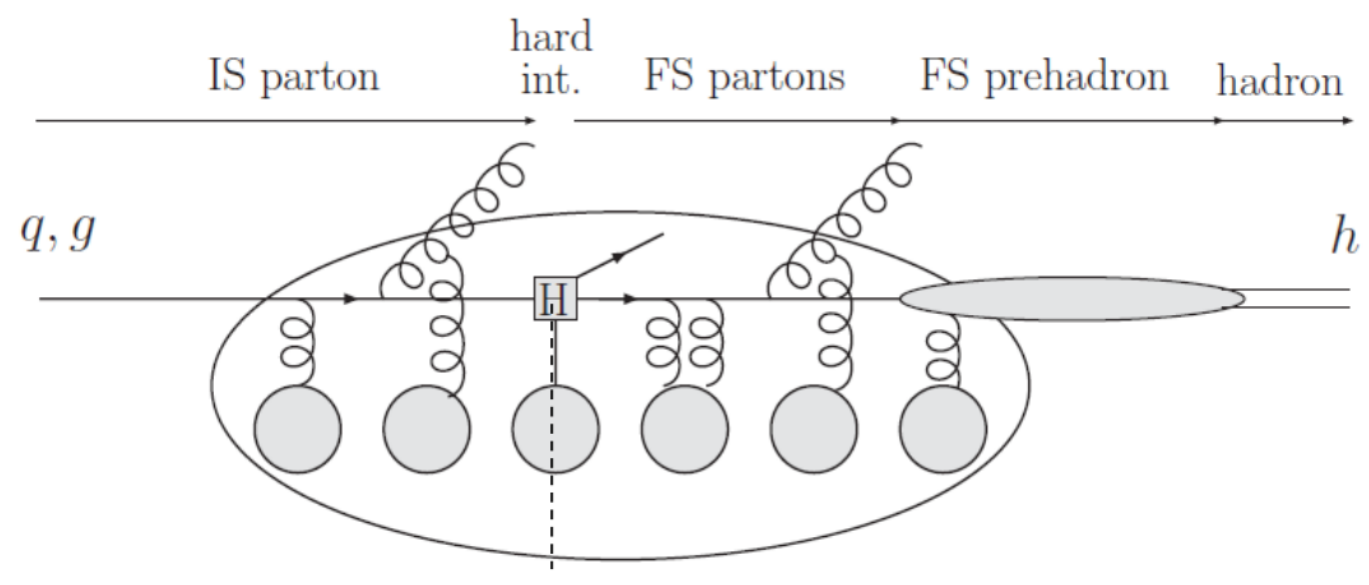

Figure 1.12: Diagram of initial and final-state effects.

After a hard collision, the virtual photon or gluon will form a $c \bar{c}$ prior to becoming a bound state. Figure 1.12 refers to this stage as the "FS prehadron" phase. The hard scattering takes place at such a short time that it is unlikely to interfere strongly with the dynamics of the nuclear medium which is effectively frozen. In contrast, the hadronization from the heavy quark pair to a bound quarkonium (final state) could be quite sensitive to the properties of the medium. In this study, the DY process is used as a controlled check for the study of final state effects since it is expected to have far less interaction with the nuclear medium, post hard collision, than the $J / \psi$ meson.

The magnitude of nuclear medium interactions can depend on whether the final state parton pair had either a color-singlet or color-octet configuration [17]. Given the g-g contribution at lower $x_{F}$ for $J / \psi$ production, different initial-state effects to that of the DY process could be expected. Studying these effects could shed light on the dynamics of partonic rescattering when a fast parton passes through nuclear matter. Moreover, the effects of the color configuration on the $c \bar{c}$ pair in the final state could be an ideal probe for exploring the non-perturbative formation mechanism in heavy quarkonium production. 


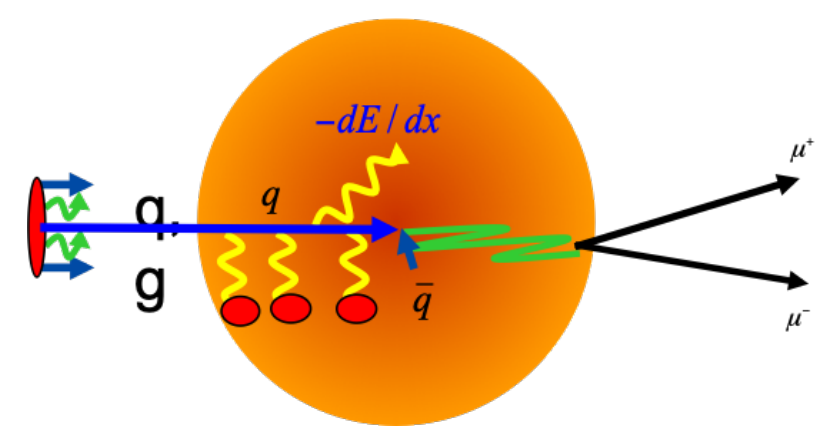

Figure 1.13: Energy loss process at SeaQuest [18].

\subsubsection{Parton energy loss}

Parton energy loss is another possible initial-state process in both $J / \psi$ and DY production studied in this thesis. As seen in Figure 1.13, as the fast parton from the projectile hadron propagates through the cold nuclear matter (i.e a heavy nucleus), it can experience energy loss before the annihilation takes place. If it does, the energy of the incoming parton immediately prior to annihilation would be different from its initial value. This modification in energy is analogous to a change in the hadron momentum fraction carried by the parton involved in the annihilation process. Thus, one would expect that a signature of the initial-state energy loss is the modification in the dimuonproduction spectrum with respect to $x$ or $x_{F}$. The magnitude of energy loss can also be expected to be greater in heavier nuclei therefore, by measuring the nuclear dependence of proton-nucleus DY or $J / \psi$ cross-sections, the energy losses can be obtained and studied. Again, the DY process is an ideal probe for the energy loss since its effect can be cleanly observed through the dimuons, given the absence of final-state effects. Fig. 1.14 shows the effect of parton energy loss on $R_{p a}$, the yield ratio of production in proton-nucleus versus proton-deuterium collisions (defined in Eq. 3.18). If there is little to no energy loss of the incoming parton, as would be expected for deuterium, then the $x_{\text {beam }}$ distributions will be centered around a nominal value. However, if the beam parton is subject to initial-state energy loss in a heavier nucleus, the $x_{\text {beam }}$ distributions in this nucleus are shifted, resulting in an overall slope for $R_{p A}$ as a function of $x_{\text {beam }}$ (or $x_{F}$ ). 


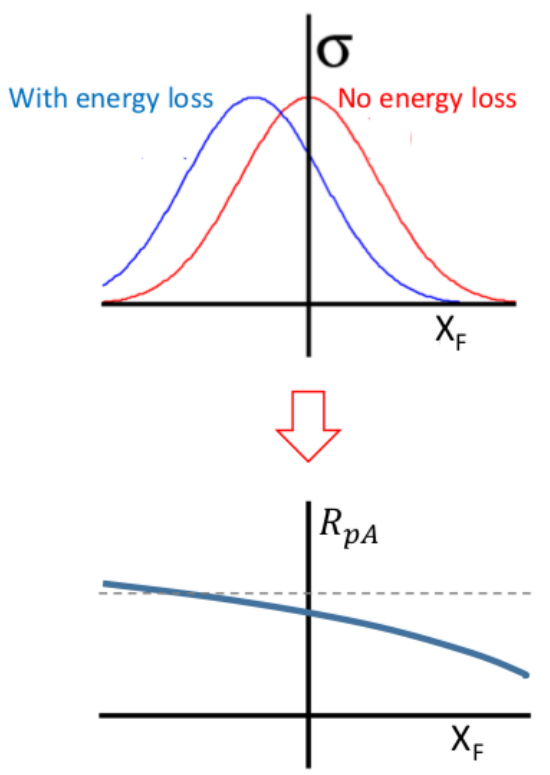

Figure 1.14: Illustration of why the yield ratio, $R_{p A}$, drops as a function of $x_{F}$ [4]. 


\subsection{2 $\mathrm{J} / \psi$ production suppression: CNM effect and QGP probe}

A suppression of $J / \psi$ production in high-energy heavy ion interactions has been suggested to be a probe in identifying the presence and properties of quark-gluon plasma (QGP) [19]. A similar final-state quarkonium production depletion observed in p-A interactions at lower energies makes it critical to understand the effects of cold nuclear matter (CNM). By doing so, we can:

- Kinematically isolate the sources of this suppression.

- Better constrain presumed QGP signatures.

- Better understand heavy quarkonium production in hadronic collisions.

Charmonium production suppression due to QGP is the result of a very particular interaction between the final-state quarks and their environment after a high-energy hard collision. Here, the confinement linear potential between the $c$ and $\bar{c}$-quark can be less than the thermal kinetic energy leading the $J / \psi$ to dissociate while their color-Coulomb interaction with partons in the plasma can screen the $c$ quark color charge from the $\bar{c}$ quark. Hadronization of the $c$ and $\bar{c}$ particles with lighter quarks can take place to form D-mesons instead of $J / \psi$ resulting in a drop [20].

CNM effects could also be causing a suppression in $J / \psi$ production (and its excited states) via various mechanisms but most notably through nuclear absorption, a final-state effect. In A-A or A-p collisions, the produced $c \bar{c}$ pairs interact with the nuclear medium before emerging. Via this interaction, the magnitude of the relative momentum of the pair increases, allowing some pairs to cross into the open charm meson sector. Each of these effects is expected to generate a reduction in the cross-section of $J / \psi$ production relative to that of p-p and they evolve differently with increasing energy density as can be seen in Figure 1.15 [22, 23].

SeaQuest has access to measure different modifications of charmonium production due to these final-state CNM effects thanks to the nucleonic diversity of the probed targets and the unique energy-momentum regimes covered. This will allow models of different CNM mechanisms to be tested and can in turn be used as input to understand what effects in high-energy heavy ion collisions are in fact due to QGP. 

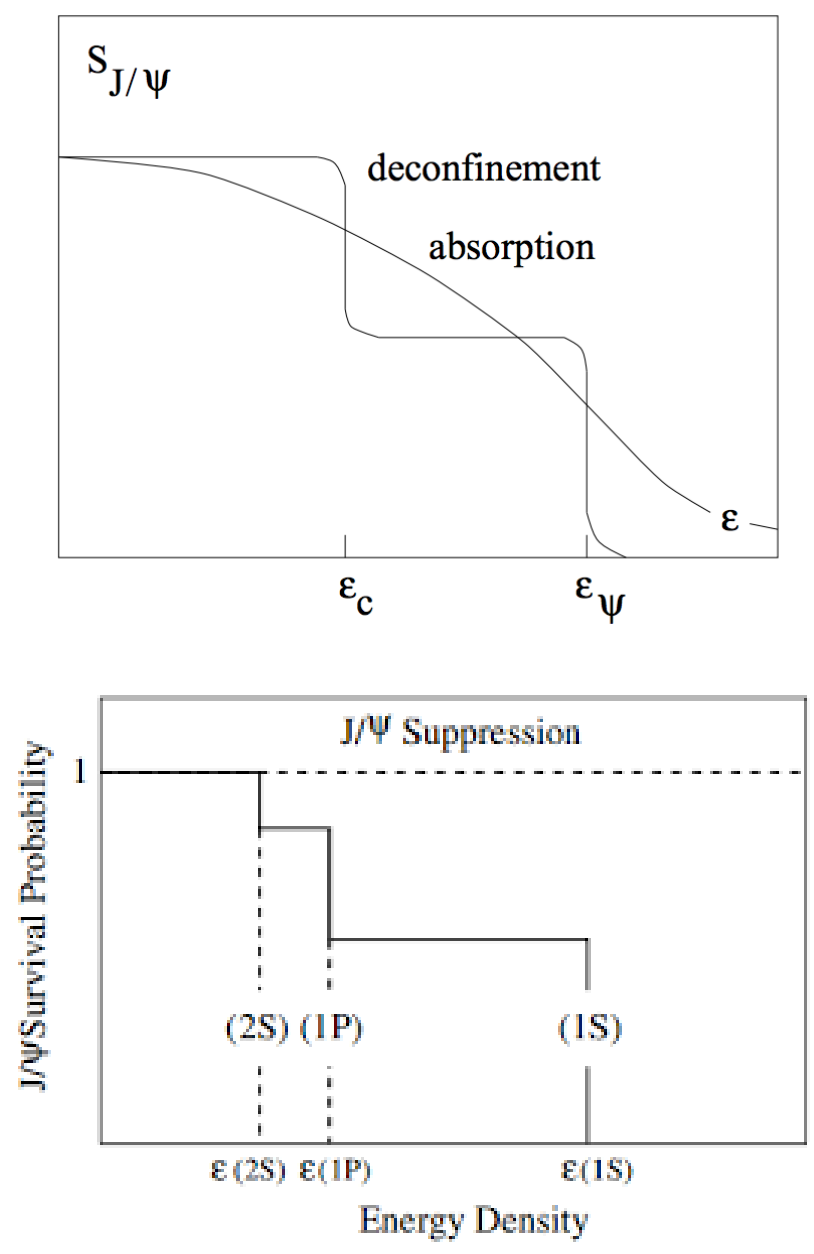

Figure 1.15: The $J / \psi$ survival probability as a function of energy density for suppression by deconfinement and by hadronic absorption (top). Sequential quarkonium suppression, where 1Sstate is $J / \psi$ (bottom) [20,21].

\subsection{Previous measurements of nuclear modification}

The effects of parton $p_{T}$-broadening and energy loss in CNM on the $p_{T}$ dependence of $J / \psi$ suppression in $\mathrm{p}-\mathrm{A}$ collisions have been studied widely among theorists and experimental collaborations. In Figures 1.16 and 1.17, model predictions were compared to data from E866 and RHIC p-A experiments at varying center-of-mass energies $(\sqrt{s})$.

These models seem to indicate that momentum broadening is responsible for the rapid variation of $J / \psi$ suppression with $p_{T}$, while medium-dependent energy loss largely affects the magnitude of $R_{p A}$. Moreover, models of $R_{p A}$ as a function of $p_{T}$ for fixed-target experiments to RHIC suggest 


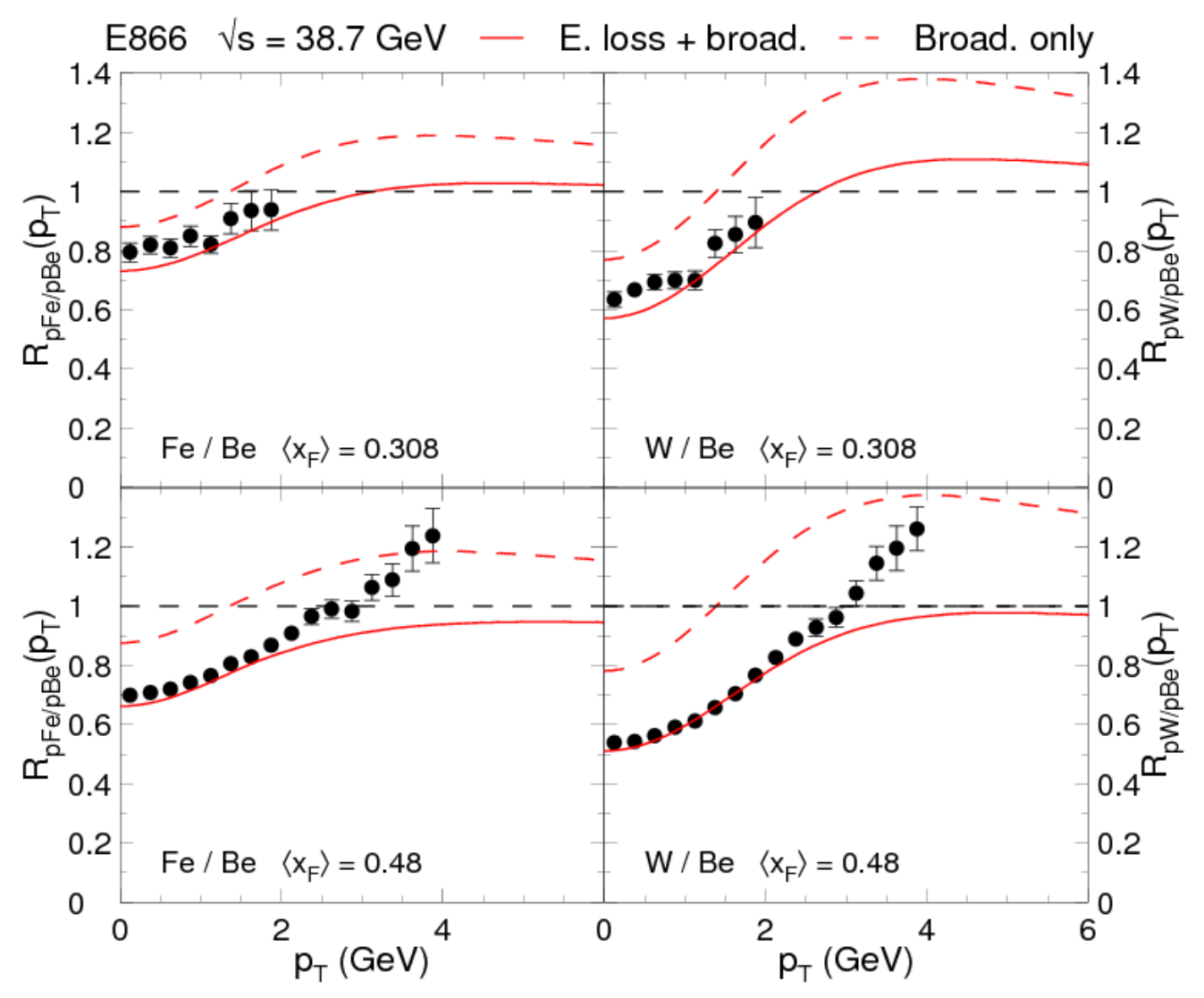

Figure 1.16: Model predictions for the $J / \psi$ nuclear suppression factor compared to the E866 data for yield ratios, $R_{F e / B e}\left(p_{T}\right)$ and $R_{W / B e}\left(p_{T}\right)$, in the intermediate and large- $x_{F}$. The dashed lines indicate the effect of momentum broadening only $[24,25]$.

that parton energy loss prompted by momentum broadening could be the dominant effect responsible for $J / \psi$ suppression in p-A collisions [24]. 

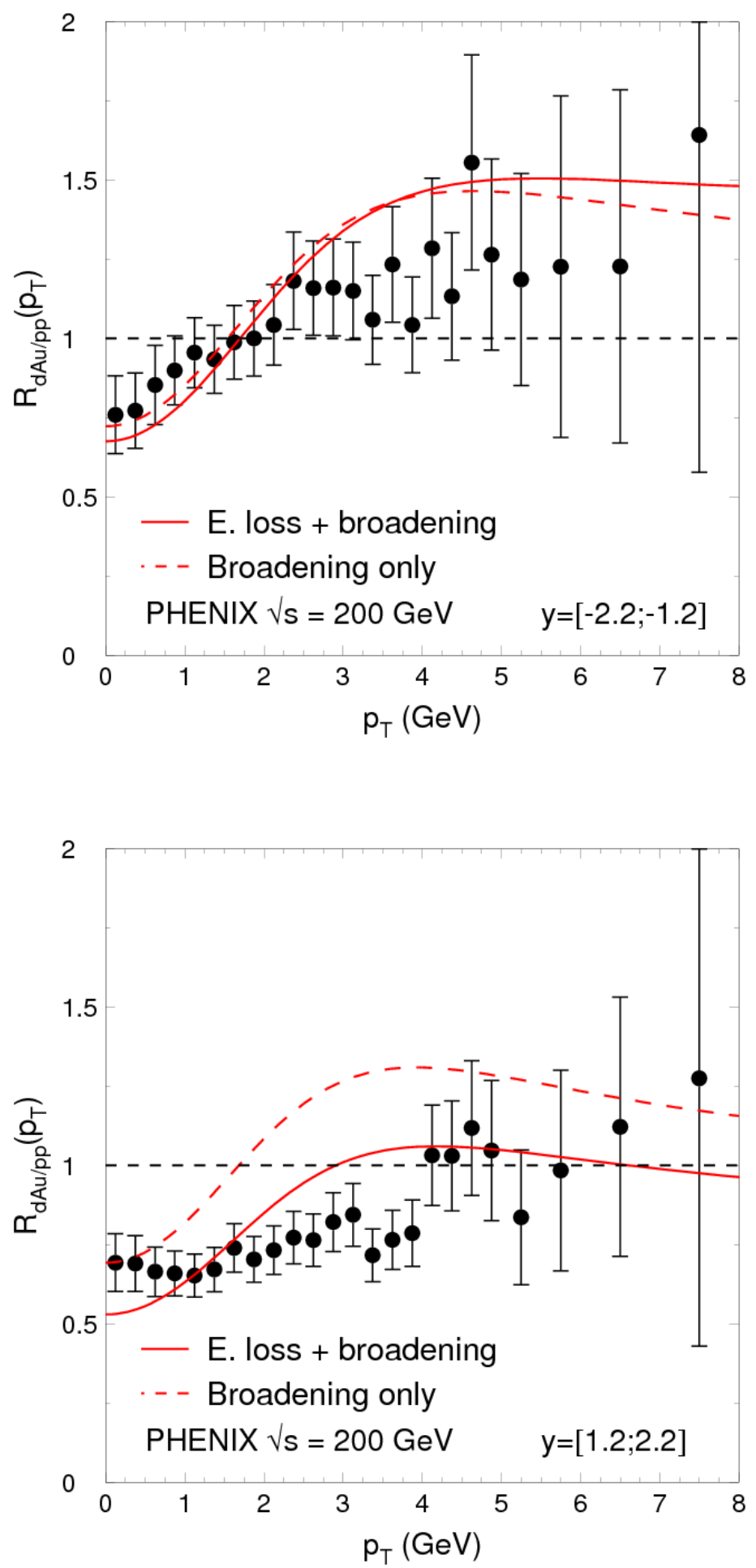

Figure 1.17: Model predictions for the $J / \psi$ nuclear suppression factor $R_{p A}\left(p_{T}\right)$ (yield ratio) in minimum bias d-Au collisions at RHIC, at backward (top) and forward (bottom) rapidities. The dashed lines indicate the effect of momentum broadening only [24, 22]. 
Fermilab experiments, E772 and E866, studied nuclear dependences of proton-induced DY production of muon pairs at a beam energy of $800 \mathrm{GeV}$, both using similar heavy solid target species to E906 (Fe and W) [26, 27]. Given that most of the E866 data came from the regime $x_{2}$ (similar to $\left.x_{\text {target }}\right)<0.05$ where one expects considerable nuclear shadowing, the EKS98 nPDF parameterization was employed to correct the shadowing effect. E866 found a very small energyloss rate consistent with no energy loss observed. [27, 18].

Even with these results, it remains an incredibly difficult task to reliably determine the parton energy loss in CNM because of the smearing of other mechanisms at play and the limited accessible experimental data. More data is needed at different kinematic regimes where other mechanisms of nuclear modification may be weaker or better constrained. SeaQuest data will push kinematic regions and expand accessible experimental data sensitive to different multivariable nuclear dependences.

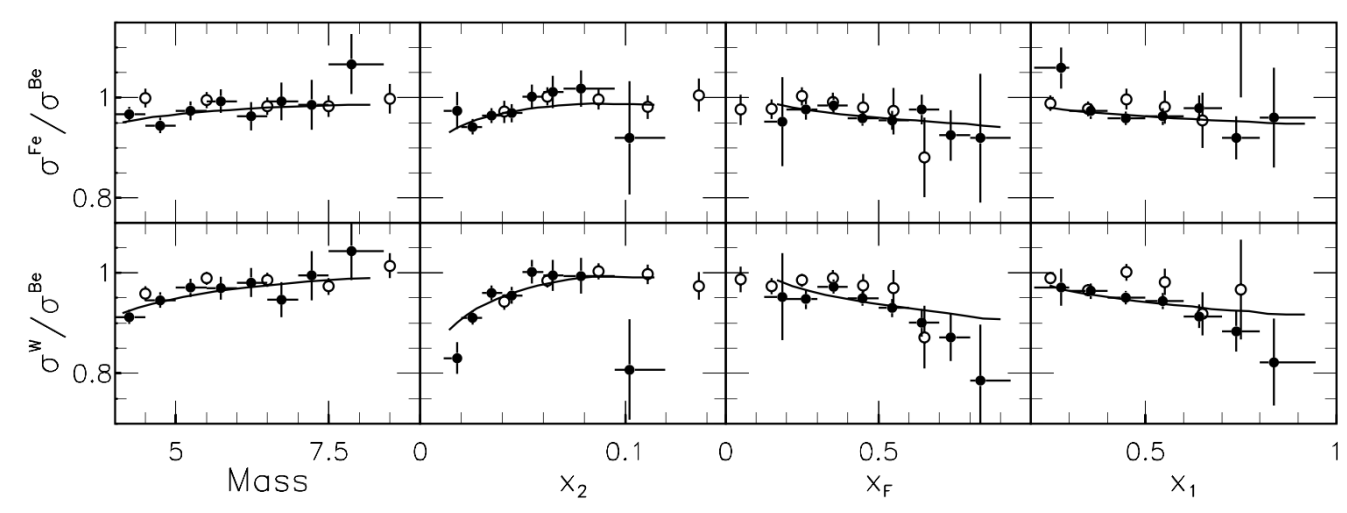

Figure 1.18: Yield ratios, $R_{\mathrm{Fe} / \mathrm{Be}}$ and $R_{W / B e}$, for DY events versus dimuon mass, $x_{2}$ (similar to $x_{\text {target }}$ ), $x_{F}$ and $x_{1}$ (similar to $x_{\text {beam }}$ ). Solid circles are results from E866 and open circles are results from its predecessor Fermilab experiment, E772. The solid curves are the predicted crosssection ratios for E866, integrated over the other variables from LO-calculations using EKS98 and MRST [26, 27]. 


\section{Chapter 2 Experimental Setup}

The E906/SeaQuest spectrometer at Fermi National Accelerator Laboratory (Fermilab) is designed to measure charged muon pairs resulting from several sources, including Drell-Yan (DY) and $\mathrm{J} / \psi$ production processes. Employing a high intensity $120-\mathrm{GeV}$ proton beam from the Main Injector at Fermilab and intercepted by several types of solid and liquid targets, the SeaQuest experiment is able to explore quark and antiquark structures in a large momentum-fraction region and measure modifications of these structures in various sized nuclei. Sharing a similar configuration to that of other Fermilab DY experiments, like E866 [28], the SeaQuest spectrometer is specifically designed to allow for result comparisons within analogous kinematic regions, while concurrently pushing the boundaries of kinematic reach not previously surveyed by these former experiments [29].

Figure 2.1 shows an illustrated graphic of the SeaQuest spectrometer. From the target "cave" to the final triggering station, the total spectrometer measures about 25 meters long. After encountering the first upstream beam-profiling hardware, discussed in Section 2.2, the beam enters the spectrometer from the left of the diagram and interacts with one target at a time for any given slow-extraction spill. The target species used by the experiment include liquid hydrogen, liquid deuterium, carbon, iron and tungsten. The target rotary system also contains an empty liquid target flask and a "no target" position used for background subtraction. The muons generated from this interaction then enter the first dipole-a closed-aperture, solid iron magnet (FMAG) serving three primary purposes: it focuses high energy muons while giving them a $p_{T}$ kick of $\sim 3.0 \mathrm{GeV} / \mathrm{c}$, it functions as a beam dump for the protons in the beam that do not interact with the target material and it serves as a muon filter by inhibiting hadronic debris from further traversing the spectrome-ter. The downstream magnet is a large, open-aperture magnet (KMAG) and gives an additional $p_{T}$ 


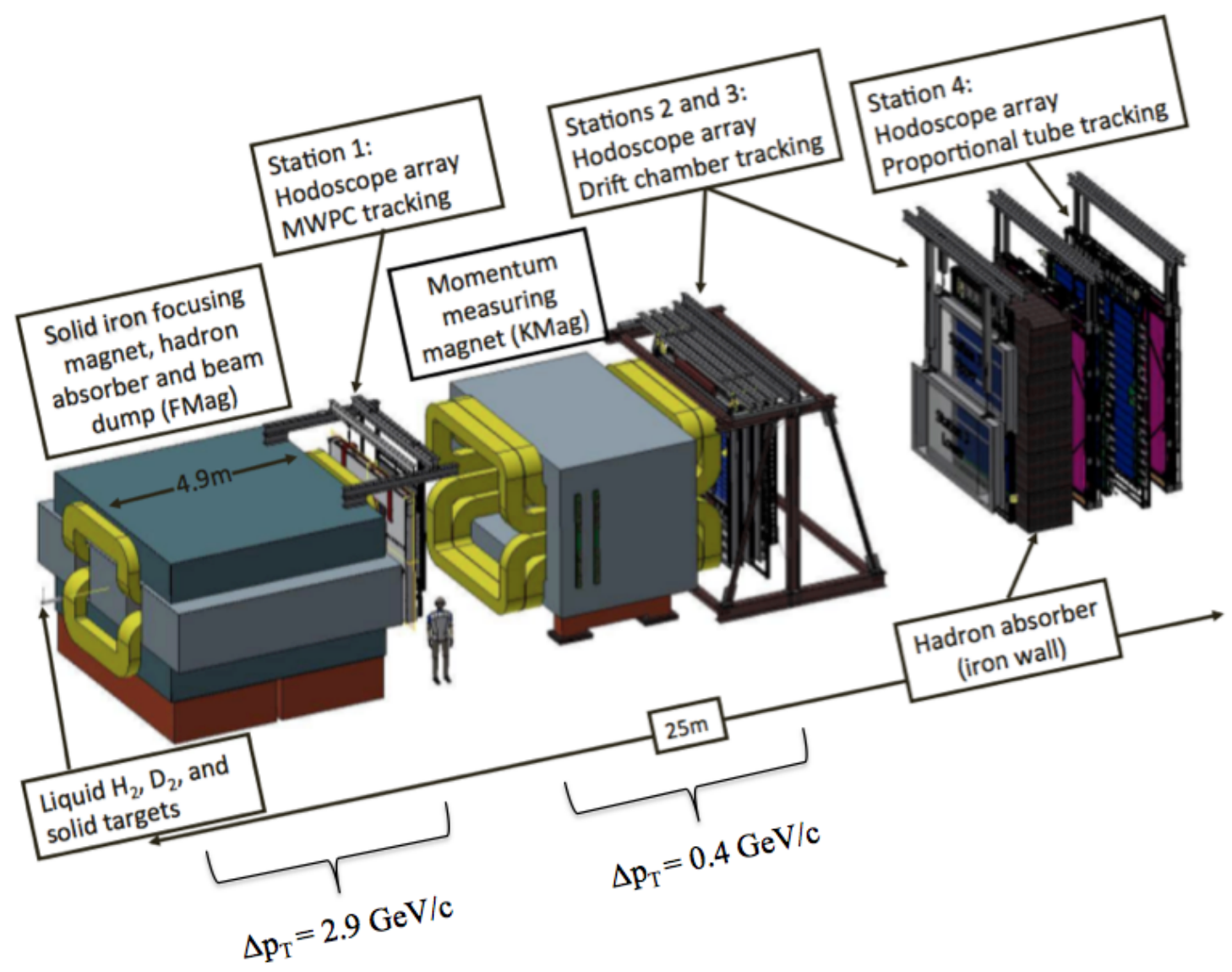

Figure 2.1: The SeaQuest Spectrometer [29]. 
kick of $\sim 0.4 \mathrm{GeV}$ to the focused muons. The detector system of the spectrometer is composed of an array of 4 stations, each of which contains either drift chambers or proportional tube detectors for tracking, in addition to segments of hodoscopes, which provide fast information for an FPGA-based road look up. In the middle of Station 3 and Station 4 , an iron absorber wall of $\sim 1$ meter in length is used to absorb any remaining strongly interacting particles that make it through the 3 stations. At the tail end of the spectrometer resides the muon identification station (Station 4), comprised purely of proportional tubes and hodoscopes. In the ensuing sections, a detailed description of all principal sub-systems of the spectrometer will be reported. It is important to note that throughout the remainder of this thesis, the coordinate system convention referenced will be the following: the positive z-axis lies along the proton beam direction and the positive y-axis points vertically upward, while the positive $\mathrm{x}$-axis is to the left of the beam in order to complete a right-handed Cartesian system with the origin.

\subsection{Proton beam production}

SeaQuest employs a 120-GeV proton beam delivered by Fermilab's Main Injector in "slow spills", each spill lasting four seconds with a one minute lag between spills. Fig. 2.2 shows the accelerator complex at Fermilab where the Main Injector is located, a two-mile circumference ring capable of accelerating the $8 \mathrm{GeV}$ beam of protons received from the Booster to an energy of $120 \mathrm{GeV}$. Here, the beam is extracted using a resonance process described in detail in this section, along with the function of the relevant sub-systems for this process shown in Figure 2.2.

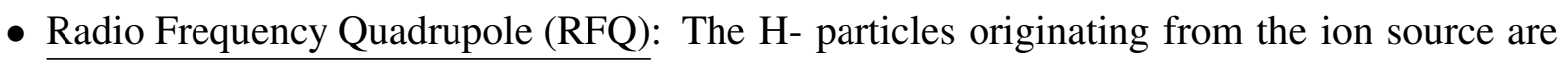
accelerated by the RFQ to $\sim 35 \mathrm{KeV}-750 \mathrm{KeV}$. The proton beam acquires a $53.1 \mathrm{MHz}$ $\mathrm{RF}$ (Radio Frequency) structure in the RFQ, which it retains throughout its lifespan. This is accomplished via shaped electrodes modulating the electromagnetic standing wave fields to allow for transverse focusing, longitudinal focusing (bunching), and acceleration all in one device. The low energy beam is then sent through the LINAC. 
- Linear Accelerator (LINAC): The LINAC, a 500-foot straight accelerator, takes the particles and accelerates them to about $400 \mathrm{MeV}$. Before entering the booster ring, the $\mathrm{H}$ - ions pass through a carbon foil to become $\mathrm{H}+$ ions (protons).

- $\underline{B o o s t e r}:$ The protons enter a circular accelerator of $\sim 1,500$ feet in circumference called the Booster which further accelerates the beam to $8 \mathrm{GeV}$. The particles travel around the Booster about 20,000 times in 33 milliseconds before they get transferred to the recycler.

- Recycler Ring (RR): The RR is a 2-mile circumference ring that serves as a stopping place for the beam before enter the Main Injector. At this stage, the beam is combined into batches of protons to form a greater intensity beam. During this "slip stacking" process, the beam intensity fluctuates greatly between empty RF buckets to high intensity RF buckets with a transverse structure that is highly volatile.

- Main Injector (MI): Once the beam is in the MI, it gets ramped up from $8 \mathrm{GeV}$ to 120 GeV. An electro-magnetic septum gradually scrapes off beam and splits it into manifolds with the use of powerful electric fields. Slices of the transversely oscillating beam then begin to be distributed to several beamlines dedicated for the fixed-target experiments and the Fermilab Test Beam Facility. SeaQuest uses the last two sections of the Neutrino Muon (NM) beamline, NM3 and NM4.

\subsubsection{Beam structure}

As previously cited, the beam delivered to SeaQuest consists of a four-second "spill" each minute; throughout the remaining $56 \mathrm{~s}$, beam pulses are delivered to the Neutrinos at the Main Injector (NuMI) target for the neutrino experiments. During the four-second pulse, the beam is microscopically composed of individual beam buckets about $1 \mathrm{~ns}$ in length separated by $18 \mathrm{~ns}$; this reflects the underlying $53 \mathrm{MHz}$ RF structure of the MI. One could visualize the buckets within each spill as a cylindrical-like cloud of protons measuring close to $20 \mathrm{~cm}$ in length, with a diameter of about $1 \mathrm{~cm}$. Figure 2.3 shows a diagrammatic representation of the micro-structure of the beam. While the 


\section{Fermilab Accelerator Complex}

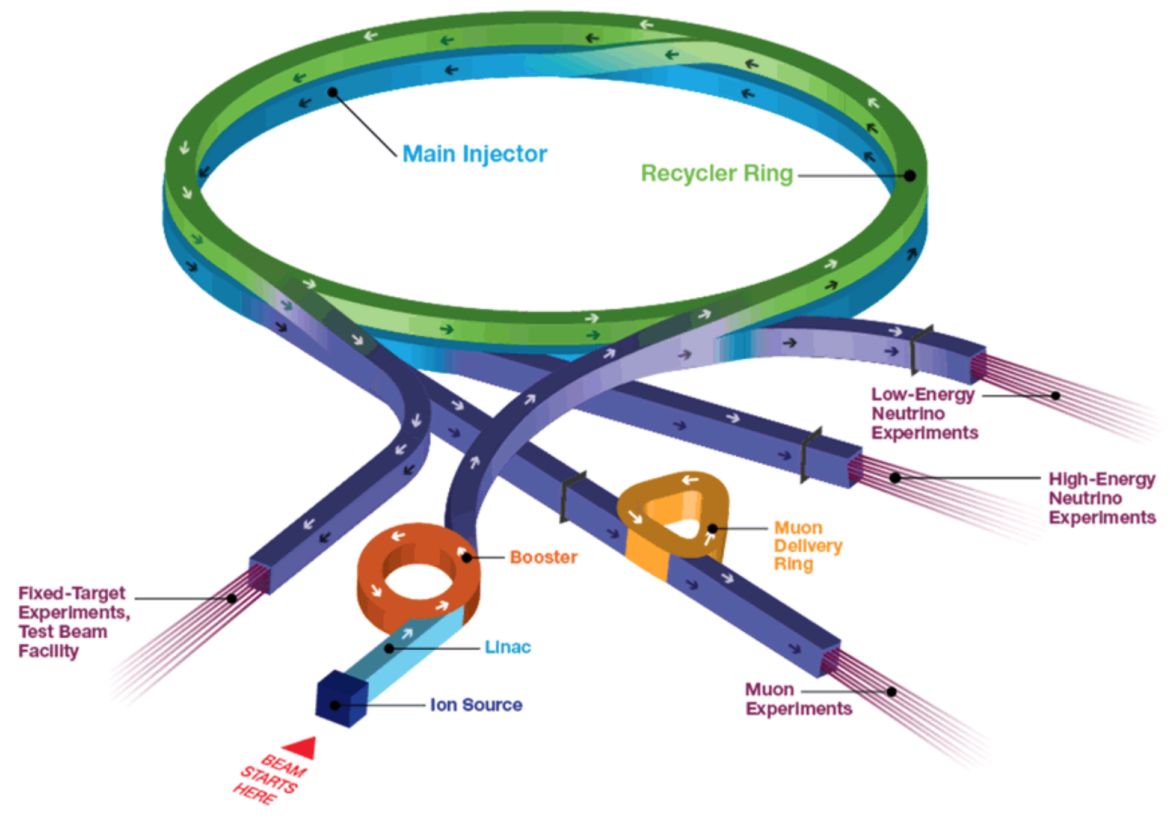

Figure 2.2: Fermilab Accelerator Complex [30]

MI can sustain as many as 7 "booster batches" of 84 buckets, only 6 batches are run in order to allow injections and extractions from the Booster, without disturbing the booster batches already within the MI. A short "abort gap" via empty buckets is also implemented into the beam profile to allow time for the diversion of the beam from the MI into the dump. The beam intensity at SeaQuest is not uniform in time, as the intensity of different buckets can change considerably over a spill. Normally, 492 of the 588 RF buckets in the MI contain protons during the slow spill cycle but the number of protons in these 492 buckets varies greatly throughout a slow spill as seen in Figure 2.5, which also shows a sample output of the Beam DAQ Cerenkov counter. For extremely high-intensity buckets, a "splat" effect can occur where the spectrometer gets flooded with a significant number of background tracks that saturate detectors and can fool the trigger system into thinking it has seen a good dimuon event. A "Beam Intensity Monitor" was designed to address this issue and will be described in the following section. 


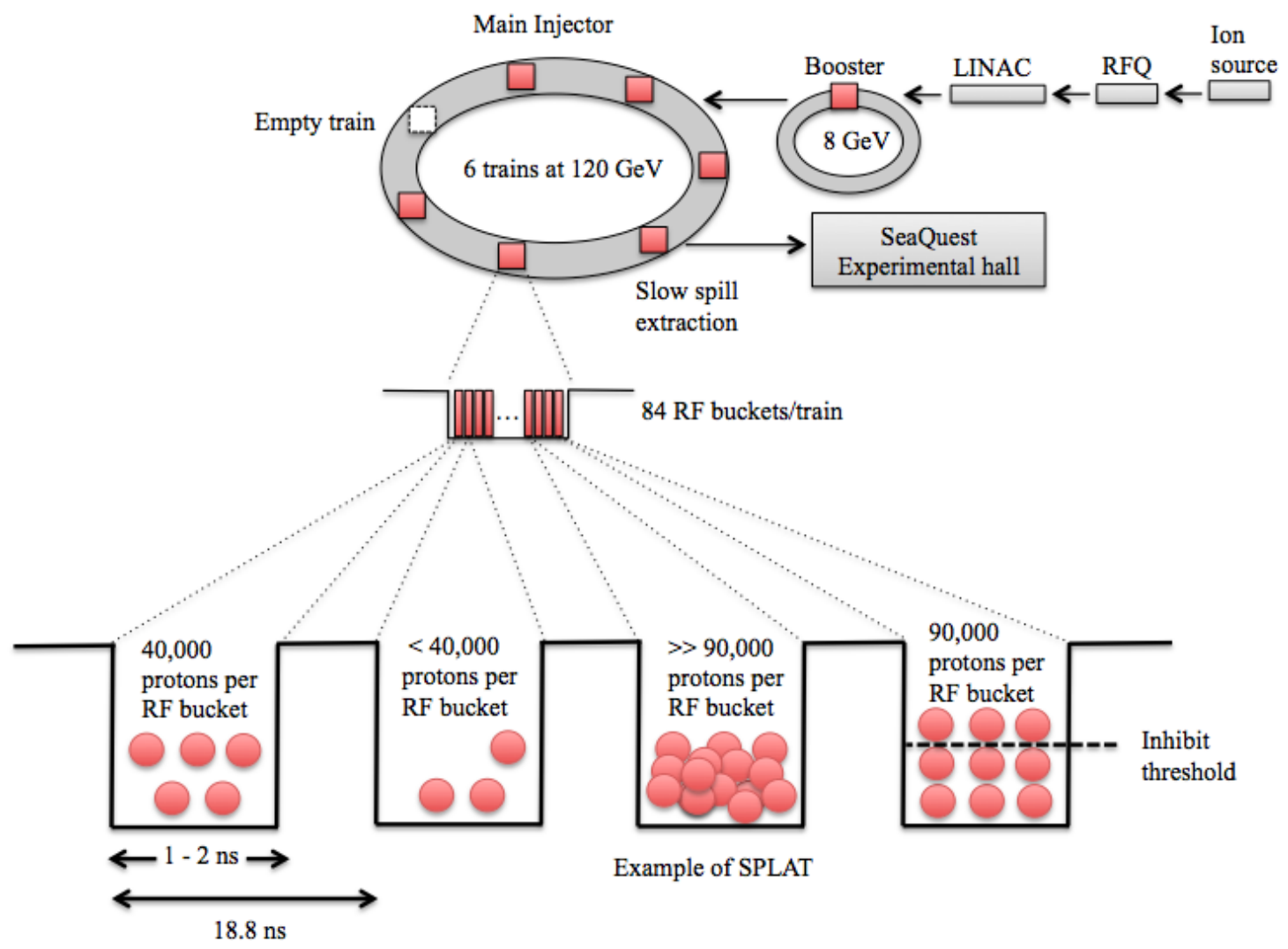

Figure 2.3: Micro structure of the beam along with varying intensity buckets from [4]. 


\subsection{Beam monitoring}

As the injected, fully formed beam traverses the beamline, it encounters different detectors intended for monitoring purposes before reaching SeaQuest. Among these are segmented wire ionization chambers (SWICs), which measure position and size of the beam on a spill-by-spill basis, and are also used for beam tuning. There are two types of monitors dispersed along the beam line, which measure the intensity profile of the beam: ion chambers (ICs) and secondary emission monitors (SEMs). Normalization of both of these detectors is accomplished via activation measurements of thin copper foils, positioned along with the beam, with known cross-sections. SEMs measure intensity by counting the number of electrons knocked off from their internal foils by the passing beam and are frequently used in high-energy experiments. They are the preferred detector for these measurements because, unlike the IC, SEMs do not saturate at high intensity and their response is typically linear over a large range, particularly the one measured at SeaQuest. However, they do not have individual bucket resolution. At SeaQuest, the readout of the SEM in the G2 enclosure (referred to later as G2SEM) is used to evaluate the number of protons received over the span of the $4 \mathrm{~s}$ spill.

\subsubsection{Beam intensity monitor}

Joining what is referred to as the "upstream instrumentation package" of the beamline, which both the SWICs and the SEMs represent, is the SeaQuest Beam Intensity Monitor (BIM). This particular monitor is designed to inhibit triggers when a bucket exceeds a preset threshold; this inhibit threshold is set to 95,000 protons per RF bucket. The BIM has two main features: the gas Cerenkov counter and the QIE, a charge integrator and encoder module. The gas Cerenkov counter, shown in Figure 2.4, measures the beam intensity with the use of a gaseous Cerenkov radiator; a gas mixture maintained at atmospheric pressure made of $80 \%$ Argon and $20 \% \mathrm{CO}_{2}$. The baffle, seen in the diagram, is made from black construction paper and is held parallel to the mirror, an aluminized Kapton plate held on an elliptical G10 frame guiding the light to a single photomultiplier tube, 


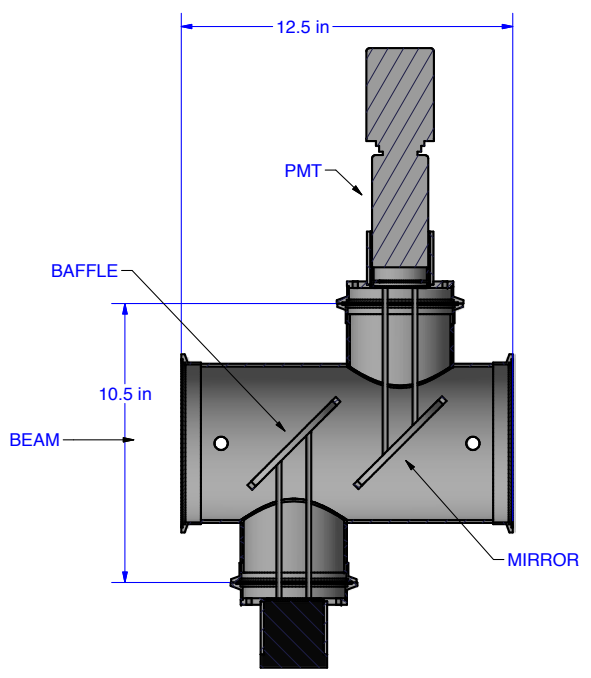

Figure 2.4: The Beam Intensity Monitor (BIM) Čerenkov counter. [29]

which in turn collects all of the Cerenkov light that falls on the phototube's face. This parallel setup is specifically curated to maintain the independence of the proton path length in the radiator from that of the beam position and to ensure that the light produced before the baffle is fully obstructed by it. In order to remain in the linear dynamic range and to produce suitable signal amplitude for the QIE module, the 8-stage photomultiplier tube is biased at $-870 \mathrm{~V}$. The signal from the photomultiplier tube is collected and delivered to the QIE module, a custom integrated circuit designed to integrate and digitize the signal. The module is synchronized to the MI RF clock and is able to do ADC conversions every $18.8 \mathrm{~ns}$ on the input current. This setting allows for intensity measurements to span from 30,000 protons to more than 106,000 protons per bucket. Along with monitoring beam intensity, the BIM interface module also offers information that is imperative to the analysis and operations of the experiment including: a complete spill intensity sum $\left(Q I E_{\text {sum }}\right)$, an integrated beam measurement during trigger dead time (trigger_sum_no_inhibit), an intensity sum when the trigger inhibits were delivered (inhibit_block_sum), a beam-intensity record of buckets close to the triggered bucket, including up to 16 buckets before and after the triggered bucket and a full documentation of the bucket-by-bucket intensity for the spill. The specific purpose of many of these values provided by the BIM will be highlighted throughout the thesis, particularly in the main analysis section. 

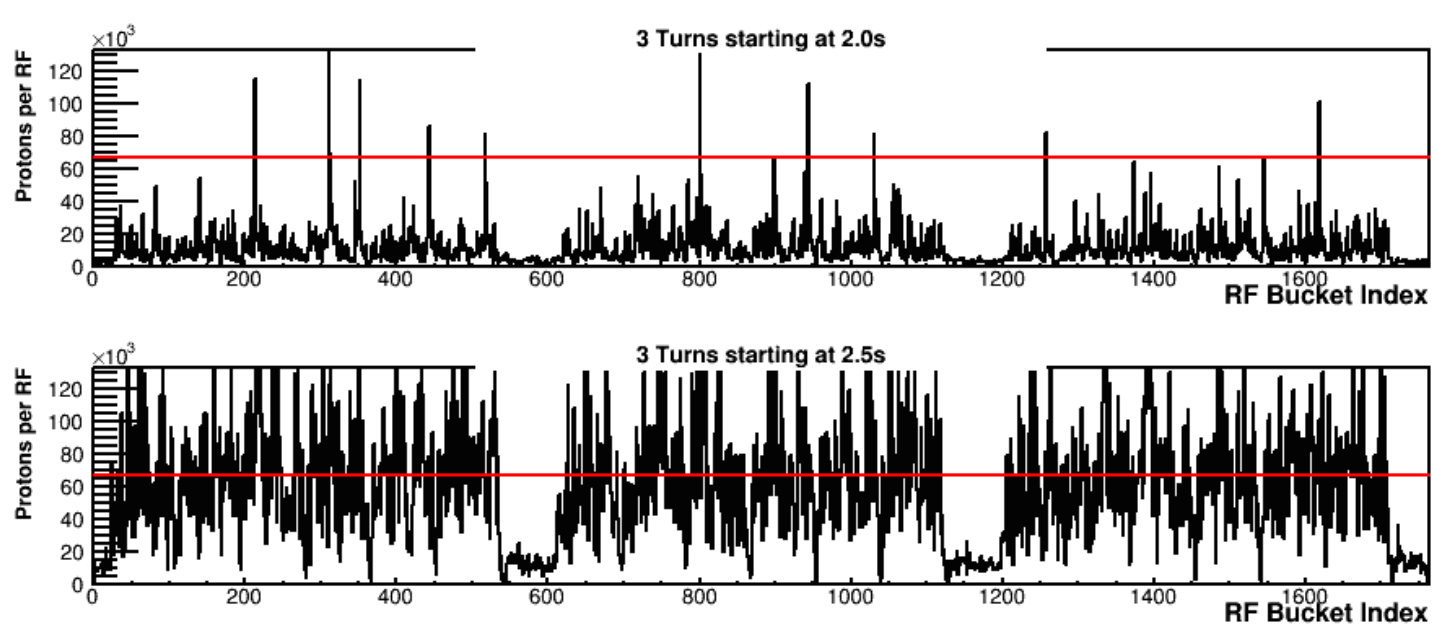

Figure 2.5: The beam intensity measured by the Beam DAQ Cerenkov counter every beam bucket. Each strip shows the number of protons per beam bucket as a function of time. The red line in each plot denotes the threshold above which the trigger is inhibited. [29]

\subsection{Targets}

Downstream of the BIM, about $50.8 \mathrm{~cm}$ upstream from the first surface of FMAG, is the "target cave" where the primary SeaQuest target system is located. Much of the target prototype was adopted from the E866/NuSea experiment, along with some recycled parts. The general layout is shown in Figure 2.6.

\begin{tabular}{|c|c|c|c|c|c|}
\hline Position & $\begin{array}{c}\text { Target } \\
\text { Material }\end{array}$ & $\begin{array}{c}\text { Target } \\
\text { Density } \\
\left(\mathrm{g} / \mathrm{cm}^{3}\right)\end{array}$ & $\begin{array}{c}\text { Thickness } \\
(\mathrm{cm})\end{array}$ & $\begin{array}{c}\text { Number of } \\
\text { Interaction } \\
\text { Lengths }\end{array}$ & $\begin{array}{c}\text { Typical } \\
\text { Spills/ } \\
\text { Cycle }\end{array}$ \\
\hline 1 & $\mathrm{LH}_{2}$ & 0.071 & 50.8 & 0.069 & 10 \\
\hline 2 & Empty Flask & - & - & 0.0016 & 2 \\
\hline 3 & $\mathrm{LD}_{2}$ & 0.163 & 50.8 & 0.120 & 5 \\
\hline 4 & No Target & - & - & 0 & 2 \\
\hline 5 & Iron & 7.87 & 1.905 & 0.114 & 1 \\
\hline 6 & Carbon & 1.80 & 3.322 & 0.209 & 2 \\
\hline 7 & Tungsten & 19.30 & 0.953 & 0.096 & 1 \\
\hline
\end{tabular}

Table 2.1: Features of the targets at SeaQuest. The "Spills/Cycle" values shown represent default configurations. Values can change depending on the response to sample balancing needs and running configurations [29].

SeaQuest employs 7 different target positions: two liquid targets, three solid targets, an empty 


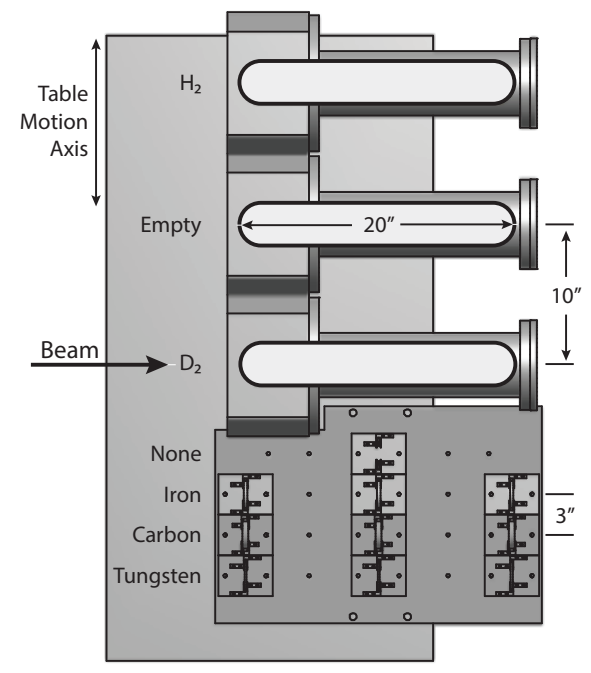

Figure 2.6: Diagrammatic layout of mobile target table showing all 7 SeaQuest targets (top view) .

flask, and an empty solid target holder, labeled "none" target; the latter two positions are used for background estimations. The targets can be rotated along the x-direction (perpendicular to the beam) over a range of $91.4 \mathrm{~cm}$ with the use of a remotely positionable table; a single step of the motor moves the table by $2.54 \mu \mathrm{m}$. Magnetic proximity sensors fastened to the target table monitor all positions of the targets, each recalibrated every time the table passes through the central proximity sensor. As reported in Table 2.1, data is recorded on different targets in a cyclical order and can be programmed to take a specific amount of data per target. This target rotation is done in nominal data taking conditions to reduce the systematics related to long-term changes in experimental conditions, like differences in detector acceptances or beam quality. As part of the work completed for this dissertation, the maintenance, troubleshooting and monitoring of the target system was supported by me and University of Michigan alumni, Bryan Ramson.

\subsubsection{Solid targets}

The geometry of each solid target is that of three identically shaped, 2-inch diameter disks. The properties of these targets are reported in Table 2.1, where the true thickness of each disk is $1 / 3$ of the listed value. The target disks are placed $25.4 \mathrm{~cm}$ apart along the beam axis (with the exception 
of iron $(17.1 \mathrm{~cm})$ during Run-II data taking) on the beam axis. This was done to homogenize the spatial distribution of the solid and liquid targets as much as possible, thus minimizing target dependent variations in spectrometer acceptance.

\subsubsection{Cryogenic targets}

The liquid targets are primed using gaseous hydrogen and deuterium, which are turned into liquid using a closed-circuit helium refrigeration system. An "Ultra High Purity" gas, 99.999 \% commercially pure, was used to produce liquid Hydrogen $\left(\mathrm{LH}_{2}\right)$. The gas used to produce liquid deuterium $\left(L D_{2}\right)$ target came from two sources:

- A gas that was used for bubble chamber experiments at Fermilab (purity: $95.8 \pm 0.2 \%$ ). Gas contamination from: ${ }^{2} \mathrm{H}$ and ${ }^{1} \mathrm{H}$ in $\mathrm{HD}$ molecules.

- Commercially available deuterium used towards the end of the experiment (purity: 99.99\%).

Each refrigerator of the cooling system is comprised of a Cryomech water-cooled compressor and cold head, capable of approximately $25 \mathrm{~W}$ of cooling power at $20 \mathrm{~K}$. Temperature sensitive resistors are used to monitor the level of the liquid during filling and data-taking. As each liquid type gets respectively filled into the cylindrically shaped flask, an insulating vacuum vessel is used to minimize the heat load surrounds the flasks. The flask has hemispherical end-caps and the thickness of the flask wall and the end-cap are $67 \mu \mathrm{m}$ and $51 \mu \mathrm{m}$, respectively. Each of the flasks are high-pressure tested and leak-checked, capable of holding 2.2 liters of liquid. Both targets take under 19 hours to fill.

Throughout data taking, the liquid targets are maintained along the vapor-liquid saturation curve and the vapor pressure, along with the temperature in the flask are continually monitored. Variations in pressure and temperature measurements are used to estimate the uncertainty in the density.

In Figure 2.7, an illustrative diagram of the flask and high vacuum plumbing is shown. The diffusion pump, supported by a mechanical fore pump, conserves an insulation vacuum during 
regular operations. The mechanical rough pump has two functionalities: to purge the target flask prior to filling and to serve as a secondary pump, if there are any issues with the diffusion pumping system. On the supply and vent lines, Setra pressure transducers monitor the flask pressure and Cernox temperature sensors monitor Cold-head temperatures. Thermocouple gauges on the $\mathrm{H}_{2}$ cart record convection vacuum gauges and fore and rough vacuums on the $\mathrm{D}_{2}$ pump cart.

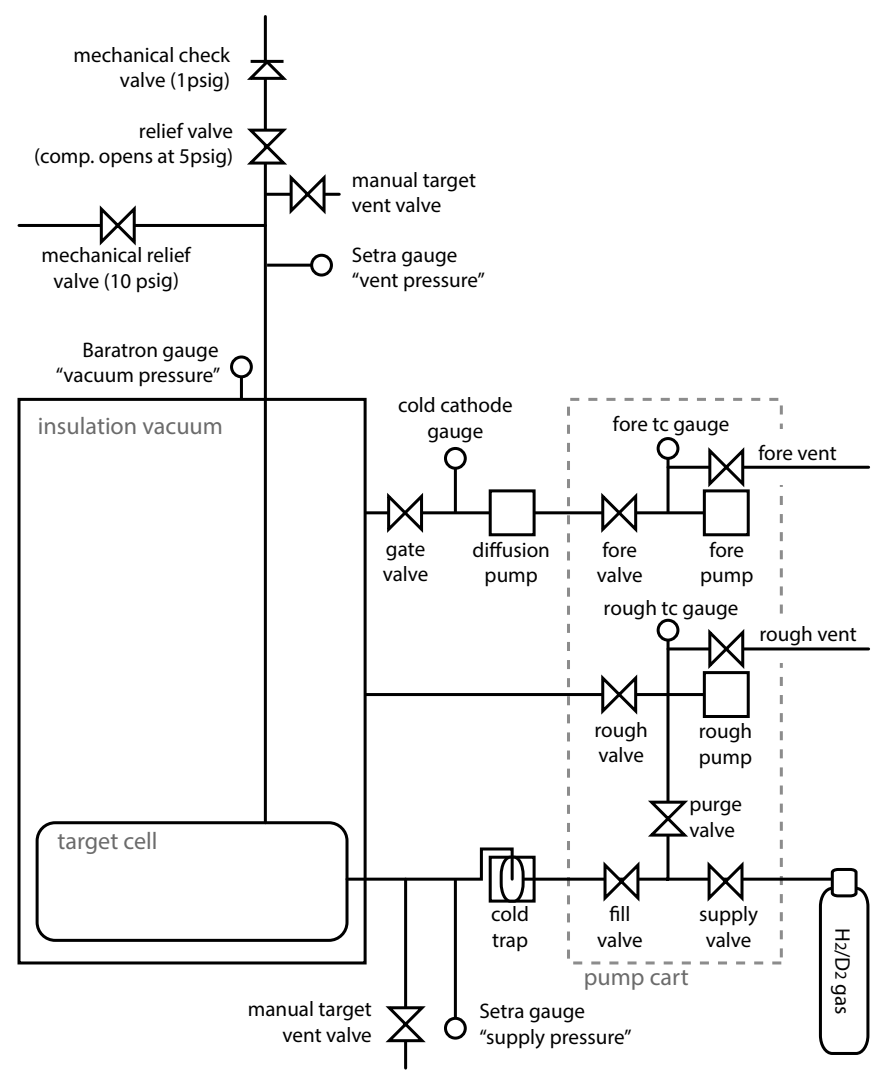

Figure 2.7: Schematic depiction of the flask and vacuum plumbing for the two cryogenic targets [29].

\subsection{Magnets}

Two dipole magnets, referred to as FMAG and KMAG, are fundamental to the SeaQuest spectrometer. The fields generated by the two magnets, which are configured to point in the same vertical direction $(+\mathrm{Y}$ or $-\mathrm{Y})$ in nominal running conditions, result in a horizontal bend $(+\mathrm{X}$ or $-\mathrm{X})$ by the incoming muons as they pass through them. This specific two-magnet setup makes the SeaQuest 
spectrometer a "focusing spectrometer", one that is able to sweep out low momentum muons and push higher momentum muons into acceptance. For convention, the XZ-plane is referred to as the "bend plane" and the YZ-plane as the "non-bend plane".

The magnet immediately following the target cave is FMAG, a solid iron A-frame magnet made from $43.2 \mathrm{~cm}$ x $160 \mathrm{~cm}$ x $503 \mathrm{~cm}$ high-purity iron slabs. The iron was recycled from the Columbia University Nevis Laboratory Cyclotron in 1980 and its three aluminum "bedstead" coil sets, seen in yellow in Figure 2.8, were recovered from the E866 SM3 magnet. A 1.9 T central magnetic field and a total transverse momentum deflection of $\sim 3.0 \mathrm{GeV}$ is achieved by exciting FMAG's coil to the nominal setting of $2000 \mathrm{~A}$ at $25 \mathrm{~V}$ using $50 \mathrm{~kW}$ of power. During operations, FMAG uses blocks of iron to absorb produced hadrons along with the beam that did not interact with the target. To inhibit the deposited beam from back "splashing", a hole $25 \mathrm{~cm}$ deep (5 cm in diameter) was drilled into the upstream surface of the central iron slab, along the beam axis. This creates enough distance between the initial interactions of the residual protons and the targets, minimizing the possibility of muon misidentification. FMAG's calibration was realized with the reconstruction of $J / \psi$ mass and a magnetostatic modeling program was used to model the magnetic field distribution inside. The current flowing through FMAG was monitored by the Fermilab accelerator control system and its excitation status was recurrently broadcasted, in order to prevent damage from the beam to the spectrometer while the magnet is off.

The downstream magnet is KMAG, a $300 \mathrm{~cm}$ long iron rectangular air-core magnet with 289 $\mathrm{cm}$ x $203 \mathrm{~cm}$ high central gap, as seen in Figure 2.9. It was made from donated steel from the University of Maryland Cyclotron and was originally constructed by the E799/KTeV collaboration at Fermilab. KMAG's main function is to provide muon momentum measurements. During data taking periods, KMAG was excited to a current of $1600 \mathrm{~A}$ at $270 \mathrm{~V}$ using $430 \mathrm{~kW}$ of power, which generated a magnetic field of $0.4 \mathrm{~T}$ and a total magnetic deflection of $0.39 \mathrm{GeV} / \mathrm{c}$. The KTeV group had already measured the magnetic field distribution and the SeaQuest group verified the central field calibration with a Hall probe, while the final value for the magnetic field was determined in the same way as FMAG's. 

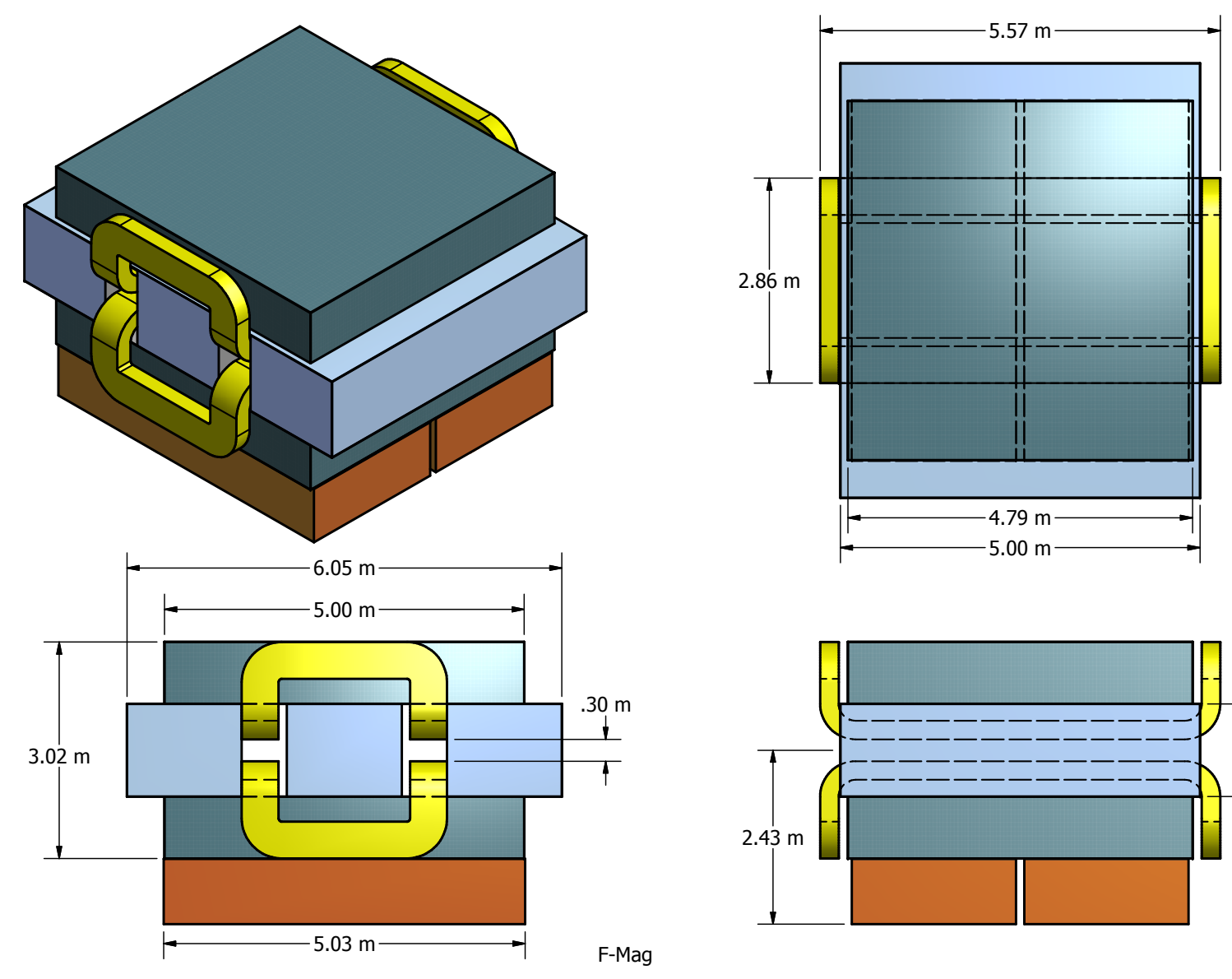

Figure 2.8: FMAG schematic view. 

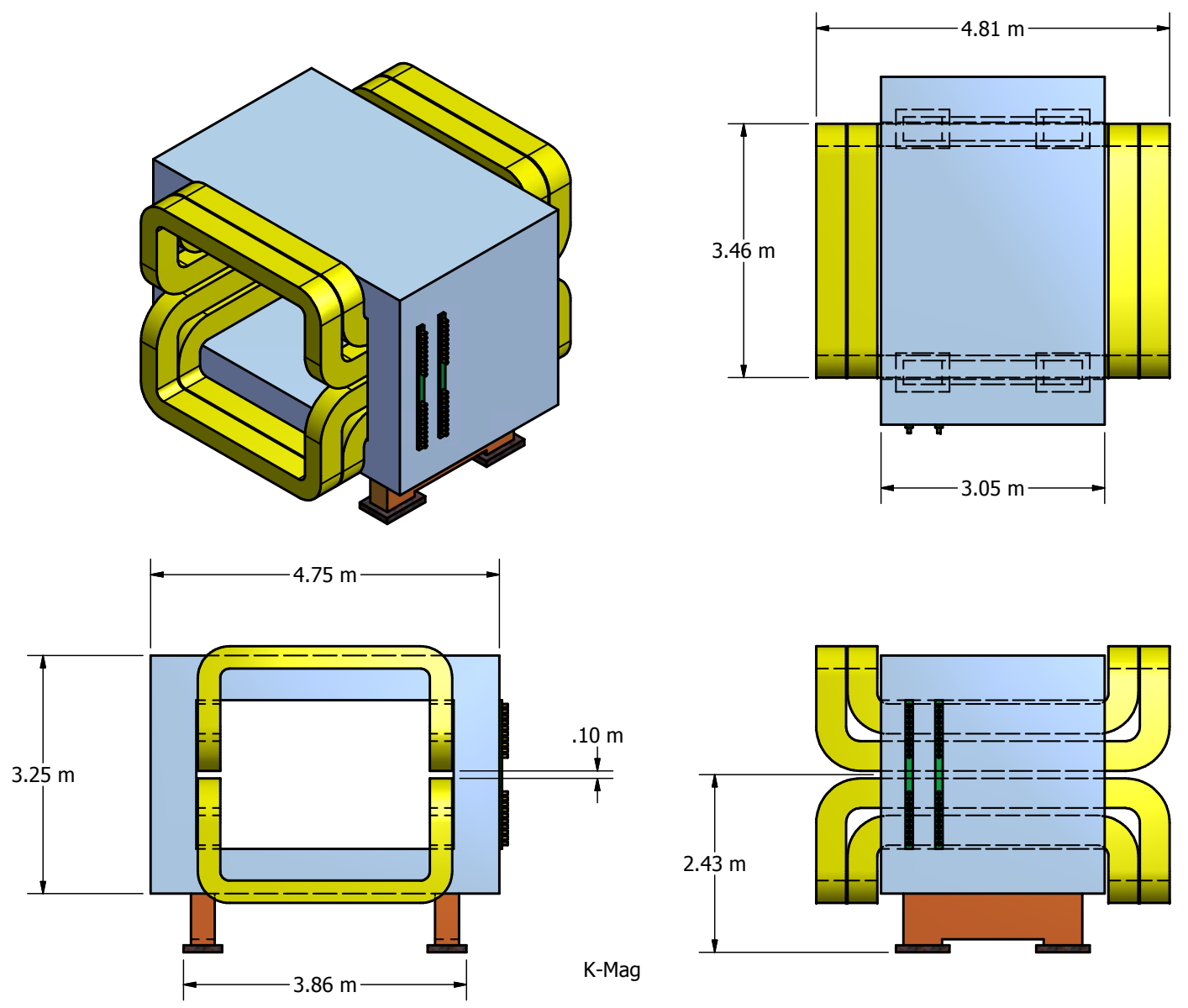

Figure 2.9: KMAG schematic view. 


\subsection{Tracking Detectors}

\subsubsection{Hodoscopes}

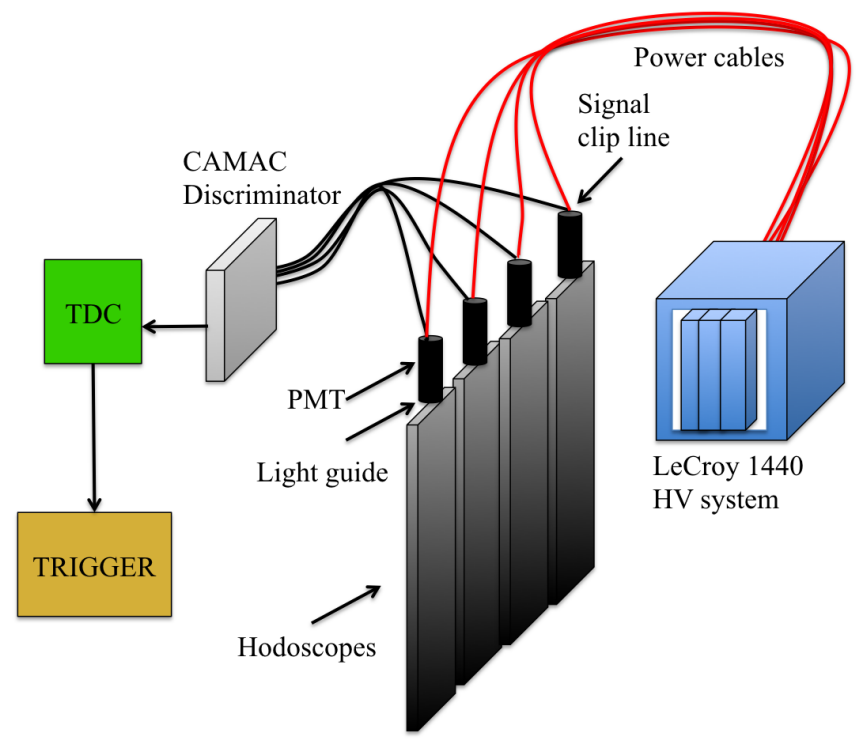

Figure 2.10: Diagram of hodoscopes and its features.

SeaQuest uses plastic scintillator hodoscope planes for the primary trigger of the spectrometer in all four stations. Hodoscopes in Stations 1 and 2 were recycled from HERMES while the last two stations used new Eljen EJ-200 scintillator material. The planes of the hodoscopes, which consist of scintillator paddles, come in two kinds of spatial configurations: vertically oriented (xplane) and horizontally oriented (y-plane), each respectively measuring the $\mathrm{x}$ and $\mathrm{y}$ position of passing particles. Stations 1 and 2 each have an x-plane and a y-plane, station 3 has one x-plane and station 4 has one x-plane and two y-planes. All planes have a slight overlap of $0.32 \mathrm{~cm}$ with the adjacent plane to make sure there are no breaches in the acceptance. Each plane is divided in the middle and regions are labeled as follows: T/B (for Top/Bottom) denoting the $+y /-y$ half for the $\mathrm{X}$-planes, and L/R (for Left/Right) denoting the $+\mathrm{X} /-\mathrm{X}$ half for the $\mathrm{y}$-planes. Spatial specifications of each configuration are shown in Figures 3.11 - 3.17. Table 2.2 gives the number of scintillators, their physical sizes, and total aperture for each plane. 
As seen in Figure 2.10, each hodoscope paddle is attached to a plexi-glass light guide that is connected to a photomultiplier tube (PMT); the hodoscopes as a whole are covered with black paper to avoid light exposure. Station 4 is the only station that has PMTs mounted on both ends of the scintillator paddles; all others have only one PMT on the outer end of each paddle. This configuration for Station 4 is made to reduce the time for the light to travel in the paddle. As charged tracks pass through the hodoscopes, PMTs collect the light signals produced in the scintillators and generate analog pulse outputs. The width of the pulses is reduced by "clip lines" attached to the PMT bases to a full width of about 10-15 ns. These output pulses are then processed through CAMAC discriminators, digitized by Time-to-Digital Converters (TDCs), and ultimately transmitted to the NIM- and FPGA-based trigger system.

\begin{tabular}{c|c|c|c|c|c}
\hline \hline Detector & $\begin{array}{c}\text { Paddle width } \\
(\mathrm{cm})\end{array}$ & $\begin{array}{c}\text { Paddle length } \\
(\mathrm{cm})\end{array}$ & \# of paddles & $\begin{array}{c}\text { Width } \times \text { Height } \\
(\mathrm{cm})\end{array}$ & Z - position \\
\hline \hline H1T & 7.32 & 69.9 & 23 & $162 \times 69.85$ & 667.12 \\
H1B & 7.32 & 69.9 & 23 & $162 \times 69.85$ & 667.12 \\
H1L & 7.32 & 78.7 & 20 & $78.74 \times 140.12$ & 654.03 \\
H1R & 7.32 & 78.7 & 20 & $78.74 \times 140.12$ & 654.03 \\
H2T & 13.04 & 132 & 16 & $203.24 \times 150.00$ & 1421.06 \\
H2B & 13.04 & 132 & 16 & $203.24 \times 150.00$ & 1421.06 \\
H2L & 13.07 & 152 & 19 & $132.00 \times 241.29$ & 1402.86 \\
H2R & 13.07 & 152 & 19 & $132.00 \times 241.29$ & 1402.86 \\
H3T & 14.59 & 132 & 16 & $227.52 \times 167.64$ & 1958.51 \\
H3B & 14.59 & 132 & 16 & $227.52 \times 167.64$ & 1958.51 \\
H4T & 19.65 & 182.9 & 16 & $304.52 \times 182.88$ & 2234.50 \\
H4B & 19.65 & 182.9 & 16 & $304.52 \times 182.88$ & 2250.68 \\
H4Y1L & 23.48 & 152.4 & 16 & $152.40 \times 365.80$ & 2130.27 \\
H4Y1R & 23.48 & 152.4 & 16 & $152.40 \times 365.80$ & 2146.45 \\
H4Y2L & 23.48 & 152.4 & 16 & $152.40 \times 365.80$ & 2200.44 \\
H4Y2R & 23.48 & 152.4 & 16 & $152.40 \times 365.80$ & 2216.62 \\
\hline \hline
\end{tabular}

Table 2.2: Information on different hodoscope planes. The designation (L) and (R) refer to beam left or right and (T) and (B) refers to Top and Bottom. $\mathrm{Z}$ - position is measured from the front face of FMAG. [18]. 


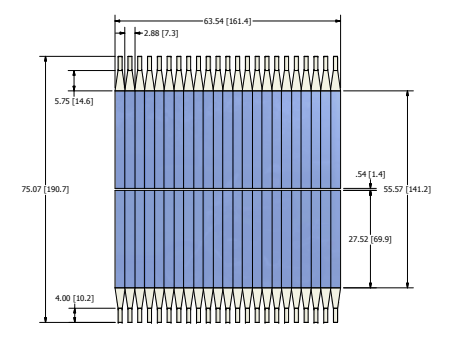

(a) H1X Hodoscope panels at St 1.

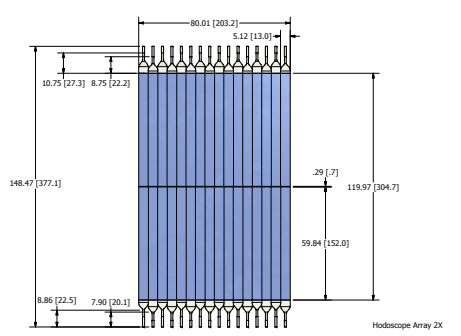

(c) H2X Hodoscope panels at St 2.

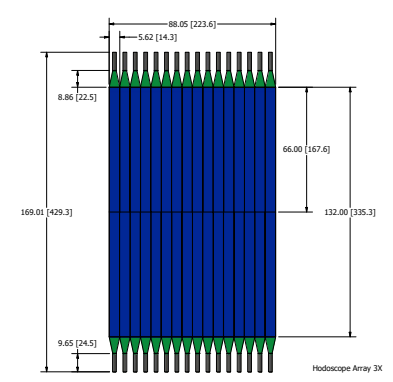

(e) H3X Hodoscope panels at St 3.

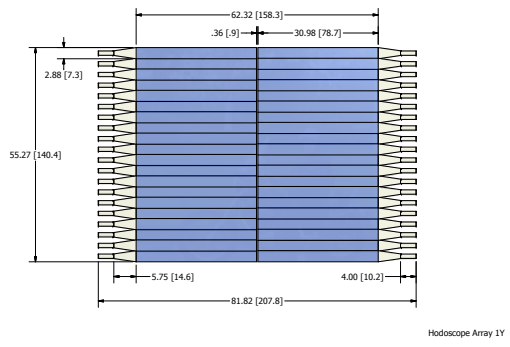

(b) H1Y Hodoscope panels at St 1.

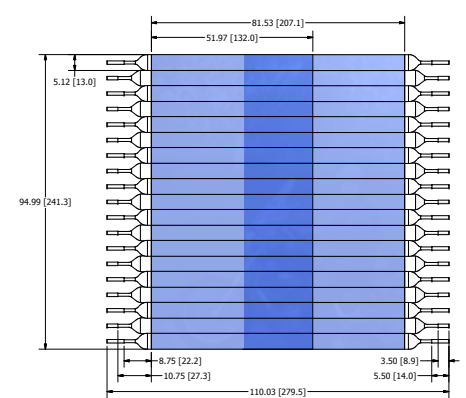

(d) H2Y Hodoscope panels at St 2.

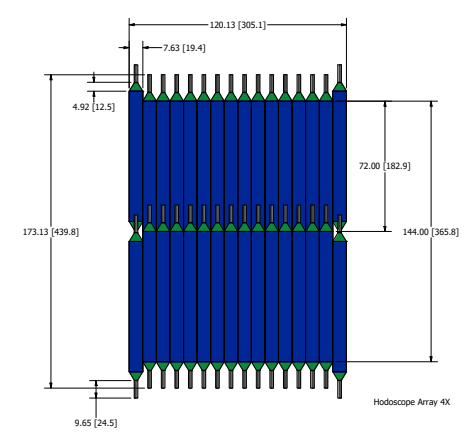

(f) H4X Hodoscope panels at St 4.

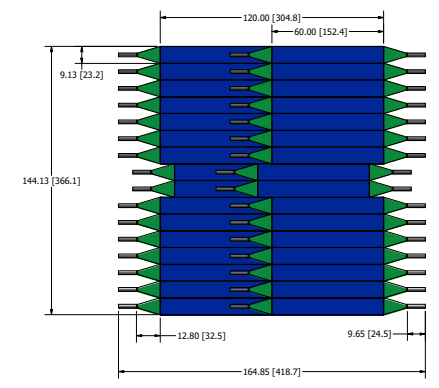

(g) H4Y Hodoscope panels at St 4.

Figure 2.11: SeaQuest Hodoscopes 


\subsection{Drift chambers}

SeaQuest uses drift chamber technology to measure the spatial position of muons. Moving charged particles will always interact electromagnetically with neighboring electrons and create electron/ion pairs along their path. Drift chambers in the SeaQuest spectrometer exploit this phenomenon by providing a gaseous medium for the incoming muons to interact with. The number of pairs created from the incoming muons depends on their energy and the kind of gas used; specifications for SeaQuest will be reported later on in this section. When an electric field is applied, the electrons will start to drift through the gas in the direction of the anode wire, resulting in repeated collisions with the atoms in the gas. Depending on the magnitude of the electric field, electrons can gain sufficient energy between collisions to knock off more electrons from the gas and those knocked off electrons can ionize more gas molecules. What results is a form of particle avalanche resulting in an exponential upsurge of electrons. A drop-like avalanche develops surrounding the anode wire as seen in Figure 2.12. The electrons are quickly collected while the ions begin drifting towards the cathode generating the signal at the electrodes. The signal is the result of a voltage drop caused by the drift positive charge towards the cathode pulling stored energy from the anode. The induced signal is proportional to the number of ions from the primary interaction and this is what is detected by the electronics.

The drift time of the electrons from an ionizing event is used to gather spatial information. Figure 2.13 shows how this measurement is done. The drift time is calculated from the time difference between the hodoscope signal and the anode signal using a scintillation counter, and this difference is subsequently used to calculate the detector position of the charged particle. This is done via a distance to time (RT) curve for each of the planes in the drift chambers along with the former timing information. A description of the overall design of the drift chambers and their configuration in the SeaQuest spectrometer will follow. As part of the work completed for this dissertation, I helped support the maintanence and troubleshooting of these chambers.

Drift chambers are installed in the first 3 stations, with Station 1 and Station 2 each containing 


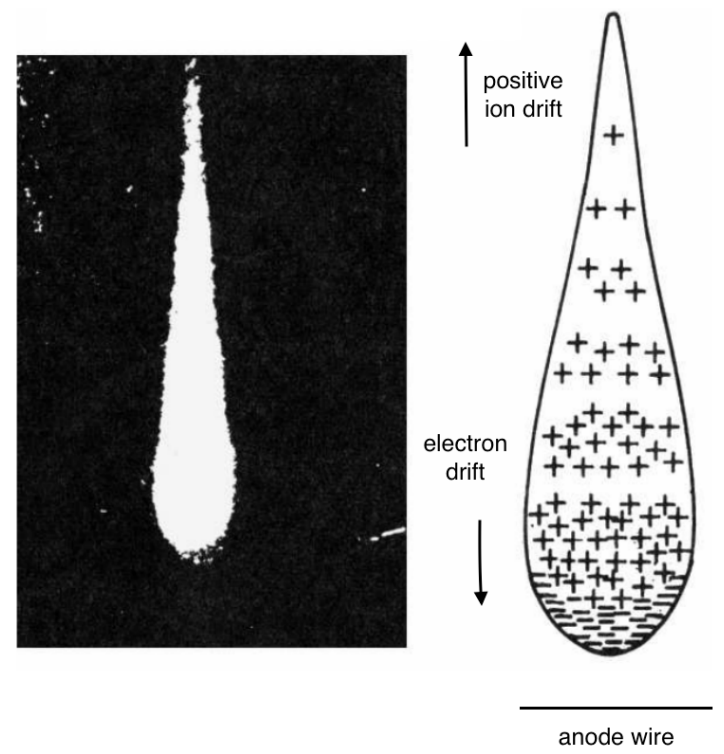

Figure 2.12: Drop-like shape of an avalanche. A cloud chamber picture (left) . A schematic view (right) [31].

one chamber (denoted D1 and D2, respectively), and Station 3 using two drift chambers in order to provide ample acceptance coverage. Chambers in Station 3 are referred to as D3p and D3m, where "p" and "m" refer to "plus" and "minus", where D3p provides the upper $(+Y)$ half measurement and D3m the lower (-Y) half. The chambers have a total of six wire planes: two wire planes measuring the $\mathrm{X}$-position along with two other planes measuring left and right stereo angles at $\pm 14^{\circ}$ (denoted $\mathrm{U}$ and V). The "primed" planes of each are right next to the "unprimed" planes but are transversely shifted by half of the drift cell width. There is a "left-right ambiguity" on fired wire in regards to which side the muon passed through that is resolved by this wire configuration. Key specifications to the drift chambers are reported in Table 2.3.

The drift chambers installed in the three stations are labeled DC1.1, DC1.2, DC2, DC3m.1, DC3m.2 and DC3p. The original drift chambers configuration during the commissioning run in 2012 was DC1.1 + DC2 + DC3m.1 + DC3p. However, the upper (DC3m.1) and lower (DC3p) halves at Station 3 were not symmetric in acceptance. In an effort to resolve this asymmetry, a new drift chamber, DC3m.2, was constructed at Fermilab. In Station 1, DC1.1 was also replaced with a 


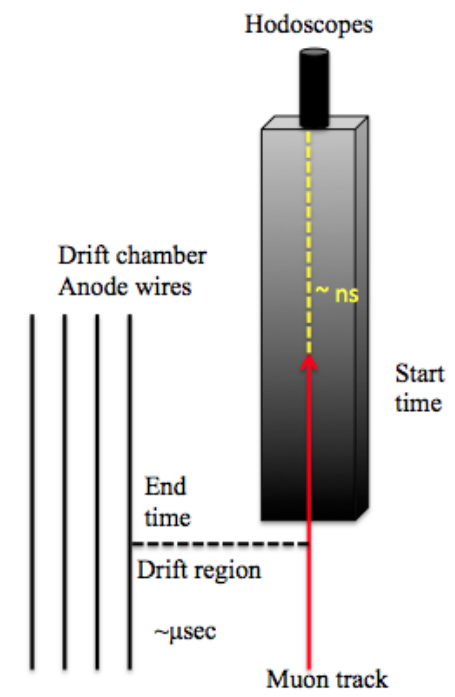

Figure 2.13: Diagram of drift time calculation.

new chamber (DC1.2) for Runs 4-6, improving rate-handling capabilities. Ultimately, DC1.1 was reinstalled and was kept running alongside DC1.2 due to intermittent issues with the new chamber. Table 2.4 summarizes all the Run configurations for the chamber.

\begin{tabular}{cc|cccc}
\hline Chamber & Plane & $\begin{array}{c}\text { Number } \\
\text { of wires }\end{array}$ & $\begin{array}{c}\text { Cell } \\
\text { width } \\
(\mathrm{cm})\end{array}$ & $\begin{array}{c}\text { Width } \\
\times \text { height } \\
(\mathrm{cm})\end{array}$ & $\begin{array}{c}z \text {-position } \\
(\mathrm{cm})\end{array}$ \\
\hline DC1.1 & X & 160 & 0.64 & $102 \times 122$ & 617 \\
& U, V & 201 & 0.64 & $101 \times 122$ & \pm 20 \\
DC1.2 & X & 320 & 0.50 & $153 \times 137$ & 617 \\
& U, V & 384 & 0.50 & $153 \times 137$ & \pm 1.2 \\
DC2 & X & 112 & 2.1 & $233 \times 264$ & 1347 \\
& U, V & 128 & 2.0 & $233 \times 264$ & \pm 25 \\
DC3p & X & 116 & 2.0 & $232 \times 166$ & 1931 \\
& U, V & 134 & 2.0 & $268 \times 166$ & \pm 6 \\
DC3m.1 & X & 176 & 1.0 & $179 \times 168$ & 1879 \\
& U, V & 208 & 1.0 & $171 \times 163$ & \pm 19 \\
DC3m.2 & X & 116 & 2.0 & $232 \times 166$ & 1895 \\
& U, V & 134 & 2.0 & $268 \times 166$ & \pm 6 \\
\hline
\end{tabular}

Table 2.3: Drift chamber specifications from [29].

All chambers, with the exception of D1.2, are filled with a gas mixture of Argon:Methane:CF4 (88\%:8\%:4\%). Given that the hit-rate of Station 1 is higher than the other stations, the ion drift velocity of the gas used in the first chamber should be higher, allowing for a faster response time and thus make for a better performing chamber. Originally, the proposed gas mixture for D1.2 


\begin{tabular}{c|c|c|c|c}
\hline \hline Run period & Dates & St 1 & St 2 & St 3 \\
\hline Run 1 & 2012 Mar - 2012 Apr & DC1.1 & DC2 & DC3p + DC3m.1 \\
Run 2 & 2013 Nov - 2014 Aug & DC1.1 & DC2 & DC3p + DC3m.2 \\
Run 3 & 2014 Nov - 2015 Jul & DC1.1 & DC2 & DC3p + DC3m.2 \\
Run 4 & 2015 Nov - 2016 Mar & DC1.2 & DC2 & DC3p + DC3m.2 \\
Run 5 & 2016 Mar - 2016 Jul & DC1.1 + DC1.2 & DC2 & DC3p + DC3m.2 \\
Run 6 & 2016 Nov - 2017 Jul & DC1.1 + DC1.2 & DC2 & DC3p + DC3m.2 \\
\hline \hline
\end{tabular}

Table 2.4: Configurations of Drift chambers used in different Runs [18].

was Argon:Isobutane:CF4:Methylal (68\%:13\%:16\%:3\%), given its drift velocity of a little over $50 \mu \mathrm{m} / \mathrm{ns}$. However, because of its superior gas gain, it was superseded by the mixture of Argon:Isobutane:CF4:Methylal (81\%:12\%:5\%:2\%). This new mixture is very flammable and was therefore only used in D1.2 as a contained safety measure.

The high voltages of the chamber were supplied by a group of NIM high-voltage modules located in the SeaQuest control room. Their magnitude was set to the lowest possible voltage on the limit of the efficiency plateau to reduce the possible damage to the chamber without compromising efficiency. Voltage and current outputs of these modules along with those delivered to the PMTs were monitored closely and carefully during data-taking and their nominal setting recorded. The next section will cover how signal from the drift chambers are processed along with the all the associated electronics for this stage. 

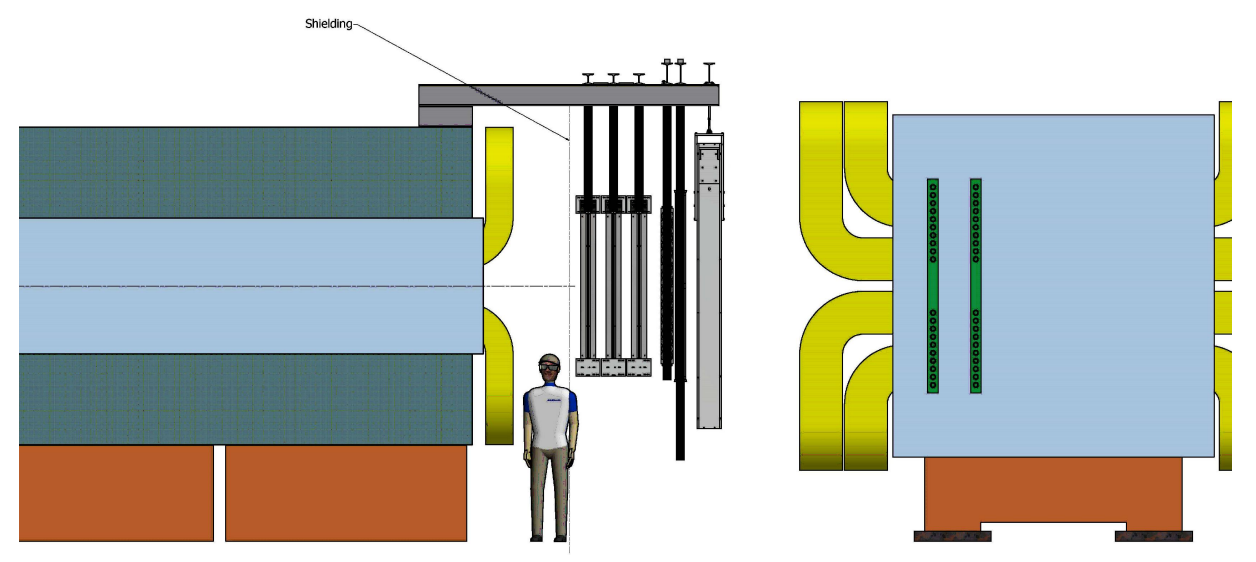

Figure 2.14: Depiction of DC1.1 + DC1.2 configuration.

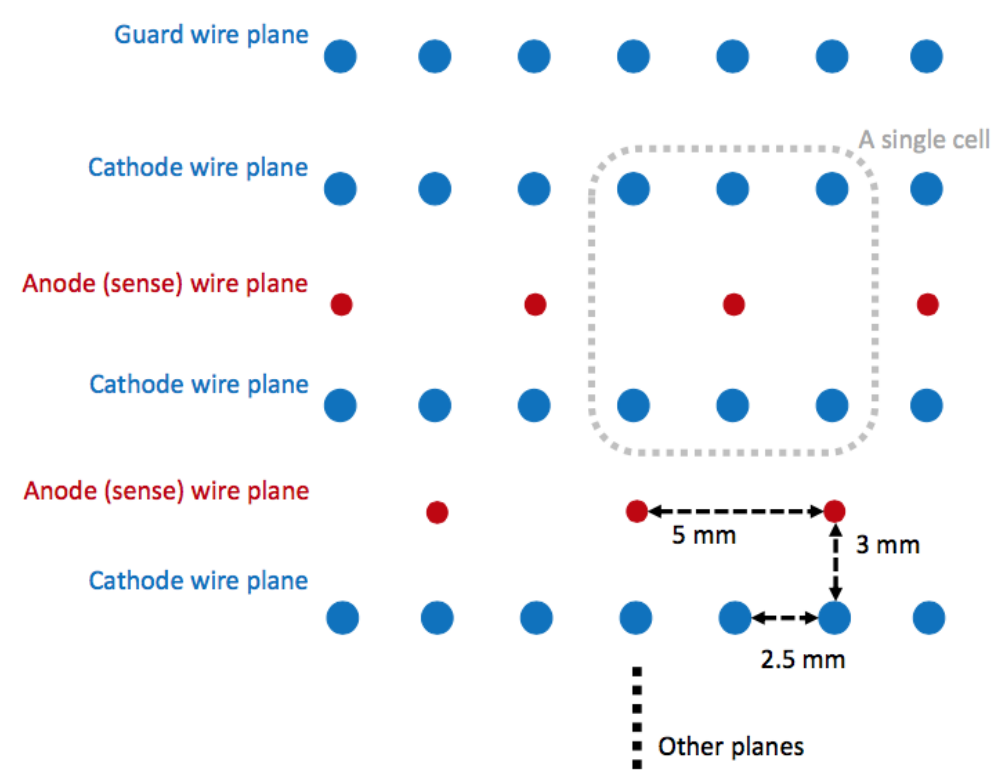

Figure 2.15: Cell structure of DC1.2 


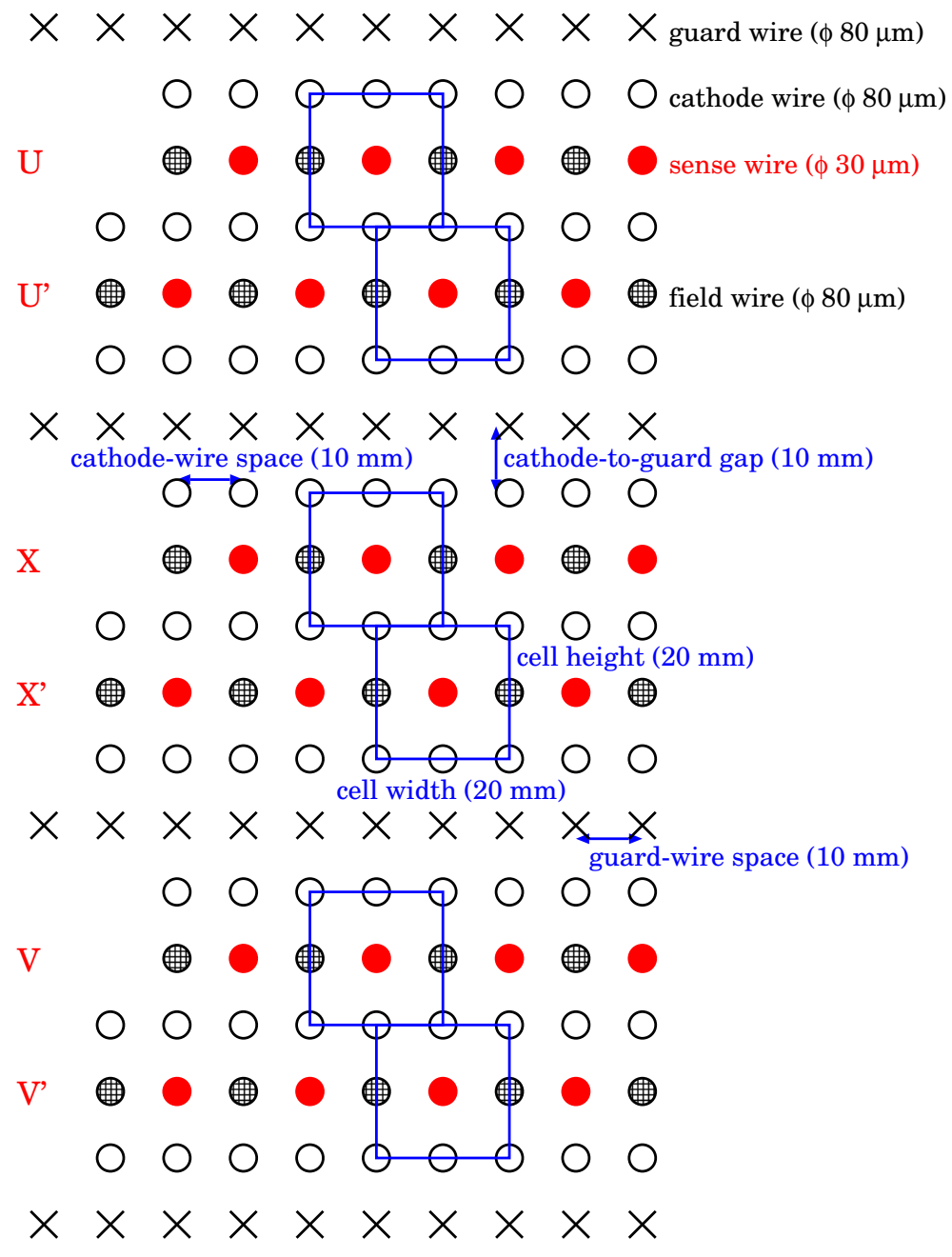

Figure 2.16: Cell structure of DC3p and DC3m 


\subsubsection{Drift chamber readout electronics}

Directly on the drift chambers are ASDQ cards (Amplifier Shaper Discriminator and Charge encoding), which are the first to process the raw analog signals coming from the chambers. A picture of these 8-channel cards is shown in Figure 2.17, including the ribbon cables, the cable supports, and noise-suppressant ferrites. The different elements of the ASDQ cards have the following function:

- Pre-amplifier: amplifies raw signal, converts the charge input into a voltage output and minimizes noise.

- Ion tail cancellation: the signal is amplified even more and its tail is removed.

- Baseline restoration: sets the baseline of the amplified signal to zero.

- Discriminator: discriminates signal below a programmable threshold, while outputting a differential signal for the classifying input. The differential signal is then fed to the "Level Shifter Boards" (LSBs). Their function will be described subsequently.

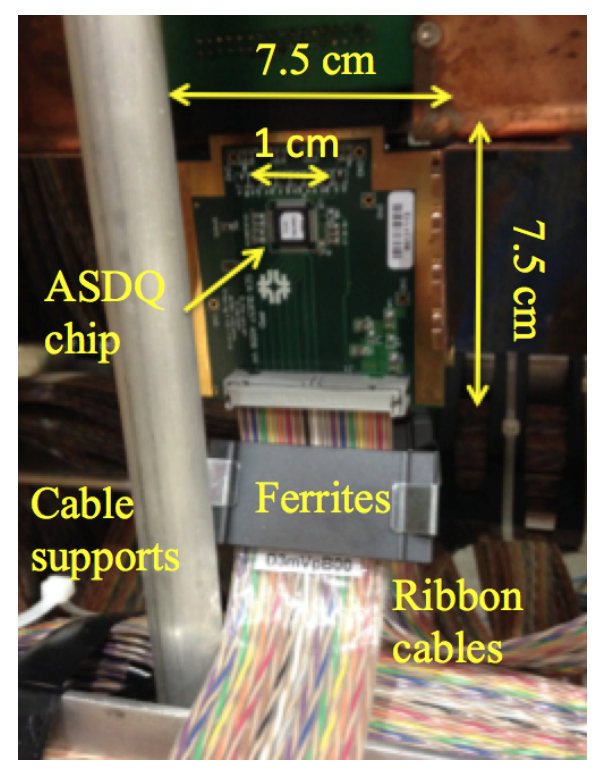

Figure 2.17: Picture of an ASDQ card along with ribbon cables, ferrites (used for noise suppression) and cable supports 
LSBs are specialized boards developed at Fermilab for SeaQuest, which convert the differential signal from the discriminator to standard LVDS (low-voltage differential signal). A master board is selected per drift chamber and controlled using telnet commands. Via these commands, LSBs can also be used to set the ASDQ threshold for noise removal; threshold values are 12 bit (0 - 4096) values that correspond to $0-10 \mathrm{mV}$ for the amplified signal produced at the baseline restoration stage. In addition, LSBs have the option to send test pulses akin to signals from an ASDQ card; a feature that was especially useful during the commissioning period for finding mapping issues in the chain of electronics.

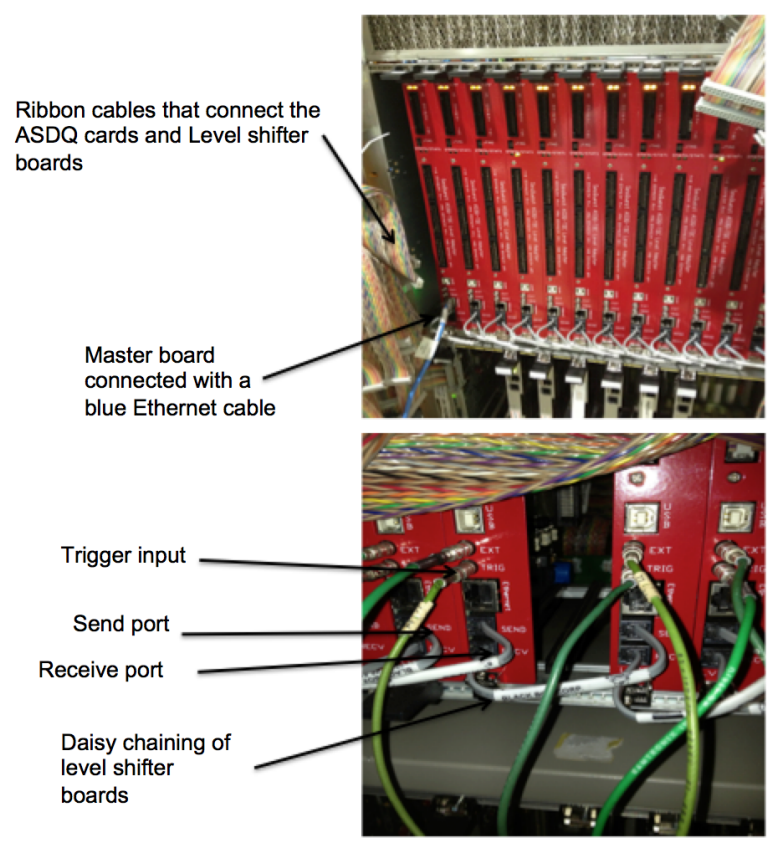

Figure 2.18: Level Shifter Boards 
The LVDS outputs from the drift chambers, hodoscopes and proportional tubes are transmitted to Time to Digital Converter (TDC) modules to be digitized and collected by the DAQ (data acquisition software). The performance of the chambers has been studied using the data collected from Apr. 2014 to Jun. 2015. All the chamber planes have detection efficiency greater than $95 \%$ with $70 \%$ of the planes being nearly $100 \%$ efficient. The single plane efficiency needed to be a minimum of $95 \%$, in order to reach a track reconstruction efficiency of at least $90 \%$ allowing only one inefficient plane at each station. Due to the high multiciplity of background particles, the chambers' key feature (particularly in station 1) are to operate well at high rates of incident particles. The chambers were also designed so that the probability of double hits per wire per event would be small. An average chamber position resolution is typically higher than $400 \mu \mathrm{m}$, corresponding to $\Delta \mathrm{p} / \mathrm{p}(\%)=0.03 \cdot \mathrm{p}(\mathrm{GeV} / \mathrm{c})$ for the momentum resolution. Consequently, the contribution to the total mass resolution from the position resolution is less than $10 \%$ [29].

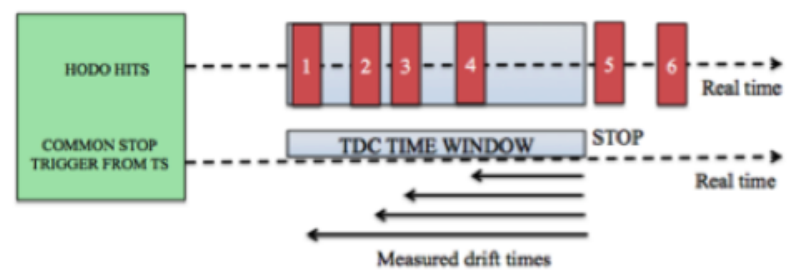

Figure 2.19: Common stop mode of a TDC

\subsection{Proportional tubes}

Proportional tubes are used for muon identification in Station 4 within the SeaQuest spectrometer. Downstream of this station, the particles permeate a $1 \mathrm{~m}$ thick iron wall, serving as a hadron absorber. As they pass through the wall, hadrons that make it through will shower and scatter much more than their leptonic counterparts. A small momentum dependent deflection of a track is thus used as a signature for muon identification. As seen in Figure 2.20, station 4 consists of 4 layers of proportional tube planes, with each plane composed of 9 proportional tube modules. The modules are constructed from 16 proportional tubes (PTs) and these are further divided into 
two staggered sub-planes. The modules grouping tubes measure $2 \mathrm{ft}$ long and are 2 in diameter, with a wall thickness of 1/16 in, and have a central anode made of gold-plated $20 \mu \mathrm{m}$ diameter Tungsten wire (typically set to $1800 \mathrm{~V}$ ). All the PT modules employ the same gas as the drift chambers P08:CF4 (Ar:CH4:CF4 in the ratio 88\%:8\%:4\%) and like the primed-unprimed plane schema of these chambers, this paired-plane configuration of the PTs also aims to resolve the left-right ambiguity of the passing track.

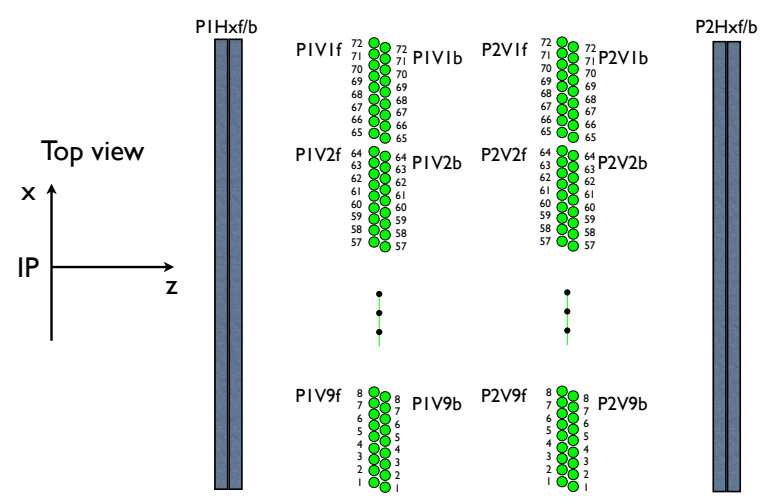

(a) $x z$ view of the proportional tube layout

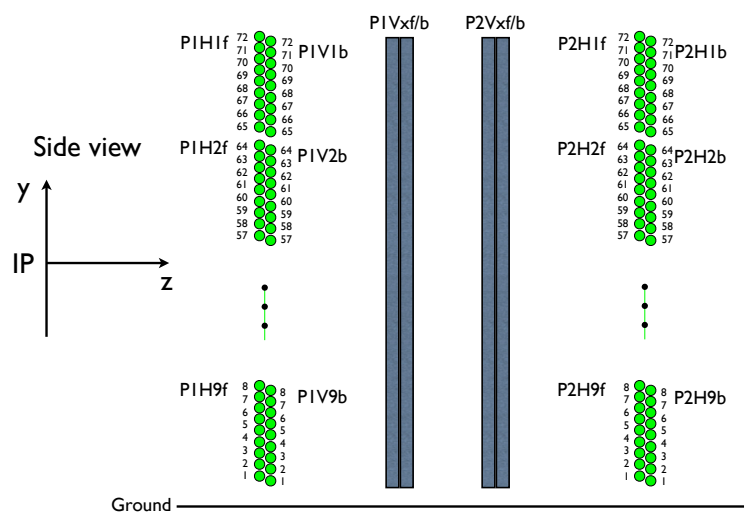

(b) $y z$ view of the proportional tube layout

Figure 2.20: Proportional tubes schema [29]

Analogous to the plane configuration of the hodoscopes, the horizontal PTs in the first and fourth planes measure the y-coordinate of the tracks, while the vertical PTs in the second and third 


\begin{tabular}{|c|c|c|c|}
\hline Plane & $\begin{array}{c}\text { Width } \times \text { Height } \\
(\mathrm{cm} \times \mathrm{cm})\end{array}$ & $\begin{array}{c}\text { Forward sub-plane z-position } \\
(\mathrm{cm})\end{array}$ & $\begin{array}{c}\text { Backward sub-plane } z \text {-position } \\
(\mathrm{cm})\end{array}$ \\
\hline P1V & $368.3 \times 368.3$ & 2175 & 2179 \\
P1H & $368.3 \times 368.3$ & 2099 & 2103 \\
P2V & $368.3 \times 368.3$ & 2367 & 2371 \\
P2H & $368.3 \times 368.3$ & 2389 & 2393 \\
\hline
\end{tabular}

Table 2.5: Proportional tubes settings.

planes measure the x-coordinate. A standard muon track generates hits on two anode wires in each PT plane. From here, the process of position identification parallels that of the drift chambers. N27716 channel Amplifier/Discriminator cards with a common programmable threshold are used to process signals from the groups of PTs. The standard drift time of PTs are $\sim 650 \mathrm{~ns}$ resulting in a hit-rate tolerance of up to $2 \mathrm{MHz}$ for each wire; in general, the hit rate is lower than $1 \mathrm{MHz}$. For muon identification in track reconstruction algorithms, 8 hits from 4 PT modules are used. The position resolution of these detectors was found to be $500 \mu \mathrm{m}$.

\subsection{Trigger}

The SeaQuest trigger employs discriminated signals from the hodoscope counter and is optimized for high DY mass dimuons produced from the targets. In order to keep the triggering rate low enough for minimal DAQ deadtime, it suppresses dimuon events from other target-interacting sources, i.e. quarkonia decays, single muon background resulting from pion decays and cosmic muons, which can flood the trigger. This is due to the fact that the trigger does not differentiate between where the track originated; an interplay of similar track kinematics is enough to satisfy its dimuon-selecting criteria. The trigger system design attempts to address this via two core modules: a NIM-based trigger and an FPGA-based trigger.

The NIM-based trigger uses the Nuclear Instrumentation Module (NIM) standard for trigger construction, which defines mechanical and electrical specifications for nuclear/particle physics experiments. There are two types of NIM-triggers used under standard data taking conditions: NIM1 and NIM3. The former triggers on the coincidence of signals from the top-half or the bottom half 
y-measuring hodoscopes of all stations $(\mathrm{H} 1 \mathrm{~T}+\mathrm{H} 2 \mathrm{~T}+\mathrm{H} 3 \mathrm{~T}+\mathrm{H} 4 \mathrm{~T}$ or $\mathrm{H} 1 \mathrm{~B}+\mathrm{H} 2 \mathrm{~B}+\mathrm{H} 3 \mathrm{~B}+\mathrm{H} 4 \mathrm{~B})$. It does not distinguish between the charge of the muons and can therefore be utilized to analyze spectrometer issues including, hodoscope paddle efficiencies, timing issues and cosmic muon scans. The latter is a special pseudo-random trigger formed by the coincidence of the RF signal from the Fermilab Accelerator Division and a $7.5 \mathrm{kHz}$ pulse produced by a gate generator. When there is an overlap between the two signals, the NIM-3 trigger records events from the "randomly" selected RF bucket, information that is collectively useful for comprehensive background studies. For example, NIM-3 events are embedded in the "clean"-event Monte Carlo productions in order to simulate and study the effects of background on track reconstruction efficiencies and other experimental/analysis parameters.

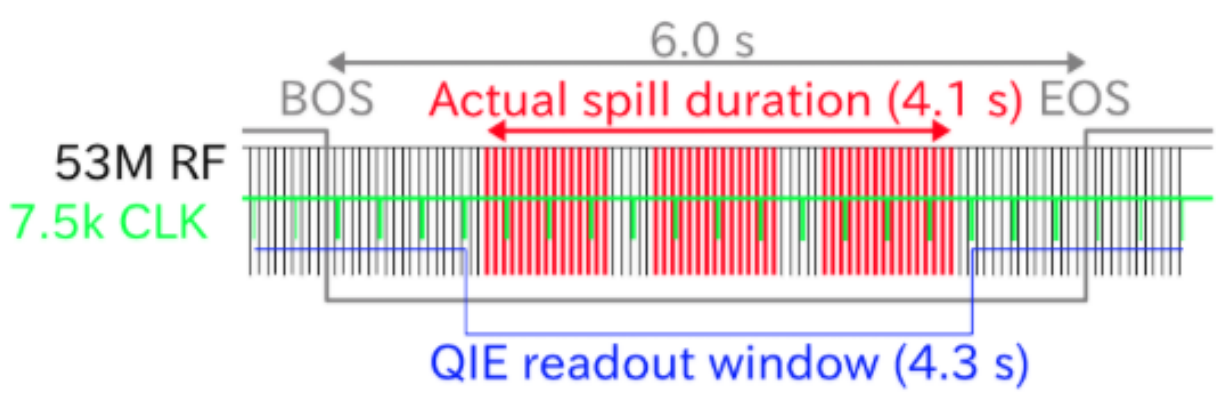

Figure 2.21: Function structure of the NIM3 pseudo-random trigger.

The second core module, the FPGA-based trigger, uses one Altera EPIC20F400C6 Field Programmable Gate Array (FPGA) integrated with nine CAEN V1495 VME modules. In total, there are five FPGA triggers that were used on SeaQuest (FPGA 1 - 5), each comprised of three subparts or "levels" of the V1945 VME module (Level-0, Level-1, Level-2). The inputs received by the trigger are discriminated signals from four hodoscope planes. These signals are classified into four different quadrants, corresponding to the two halves of the $\mathrm{X}$ and Y-planes, and are then processed by a Level-0 VME module. The module is set to either "Pulser" or "Production" mode, depending on what it is being used for. The "Pulser" mode is useful to study the status of the Level-1 and Level-2 triggers. In this mode, text files with preset hit patterns as output are used as a diagnostic tool to detect loss of signal and gauge other functionalities of these triggers. During data taking, 


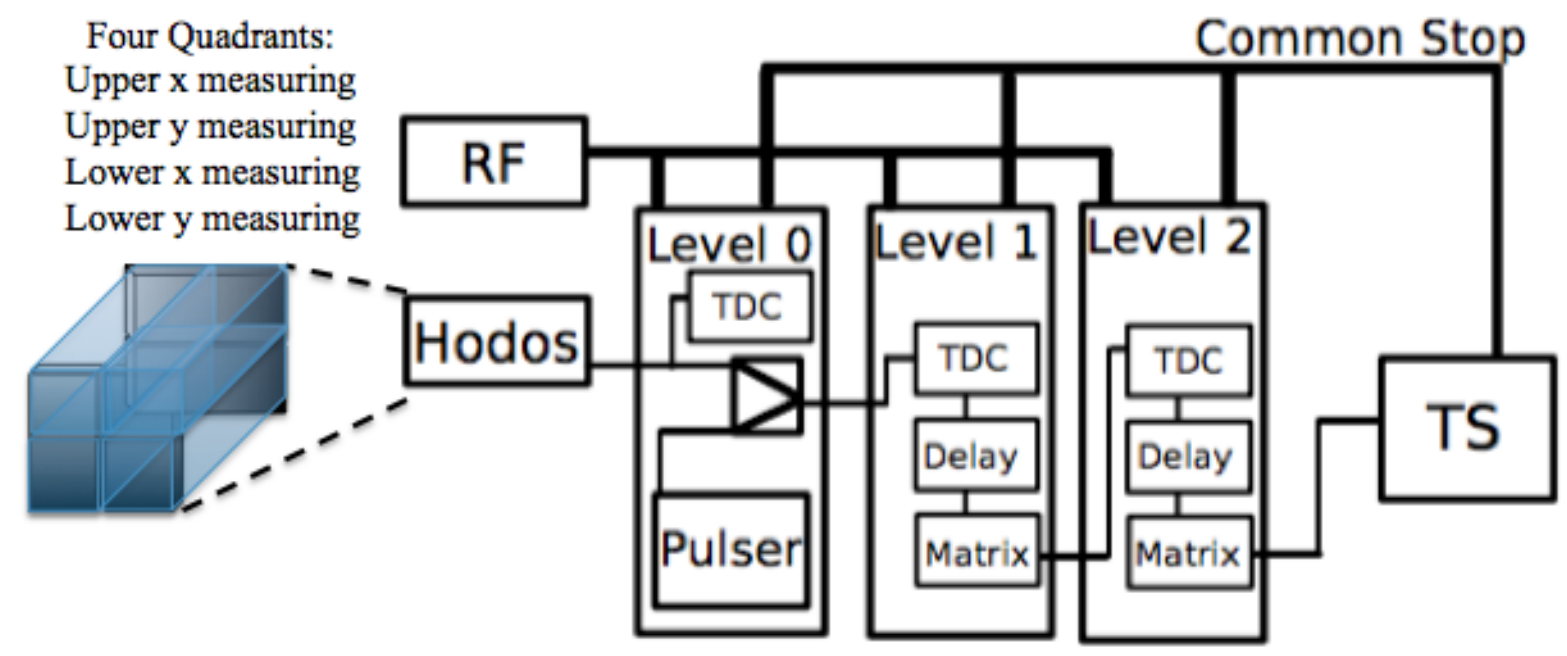

Figure 2.22: SeaQuest trigger diagram [32].

the Level-0 module is set to the "Production" mode and the input signals are sent straight to the Level-1 modules.

Each of the four V1495 modules making up the Level-1 trigger receive input from one Level-0 board. The Level-1 trigger determination itself only uses the X-plane Level-0 module information. The function of the trigger at this stage is to find 4-hit tracks of candidate muons and it accomplishes this by matching up hits on the hodoscope planes to a trigger road, a compilation of prescribed hit patterns; a particular set of trigger roads is referred to as a "Roadset". The Roadset was then iteratively resolved from data, after some preliminary estimations from Monte Carlo simulations looking at detector location hits both for signal and background along with reconstruction efficiencies and parameters. Each Roadset adjustment was awarded an ID index- Roadset 57 was the first one to be used for data analysis.

After the trigger road pattern matching is complete and prior to sending information to the Level-2 trigger, a sequence of bits binned by charge and average transverse (X-direction) momentum $p_{x}$ are produced. The Level-2 trigger is a track correlator which employs only one V1495 module. It takes the track candidates identified in Level-1, pairs them in all possible ways and then cross-checks the validity of the pairs by implementing lookup tables of roads as a trigger matrix. 
As previously mentioned, five unique Level-2 trigger requirements were used at SeaQuest for different data sifting purposes and their specifications are defined on Table 2.6. FPGA-1 triggers on two oppositely charged tracks with one in each half of the detector and represents SeaQuest's main physics trigger. Trigger information from each of these five Level- 2 triggers is then delivered to the Trigger Supervisor (TS), a VME module discussed in more detail in the subsequent section.

\begin{tabular}{lclcl}
\hline Name & Side & Track charge & Prescale factor & Remarks \\
\hline NIM 1 & Y coincidence & $+/-$ & - & - \\
NIM 2 & X coincidence & $+/-$ & - & - \\
NIM 3 & Random RF & $+/-$ & - & RF clock $+7.5 \mathrm{kHz}$ clock \\
FPGA 1 & TB/BT & $+-/-+$ & 1 & Main physics trigger \\
FPGA 2 & TT/BB & $+-/-+$ & 1000 & Same-side trigger \\
FPGA 3 & TB/BT & $++/--$ & 123 & Like-charge trigger \\
FPGA 4 & T/B & $+/-$ & 25461 & All singles trigger \\
FPGA 5 & T/B & $+/-$ & 2427 & High- $p_{T}$ singles trigger \\
\hline
\end{tabular}

Table 2.6: Settings for the different SeaQuest triggers. For FPGA 5, $p_{x}>3 \mathrm{GeV} / \mathrm{c}$ is an additional requisite [29].

\subsection{Data acquisition (DAQ)}

Given the different timing and bandwidth needs as the data is acquired and processed, the data acquisition (DAQ) system of SeaQuest is divided into three sub-systems referred to as "Main DAQ", "ScalerDAQ", and "Beam DAQ", each serving a particular function. Details on each of these systems will be discussed in this section.

\subsubsection{MainDAQ}

The MainDAQ communicates directly with the FPGA trigger matrix or the NIM triggers and it's primary function is to record the event-by-event main detector information and trigger timing. The body of the MainDAQ is comprised of $13 \mathrm{VME}$ crates and the TS, which receives trigger signals and distributes them to the other crates. As seen in Figure 2.23, each of the 13 crates have a Trigger Interface (TI), a main VME processor or ROC (Read Out Controller) and $\sim 6$ to 7 TDCs 
for detector signal acceptance. The TS has 12 trigger slots: four NIM-based, five FPGA-based and the rest for end-of-spill (EOS) and beginning-of-spill (BOS) signal.

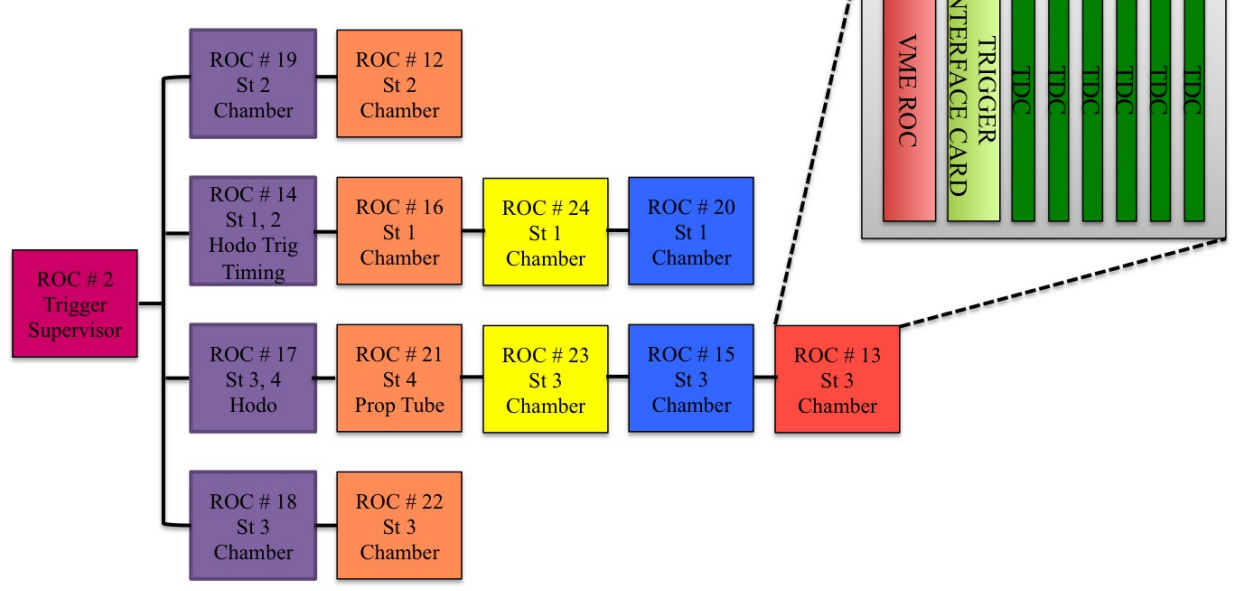

Figure 2.23: Trigger distribution schema. Readout is asynchronous [4].

The data flow through this system can be seen in Figure 2.24. Eight of the input channels from the TS can be pre-scale by a factor. In doing so, only one out of a number of events received, defined by the pre-scaled factor for that trigger, would be recognized by the TS. The FPGA trigger rates are scaled down to less than $\sim 10 \%$ of the FPGA-1 trigger rate (see Table 2.6 for FPGA factors) so that there is sufficient bandwidth for the main physics trigger. Details of the workflow are as follows:

- The Trigger Supervisor (TS) receives the trigger from the V1495 Level-2 or NIM modules and TS is set to busy.

- After being delayed by $32 \mu \mathrm{s}$, the TS outputs all accepted triggers to the TI cards (a process referred to as copy-in-progress time). 


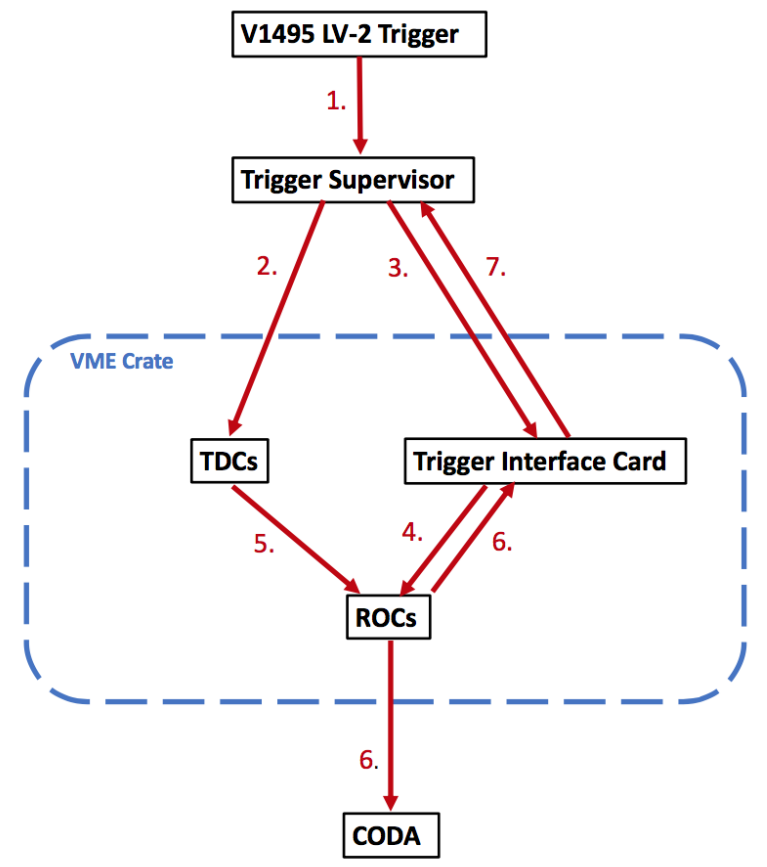

Figure 2.24: Workflow of the MainDAQ [18]

- After another $10 \mu$ s have passed, each TI sends an order to the ROC so that the TDCs are readout.

- The TDCs send hit information to the ROC through the VME backplane (lasting about 100 $\mu \mathrm{s})$.

- The ROC informs the TI that the reading process is finalized and the collected data is sent to CODA through a private network.

- Once the TS receives an acknowledgement signal (ACK) from all the 13 ROCs, the VME readout is done, the TS is reset (busy signal clear) and ready to take information on the next trigger.

The process is iterated over until a run is finished (here, run is an individual 1 hour-long period of data taking). The readout time was reduced from $\approx 150 \mu$ s to $\approx 30 \mu \mathrm{s}$, towards the end of 2016 , when data began to be stored locally in the TDC modules during a spill and sent between spills through the VME backplanes. 


\subsubsection{ScalerDAQ}

The function of the Scaler DAQ is to collect data from the beam, detectors and trigger for monitoring and diagnostic purposes. It is controlled by a stand-alone CODA system running on its own computer and thus is completely independent of the Main DAQ. The system uses one VME crate with a ROC CPU board and four scaler cards. One of the channels counts the coincidence of the 7.5 $\mathrm{kHz}$ gate generator and the beam spill signal using the response of two unrelated hodoscopes. This information is used to calculate the duty factor, an important beam quality measurement defined in Section 2.9.3. The other three scalers are triggered by BOS and EOS and record trigger, inhibit and hodoscope counts per spill, including the rates of the hodoscope arrays and when MainDAQ trigger is satisfied.

\subsubsection{BeamDAQ}

The primary function of the BeamDAQ is to record RF intensity information, i.e. the $53 \mathrm{MHz}$ structure of the beam on a bucket-by-bucket basis. The BeamDAQ begins the readout at the arrival of EOS signal and all the data is output to ASCII files. Using information from the Čerenkov detector in the proton beam, it also calculates the duty factor, $D F$, defined as

$$
D F=\frac{<I>^{2}}{<I^{2}>}
$$

where $I$ is the bucket-by-bucket sum of beam intensity and $I^{2}$ is the sum of the bucket-intensity squared for every spill. As the $D F$ gets closer to one, the beam quality is most stable in intensity throughout all the buckets in the spill. This value is used for beam fine-tuning by the Accelerator Division. The control and readout of the QIE module is carried out by the custom DAQ program that communicates with the QIE through a $100 \mathrm{Mbps}$ Ethernet interface. In addition to the DF intensity parameters, three other types of data are recorded by the QIE board during the spill: (1) The intensity of each individual RF bucket. (2) The number of protons inhibited due to the high instantaneous intensity. (3) The number of protons missed as the BeamDAQ was busy during 
readout.

\subsubsection{Slow controls}

The slow control system uses scripts for the purpose of synchronizing the DAQ data stream, retrieving and storing per-spill frequency data and monitoring different process variables (target-rotation pattern, the temperature and pressure of the cryogenics, etc.) along with the general status of the experiment. The EPICS system (Experimental Physics and Industrial Control System) is employed by the slow control scripts for data relaying over the network.

Several "live" checks are also executed by the monitoring scripts. These include monitoring the state of the targets, the available disk space, the status of the DAQ system and is the data is updating regularly [18].

\subsection{Data decoding and storing}

A one hour run is analogous to $\sim 1$ GB of raw data accrued by the Main DAQ. The Main-DAQ CODA file and the data produced by other DAQ subsystems are warehoused on the SeaQuest servers and backed up by the tape storage service managed by the Fermilab Computing Division. These raw data files are processed by the "decoder" program and the decoded data is extracted into the SeaQuest MySQL database. A unique MySQL schema is produced for each run where the data is stored and information is classified into tables. The data is duplicated across multiple MySQL servers and is easily accessed for analysis and reference purposes [18]. 


\section{Chapter 3 Data analysis}

This data analysis seeks to study different types of CNM effects by isolating signal in the form of $J / \psi$ and Drell-Yan target events from background processes coming from upstream the beam-line and the iron dump. The specifics of the analysis will be covered in this section.

\subsection{Data profiling}

The data sets used in this analysis were Roadset (RS) 57, 59, 62, 67 and 70, where "Roadset" refers to a set of trigger roads used during that data taking period. Settings and specifications for these and other roadsets can be found in Table 3.1 and 3.2. Due to various issues related to trigger timing shifts, missing or bad QIE values, FMAG settings among others, some spills were excluded from the analysis. As was noted below, there was a vertical offset of the beam introduced between Roadsets 59 and 62 and the magnetic field was switched between Roadsets 62 and 67. These changes were represented in Monte Carlo simulations, track reconstruction and analysis cuts.

\begin{tabular}{|c|c|}
\hline Roadset & Description \\
\hline \hline 49 & Initial GMC roads, hot roads removed \\
\hline 57 & New GMC roads, improved cuts, hot roads removed \\
\hline 59 & Added a few dark photon roads \\
\hline 61 & fastMC half FMAG-field roads \\
\hline 62 & Recompiled Roadset \# 57 with RF-Clocking \\
\hline 67 & Charge symmetry enforced, hot roads removed (mag field flip) \\
\hline 70 & Dark photon roads added and some hot roads removed from 67 \\
\hline 78 & $\begin{array}{c}\text { GMC roads to include new DC1.2 acceptance, proton straight-through roads } \\
\text { and dark photon roads added, some hot roads removed from 67 }\end{array}$ \\
\hline
\end{tabular}

Table 3.1: Specifications of roadsets. 


\begin{tabular}{|c|c|c|c|c|}
\hline Roadset & run range & spill range & beam offset & B orientation \\
\hline 57 & $8912-10420$ & $310955-370099$ & $0.4 \mathrm{~cm}$ & $\mathrm{~B}+$ \\
\hline 59 & $10421-10912$ & $370110-388469$ & $0.4 \mathrm{~cm}$ & $\mathrm{~B}+$ \\
\hline 62 & $11075-12435$ & $409547-482571$ & $1.6 \mathrm{~cm}$ & $\mathrm{~B}+$ \\
\hline 67 & $12525-15789$ & $484746-676223$ & $1.6 \mathrm{~cm}$ & $\mathrm{~B}-$ \\
\hline 70 & $15793-16076$ & $676498-696454$ & $1.6 \mathrm{~cm}$ & $\mathrm{~B}-$ \\
\hline
\end{tabular}

Table 3.2: "Good" run and spill ranges.

\subsection{Reconstructing dimuons}

The primary challenge with the reconstruction of viable tracks at SeaQuest is the multiple scattering of tracks as they traverse the FMAG/beam dump iron wall. A robust track reconstruction program referred to as "kTracker" is employed by SeaQuest to address this and other track reconstruction requisites. This program was principally developed by Kun Liu, a collaborator from Los Alamos National Laboratory and uses a Kalman-Filter method for dimuon vertex reconstruction. The process of reconstruction can be outlined into three stages: pre-tracking analysis, track reconstruction, and vertex finding. The workflow of kTracker is shown in Figure 3.1 and its principles will be detailed in this section $[33,34]$.

\subsubsection{Pre-tracking data trimming methods}

A preliminary trimming of noise hits carrying certain unifying characteristics is performed prior to running the tracker. This enhances its performance and reduces the tracking process time. These actions are applied to all detector hits:

- Rejecting out-of-time hits: Used to remove random noise and signals from undesired sources, i.e. cosmic rays. Purges hits with TDC time falling out of a set TDC time window.

- After-pulse removal: Used to remove hits that could come from the echo of signals in the same channel. This is done by only accepting the first pulse of each channel in an event.

Trimming is also applied to drift chamber signals in "hit clusters", which refer to groups of neighboring fired wires. These are classified into three categories depending on what led to their 


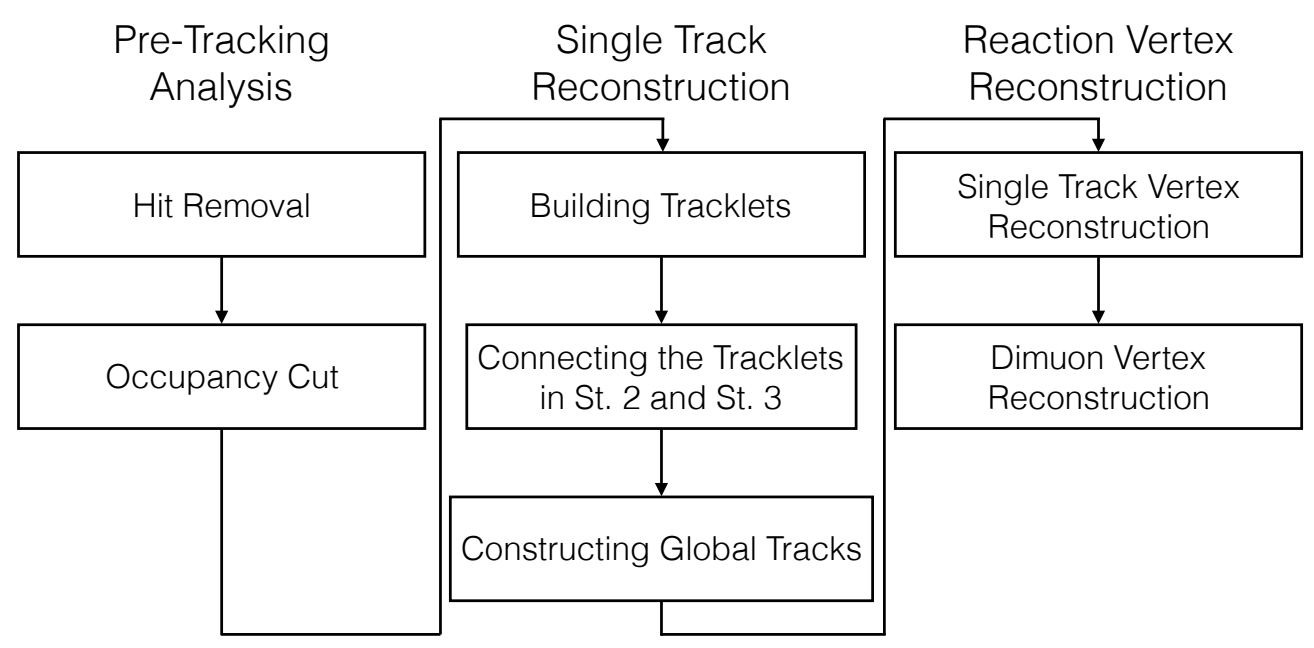

Figure 3.1: Flowchart for kTracker from [4].

creation. Sources of hit clusters and how they are identified include the following:

- $\delta$ rays: These are high-energy electrons that may originate from close to head-on collisions in the passage of a primary ionizing particle and can produce secondary ionizations. The signature of a $\delta$ ray moving along the $\mathrm{X}-\mathrm{Y}$ plane is a cluster consisting of more than two continuous hits with large average TDC-time differences (10 ns or larger). The two hit edges are stored and the middle hits are discarded in order to maintain viable hits that could have been generated by a muon track.

- "cell-edge" hits: A track passing near the center of two adjacent wires may induce ionizations that fire both wires. This results in the respective hits having long drift distances (about half of the cell width). The hit with the longest drift distance is therefore removed.

- electronic noise: If two or more hits on neighboring wires have average TDC time differences less than $10 \mathrm{~ns}$, they are discarded as electronic noise.

Next in the track trimming process is constraining the amount of hits in a detector. This is done via the application of "multiplicity cuts" and it's primarily used to remove events with high multiplicities (or occupancies) that extend the tracking time and reduce tracker efficiencies. These occupancy limits are summarized on Table 3.3. 


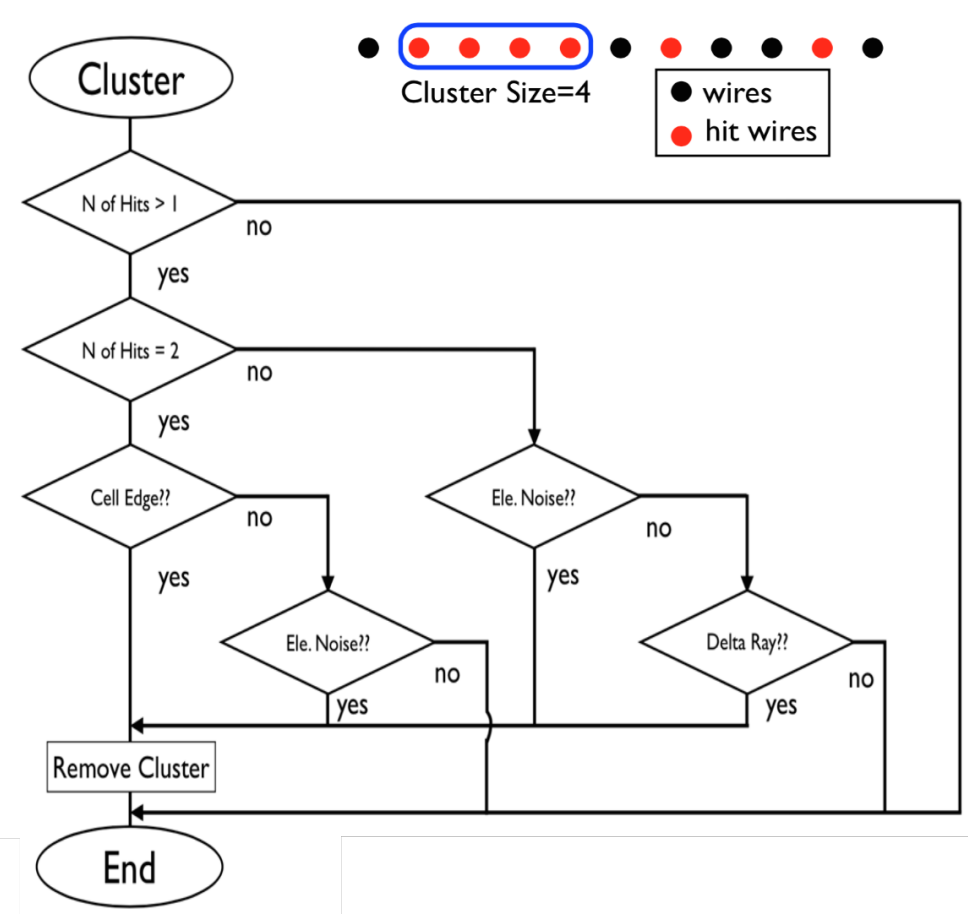

Figure 3.2: Cluster removal flowchart [35]

In conjunction to these trimming methods, a trigger analysis is performed on the raw data. Unless a set of hits correspond to an enabled road, i.e. a set of four x-plane hodoscope in-time hits match any road candidates, the hits are discarded. Furthermore, if an event is found to have five or more possible $\mu^{+}$or $\mu^{-}$roads, this event is removed as an additional effort to preserve optimal tracking time and efficiency. Finally, proportional tubes are also employed at this stage for "tracklet" building. These are local tracks inside the detector used as seeds in reconstructing global tracks for future muon identification. [36].

\subsubsection{Reconstructing tracklets}

The first phase in single track reconstruction involves an analysis of tracklets within individual chambers. The primary detectors employed here are DC2 and DC3, where tracks traverse from one chamber to the other in a line due to the absence of a magnetic field. Hits in these chambers are comparatively less noisy than in station 1 detectors facilitating this preliminary tracking analysis. The analysis consists of looking for tracklets as hit triplets of the three different views of a chamber. 


\begin{tabular}{|c|c|}
\hline Detector & Occupancy limit \\
\hline DC1.1 & 350 \\
\hline DC1.2 & 350 \\
\hline DC2 & 170 \\
\hline DC3m & 140 \\
\hline DC3p & 140 \\
\hline H1T + H1B & 15 \\
\hline H2T + H2B & 10 \\
\hline H3T + H3B & 10 \\
\hline H4T + H4B & 10 \\
\hline Proportional tubes & 300 \\
\hline
\end{tabular}

Table 3.3: Cuts on the number of detector hits [4] [18].

Figure 3.3 shows a visual representation of this process consisting of the following steps:

- Step 1: Tracker identifies adjacent hit pairs in the primed and unprimed planes in the X-view.

- Step 2: For a given $\mathrm{X}$ hit, tracker then combines all the $\mathrm{U}$ view hits in the allowed window. This area is defined by geometry and maximum track slope and is $\sim 20 \mathrm{~cm}$.

- Step 3: Next, for a given $X-U$ doublet, the tracker can constrain the possible V view hits to a smaller window $(\sim 5 \mathrm{~cm})$.

- Step 4: The tracker then fits the triplet without drift distance, assuming the spatial resolution of wires is determined by the wire spacing (multiple triplets are allowed to use the same hits here).

- Step 5: Triplets are discarded if:

- they do not roughly point to a fired hodoscope paddle in a neighboring $\mathrm{x}$-hodoscope station.

- they have less than 4 associated hits, or the hits from a specific view are missing.

- they do not point back to target (loose cut).

- their total fitting $\chi^{2}$ is greater than 15. 

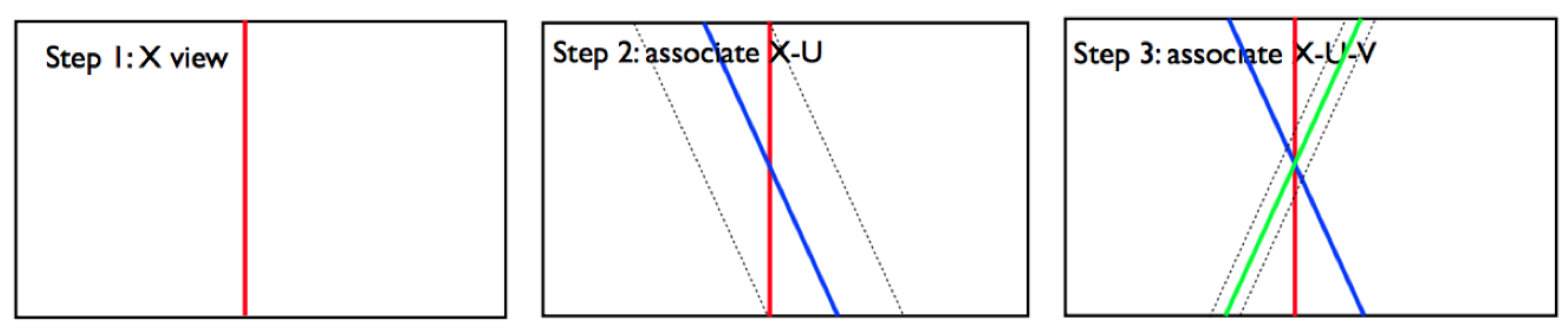

Figure 3.3: Reconstructing drift chamber tracklets [36].

The identified triplets then go through a recursive matching process where all possible tracklet combinations between station 2 and 3 are made. Bad tracklet combinations are eliminated via quality cuts and the surviving ones are sent through a $\chi^{2}$-based fitter to form partial tracks between these chambers. Only the partial track with a smaller $\chi^{2}$ is kept when two partial tracks have more than $1 / 3$ of the hits in common. If the resulting partial track is not pointing towards the target or to the fired paddles in the hodoscopes of the last three stations, the tracklet combo is eliminated. In addition, a muon-identification cut is also used requiring that the projected partial track has at least one matched hit at the Station 4 proportional tubes, factoring in a minor deflection caused by the interaction with the iron wall.

The partial-track candidates from station-2-to-3 tracklet combos are then projected to station 1 and combined with a suitable station- 1 tracklet forming a "global track". This is done via the "sagitta" method, which provides a search window for building station-1 tracklets. The sagitta represents the distance between the track and the line connecting a station- 3 triplet and $\mathrm{X}=\mathrm{Y}=\mathrm{Z}=0$. The sagitta ratio is momentum independent and defined as the ratio of the distance "s1" and "s2", as seen in Figure 3.4. Using Monte Carlo simulations, this value was calculated to be $1.77 \pm 0.055$. This value is subsequently used to determine a window width for station- 1 hits of $\pm 5 \mathrm{~cm}$, within which the station-1 triplet will be built and connected to its respective station-2-to-3 partial track. 

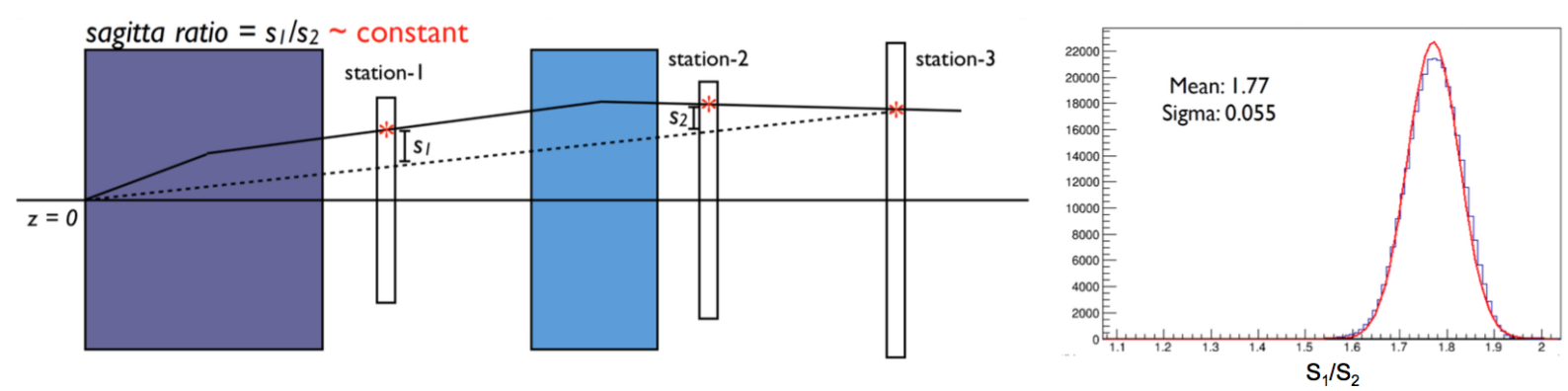

Figure 3.4: Track projection via the sagitta method from [36].

\subsubsection{Reconstructing global tracks}

Global track candidates formed via the saggitta method first undergo an iterative cleanse of bad hits. Track fits are reviewed. Hits with the greatest residual are discarded, along with any hit residuals that are greater than three times the chamber resolution $(\sim 7 \mathrm{~mm})$. This process is repeated until all the hits of a global track candidate have residuals below $7 \mathrm{~mm}$. Surviving tracks must:

- have at least one hit in each view and four hits in each station.

- point to fired X-hodoscope paddles in all stations.

- have momentums greater than $5 \mathrm{GeV}$ and less than $100 \mathrm{GeV}$.

- not be deflected by the absorber wall more than anticipated, given their momentum.

A Kalman Filter (KM) algorithm for fine fitting is utilized on this new batch of global tracks [34]. This recursive program is used to find the best estimation of an unknown state vector given some initial conditions. The algorithm works in a two-step process: In the predictive stage, the KM produces estimates of the current state variables with their corresponding uncertainties, attempting to successfully handle the error due to noisy data and other random external factors. In the measurement output stage, the algorithm yields an estimate of the state vector in the form of an average of the system's predicted state plus the new measurement with a weighted average. The latter has a better estimated uncertainty than either the predicted or the measured state by themselves. This procedure is repeated many times, with the new estimate and its weight guiding the prediction for 


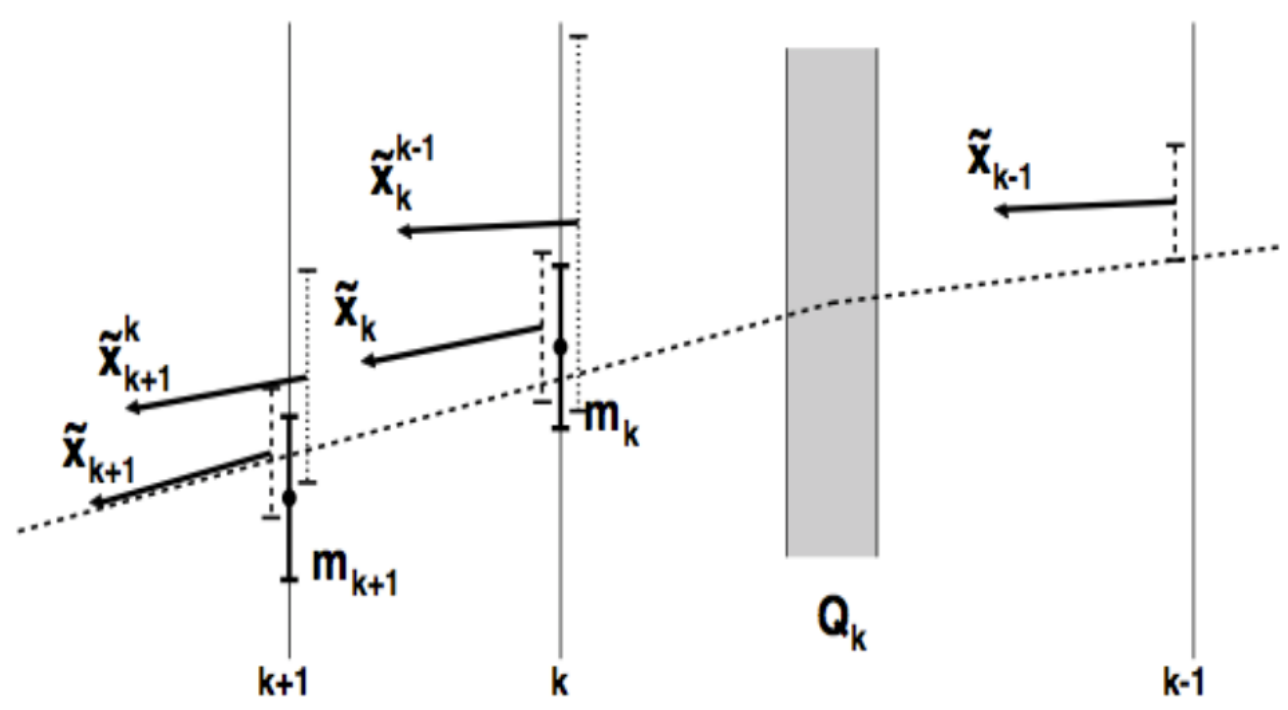

Figure 3.5: Geometric depiction of the Kalman filter process [36].

the next iteration. The KM only requires the last best guess rather than entire history of a system's state to produce a new state. The workflow of the Kalman filter process is shown in 3.6 [18].

For SeaQuest, the state vector inputted into the KM algorithm is defined by the spatial coordinates of a muon and its three-momentum. Muonic parameters vary as the state is evolved, i.e. as the muon traverses through the spectrometer. The measured initial conditions inputted into the KM include the hit position for each triggered detector plane and the respective uncertainty related to their resolution. The dynamic evolution linking states is produced with the GEANT4 software package. This platform simulates muonic interactions through the SeaQuest spectrometer, propagating the state from downstream to upstream of the spectrometer. This direction of state propagation allows for better defined initial state variables and propagation due to the lower background atmosphere in the downstream region of the spectrometer, enhancing the quality of convergence in the KM estimation. 


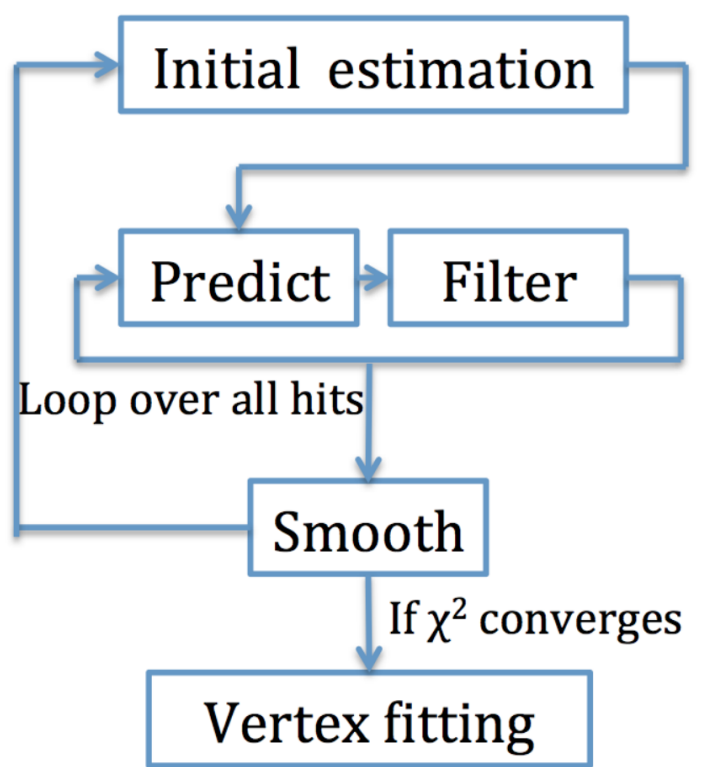

Figure 3.6: Workflow of the Kalman filter process [36].

\subsubsection{Vertex reconstruction}

As the path of each single muon is reconstructed back from the downstream stations, kTracker handles the muon traversing through the FMAG with particular care. First off, it is divided into 100 slices (5 cm steps) in the z-direction allowing the energy loss of the muon to be discretely accounted for. In addition, a traverse momentum kick $(2.909 \mathrm{GeV} / \mathrm{c})$ is applied at the center of every slice. Each step is divided into two half-steps as follows:

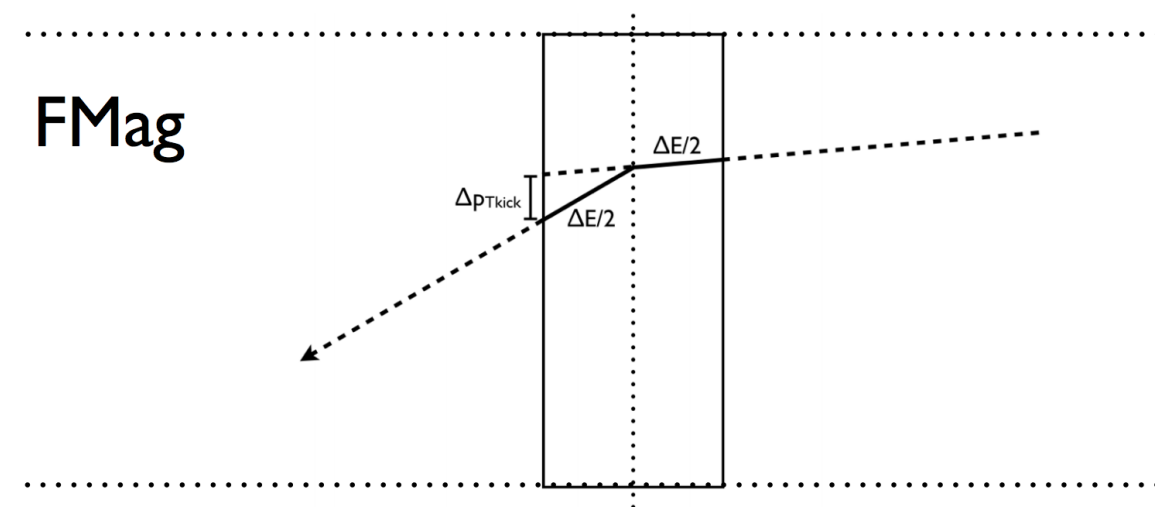

Figure 3.7: Procedure for muon tracks through a slice of FMAG from [36] 
- An energy loss (energy gain, due to backward propagation in the reconstruction) is applied at the first half-step (corrected for the actual travel length).

- $\mathrm{A} p_{T}$ kick at the center of the slice.

- An energy loss is applied again at the second half-step.

The process is continued until the muon crosses all of FMAG and their tracks are extrapolated to the target region. The position with the closest approach to the beam line is classified as the vertex of the track.

\subsubsection{Dimuon vertex reconstruction}

An extended version of the KM process, pioneered by Gorbunov and Kisel, is used during the vertex reconstruction phase. In this case, the associated state vector is the dimuon vertex position. The first estimation of the vertex position corresponds to two $\mathrm{Z}$-positions (with $\mathrm{X}=\mathrm{Y}=0$ ): the average z-position of the two single muon vertices or the distance of closest approach. The KM method updates the dimuon vertex position iteratively using these original inputs and the results are required to fall within a viable Z-position as they converge. If they do not, the procedure is repeated now using with the new vertex position as the original input. The result with the best vertex-fitting $\chi^{2}$ is stored [33].

It is important to note that there are other effects aside from the energy loss within FMAG, i.e. multiple scattering, that affect the vertex reconstruction. kTracker addresses this by defining an auxiliary factor, $\mathrm{Z}_{\text {opt }}$, which aims to correct the mass and vertex position based on Monte Carlo simulations. If the dimuon is thought to come from the target with the condition on both muons as:

$$
\chi_{\text {target }}^{2}<1.5 \chi_{\text {dump }}^{2}
$$

then the vertex optimization condition is applied to the dimuon. The optimization formula for $\mathrm{Z}_{\text {opt }}$ 


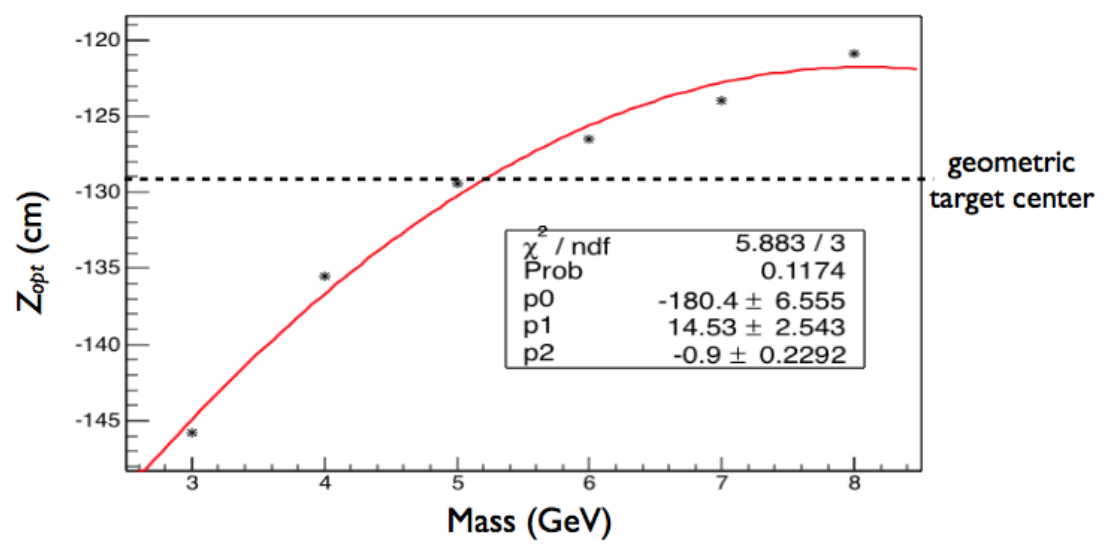

Figure 3.8: Vertex position for a set Z-position (dotted line) and mass [36].

is given by:

$$
-305.465+104.731 m-24.3589 m^{2}+2.5564 m^{3}-0.0978876 m^{4}
$$

As can be seen in Figure 3.8, the optimization puts the dimuon vertex downstream for low mass and upstream for high mass for target likely dimuons. A different formula is used for events believed to come from the dump. [36].

\subsection{Monte Carlo simulations}

The Monte Carlo Simulation program used at SeaQuest aims to characterize and predict the outcome of the experiment. Two different Monte Carlo simulation programs were employed: Fast Monte Carlo (FastMC) and GEANT Monte Carlo (GMC). The FastMC program, also used in the E866 experiment, was used to generate quick but not the most sophisticated analysis results. The GMC program is a GEANT4 based Monte Carlo and a powerful tool to examine the magnet's fringe field, where the fringe field is mapped by field probes in the hall and then the map implemented in GEANT, as well as the dynamics and effects of particles passing through the spectrometer. Most specifically, it can be used to study reconstruction parameters related to tracking, detector hit patterns, analysis cuts, and spectrometer efficiencies. GMC has four types of genera- 
tors: dimuon generator, single muon generator, gun generator and $\eta$ generator and two main $\mathrm{MC}$ simulation formats: "clean" and "messy" MC simulations.

\subsubsection{Dimuon generator}

This GMC produces dimuons in selected kinematic variables from three different sources: DrellYan, $J / \psi$ or $\psi^{\prime}$ decays. Settings and variable ranges for $J / \psi$ or $\psi^{\prime}$ dimuons are as follows:

- $J / \psi:$ mass $=3.097 \mathrm{GeV}$.

- $\psi^{\prime}:$ mass $=3.686 \mathrm{GeV}$.

- $p_{T}^{2}>0$.

- Specific $p_{T}$ distributions use are found in [37].

Setttings and variable ranges for Drell-Yan dimuons are as follows:

- $2 \mathrm{GeV} / c^{2}<\operatorname{mass}<10 \mathrm{GeV} / c^{2}$.

- $-1<x_{F}<1$.

- $0<x_{B}<1$.

- $0<x_{T}<1$.

- $p_{T}^{2}>0$.

- $p_{T}$ distributions are parameterized for an $800 \mathrm{GeV}$ beam and re-weighted for a $120 \mathrm{GeV}$ beam. See [38] for specifics.

- $4 \pi$ acceptance for the spectrometer.

- Dimuons are weighted according to the DY cross-section multiplied by the K-Factor (sigweight).

- Different packages of PDFs where used depending on preference. 


\subsubsection{Background generators}

- Single muon generator: Not used in any robust physics analysis at SeaQuest.

- Gun generator: This generator produces different primary and secondary particles aside from muons, i.e. $\pi, K$, that could come from a proton interacting in target region. On average, the process of simulating and reconstructing tracks from so many protons $\left(10^{7}\right)$ interacting with the targets require a lot computational power otherwise, it can take many days to finish generating results. It is even more time consumming to simulate a real time spill of $5 \times 10^{12}$ protons/spill. For this reason, the gun generator is only used for relative particles studies.

- $\eta$ generator: This generator throws $\eta$ particles using PYTHIA generated distributions. The eta decays can decay into photon and a dark photon and then into a dimuon.

\subsubsection{MC simulation formats}

The Random RF trigger (NIM3) (discussed in Section 2.8) has a very important functionality in the creation of MC simulations for analysis, particularly the "Messy" MC production. The produced DY, $J / \psi$ or $\psi^{\prime}$ MC sets do not factor in background or noise coming from other muons. In order to simulate background, NIM3 data is embedded in clean Monte Carlo sets. This allows the experiment to study several background effects such as reconstruction efficiencies and the resolution of quantities. A Gaussian smearing is applied to the MC hits and $\sim 6 \%$ of them are discarded to resemble chamber efficiencies, a process referred to as "Realization". Realization is turned on before embedding the events for Messy MC and the new "embedded-hit" file is re-tracked. For Clean MC, no NIM3 events are embedded but Realization is applied. The distributions of several kinematic variables from MC productions are shown Figures 3.9 and 3.10. A comprehensive list of analysis cuts were developed via the examination of these plots. They aim to identify either high mass Drell-Yan events or low mass $J / \psi$ events coming from the target region. 


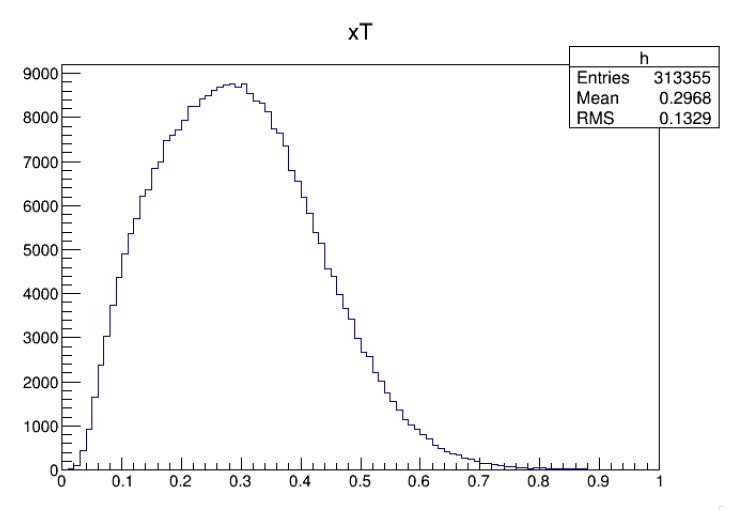

(a) DY MC for xT $\left(x_{\text {target }}\right)$

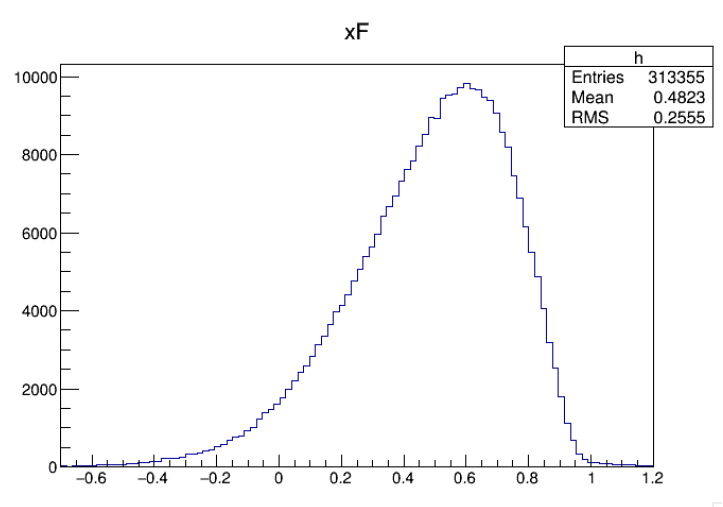

(a) DY MC for $x F(x-F e y n m a n)$

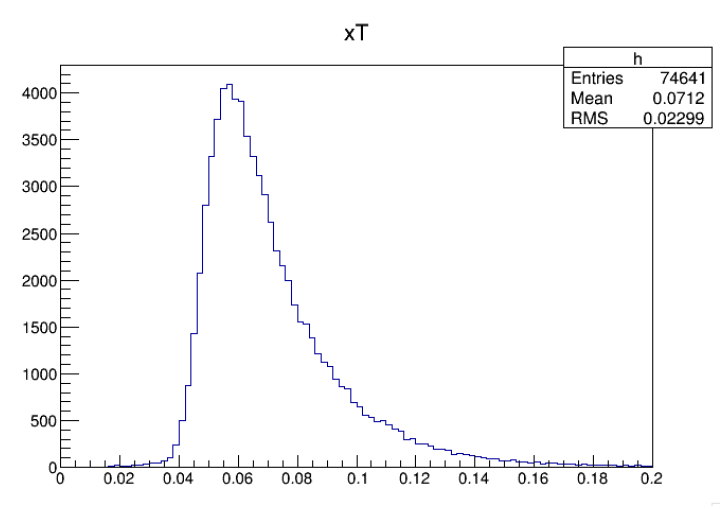

(b) $J / \psi \mathrm{MC}$ for $\mathrm{xT}\left(x_{\text {target }}\right)$

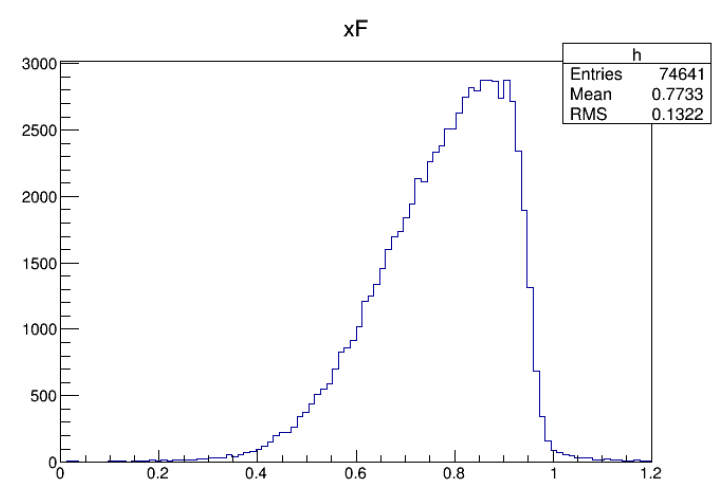

(b) $J / \psi$ MC for $\mathrm{xF}$ (x-Feynman)

Figure 3.9: Kinematic distributions of the MC events for two physics processes. 


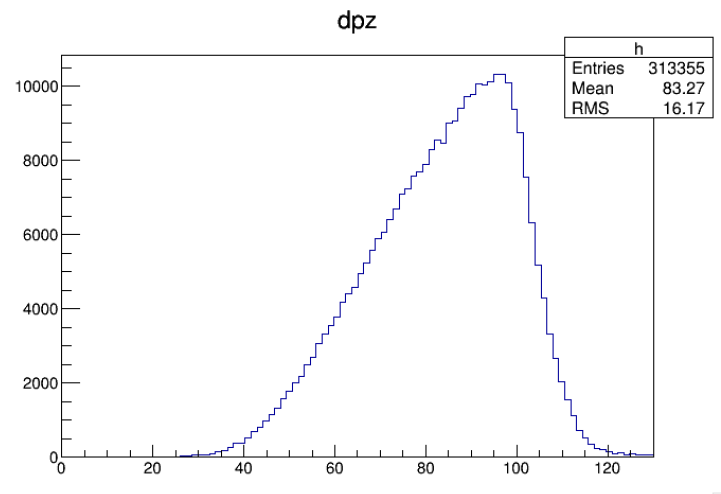

(a) DY MC for dpz

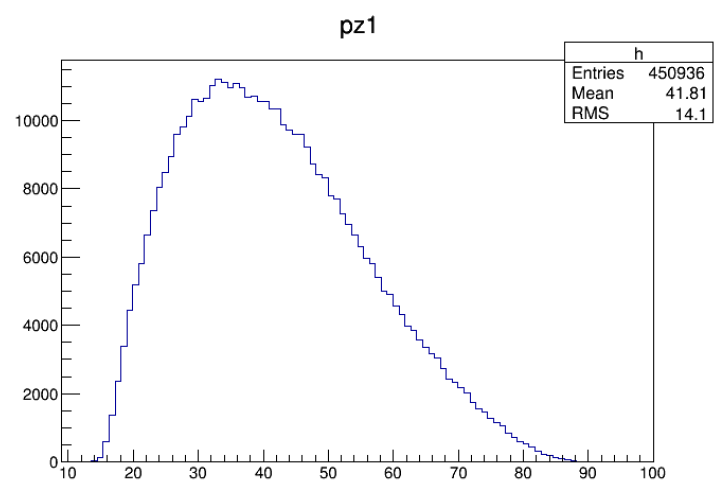

(a) DY MC for pz1

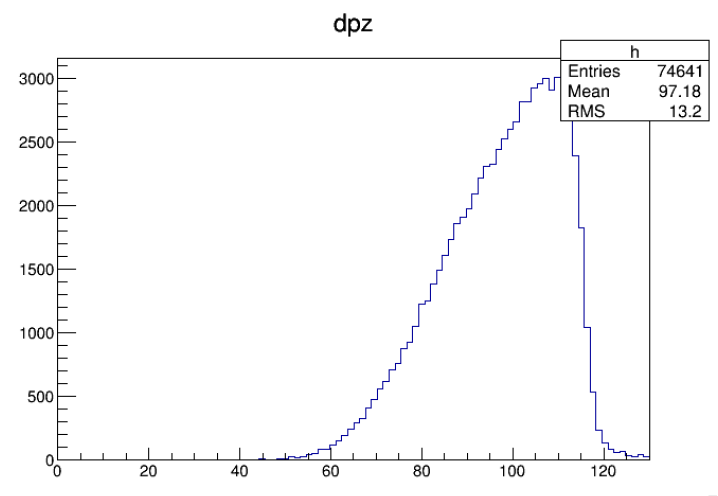

(b) $J / \psi \mathrm{MC}$ for $\mathrm{dpz}$

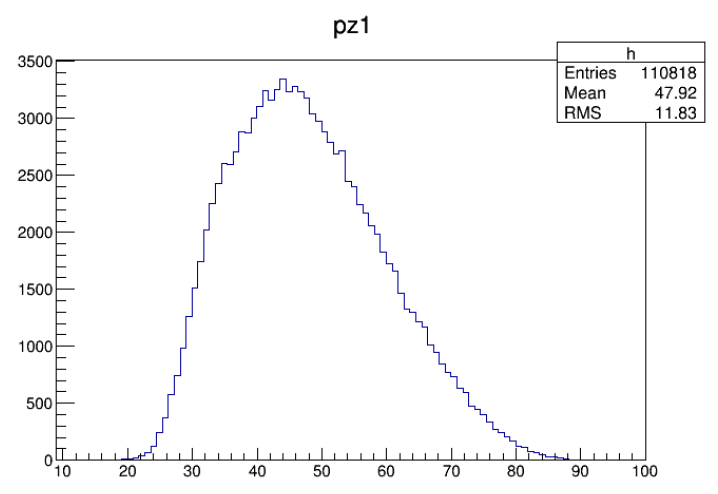

(b) $J / \psi \mathrm{MC}$ for pz1

Figure 3.10: Kinematic distributions of the MC events for two physics processes. 


\subsection{Event selection}

The track-reconstructed events have to pass through data-quality cuts constructed largely from MC studies. These cuts aim to filter out residual background events after tracking while maximizing the amount clean events available for analysis. Quality cuts are applied on several levels and are classified into spill level, event level, track level and dimuon level cuts. This section will list each set of data cuts per level used in this analysis.

\subsubsection{Spill level cuts}

\begin{tabular}{|c|c|c|c|c|}
\hline Quantity & & & Good range & 61 \\
& Roadset(RS) & $57 \& 59$ & $62,67 \& 70$ \\
\hline \hline TargetPos & & {$[1,7]$} & {$[1,7]$} & {$[1,7]$} \\
\hline TargetPos.2 & & $=$ TargetPos & $=$ TargetPos & $=$ TargetPos \\
\hline AcceptedFPGA1 & & N/A & N/A & N/A \\
\hline AfterInhFPGA1 & & N/A & N/A & N/A \\
\hline TSGo & & {$[1 \mathrm{e} 3,8 \mathrm{e} 3]$} & {$[1 \mathrm{e} 3,12 \mathrm{e} 3]$} & {$[100,6000]$} \\
\hline AcceptedFPGA1 & {$[1 \mathrm{e} 3,8 \mathrm{e} 3]$} & {$[1 \mathrm{e} 3,12 \mathrm{e} 3]$} & {$[100,6000]$} \\
\hline AfterInhFPGA1 & {$[1 \mathrm{e} 3,30 \mathrm{e} 3]$} & {$[1 \mathrm{e} 3,1000 \mathrm{e} 3]$} & {$[100,10000]$} \\
\hline AcceptedFPA1/AfterInhFPGA1 & & {$[0.2,0.9]$} & {$[0.0,0.9]$} & {$[0.2,1.05]$} \\
\hline FMAG & & {$[1950,2050]$} & {$[200,500]$} & {$[1950,2050]$} \\
\hline KMAG & {$[1550,1650]$} & {$[1550,1650]$} & {$[1550,1650]$} \\
\hline G2SEM & & {$[2 \mathrm{e} 12,1 \mathrm{e} 13]$} & {$[2 \mathrm{e} 12,1 \mathrm{e} 13]$} & {$[2 \mathrm{e} 12,1 \mathrm{e} 13]$} \\
\hline QIEsum & {$[4 \mathrm{e} 10,1 \mathrm{e} 12]$} & {$[4 \mathrm{e} 10,1 \mathrm{e} 12]$} & {$[4 \mathrm{e} 10,1 \mathrm{e} 12]$} \\
\hline Inhibit & {$[4 \mathrm{e} 9,1 \mathrm{e} 11]$} & {$[4 \mathrm{e} 9,1 \mathrm{e} 11]$} & {$[4 \mathrm{e} 9,2 \mathrm{e} 11]$} \\
\hline Busy & & {$[4 \mathrm{e} 9,1 \mathrm{e} 11]$} & {$[4 \mathrm{e} 9,1 \mathrm{e} 11]$} & {$[4 \mathrm{e} 9,1 \mathrm{e} 11]$} \\
\hline Duty Factor & & {$[15,60]$} & {$[15,60]$} & {$[10,60]$} \\
\hline N of tracks/spill & & $>0$ & $>0$ & $>0$ \\
\hline
\end{tabular}

Table 3.4: "Good spill" specifications from [4]

These cuts are used to discard data from proton spills that have irregular scale readings or unreliable recorded process variables. For spills satisfying minimum requirements a useful data quality bit, "Spill.dataQuality $=0$ ", is used to identify and gather qualifying spills. A list of all the cuts representing this data quality bit are shown in Table 3.4. 


\subsubsection{Event level cuts}

These cuts have been optimized primarily to realize sufficient target vs. beam-dump separation. In addition, by studying low and high DC1 occupancies, they are also tuned to remove randoms. As noted before, the beam offset changed between RS 57, 59 and 62, 67, 70 and 78. The magnetic field was also flipped at the beginning of RS 67. This analysis has these RS-dependent features applied prior to any event evaluation. All DY and $J / \psi$ analyses share these cuts unless explicitly stated otherwise.

- Occupancy: The occupancy of the event at DC1, DC2, DC3 and the occupancy sum of all should be less than a given value to study events that are reconstructable.

$$
\begin{aligned}
& -\mathrm{D} 1<400 \\
& -\mathrm{D} 2<400 \\
& -\mathrm{D} 3<400 \\
& -\mathrm{D} 1+\mathrm{D} 2+\mathrm{D} 3<1000
\end{aligned}
$$

- Trigger requirement: This cut is applied in order to guarantee that each muon in the vertexed pair stemmed purely from either the top or bottom halves of the spectrometer. Ultimately, the product of the vertical positions of $\mu^{+}$and $\mu^{-}$at DC3 should be less than zero in order to fulfill the $\mathrm{B} / \mathrm{T}$ or $\mathrm{T} / \mathrm{B}$ requirement of the FPGA1 dimuon trigger.

$$
\begin{aligned}
& -y 1_{-} \mu^{+} * y 3_{-} \mu^{+}>0 \\
& -y 1_{-} \mu^{-} * y 3_{-} \mu^{-}>0 \\
& -y 3_{-} \mu^{+} * y 3_{-} \mu^{-}<0
\end{aligned}
$$

\subsubsection{Track level cuts}

DY and $J / \psi$ analyses share these cuts unless explicitly stated otherwise. 
- $z 0$ : These relate to the distance of closest approach to the beam-line. These cuts are used to minimize the contributions coming from the upstream instrumentation package and the downstream tail of events coming from the iron beam dump.

$$
\begin{aligned}
& --320 \mathrm{~cm}<z 0_{-} \mu^{+}<-5 \mathrm{~cm} \\
& --320 \mathrm{~cm}<z 0_{-} \mu^{-}<-5 \mathrm{~cm}
\end{aligned}
$$

- $x T, y T, x D, y D$ : These are the projected $x$ and $y$ positions at $z=-129 \mathrm{~cm}(x T$ and $y T)$ and $z=42 \mathrm{~cm}(x D$ and $y D) . z=+42 \mathrm{~cm}$ is chosen as it is one interaction length $(17 \mathrm{~cm})$ from the hole in the front face of the beam dump $(25 \mathrm{~cm})$.

$$
\begin{aligned}
& -x T * x T+(y T-\text { beamoffset }) *(y T-\text { beamoffset })<320 \mathrm{~cm}^{2} \\
& -x D * x D+(y D \text { - beamoffset }) *(y D-\text { beamoffset })<1100 \mathrm{~cm}^{2} \\
& -x D * x D+(y D-\text { beamoffset }) *(y D-\text { beamoffset })>16 \mathrm{~cm}^{2}
\end{aligned}
$$

- KMAG $p_{T}$ kick: $p x 1$ and $p x 3$ are the $x$ component of the three momenta at DC1 and DC3, respectively. This quantity is guided by the magnetic field between DC1 and DC3. So that the correct $p_{T}$ is applied at KMAG cuts are applied for both muons in the vertexed pair.

$$
\begin{aligned}
& \text { - } \operatorname{abs}(\operatorname{abs}(p x 1-p x 3)-0.416)<0.008 \mathrm{GeV} / \mathrm{c} \\
& \text { - } \operatorname{abs}(p y 1-p y 3)<0.008 \mathrm{GeV} / \mathrm{c} \\
& \text { - } a b s(p z 1-p z 3)<0.08 \mathrm{GeV} / \mathrm{c}
\end{aligned}
$$

- chisq_upstream, chisq_target, chisq_dump: These are used to separate target tracks from dump tracks. Three $\chi^{2}$ 's are given out when the track is forced to go through $x=y=0$ and $z=-490 \mathrm{~cm},-129 \mathrm{~cm}$ and $42 \mathrm{~cm}$ [39]. For target DY or $J / \psi$ dimuons, $\chi^{2}$ for the target should be distinguishably less than the $\chi^{2}$ for the dump and upstream. In addition, an upper limit on the track $\chi^{2}$ is applied in order to limit the number of badly reconstructed tracks still, making it through the loose upper limits of the internal tracker cuts. 
- chisq_target $<1.5 *$ chisq_dump

- chisq_target $<15$

- abs(chisq_target_ $\mu^{+}+$chisq_target_ $\mu^{-}-$chisq_dimuon $)<2$

- numHits: Normally, a track has 16,17 or 18 hits, given that a few can be dropped due to hardware efficiency, track reconstruction inefficiency and other rate dependence effects. It is unlikely that a "good" track has 13 hits. In addition, according to MC studies, an upper limit is placed on the total number of hits on each track for the dimuons and the number of hits of both the tracks at DC1.1.

$$
\begin{aligned}
& \text { - } \text { numHits_ }_{-}^{+}>13 \\
& \text { - } \text { numHits_ } \mu^{-}>13 \\
& \text { - numHits } \mu_{-} \mu^{+}+\text {numHits } \mu_{-}^{-}>29 \\
& \text { - numHits_ } \mu^{+} \__{2} a t_{-} D C 1+\text { numHits } \mu_{-} \mu_{-} a t \_D C 1>8
\end{aligned}
$$

- chisq/(numHits - 5): The track is specified by 5 variables: $x_{B}, x_{T}, p_{T}, \phi, \theta$. These also represent the degrees of freedom of the track. An upper limit is placed on the $\chi^{2} / \mathrm{NDF}$ in order to guarantee good quality reconstructed tracks.

- chisq/(numHits -5$)<12$

- $p z 1$ : This variable is the $z$ component of the three-momentum of a muon at DC1.

$$
\begin{aligned}
& -9<p z 1_{-} \mu^{+}<75 \mathrm{GeV} / \mathrm{c} \\
& -9<p z 1_{-} \mu^{-}<75 \mathrm{GeV} / \mathrm{c}
\end{aligned}
$$

- Rejecting cross-over muons: These cuts guarantee that the vertical position of the muons at DC3 is greater than at St1. This is applied in order to prevent the use of muons that could have from crossed from the top half to the bottom half of the spectrometer.

$$
\begin{aligned}
& \text { - } y 1_{\mu^{+}} / y 3_{\mu^{+}}<1 \\
& \text { - } y 1_{\mu^{-}} / y 3_{\mu^{-}}<1
\end{aligned}
$$




\subsubsection{Dimuon level cuts}

- $d x, d y, d z$ : These define the dimuon vertex position from vertex finding. The cuts attempt to reduce the contributions coming from the upstream instrumentation package and the downstream tail of events, specifically from the iron beam dump.

$$
\begin{aligned}
& --280 \mathrm{~cm}<d z<-5 \mathrm{~cm} \\
& -a b s(d y-\text { beamoffset })<0.22 \mathrm{~cm} \text { (beam offset }=0.4 \mathrm{~cm} \text { or } 1.6 \mathrm{~cm} \text { depending on the } \\
& \quad \mathrm{RS}) \\
& -a b s(d x)<0.25 \mathrm{~cm} \\
& -d x * d x+(d y-\text { beamoffset }) *(d y-\text { beamoffset })<0.06 \mathrm{~cm}^{2}
\end{aligned}
$$

- $d p x, d p y, d p z:$ These define the dimuon three-momenta at vertex position after re-tracking.

$$
\begin{aligned}
& \text { - } a b s(d p x)<1.8 \mathrm{GeV} / \mathrm{c} \\
& \text { - } a b s(d p y)<2 \\
& \text { - DY: } 38 \mathrm{GeV} / \mathrm{c}<a b s(d p z)<120 \mathrm{GeV} / \mathrm{c} \\
& \text { - } J / \psi: 60 \mathrm{GeV} / \mathrm{c}<a b s(d p z)<120 \mathrm{GeV} / \mathrm{c} \\
& \text { - } \left.d p x * d p x+d p y * d p y<5(\mathrm{GeV} / \mathrm{c})^{2} \text { (loose cut on } p_{T}^{2}\right)
\end{aligned}
$$

- mass: This is the calculated dimuon mass.

$$
\begin{aligned}
& \text { - DY: } 4.2 \mathrm{GeV} / \mathrm{c}^{2}<\text { mass }<8.8 \mathrm{GeV} / c^{2}
\end{aligned}
$$

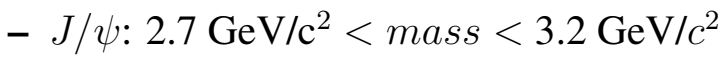

- $x_{F}$ : This is the calculated Feynman- $x$.

$$
\begin{aligned}
& \text { - DY: }-0.1<x_{F}<0.95 \\
& \text { - } J / \psi: 0.4<x_{F}<0.95
\end{aligned}
$$

- $x_{T}$ : This is the calculated Bjorken- $x$. 
- DY: $0.1<x_{T}<0.58$

- $J / \psi: 0.04<x_{T}<0.13$

- $\cos (\theta)$ : This is the polar angle in the Collins-Soper frame.

$-\operatorname{abs}(\cos (\theta))<0.5$

- trackSeparation: This is the distance between the points of closest approach between muons of the vertexed pair: $\mu^{+}$and $\mu^{-}\left(z_{-} \mu^{+}-z_{-} \mu^{-}\right)$.

- abs(trackSeparation $)<270 \mathrm{~cm}$

- chisq_dimuon: This is the $\chi^{2}$ imposing that both muons to go through the dimuon vertex. An upper limit is placed to guarantee that "good" reconstructed dimuons are available for analysis.

- chisq_dimuon $<18$

- Trigger Intensity: Trigger Intensity refers to the number of protons in the triggered RF bucket. The expression for calculating the trigger intensity (\# of protons in the triggered $\mathrm{RF}$ bucket - RF00) range is:

$$
\begin{gathered}
\text { TriggerIntensity }=(\text { RF00 }- \text { pedestal }) * \frac{\text { G2SEM }}{(\text { QIEsum }- \text { pedestal } * \text { buckets } * \text { turns })} \\
\text { PotPerQie }=\frac{\text { G2SEM }}{(\text { QIEsum }- \text { pedestal } * \text { buckets } * \text { turns })}
\end{gathered}
$$

The QIE module reads out the pedestal value for all the buckets, including empty buckets. In a spill, the number of buckets are 588 and the number of turns are 369,000 . For a spill range $<450,000$, a QIE pedestal value of 36.2 was taken. A pedestal value of 32.6 was taken from subsequent spills.

- $($ RF00 - pedestal $) *$ PotPerQie $>0$ 
- $($ RF00 - pedestal $) *$ PotPerQie $<64000$

- Reduction of random background: In the spectrometer, muons fire most frequently near the beam-line, although most of these come from the beam dump. By examining MC, data, low and high DC1 occupancy distributions, a limit was placed on the vertical component of the momentum and the $x$ position of muons at DC1.

$$
\begin{aligned}
& \text { - } \operatorname{abs}\left(x 1_{-} \mu^{+}+x 1_{-} \mu^{-}\right)<42 \mathrm{~cm} \\
& \text { - } \operatorname{abs}\left(p y 1_{-} \mu^{+}\right)>0.02 \mathrm{GeV} / \mathrm{c} \\
& \text { - } \operatorname{abs}\left(p y 1_{-} \mu^{-}\right)>0.02 \mathrm{GeV} / \mathrm{c}
\end{aligned}
$$

\subsection{Invariant mass spectrum}

An invariant mass spectrum of carbon is shown in Figure 3.11. The fit incorporates $J / \psi, \psi^{\prime}$ and DY MC along with a background spectrum which is simulated only using the FPGA4 events (labeled "Mixed" on plot). In this analysis, mass spectrum fits were used to estimate a physics signal contamination factor, $F$, for the $J / \psi$ signal described in detail in Section 3.9.5. 


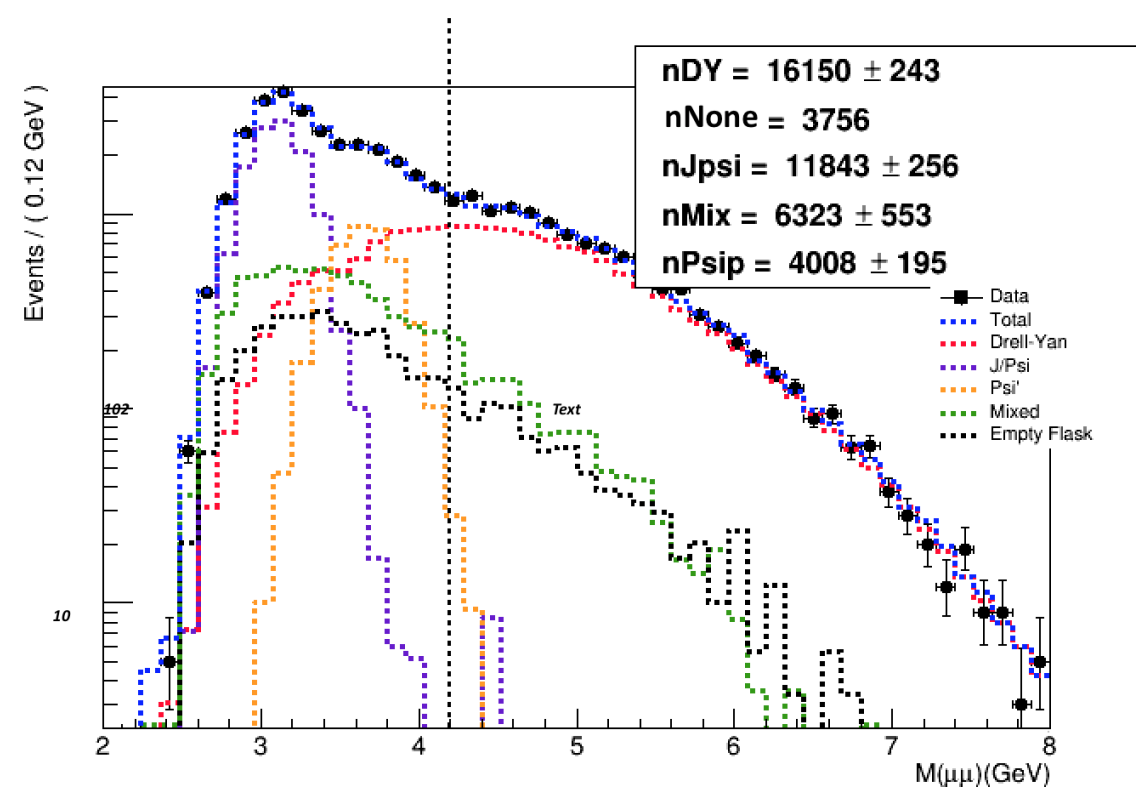

Figure 3.11: An invariant mass spectrum plot for carbon. Values on the upper right hand corner (nDY, nFlask...) are normalization values for each component provided by the fit. The dotted line indicates where the lower mass cut takes place for DY events.

\subsection{Normalized yields for different kinematic variables}

Normalized yields as a function of different kinematic variables were made for both DY and $J / \psi$ analyses. The differences between roadsets are within resolution of each respective variable allowing for RS 57, 59, 62, 67 and 70 to be combined for analysis from this point forward (all results contain data from all RS). Figures 3.12 - 3.23 show the normalized yields of some the primary variables for these analyses for liquid and solid targets, as well as empty flask and "no target" data. In general, the shapes of all the distributions don't depend on the target type, with some differences in the empty flask and "no target" distributions largely due to low statistics. See Appendix A.1 for all raw dimuon yield tables for $p_{T}$ and $x_{F}$ bins.

\subsection{1 $J / \psi$ plots of normalized yields}




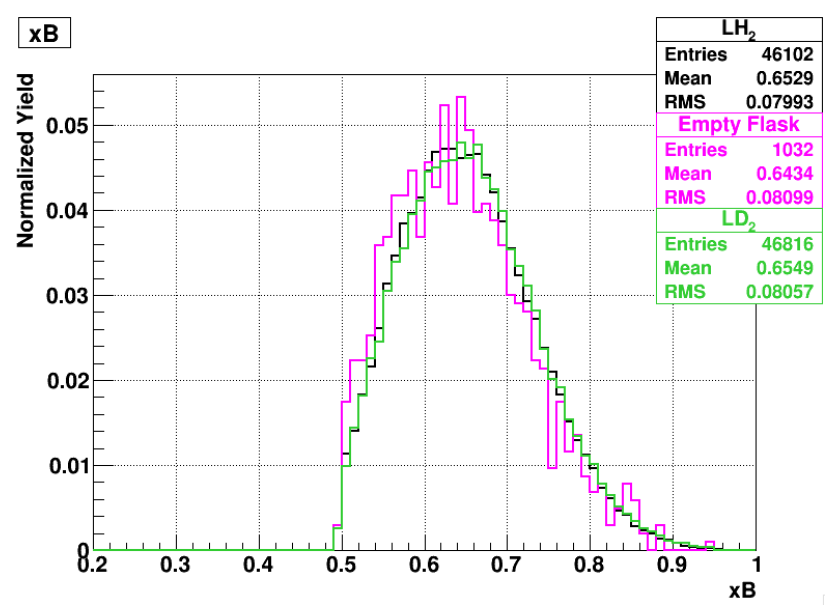

(a) Liquid targets

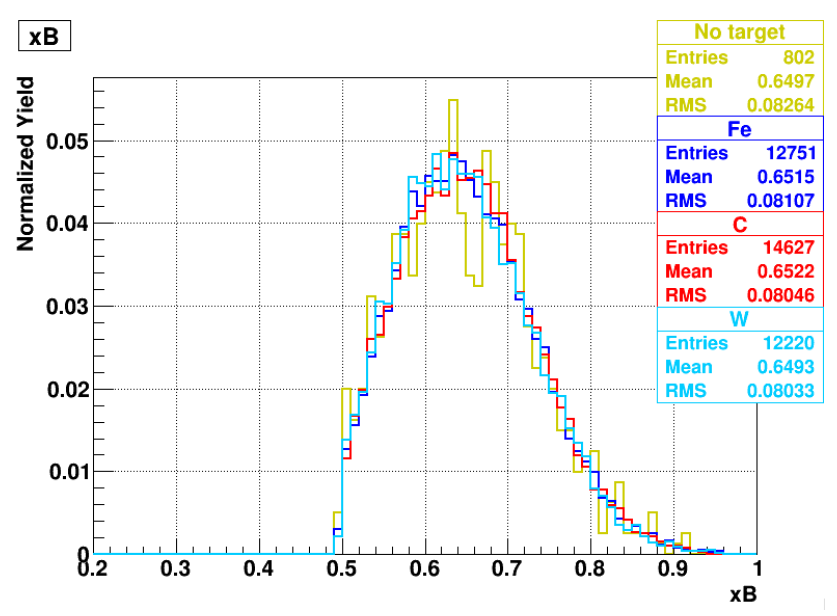

(b) Solid targets

Figure 3.12: $\mathrm{J} / \psi$ normalized yields for $x_{B}$ : (a) $\mathrm{LH}_{2}$, Empty flask and $\mathrm{LD}_{2}$ and b) No target, Fe, $\mathrm{C}$ and $\mathrm{W}$ targets. 


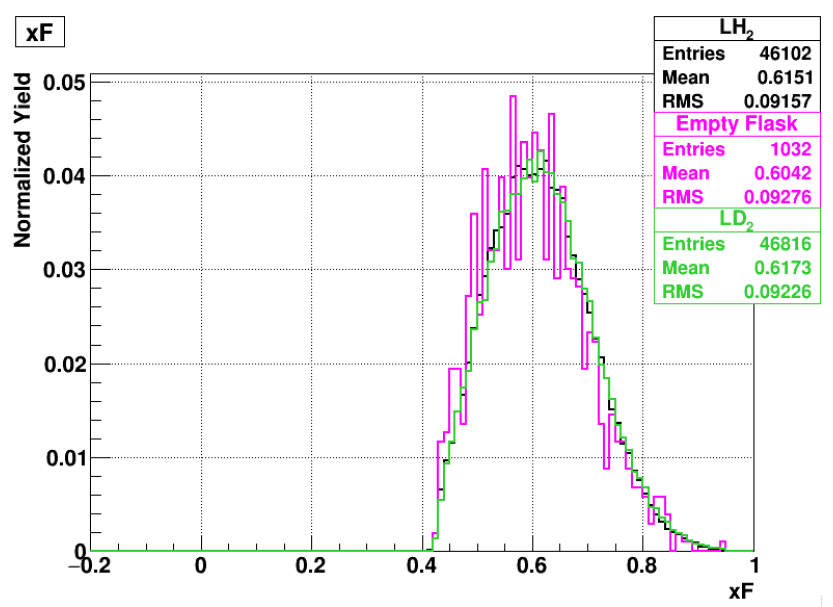

(a) Liquid targets

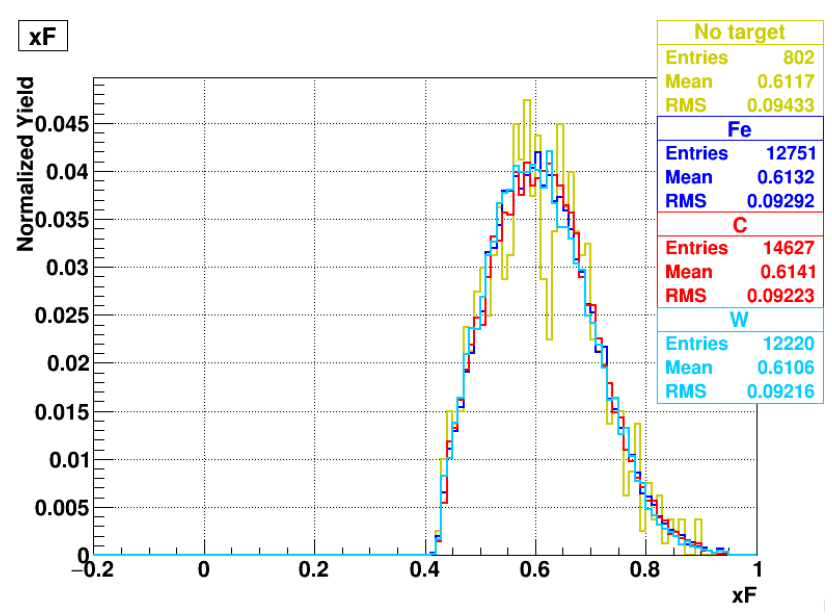

(b) Solid targets

Figure 3.13: $J / \psi$ normalized yields for $x_{F}$ : (a) $\mathrm{LH}_{2}$, Empty flask and $\mathrm{LD}_{2}$ and b) No target, Fe, C and $\mathrm{W}$ targets. 


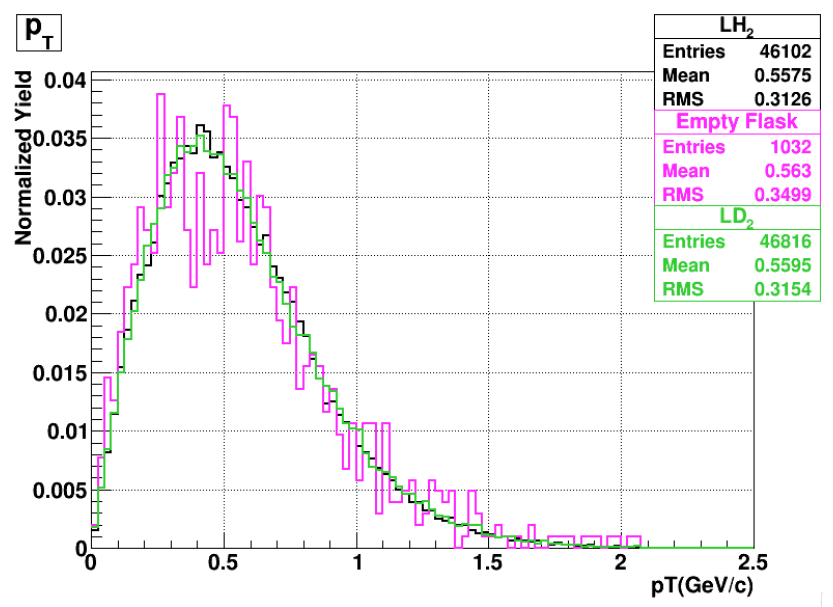

(a) Liquid targets

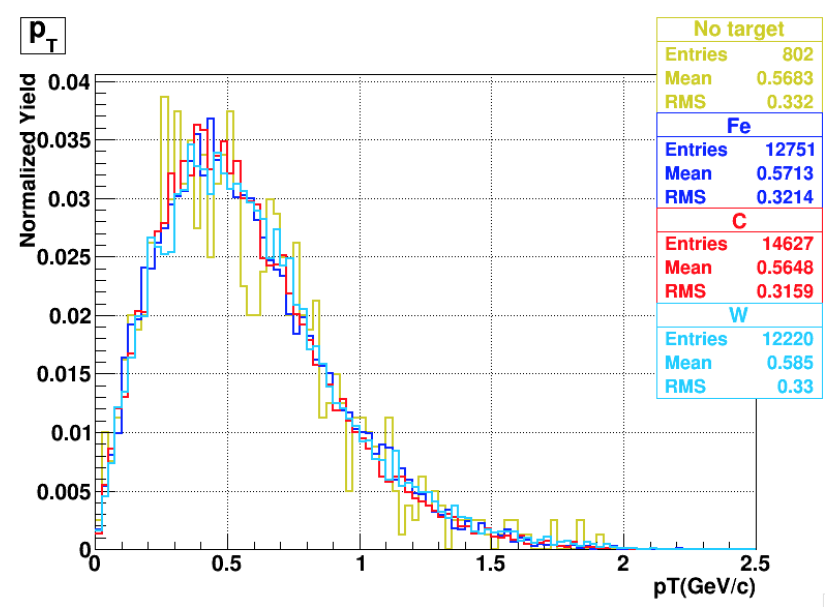

(b) Solid targets

Figure 3.14: $\mathrm{J} / \psi$ normalized yields for $p_{T}$ : (a) $\mathrm{LH}_{2}$, Empty flask and $\mathrm{LD}_{2}$ and b) No target, Fe, $\mathrm{C}$ and $\mathrm{W}$ targets. 


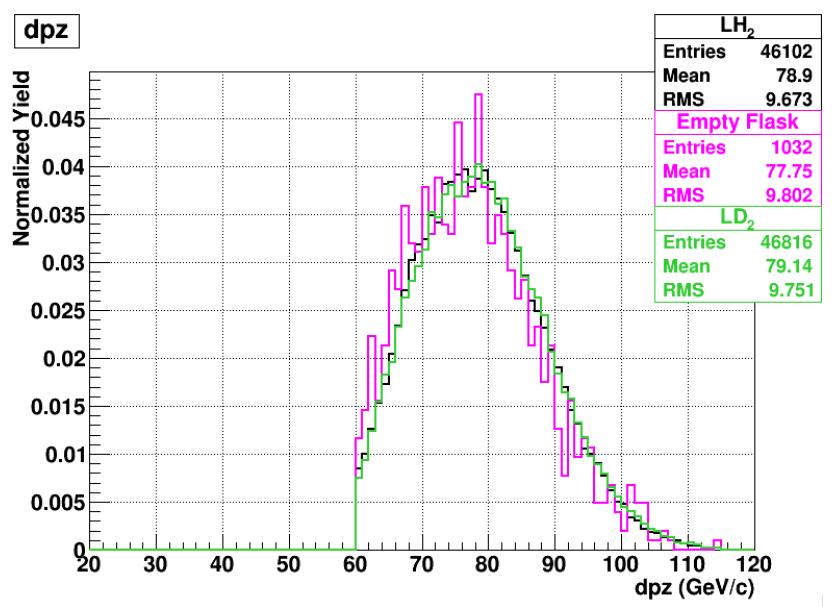

(a) Liquid targets

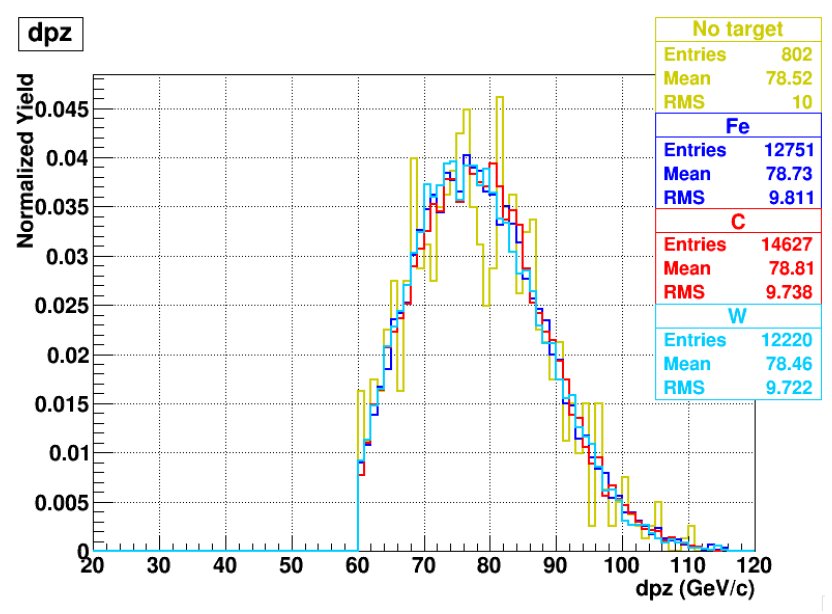

(b) Solid targets

Figure 3.15: $J / \psi$ normalized yields for $d p z$ : (a) $\mathrm{LH}_{2}$, Empty flask and $\mathrm{LD}_{2}$ and b) No target, Fe, $\mathrm{C}$ and $\mathrm{W}$ targets. 


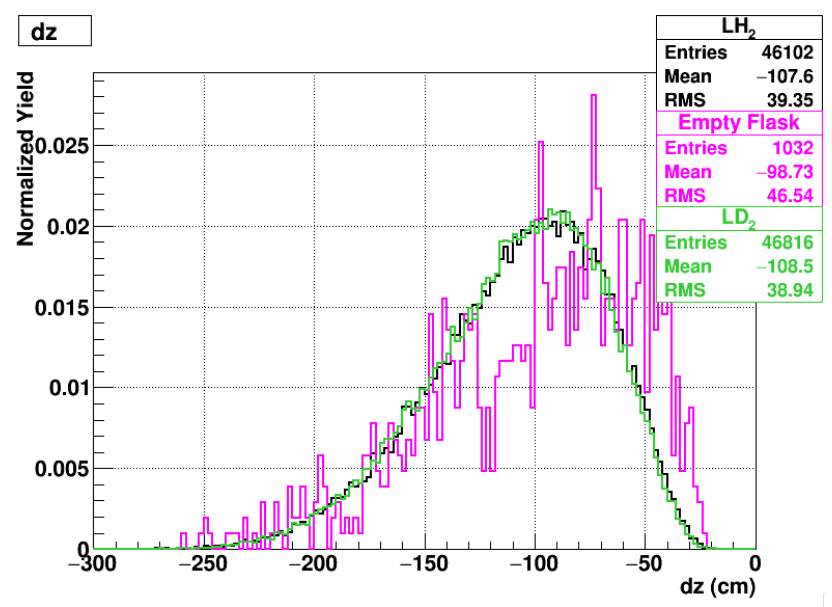

(a) Liquid targets

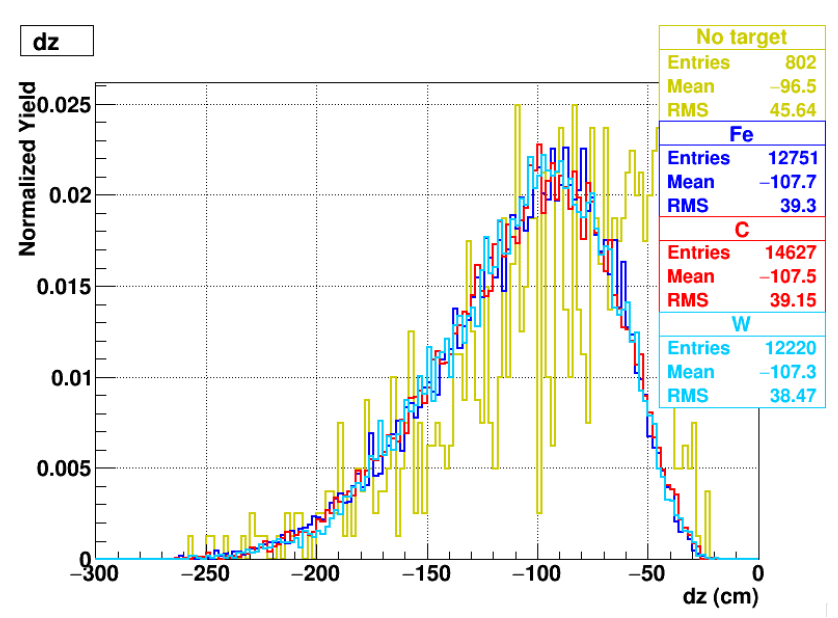

(b) Solid targets

Figure 3.16: $J / \psi$ normalized yields of $d z$ (dimuon vertex) for (a) $\mathrm{LH}_{2}$, Empty flask and $\mathrm{LD}_{2}$ and b) No target, Fe, C and W targets. 


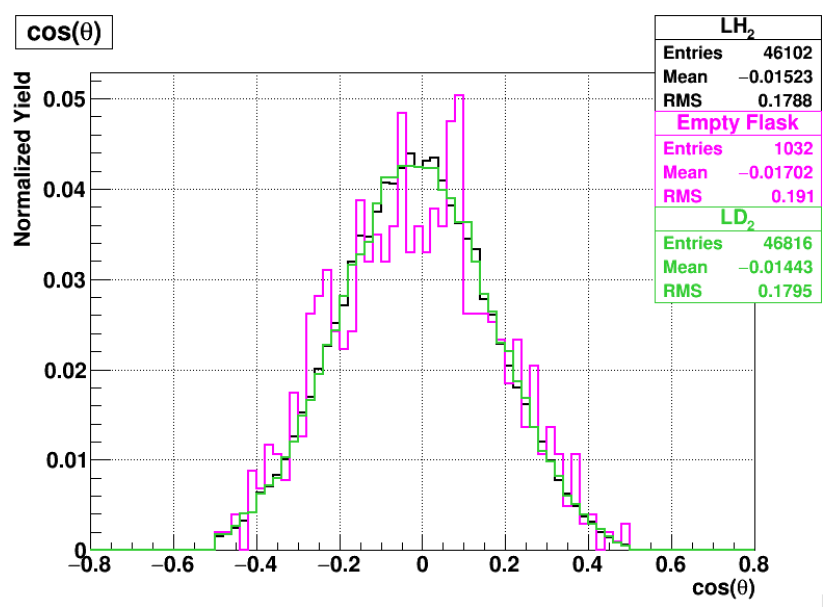

(a) Liquid targets

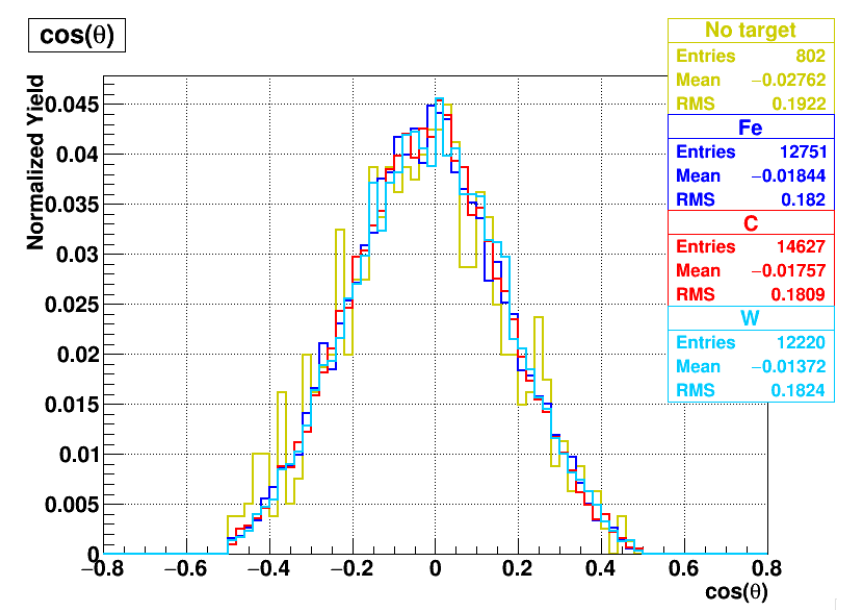

(b) Solid targets

Figure 3.17: $J / \psi$ normalized yields for $\cos \theta$ : (a) $\mathrm{LH}_{2}$, Empty flask and $\mathrm{LD}_{2}$ and b) No target, Fe, $\mathrm{C}$ and $\mathrm{W}$ targets. 


\subsubsection{DY plots of normalized yields}

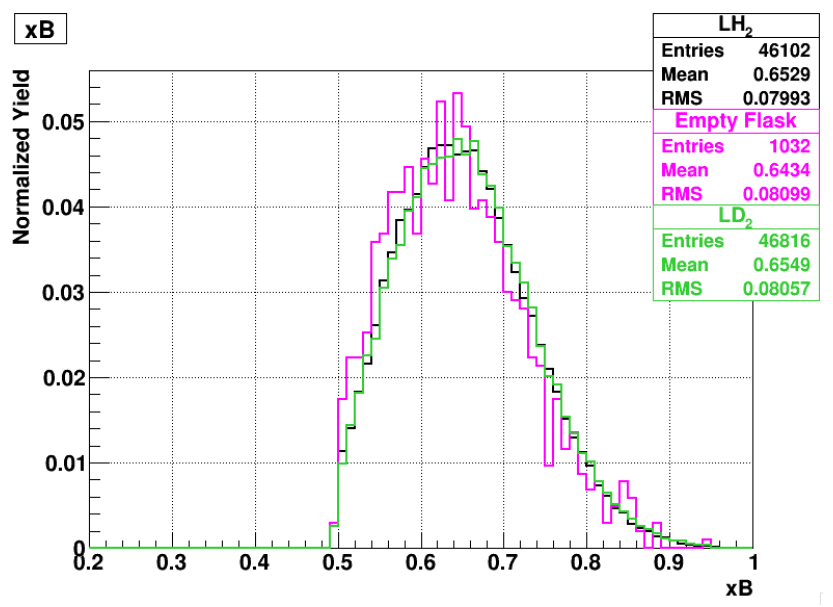

(a) Liquid targets

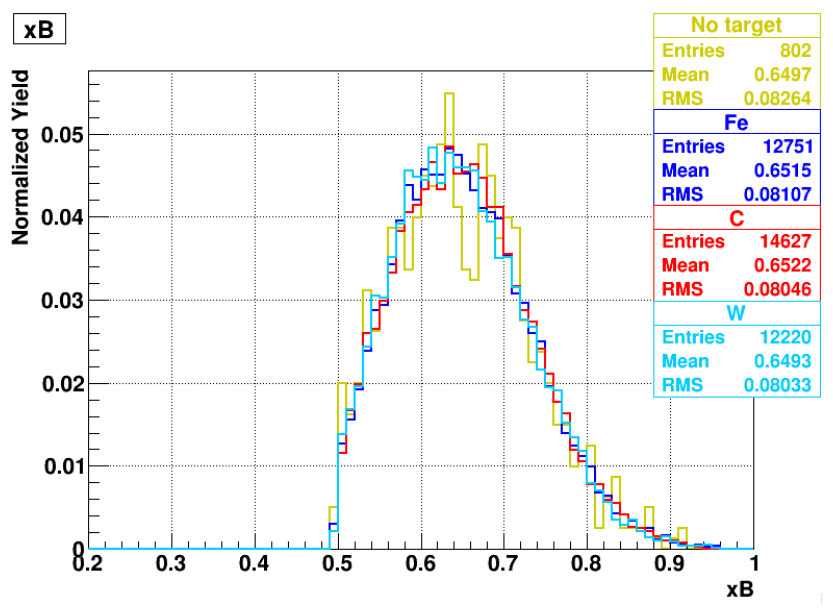

(b) Solid targets

Figure 3.18: DY normalized yields for $x_{B}$ : (a) $\mathrm{LH}_{2}$, Empty flask and $\mathrm{LD}_{2}$ and b) No target, Fe, $\mathrm{C}$ and $\mathrm{W}$ targets. 


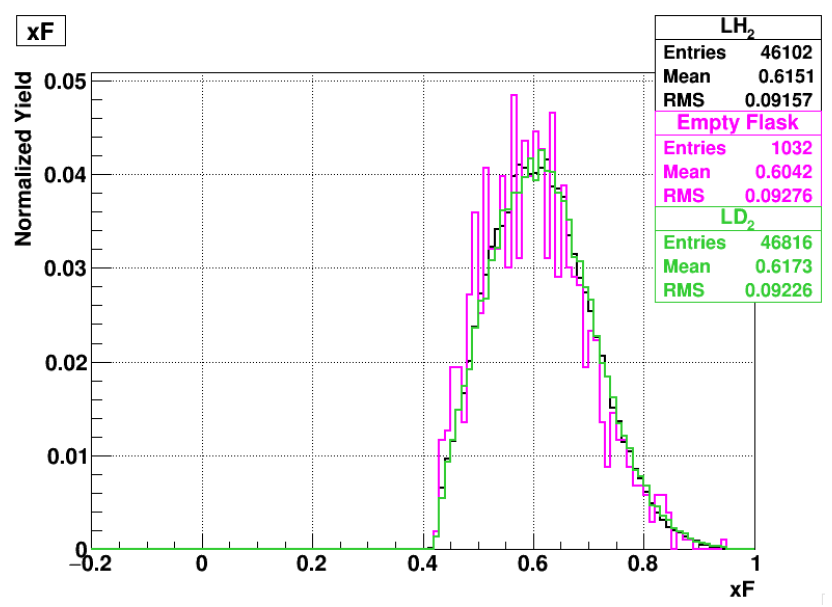

(a) Liquid targets

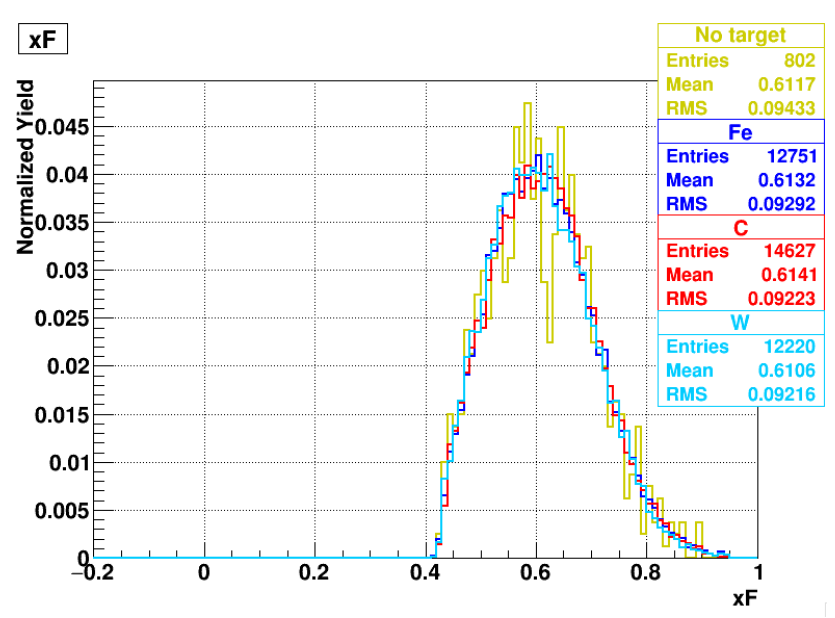

(b) Solid targets

Figure 3.19: DY normalized yields for $x_{F}$ : (a) $\mathrm{LH}_{2}$, Empty flask and $\mathrm{LD}_{2}$ and b) No target, $\mathrm{Fe}, \mathrm{C}$ and $\mathrm{W}$ targets. 


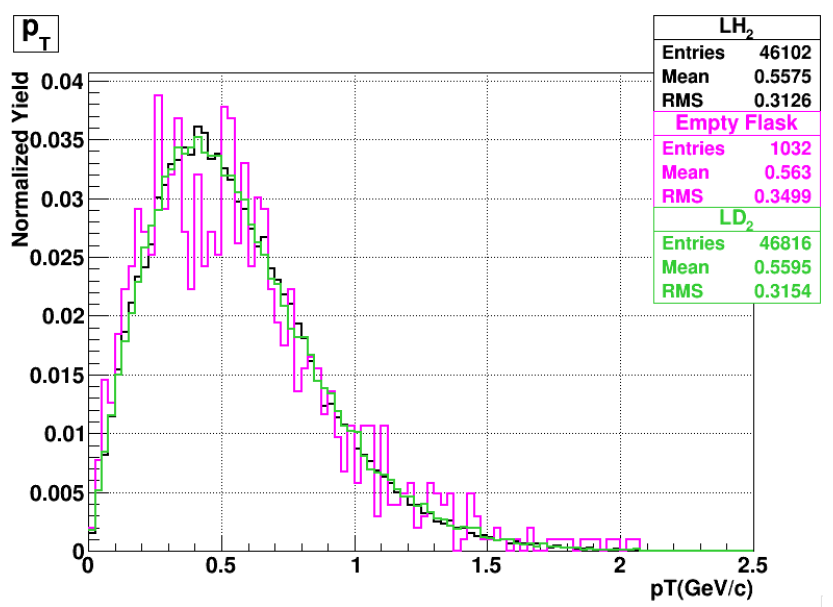

(a) Liquid targets

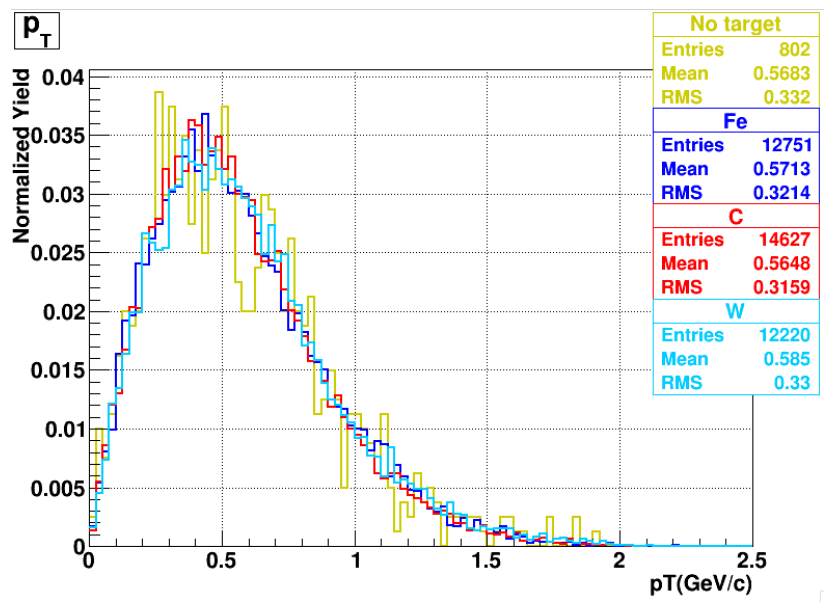

(b) Solid targets

Figure 3.20: DY normalized yields for $p_{T}$ : (a) $\mathrm{LH}_{2}$, Empty flask and $\mathrm{LD}_{2}$ and b) No target, Fe, C and $\mathrm{W}$ targets. 


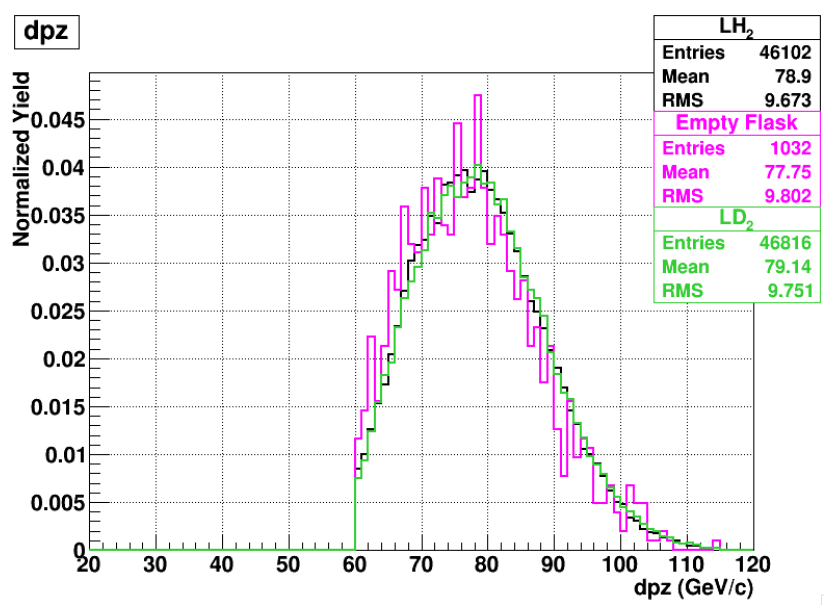

(a) Liquid targets

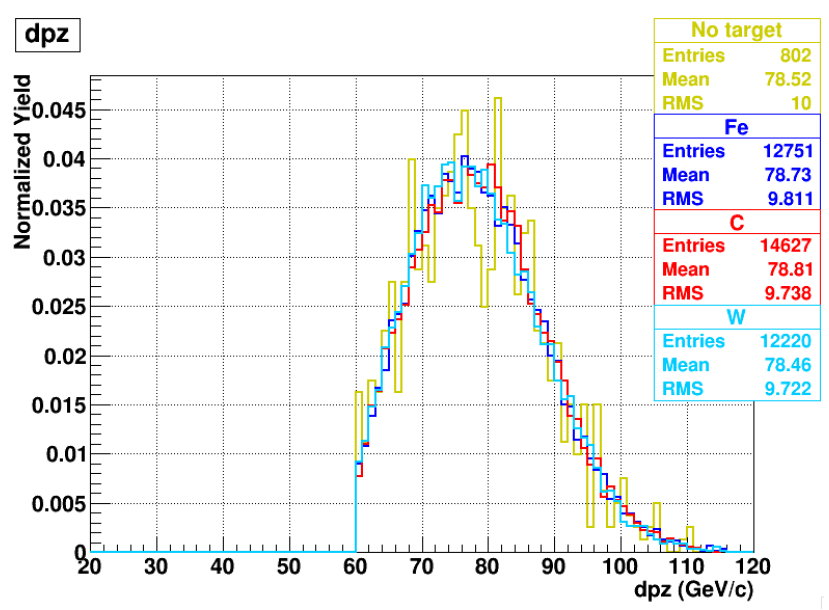

(b) Solid targets

Figure 3.21: DY normalized yields for $d p z$ : (a) $\mathrm{LH}_{2}$, Empty flask and $\mathrm{LD}_{2}$ and b) No target, Fe, C and $\mathrm{W}$ targets. 


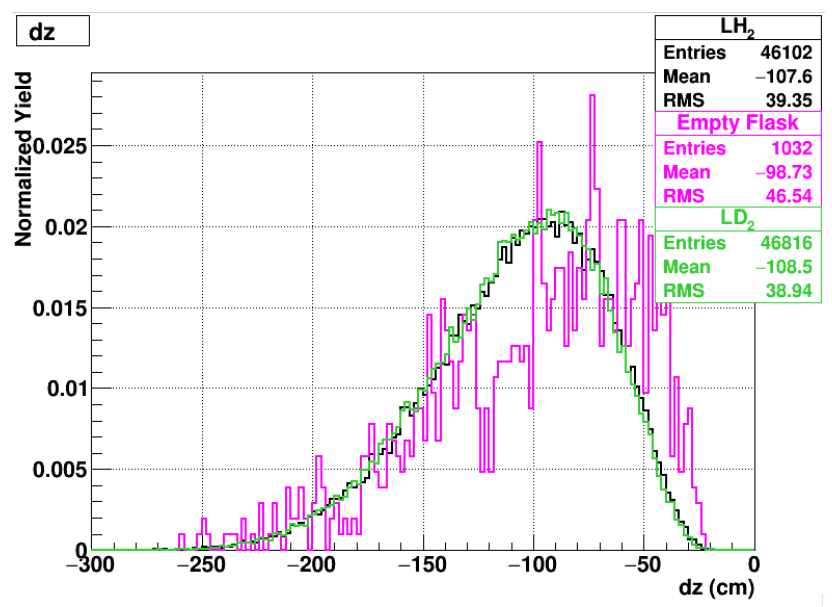

(a) Liquid targets

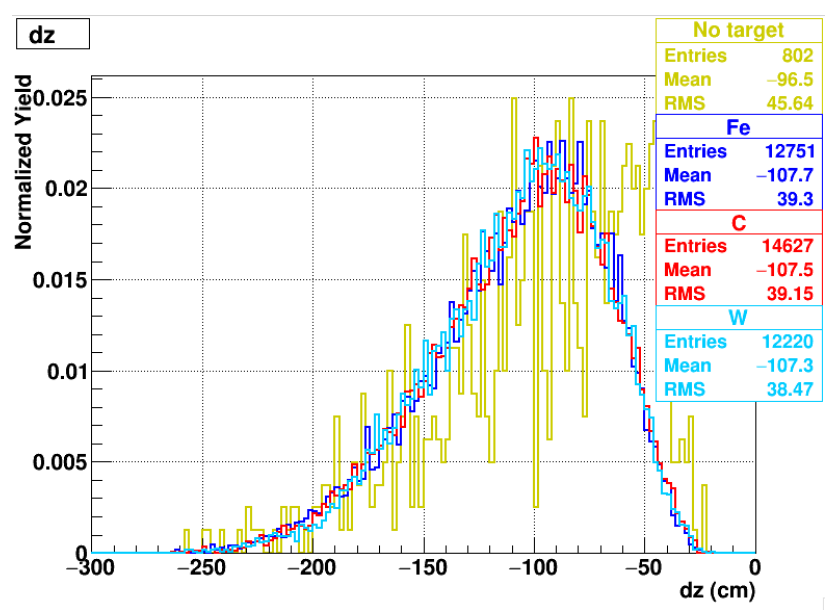

(b) Solid targets

Figure 3.22: DY normalized yields of $d z$ (dimuon vertex) for (a) $\mathrm{LH}_{2}$, Empty flask and $\mathrm{LD}_{2}$ and b) No target, Fe, C and W targets. 


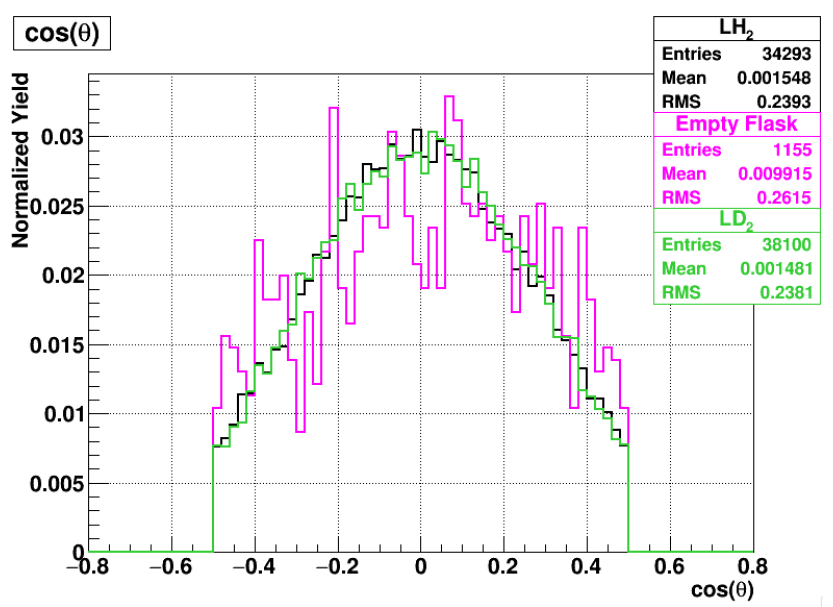

(a) Liquid targets

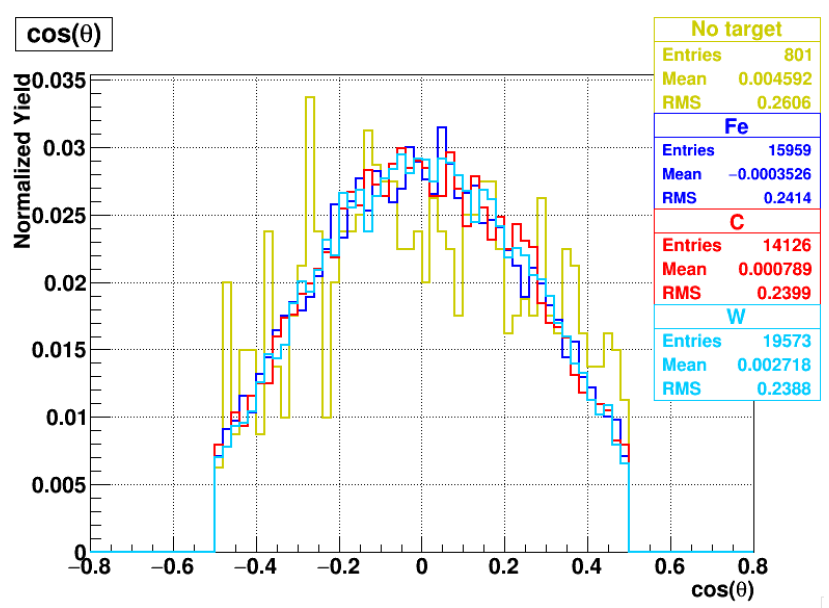

(b) Solid targets

Figure 3.23: DY normalized yields for $\cos \theta$ : (a) $\mathrm{LH}_{2}$, Empty flask and $\mathrm{LD}_{2}$ and b) No target, Fe, $\mathrm{C}$ and $\mathrm{W}$ targets. 


\subsection{Liquid target corrections}

These raw yields now need to undergo a series of target-related corrections before they are ready to be used in any per proton cross-section ratio analysis. This section will cover these corrections in detail.

\section{Target contamination corrections}

Information relevant to this correction are as follows:

- Liquid targets are $50.8 \mathrm{~cm}$ long and $7.62 \mathrm{~cm}$ in diameter

- Each flask can hold about 2.2 liters of liquid

- Flask walls are made of $76 \mu \mathrm{m}$-thick, stainless steel with $51 \mu \mathrm{m}$-thick stainless steel end caps

- For the gas used in the liquid hydrogen (" $L \mathrm{H}_{2}$ ") and liquid deuterium (" $L D_{2}$ ") target see Section 2.3.2

- HD (hydrogen deuteride) molecules are roughly $9.4 \%$ higher in volume than $\mathrm{D}_{2}$ molecules (or $\mathrm{H}_{2}$ molecules) and the density of $\mathrm{H}$ used in HD has to be adjusted accordingly

- The density of deuterium and hydrogen were taken to be $0.164 \mathrm{gm} / \mathrm{cc}$ and 0.0708 gm/cc, respectively [40]. The contamination of hydrogen is minor, relative to deuterium, and is thus disregarded (Fraction $\mathrm{H}_{2}$ is set to 0$)$ :

- For the complete details of this contamination correction procedure, see [41]

The volume of the mixed deuterium can be written as the following expression:

$$
\begin{gathered}
V_{c}=\text { Fraction }_{2} * 1.0+\text { FractionHD } * 1.094+\text { Fraction }_{2} * 1.221 \\
=0.918+0.082 * 1.094+0 * 1.221=1.007708 \\
\left\langle V_{c}\right\rangle=\frac{\text { Raw }_{\text {cont }} * 1.007708+\text { Raw }_{\text {pure }} * 1}{\text { Raw }_{\text {Total }}}
\end{gathered}
$$


Thus, the weighted (by RAW:G2SEM) average of several quantities is:

$$
\langle D\rangle=\frac{\operatorname{Raw}_{\text {cont }} *(1-c)+\operatorname{Raw}_{\text {pure }} * 1}{\operatorname{Raw}_{\text {Total }}}
$$

where $\langle D\rangle$ is the molecular fraction of $\mathrm{D}$ of pure deuterium in the contaminated $\mathrm{LD}_{2}$ and $c$ refers to the percentage of contamination.

$$
\langle H D\rangle=\frac{\operatorname{Raw}_{\text {cont }} * c+\text { Raw }_{\text {pure }} * 0}{\text { Raw }_{\text {Total }}}
$$

where $\langle H D\rangle$ is the molecular fraction of the HD in contaminated deuterium.

$$
1 /\langle\lambda\rangle=\frac{L * \rho_{D} * \sigma_{D} * N_{a v o} * c / 2}{\left\langle V_{c}\right\rangle * M_{D}}+\frac{L * \rho_{D} * \sigma_{H} * N_{a v o} *(1-c / 2)}{\left\langle V_{c}\right\rangle * M_{D}}
$$

where $N_{\text {avo }}$ is Avogadro's number. These expressions will be important for step 2.

\section{Target thickness and attenuation corrections}

The beam attenuations are different for the liquid targets since they have distinct interaction lengths and thus need to be accounted for in any target comparison analysis. The attenuation expressions, along with definitions for different target thicknesses, are as follows:

$$
\begin{gathered}
T_{D}^{D}=L * \rho_{D} *\left\langle V_{c}\right\rangle *\left[\langle D\rangle+\frac{\langle H D\rangle}{2}\right] \\
T_{H}^{D}=L * \rho_{D} *\left\langle V_{c}\right\rangle *\left[\frac{\langle H D\rangle}{2}\right] \\
T_{H}^{H}=L * \rho_{H} \\
A_{H}=\rho_{H} *\left(1-e^{-L / \lambda_{H}}\right) * 1 / L
\end{gathered}
$$




$$
A_{D}=\rho_{D} *\left(1-e^{-L /\left\langle\lambda_{D}\right\rangle}\right) * 1 / L
$$

$T_{A}^{A}$ and $A_{A}$ for solid targets are calculated in a similar way as 3.12 and 3.13. Constants averaged for the entire data set are summarized on Table 3.5.

\begin{tabular}{|c|c|}
\hline quantity & average value (weighted) \\
\hline$T_{H}^{H}$ & 3.5966 \\
\hline$T_{H}^{D}$ & 0.2437 \\
\hline$T_{D}^{D}$ & 8.0112 \\
\hline$A_{H}$ & 0.9662 \\
\hline$A_{D}$ & 0.9451 \\
\hline$V_{c}$ & 1.0056 \\
\hline$c$ & $5.91 \%$ \\
\hline
\end{tabular}

Table 3.5: Raw proton weighted average quantities for full data set.

\subsection{Cross-section ratios}

Individual targets yields are defined as follows:

$$
\begin{aligned}
\text { Yield }_{L H_{2}}= & T_{H}^{H} * N_{A} * P_{H} * A_{H} * \sigma_{p p} * \varepsilon_{H} / M_{H} \\
\text { Yield }_{L D_{2}}= & \left\langle T_{D}^{D}\right\rangle * N_{A} * P_{D} * A_{D} * \sigma_{p d} * \varepsilon_{D} / M_{D} \\
& +\left\langle T_{H}^{D}\right\rangle * N_{A} * P_{D} * A_{D} * \sigma_{p p} * \varepsilon_{D} / M_{H} \\
\text { Yield }_{A}= & T_{A}^{A} * N_{A} * P_{A} * A_{A} * \sigma_{p A} * \varepsilon_{A} / M_{A}
\end{aligned}
$$

$R_{p A}$ is the per nucleon cross-section ratio of any solid target, $A$, to $\mathrm{LD}_{2}$ and is defined as follows:

$$
R_{p A}=\frac{2}{A} \cdot \frac{\sigma_{p A}}{\sigma_{p d}}=\frac{2}{A} \frac{\left\langle T_{D}^{D}\right\rangle}{T_{A}^{A}} \frac{M_{A}}{M_{D}}\left\{\left[\frac{\frac{Y_{A}}{P_{A} * A_{A}}-\frac{Y_{N O}}{P_{N O}}}{\frac{Y_{L D 2}}{P_{D} *\left\langle A_{D}\right\rangle}-\frac{Y_{M T}}{P_{M T}}-\frac{\left\langle T_{H}^{D}\right\rangle}{T_{H}^{H}}\left[\frac{Y_{L H 2}}{P_{H} * A_{H}}-\frac{Y_{M T}}{P_{M T}}\right]}\right]\right\}
$$

Where the definitions for the variables used in Eq. 3.18 are: 
$Y_{A}$ - yield on target $X$

$T_{X}^{X}$ - thickness of $X$ in $X$ target $(\mathrm{gm} / \mathrm{cm} 2)$

$P_{X}$ - number of protons on $X$ target

$M_{X}$ - atomic mass of $X$ target $(\mathrm{LH} 2=1.008, \mathrm{LD} 2=2.014, \mathrm{C}=12.000, \mathrm{Fe}=55.845, \mathrm{~W}=183.84)$

$A_{X}$ - attenuation of protons on $X$ target

$A$ - number of nucleons on the solid target $X$

And the target subscripts refer to:

$L H 2(H)$ - liquid hydrogen

$L D 2(D)$ - liquid deuterium

$A$ - solid target of atomic mass A

NO - none

$M T$ - empty flask

\subsubsection{Statistical errors}

As defined in the previous section, the formula for cross-section for the ratio used in this analysis is as follows:

$$
R_{p A} \rightarrow R=\frac{2}{A} \cdot \frac{\sigma_{p A}}{\sigma_{p d}}=\frac{2}{A} \cdot \frac{\left\langle T_{D}^{D}\right\rangle}{T_{A}^{A}} \cdot \frac{M_{A}}{M_{D}}\left\{\left[\frac{\frac{a}{A_{A}}-d}{\frac{b}{\left\langle A_{D}\right\rangle}-c-\frac{\left\langle T_{H}^{D}\right\rangle}{T_{H}^{H}}\left[\frac{e}{A_{H}}-c\right]}\right]\right\}
$$

where $a=Y$ ield $_{A} / R A W: G 2 S E M_{A}, b=Y$ ield $_{L D 2} / R A W: G 2 S E M_{L D 2}$ and $c=Y i e l d_{M T} / R A W$ : $G 2 S E M_{M T}, d=$ Yield $_{N O} / R A W: G 2 S E M_{N O}$ and $e=Y_{\text {ield }}^{L H 2} / R A W: G 2 S E M_{L H 2}$. The 
errors, per term, are calculated as follows:

$$
\begin{aligned}
\frac{\partial R}{\partial a} & =\frac{2}{A} \cdot \frac{\left\langle T_{D}^{D}\right\rangle}{T_{A}^{A}} \cdot \frac{M_{A}}{M_{D}} \cdot \frac{1}{A_{A}} \cdot \frac{1}{\sigma_{p d}} \\
\frac{\partial R}{\partial b} & =\frac{2}{A} \cdot \frac{\left\langle T_{D}^{D}\right\rangle}{T_{A}^{A}} \cdot \frac{M_{A}}{M_{D}} \cdot \frac{1}{\left\langle A_{D}\right\rangle} \frac{\sigma_{p A}}{\sigma_{p d}^{2}} \\
\frac{\partial R}{\partial c} & =\frac{2}{A} \cdot \frac{\left\langle T_{D}^{D}\right\rangle}{T_{A}^{A}} \cdot \frac{M_{A}}{M_{D}} \cdot \frac{\sigma_{p A}}{\sigma_{p d}^{2}} \cdot\left(\frac{\left\langle T_{H}^{D}\right\rangle}{T_{H}^{H}}-1\right) \\
\frac{\partial R}{\partial d} & =\frac{2}{A} \cdot \frac{\left\langle T_{D}^{D}\right\rangle}{T_{A}^{A}} \cdot \frac{M_{A}}{M_{D}} \cdot \frac{1}{\sigma_{p d}} \\
\frac{\partial R}{\partial e} & =\frac{2}{A} \cdot \frac{\left\langle T_{D}^{D}\right\rangle}{T_{A}^{A}} \cdot \frac{M_{A}}{M_{D}} \cdot \frac{\sigma_{p A}}{\sigma_{p d}^{2}} \frac{\left\langle T_{H}^{D}\right\rangle}{T_{H}^{H}} \cdot \frac{1}{A_{H}}
\end{aligned}
$$

Errors are added in quadrature as follows:

$$
\sigma_{R}=\sqrt{\underbrace{\left(\frac{\partial R}{\partial a} \sigma_{a}\right)^{2}}_{1}+\underbrace{\left(\frac{\partial R}{\partial b} \sigma_{b}\right)^{2}}_{2}+\underbrace{\left(\frac{\partial R}{\partial c} \sigma_{c}\right)^{2}}_{3}+\underbrace{\left(\frac{\partial R}{\partial d} \sigma_{d}\right)^{2}}_{4}+\underbrace{\left(\frac{\partial R}{\partial e} \sigma_{e}\right)^{2}}_{5}}
$$

where the $\sigma_{a}, \sigma_{b}, \sigma_{c}, \sigma_{d}$ and $\sigma_{e}$ are the errors in the histograms for $a, b, c, d$ and $e$ corresponding to A, $\mathrm{LD}_{2}$, empty flask, none and $\mathrm{LH}_{2}$, respectively.

\subsection{The Intensity-Extrapolation (IE) Method}

The procedure begins by plotting the ratio of normalized cross-sections from two targets as a function of the intensity (the number of protons in the triggered bucket). We define this as:

$$
\text { intensity }=(R F+00-\text { pedestal }) * G 2 S E M /\left(Q I E_{\text {sum }}-\text { turns } * \text { buckets } * \text { pedestal }\right)
$$

The terms in Eq. 3.22 used are define as:

$R F+00$ - proportional to number of protons in a trigger bucket 
pedestal - non-zero value outputted in QIE from BIM when there are zero incoming protons. Analysis uses a value of 34 for the pedestal G2SEM - complete spill intensity sum from Secondary emission monitor (SEM) $Q I E_{\text {sum }}$ - complete spill intensity sum from Beam Intensity Monitor (BIM)

turns - there are 369,000 turns in a spill

buckets - there are 588 buckets in a turn

We should expect a flat relationship between these ratios and intensity, if we have properly dealt with all forms of rate dependence, i.e. target differences in tracking efficiencies, DAQ dead time, accidental backgrounds. If any of these remain in the data, which would manifest themselves in the form of a slope in these intensity plots, we can then exploit the idea that all forms of dependences would vanish at a beam intensity of one proton per bucket. For our measurement, we can then extract the yield ratio at the $y$-axis intercept, i.e. at a trigger intensity of zero, closely approximating the idealized value of one proton per bucket. This is done by first separating the data into different $p_{T}\left(x_{F}\right)$-bins and with a selected fitting function, one can extract the " $p_{0}$ " parameter from the fit (See Section 3.9.2). This value represents our physical yield ratio, $R_{p A}$, for a single incoming proton for that particular kinematic bin (see Section 3.9.3 and Appendix A.2 for intensity fits). The advantage of the IE method is that it is largely data driven. We require that the data, i.e. dimuon yield ratio after contamination corrections, take us to a region where the ratio of cross-sections is free from all forms of rate dependence.

\subsubsection{Logic behind fit function}

For the selection of a suitable fit function, we follow an empirical (or phenomenological) approach. A key advantage of the IE method is that all unaccounted rate and background-dependent effects will vanish at $I=0$ and thus they need not be formulated nor corrected for one-by-one. However, a complication arises when using an empirical approach in determining the best functional form for the fit. Presently, there are several candidate functions that yield similarly suitable $\chi^{2} / \mathrm{NDF}$ values 
and are thus indistinguishable. It then becomes crucial to limit them within reasonable constraints and perform a thorough systematic uncertainty analysis to account for blind spots in this selection process (See Section 4.2).

\subsubsection{Fit function for intensity dependence plots}

The fit function selected by the collaboration for ratios vs. intensity in each $p_{T}$ and $x_{F}$ bin is:

$$
F=p 0+p 1 * \text { intensity }+p 2 * \text { intensity }{ }^{2}
$$

where $p 1$ and $p 2$ are common to all $p_{T}\left(x_{F}\right)$ bins. Cross-section ratio results via the IE procedure are presented in the next chapter.

\subsubsection{Intensity dependence of $R_{p A}$}




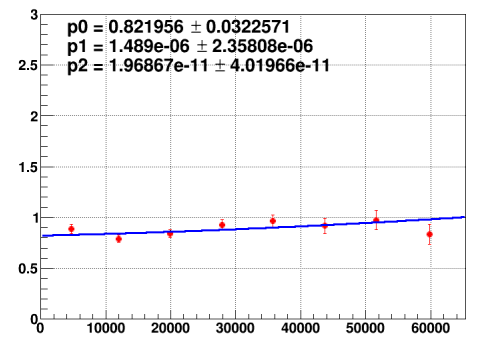

(a) $0.0<p_{T}<0.33$

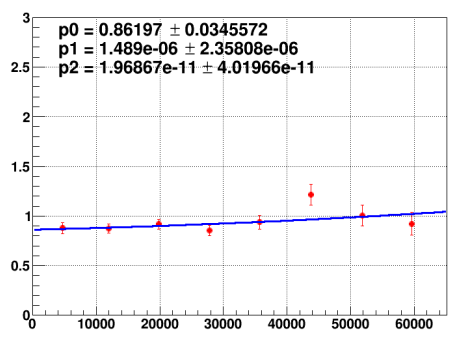

(c) $0.54<p_{T}<0.72$

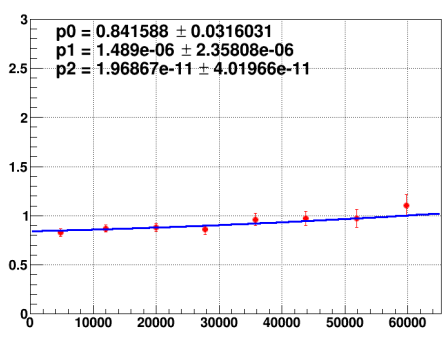

(b) $0.33<p_{T}<0.54$

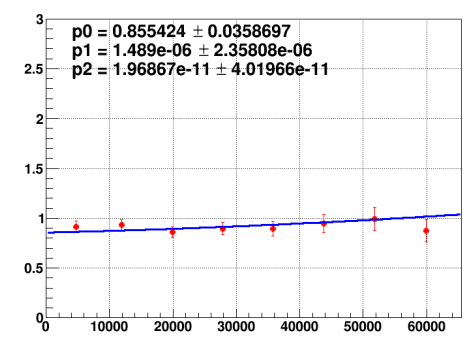

(d) $0.72<p_{T}<0.99$

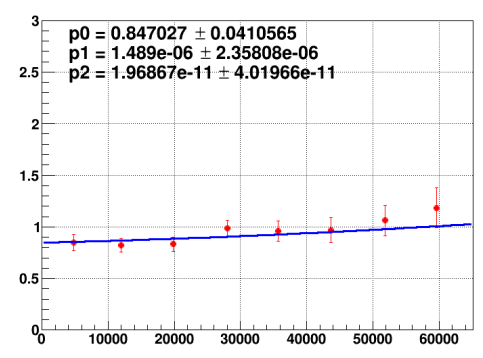

(e) $0.99<p_{T}<2.3$

Figure 3.24: Intensity dependence of $J / \psi R_{p A}$ for carbon for different $p_{T}(\mathrm{GeV} / \mathrm{c})$ bins. $F=$ $p 0+p 1 *$ intensity $+p 2 *$ intensity ${ }^{2}$ fit was used for the extrapolation, with $p 0$ taken as the nominal $R_{p A}$ value. The fit parameters $p 1$ and $p 2$ are common for all fits. Plots for iron and tungsten can be found in Appendix A.2. 


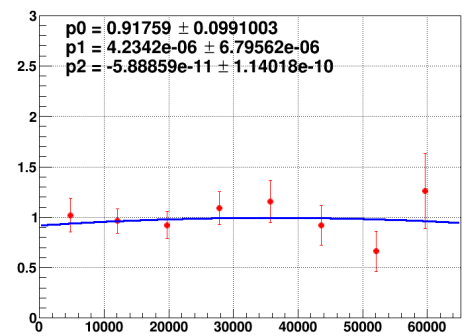

(a) $0.0<p_{T}<0.33$

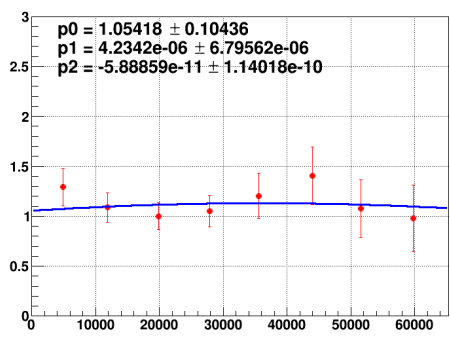

(c) $0.54<p_{T}<0.72$

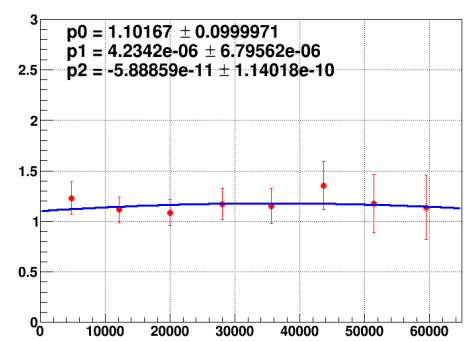

(b) $0.33<p_{T}<0.54$

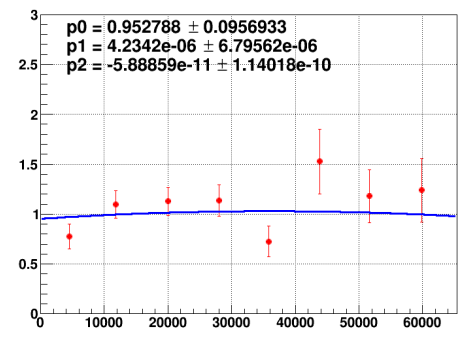

(d) $0.72<p_{T}<0.99$

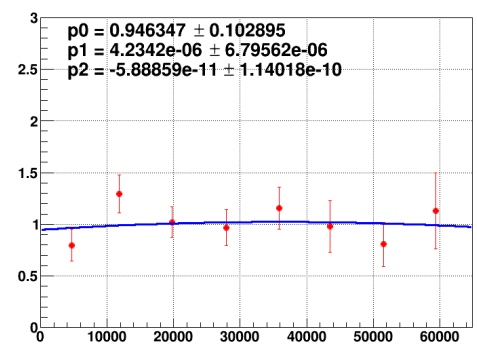

(e) $0.99<p_{T}<2.3$

Figure 3.25: Intensity dependence of DY $R_{p A}$ for carbon for different $p_{T}(\mathrm{GeV} / \mathrm{c})$ bins. $F=$ $p 0+p 1 *$ intensity $+p 2 *$ intensity ${ }^{2}$ fit was used for the extrapolation, with $p 0$ taken as the nominal $R_{p A}$ value. The fit parameters $p 1$ and $p 2$ are common for all fits. Plots for iron and tungsten can be found in Appendix A.2. 


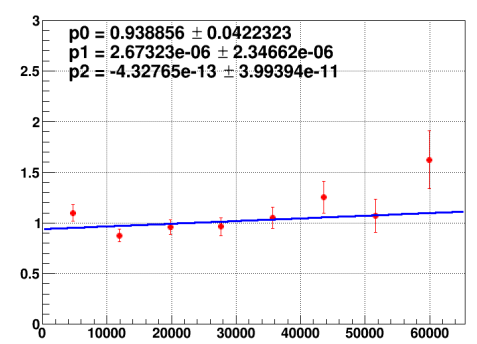

(a) $0.0<x_{F}<0.5$

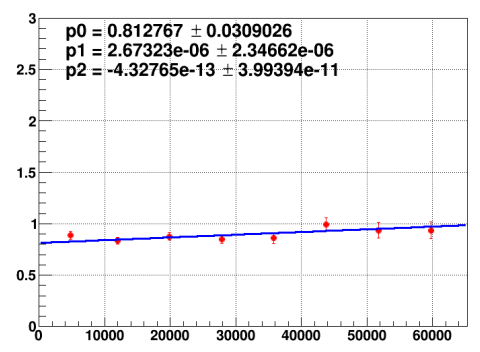

(c) $0.6<x_{F}<0.7$

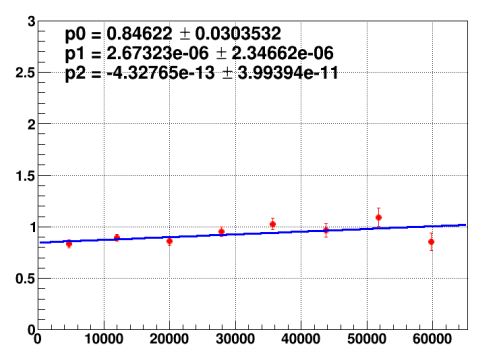

(b) $0.5<x_{F}<0.6$

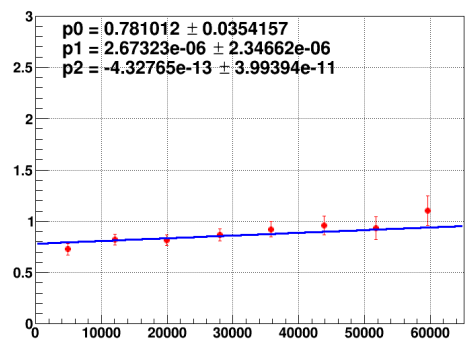

(d) $0.7<x_{F}<0.8$

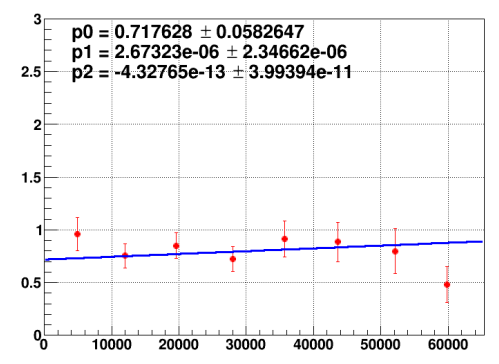

(e) $0.8<x_{F}<0.95$

Figure 3.26: $J / \psi R_{p A}$ for carbon for different $x_{F}$ bins. $F=p 0+p 1 *$ intensity $+p 2 *$ intensity $^{2}$ fit was used for the extrapolation. The fit parameters $p 1$ and $p 2$ are common for all fits. Plots for iron and tungsten can be found in Appendix A.2. 


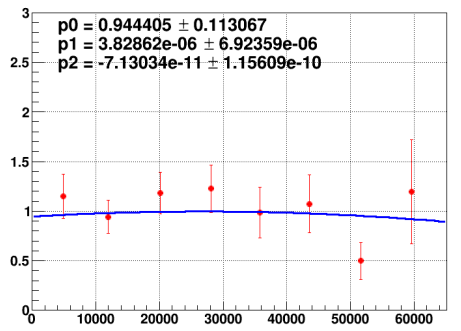

(a) $0.0<x_{F}<0.5$

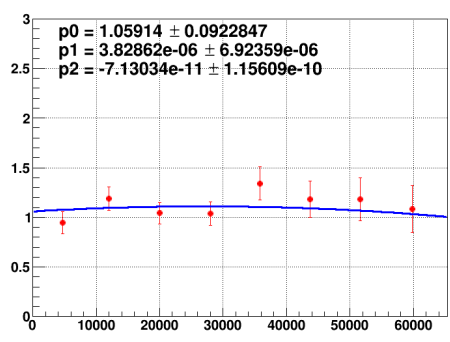

(c) $0.6<x_{F}<0.7$

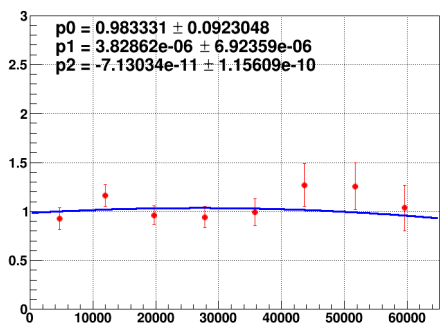

(b) $0.5<x_{F}<0.6$

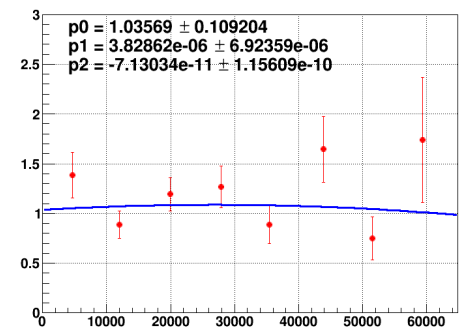

(d) $0.7<x_{F}<0.8$

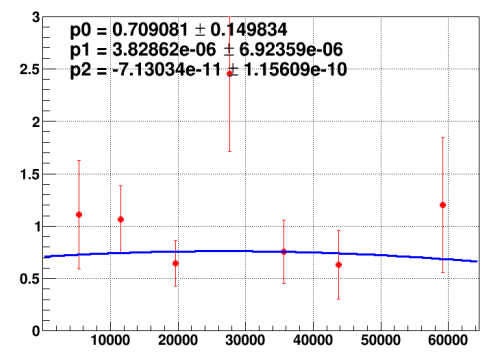

(e) $0.8<x_{F}<0.95$

Figure 3.27: DY $R_{p A}$ for carbon for different $x_{F}$ bins. $F=p 0+p 1 *$ intensity $+p 2 *$ intensity ${ }^{2}$ fit was used for the extrapolation. The fit parameters $p 1$ and $p 2$ are common for all fits. Plots for iron and tungsten can be found in Appendix A.2.

\subsection{4 kTracker efficiency corrections}

kTracker efficiency corrections are not applied to these analyses given that ratio of tracker efficiencies goes to 1 as one approaches zero intensity. This was verified in several internal studies [42]. 


\subsubsection{Physics contamination factor $F$ for $J / \psi$ signal}

The IE method is expected to properly handle background removal but it does not remove the physics background within the $J / \psi$ region in question. The amount of DY and $\Psi^{\prime}$ events (physics contamination) remaining below the $J / \psi$ peak after the analysis mass cut of $(2.7,3.2) \mathrm{GeV} / c^{2}$ still needs to be estimated for each target (see Figure 3.11). One can use the normalization values from invariant mass fits to estimate $F_{A}$, the physics contamination factor for target A define as follows:

$$
F_{A}(\%)=\frac{\left(n D Y_{A}+n P \operatorname{sip}_{A}\right)}{n D a t a_{A}} * 100
$$

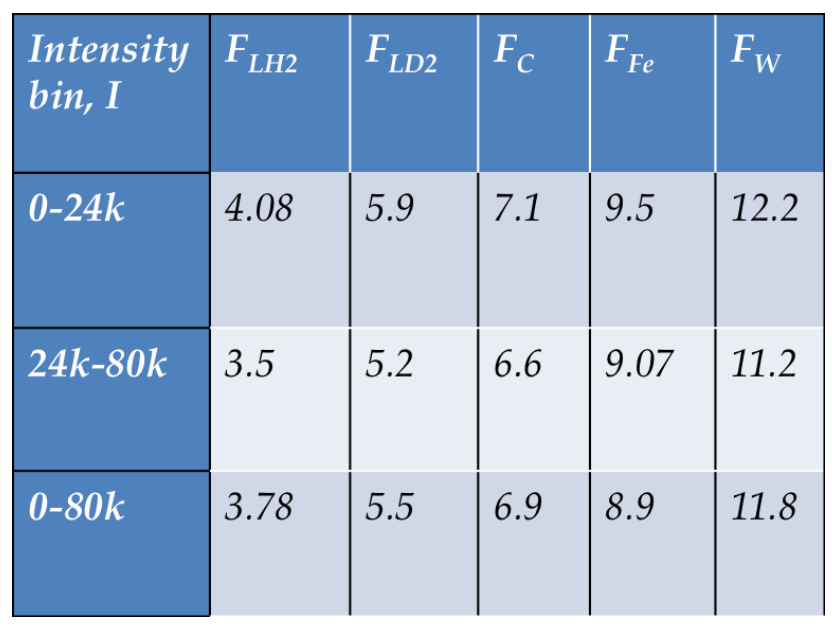

Table 3.6: $F_{A}$ factor values for each target type across different intensity bins. Values presented are percentages.

As seen in Table 3.6, $F$ seems to have a negligible intensity dependence but a strong target dependence. For $J / \psi$ ratio measurements, this additional physics background subtraction is essential and can be treated within the IE method similarly to an empty flask or "no target" subtraction. Thus, the $R_{p A}$ for $J / \psi$ ratio measurements has the form: 


$$
R_{p A}=\frac{2}{A} \frac{\left\langle T_{D}^{D}\right\rangle}{T_{A}^{A}} \frac{M_{A}}{M_{D}}\left\{\left[\frac{\frac{Y_{A}}{P_{A} * A_{A}}-\frac{F_{A} * Y_{A}}{P_{A} * A_{A}}-\frac{Y_{N O}}{P_{N O}}}{\frac{Y_{L D 2}}{P_{D} *\left\langle A_{D}\right\rangle}-\frac{F_{L D 2} * Y_{L D 2}}{P_{D} *\left\langle A_{D}\right\rangle}-\frac{Y_{M T}}{P_{M T}}-\frac{\left\langle T_{H}^{D}\right\rangle}{T_{H}^{H}}\left[\frac{Y_{L H 2}}{P_{H} * A_{H}}-\frac{F_{L H 2} * Y_{L H 2}}{P_{H} * A_{H}}-\frac{Y_{M T}}{P_{M T}}\right]}\right]\right\}
$$




\section{Chapter 4 \\ Results}

\subsection{Nuclear modification of $J / \psi$ and Drell-Yan pairs}

We define the nuclear modification ratio, $R_{p A}$, again as,

$$
R_{p A}=\frac{2}{A} \cdot \frac{\sigma_{p A}}{\sigma_{p d}}
$$

This section reports $R_{p A}$ values as a function of $p_{T}$ and $x_{F}$ for each process, along with a comparison to E866 results. A discussion of these results follows below.

\subsubsection{Discussion}

Multiple interactions of the beam parton traversing a nuclear medium can lead to broadening its transverse momentum, $p_{T}$, distribution, since each interaction is accompanied with an energy loss. With minimal final state interactions, the DY process is an ideal probe to study broadening of the transverse momentum, almost in isolation, which could give insight into the associated induced parton energy loss and could be used as a sensitive probe to constrain initial-state effects.

In contrast, the creation of a $J / \psi$ meson can be significantly influenced by the nuclear medium before and after the hard collision. Aside from the initial state effects that could generate a broadening of its transverse momentum spectrum, the production mechanism of the $J / \psi$ meson can also experience final state effects primarily in the form of nuclear absorption. A signature of nuclear absorption is a depletion in the meson yield coming from heavy nuclei to that of lighter nuclei. Figures 4.1 and 4.2 show the ratio $R_{p A}$ as a function of $p_{T}$ for $\mathrm{C} / \mathrm{LD}_{2}, \mathrm{Fe} / \mathrm{LD}_{2}$, and $\mathrm{W} / \mathrm{LD}_{2}$ for both $J / \psi$ and DY. The data shows a clear $p_{T}$ dependence for DY and a substantial suppression growing 
with solid target mass number (A) in relation to the base target $\left(L D_{2}\right)$ for $J / \psi$. We observe the following trends for each process:

$\underline{\mathrm{DY}}$

- The ratio $R_{p A}$ is consistent with unity for $\mathrm{C} / L D_{2}$.

- For iron and tungsten, there seems to be a small reduction in the per nucleon cross section ratio in the low- $p_{T}$ region coupled with an enhancement in the high- $p_{T}$ region.

- When we integrate over $p_{T}$, the ratio is 1 which is consistent with the interpretation that events at low $p_{T}$ are "pushed" to a higher $p_{T}$ value.

$\underline{J / \psi}$

- The ratio $R_{p A}$ begins to show a suppression well below unity for $\mathrm{C} / L D_{2}$.

- The ratio suppression increases for $\mathrm{Fe} / L D_{2}$ and further for $\mathrm{W} / L D_{2}$.

- A small $p_{T}$ dependence is observed for $R_{p A}=\mathrm{W} / L D_{2}$.

- A $p_{T}$ dependence for $R_{p A}=\mathrm{Fe} / L D_{2}, \mathrm{C} / L D_{2}$ is not discernible from this data.

Moreover, we compare results for the $J / \psi$ and DY process to those from the E866 experiment, which also studied $p_{T}$ dependence with an $800 \mathrm{GeV}$ proton beam and a different base target (beryllium) for ratio measurements. SeaQuest is able to provide data at a relatively lower energy (120 $\mathrm{GeV})$, as well as solid target yields relative to those from a lighter target species $\left(L D_{2}\right)($ See Figures 4.7 and 4.8). We can observe a greater suppression for both iron and tungsten ratios from the E906 data relative to the E866 data but a similar $p_{T}$ dependence across experiments for $J / \psi$ data. This increase in suppression for the E906 data can be the result of a potential energy dependence or the varying partonic contributions to $J / \psi$ production between experiments, as covered in Section 1.4. The difference could also be due to the "more disparate" target size between numerator and 
denominator for the E906 experiment. In order to disentangle this potential contribution to the increase in suppression, one could, in principle, come up with some kind of A (or A to some power) scaling to make a more direct comparison between experiments. This will be explored further by the collaboration. For DY, a slightly greater $p_{T}$ dependence for both Fe and W from E906 data relative to E866 is observed. It should also be noted that integrating $R_{p A}$ over all $p_{T}$ values gives approximately unity for both experiments.

Feynman- $x$ or $x_{F}$ is another variable of interest that sheds light on the initial state longitudinal dynamics of the interacting quark for both processes. A depletion in the $R_{p A}$ value with increasing $x_{F}$ is thought to be the result of the incoming parton experiencing energy loss due to its interaction with the cold nuclear medium

Previous experiments $[27,26]$ found a small depletion in the high $x_{F}$ region which could also be attributed to shadowing effects coming from the low- $x$ region $(\mathrm{x}<0.05$ for E866). Therefore, data outside of the shadowing region $(x>0.1)$ is essential to decouple the effects of shadowing and parton energy loss in cold nuclear matter. SeaQuest is sensitive to the region outside of the effects of shadowing and data taken on $\mathrm{C}, \mathrm{Fe}$ and $\mathrm{W}$ targets can be used to place upper limits on the energy loss of a fast parton traversing a cold nucleus. Figures 4.3 and 4.4 show the ratio $R_{p A}$ vs. $x_{F}$ for $\mathrm{C} / L D_{2}, \mathrm{Fe} / L D_{2}$ and $\mathrm{W} / L D_{2}$ for both $J / \psi$ and DY. We observe the following trends for each process:

$\underline{\mathrm{DY}}$

- The ratio $R_{p A}$ is consistent with unity for $\mathrm{C} / L D_{2}$.

- The ratio $R_{p A}$ decreases slightly with increasing $x_{F}$ for iron and tungsten.

- Since high $x_{F}$ corresponds to a low $x_{T}$, a depletion at high- $x_{F}$ could also be due to a depletion coming from the $A$ dependence of depletion in the shadowing region of the target nucleus. Hence a cut $x_{T}>0.16$ was applied to move out of the shadowing region. The results are consistent within uncertainties with and without the $x_{T}$ cut indicating that the 
effects of shadowing are negligible.

$\underline{J / \psi}$

- The ratio $R_{p A}$ decreases with increasing $x_{F}$ for all target species.

- This $x_{F}$ dependence appears to be relatively similar across target species.

- We observe a pronounced A-dependence in $J / \psi$, especially in comparison to the DY ratio results.

- This A-dependence is characterized by an increasing ratio suppression moving from carbon to tungsten.

Moreover, we compare $x_{F}$-dependence results for the $J / \psi$ and DY process to those from the E866 experiment (See Figures 4.9 and 4.10). For $J / \psi$, we observe the E906 ratio results to be systematically lower than those from E866 as a function of $x_{F}$, while they maintain a similar $x_{F}$ dependence trend. This could be due to the inverse relationship between $x_{F}$ and center of mass energy, given the lower beam energy at E906. The more disparate sized target base could also yield a greater ratio depletion for $x_{F}$ measurements, since targets that are more similar in size will experience similar parton energy loss and the effects would cancel out in a ratio measurement. For DY, trends are similar, particularly in the $x_{F}$ region of $(0.5,0.8)$. 


\subsection{Sources of systematic uncertainty}

Several sources of systematic uncertainty have been considered for this analysis:

1. Choice of fitting curve for intensity dependence, $\left(\sigma_{F i t}\right)$ : This is a significant source of systematic uncertainty. A comparison fitting function (b) is chosen and the intercepts are calculated from this functional form. The selected fit function (b) attempts to take into account a possible $p_{T}\left(\right.$ or $\left.x_{F}\right)$ dependence not factored into the results.

(a) Fitting $\mathrm{F}_{\text {main }}=\mathrm{p} 0+\mathrm{p} 1 *$ intensity $+\mathrm{p} 2{ }^{*}$ intensity $^{2}$

(b) Fitting $\mathrm{F}_{\text {sys }}=\mathrm{p} 0+\left[\mathrm{p} 10+\mathrm{p} 11 *\left(p_{T} \text { or } x_{F}\right)\right]^{*}$ intensity $+\left[\mathrm{p} 20+\mathrm{p} 21 *\left(p_{T} \text { or } x_{F}\right)\right]^{*}$ intensity ${ }^{2}$

The difference in intercepts between our main fit function (a) and the selected comparison fit (b) were estimated and used as the systematic uncertainty contribution due to fit function choice (all parameters except for $p 0$ are common to all bins in both fits).

2. Contamination of $\psi$ ' and DY process in the $J / \psi$ mass region, $\left(\sigma_{F}\right)$ : This is also a large source of systematic uncertainty. We estimated the $\psi$ ' and DY process contamination (physics contamination) in the $J / \psi$ mass region using mass fits. There is a notable difference in physics contamination here between target types making it important to introduce a physics contamination factor, $F$, (see Section 3.9.5). As a conservative choice, $F$ was doubled and halved for all targets and the intercepts were calculated using each, respectively. The difference between these intercepts to the intercepts using the derived $F$ factor were estimated and used as the lower and upper bounds of the systematic uncertainty contribution due to this contamination factor.

3. Contamination of the $J / \psi$ and $\psi$ ' tail into the Drell-Yan mass region: The contamination of $J / \psi$ and $\psi^{\prime}$ in the DY mass region for $L D_{2}$ for the highest (most contaminated) $p_{T}$ bin is less than $1 \%$ and for tungsten for the highest (most contaminated) $p_{T}$ bin is less than $0.5 \%$. The contamination of $J / \psi$ and $\psi$ ' in the DY mass region for $L D_{2}$ for the most contaminated 
$x_{F}$ bin is less than $0.7 \%$ and for tungsten for the most contaminated $x_{F}$ bin is less than 0.5 $\%$. Therefore, systematics due to this source can be deemed negligible.

4. Deuterium contamination: The deuterium purity is $95.8 \pm 0.2 \%$. Two sets of intercepts were calculated. One with $95.8+0.2$ and another with $95.8-0.2$ and the change in the intercept values was negligible as the difference was below $0.5 \%$.

5. Target Length difference between $\mathbf{L D}_{2}$ and $\mathbf{L} \mathbf{H}_{2}$ flasks: It was mentioned in [28] that the uncertainty in the difference between flask lengths is $0.2 \%$. Two sets of intercepts were calculated with $50.8+0.002$ and $50.8-0.002$ and the change is negligible as the difference was below $0.5 \%$.

6. Accuracy of raw protons (= G2SEM): The fluctuation in the G2SEM/QIEsum ratio was observed to be $0.16 \%$. So we adjusted the trigger intensity values to be $+0.16 \%$ and -0.16 $\%$ and calculated the fit parameters. The changes in the intercepts are negligible as the difference was below $0.5 \%$.

7. Varying the QIE pedestal: When the G2SEM value is zero the QIE readout shows the QIE pedestal value. This value is used to calculate our trigger intensity, I, variable and has been shown to vary. A nominal value of 34 was used with a \pm 4 uncertainty. The difference between the nominal value intercepts and high/low value pedestal intercepts were calculated and the change was observed to be less than $0.5 \%$ thus contributing negligibly to the systematic uncertainty.

8. Overall proton normalization, $\left(\sigma_{p-n o r m}\right)$ : A $5 \%$ additional normalization has been considered to account for the difference in results when normalizing with raw protons instead of live protons.

9. Beam loss on solid target, $\left(\sigma_{\text {beam-loss }}\right)$ : A $0.5 \%$ additional normalization has been considered to account for the beam loss on the solid target due to a $1.6 \mathrm{~cm}$ offset. 
The various contributions to the lower and upper bounds of the total systematic error estimation $\left(\sigma_{\text {total-low }}, \sigma_{\text {total-up }}\right)$ were added in quadrature, individually, as follows:

$$
\begin{aligned}
& \sigma_{\text {total-low }}=\sqrt{\sum_{i}\left(\sigma_{i-l o w}\right)^{2}} \\
& \sigma_{\text {total-up }}=\sqrt{\sum_{i}\left(\sigma_{i-\text { up }}\right)^{2}}
\end{aligned}
$$

The non-negligible systematic error contributions and totals are presented in Tables 4.1 - 4.6 for $p_{T}$ and Tables $4.7-4.12$ for $x_{F}$. 


\subsection{1 $p_{T}$ systematic error for $J / \psi$ and Drell-Yan processes}

\begin{tabular}{|c|c|c|c|c|c|c|c|c|}
\hline$p T$ & $\sigma$ Fit-low & $\sigma$ Fit-up & $\sigma$ F-low & $\sigma F-u p$ & $\sigma$ p-norm (low/up) & Obeam-loss (low/up) & $\sigma$ total-low & $\sigma$ total-up \\
\hline $0-0.33$ & 0.0100 & 0.0000 & 0.0149 & 0.0067 & 0.0411 & 0.0041 & 0.0450 & 0.0418 \\
\hline $0.33-0.54$ & 0.0036 & 0.0000 & 0.0153 & 0.007 & 0.0421 & 0.0042 & 0.0451 & 0.0429 \\
\hline $0.54-0.72$ & 0.0000 & 0.0019 & 0.0151 & 0.0068 & 0.0431 & 0.0043 & 0.0459 & 0.0439 \\
\hline $0.72-0.99$ & 0.0000 & 0.0079 & 0.0156 & 0.007 & 0.0428 & 0.0043 & 0.0457 & 0.0443 \\
\hline $0.99-2.3$ & 0.0000 & 0.0198 & 0.0156 & 0.0071 & 0.0424 & 0.0042 & 0.0453 & 0.0475 \\
\hline
\end{tabular}

Table 4.1: $J / \psi R_{p A}$ systematic error per $p_{T}$ bin $(\mathrm{GeV} / \mathrm{c})$ for carbon

\begin{tabular}{|c|c|c|c|c|c|c|c|c|}
\hline$p T$ & $\sigma$ Fit-low & $\sigma$ Fit-up & $\sigma$ F-low & $\sigma F-u p$ & $\sigma$ p-norm (low/up) & Obeam-loss (low/up) & $\sigma$ total-low & $\sigma$ total-up \\
\hline $0-0.33$ & 0.0000 & 0.0483 & 0.0254 & 0.0115 & 0.0292 & 0.0029 & 0.0388 & 0.0577 \\
\hline $0.33-0.54$ & 0.0000 & 0.0168 & 0.0256 & 0.0116 & 0.0290 & 0.0029 & 0.0388 & 0.0356 \\
\hline $0.54-0.72$ & 0.0102 & 0.0000 & 0.0263 & 0.0119 & 0.0303 & 0.0030 & 0.0415 & 0.0327 \\
\hline $0.72-0.99$ & 0.0393 & 0.0000 & 0.0263 & 0.012 & 0.0299 & 0.0030 & 0.0560 & 0.0323 \\
\hline $0.99-2.3$ & 0.0924 & 0.0000 & 0.0286 & 0.0131 & 0.0328 & 0.0033 & 0.1022 & 0.0355 \\
\hline
\end{tabular}

Table 4.2: $J / \psi R_{p A}$ systematic error per $p_{T}$ bin $(\mathrm{GeV} / \mathrm{c})$ for iron

\begin{tabular}{|l|r|r|r|r|r|r|r|r|}
\hline$p T$ & $\sigma$ Fit-low & $\sigma$ Fit-up & $\sigma$ F-low & $\sigma$ F-up & $\sigma$ p-norm (low/up) & б beam-loss (low/up) & $\sigma$ total-low & $\sigma$ total-up \\
\hline $0-0.33$ & 0.0000 & 0.0151 & 0.0317 & 0.0145 & 0.0195 & 0.0019 & 0.0372 & 0.0286 \\
\hline $0.33-0.54$ & 0.0000 & 0.0043 & 0.0334 & 0.0153 & 0.0205 & 0.0020 & 0.0392 & 0.0260 \\
\hline $0.54-0.72$ & 0.0045 & 0.0000 & 0.0358 & 0.0164 & 0.0222 & 0.0022 & 0.0424 & 0.0277 \\
\hline $0.72-0.99$ & 0.0144 & 0.0000 & 0.0374 & 0.0171 & 0.0230 & 0.0023 & 0.0463 & 0.0287 \\
\hline $0.99-2.3$ & 0.0323 & 0.0000 & 0.0406 & 0.0185 & 0.0250 & 0.0025 & 0.0576 & 0.0312 \\
\hline
\end{tabular}

Table 4.3: $J / \psi R_{p A}$ systematic error per $p_{T}$ bin $(\mathrm{GeV} / \mathrm{c})$ for tungsten 


\begin{tabular}{|l|r|r|r|r|r|r|}
\hline$p T$ & $\sigma$ Fit-low & $\sigma$ Fit-up & $\sigma$ p-norm (low/up) & б beam-loss (low/up) & $\sigma$ total-low & $\sigma$ total-up \\
\hline $0-0.33$ & 0.0000 & 0.0913 & 0.0459 & 0.0046 & 0.0461 & 0.1023 \\
\hline $0.33-0.54$ & 0.0000 & 0.0486 & 0.0551 & 0.0055 & 0.0554 & 0.0736 \\
\hline $0.54-0.72$ & 0.0000 & 0.0112 & 0.0527 & 0.0053 & 0.0530 & 0.0541 \\
\hline $0.72-0.99$ & 0.0294 & 0.0000 & 0.0476 & 0.0048 & 0.0562 & 0.0479 \\
\hline $0.99-2.3$ & 0.1048 & 0.0000 & 0.0473 & 0.0047 & 0.1151 & 0.0476 \\
\hline
\end{tabular}

Table 4.4: DY $R_{p A}$ systematic error per $p_{T}$ bin $(\mathrm{GeV} / \mathrm{c})$ for carbon

\begin{tabular}{|c|c|c|c|c|c|c|}
\hline$p T$ & $\sigma$ Fit-low & $\sigma$ Fit-up & $\sigma$ p-norm (low/up) & б beam-loss (low/up) & $\sigma$ total-low & $\sigma$ total-up \\
\hline $0-0.33$ & 0.0000 & 0.0280 & 0.0436 & 0.0044 & 0.0438 & 0.0520 \\
\hline $0.33-0.54$ & 0.0000 & 0.0062 & 0.0490 & 0.0049 & 0.0492 & 0.0496 \\
\hline $0.54-0.72$ & 0.0028 & 0.0000 & 0.0515 & 0.0051 & 0.0518 & 0.0517 \\
\hline $0.72-0.99$ & 0.0101 & 0.0000 & 0.0570 & 0.0057 & 0.0582 & 0.0573 \\
\hline \begin{tabular}{|l|}
$0.99-2.3$ \\
\end{tabular} & 0.0373 & 0.0000 & 0.0584 & 0.0058 & 0.0695 & 0.0587 \\
\hline
\end{tabular}

Table 4.5: DY $R_{p A}$ systematic error per $p_{T}$ bin $(\mathrm{GeV} / \mathrm{c})$ for iron

\begin{tabular}{|c|c|c|c|c|c|c|}
\hline$p T$ & $\sigma$ Fit-low & $\sigma$ Fit-up & $\sigma$ p-norm (low/up) & б beam-loss (low/up) & $\sigma$ total-low & $\sigma$ total-up \\
\hline $0-0.33$ & 0.0320 & 0.0000 & 0.0428 & 0.0043 & 0.0536 & 0.0430 \\
\hline $0.33-0.54$ & 0.0144 & 0.0000 & 0.0427 & 0.0043 & 0.0453 & 0.0429 \\
\hline $0.54-0.72$ & 0.0000 & 0.0003 & 0.0502 & 0.0050 & 0.0505 & 0.0505 \\
\hline $0.72-0.99$ & 0.0000 & 0.0192 & 0.0549 & 0.0055 & 0.0552 & 0.0584 \\
\hline $\mid 0.99-2.3$ & 0.0000 & 0.0558 & 0.0578 & 0.0058 & 0.0581 & 0.0805 \\
\hline
\end{tabular}

Table 4.6: DY $R_{p A}$ systematic error per $p_{T}$ bin $(\mathrm{GeV} / \mathrm{c})$ for tungsten 


\subsection{2 $x_{F}$ systematic error for $J / \psi$ and Drell-Yan processes}

\begin{tabular}{|l|r|r|r|r|r|r|r|r|}
\hline $\boldsymbol{x F}$ & $\sigma$ Fit-low & $\sigma$ Fit-up & \multicolumn{1}{|c|}{$\sigma$ F-low } & $\sigma$ F-up & $\sigma$ p-norm (low/up) & $\sigma$ beam-loss (low/up) & $\sigma$ total-low & $\sigma$ total-up \\
\hline $0-0.5$ & 0.0000 & 0.0189 & 0.0166 & 0.0076 & 0.0469 & 0.0047 & 0.0500 & 0.0514 \\
\hline $0.5-0.6$ & 0.0000 & 0.0078 & 0.0153 & 0.0070 & 0.0423 & 0.0042 & 0.0452 & 0.0438 \\
\hline $0.6-0.7$ & 0.0014 & 0.0000 & 0.0148 & 0.0068 & 0.0406 & 0.0041 & 0.0435 & 0.0414 \\
\hline $0.7-0.8$ & 0.0107 & 0.0000 & 0.0145 & 0.0066 & 0.0391 & 0.0039 & 0.0432 & 0.0398 \\
\hline $0.8-0.95$ & 0.0122 & 0.0000 & 0.0139 & 0.0064 & 0.0359 & 0.0036 & 0.0405 & 0.0366 \\
\hline
\end{tabular}

Table 4.7: $J / \psi R_{p A}$ systematic error per $x_{F}$ bin for carbon

\begin{tabular}{|l|r|r|r|r|r|r|r|r|}
\hline$x F$ & $\sigma$ Fit-low & $\sigma$ Fit-up & $\sigma$ F-low & $\sigma$ F-up & $\sigma$ p-norm (low/up) & $\sigma$ beam-loss (low/up) & $\sigma$ total-low & $\sigma$ total-up \\
\hline $0-0.5$ & 0.0297 & 0.0000 & 0.0290 & 0.0132 & 0.0340 & 0.0034 & 0.0537 & 0.0366 \\
\hline $0.5-0.6$ & 0.0132 & 0.0000 & 0.0272 & 0.0124 & 0.0313 & 0.0031 & 0.0436 & 0.0338 \\
\hline $0.6-0.7$ & 0.0000 & 0.0056 & 0.0248 & 0.0113 & 0.0282 & 0.0028 & 0.0376 & 0.0310 \\
\hline $0.7-0.8$ & 0.0000 & 0.0254 & 0.0244 & 0.0111 & 0.0274 & 0.0027 & 0.0368 & 0.0391 \\
\hline $0.8-0.95$ & 0.0000 & 0.0497 & 0.0219 & 0.0100 & 0.0243 & 0.0024 & 0.0328 & 0.0562 \\
\hline
\end{tabular}

Table 4.8: $J / \psi R_{p A}$ systematic error per $x_{F}$ bin for iron

\begin{tabular}{|l|r|r|r|r|r|r|r|r|}
\hline$x F$ & $\sigma$ Fit-low & $\sigma$ Fit-up & $\sigma$ F-low & $\sigma$ F-up & $\sigma$ p-norm (low/up) & б beam-loss (low/up) & $\sigma$ total-low & $\sigma$ total-up \\
\hline $0-0.5$ & 0.0387 & 0.0000 & 0.0413 & 0.0189 & 0.0256 & 0.0026 & 0.0621 & 0.0320 \\
\hline $0.5-0.6$ & 0.0174 & 0.0000 & 0.0369 & 0.0168 & 0.0228 & 0.0023 & 0.0468 & 0.0284 \\
\hline $0.6-0.7$ & 0.0000 & 0.0052 & 0.0318 & 0.0146 & 0.0195 & 0.0019 & 0.0373 & 0.0250 \\
\hline $0.7-0.8$ & 0.0000 & 0.0288 & 0.0323 & 0.0147 & 0.0198 & 0.0020 & 0.0380 & 0.0380 \\
\hline $0.8-0.95$ & 0.0000 & 0.0568 & 0.0222 & 0.0103 & 0.0133 & 0.0013 & 0.0259 & 0.0592 \\
\hline
\end{tabular}

Table 4.9: $J / \psi R_{p A}$ systematic error per $x_{F}$ bin for tungsten 


\begin{tabular}{|l|r|r|r|r|r|r|}
\hline$x F$ & $\sigma$ Fit-low & $\sigma$ Fit-up & $\sigma$ p-norm (low/up) & $\sigma$ beam-loss (low/up) & $\sigma$ total-low & $\sigma$ total-up \\
\hline $0-0.5$ & 0.0226 & 0.0000 & 0.0472 & 0.0047 & 0.0526 & 0.0475 \\
\hline $0.5-0.6$ & 0.0102 & 0.0000 & 0.0492 & 0.0049 & 0.0504 & 0.0494 \\
\hline $0.6-0.7$ & 0.0000 & 0.0066 & 0.0530 & 0.0053 & 0.0532 & 0.0536 \\
\hline $0.7-0.8$ & 0.0000 & 0.0244 & 0.0518 & 0.0052 & 0.0520 & 0.0575 \\
\hline $0.8-0.95$ & 0.0000 & 0.0428 & 0.0355 & 0.0035 & 0.0356 & 0.0557 \\
\hline
\end{tabular}

Table 4.10: DY $R_{p A}$ systematic error per $x_{F}$ bin for carbon

\begin{tabular}{|l|r|r|r|r|r|r|}
\hline$x F$ & $\sigma$ Fit-low & $\sigma$ Fit-up & $\sigma$ p-norm (low/up) & $\sigma$ beam-loss (low/up) & $\sigma$ total-low & $\sigma$ total-up \\
\hline $0-0.5$ & 0.1035 & 0.0000 & 0.0567 & 0.0057 & 0.1181 & 0.0570 \\
\hline $0.5-0.6$ & 0.0425 & 0.0000 & 0.0534 & 0.0053 & 0.0684 & 0.0537 \\
\hline $0.6-0.7$ & 0.0000 & 0.0205 & 0.0480 & 0.0048 & 0.0483 & 0.0524 \\
\hline $0.7-0.8$ & 0.0000 & 0.0907 & 0.0489 & 0.0049 & 0.0491 & 0.1032 \\
\hline $0.8-0.95$ & 0.0000 & 0.1890 & 0.0276 & 0.0028 & 0.0278 & 0.1910 \\
\hline
\end{tabular}

Table 4.11: DY $R_{p A}$ systematic error per $x_{F}$ bin for iron

\begin{tabular}{|l|r|r|r|r|r|r|}
\hline$x F$ & $\sigma$ Fit-low & $\sigma$ Fit-up & $\sigma$ p-norm (low/up) & $\sigma$ beam-loss (low/up) & $\sigma$ total-low & $\sigma$ total-up \\
\hline $0-0.5$ & 0.1286 & 0.0000 & 0.0574 & 0.0057 & 0.1409 & 0.0577 \\
\hline $0.5-0.6$ & 0.0603 & 0.0000 & 0.0527 & 0.0053 & 0.0802 & 0.0530 \\
\hline $0.6-0.7$ & 0.0000 & 0.0221 & 0.0459 & 0.0046 & 0.0461 & 0.0511 \\
\hline $0.7-0.8$ & 0.0000 & 0.1097 & 0.0483 & 0.0048 & 0.0486 & 0.1200 \\
\hline $0.8-0.95$ & 0.0000 & 0.2223 & 0.0425 & 0.0043 & 0.0427 & 0.2263 \\
\hline
\end{tabular}

Table 4.12: DY $R_{p A}$ systematic error per $x_{F}$ bin for tungsten 


\section{3 $p_{T}$ dependence}

4.3.1 $p_{T}$ dependence results for $J / \psi$
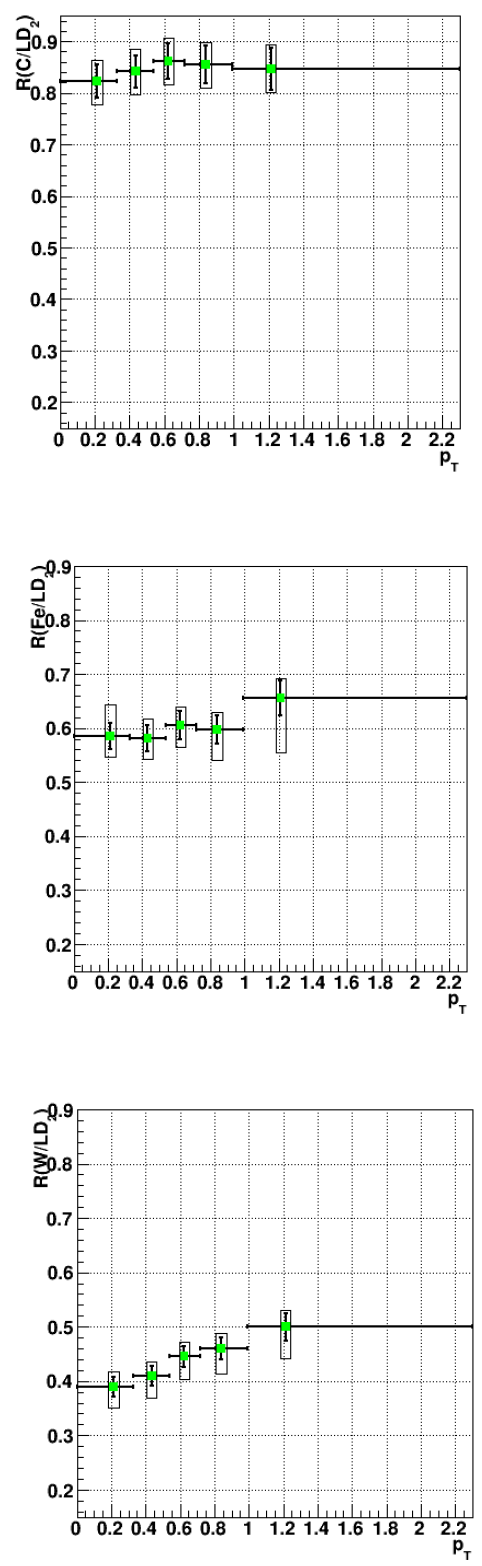

Figure 4.1: $R_{p A}$ vs $p_{T}(\mathrm{GeV} / \mathrm{c})$ for carbon, iron and tungsten for $J / \psi$. Boxes around points represent systematic error. 


\subsection{2 $p_{T}$ dependence results for Drell-Yan}
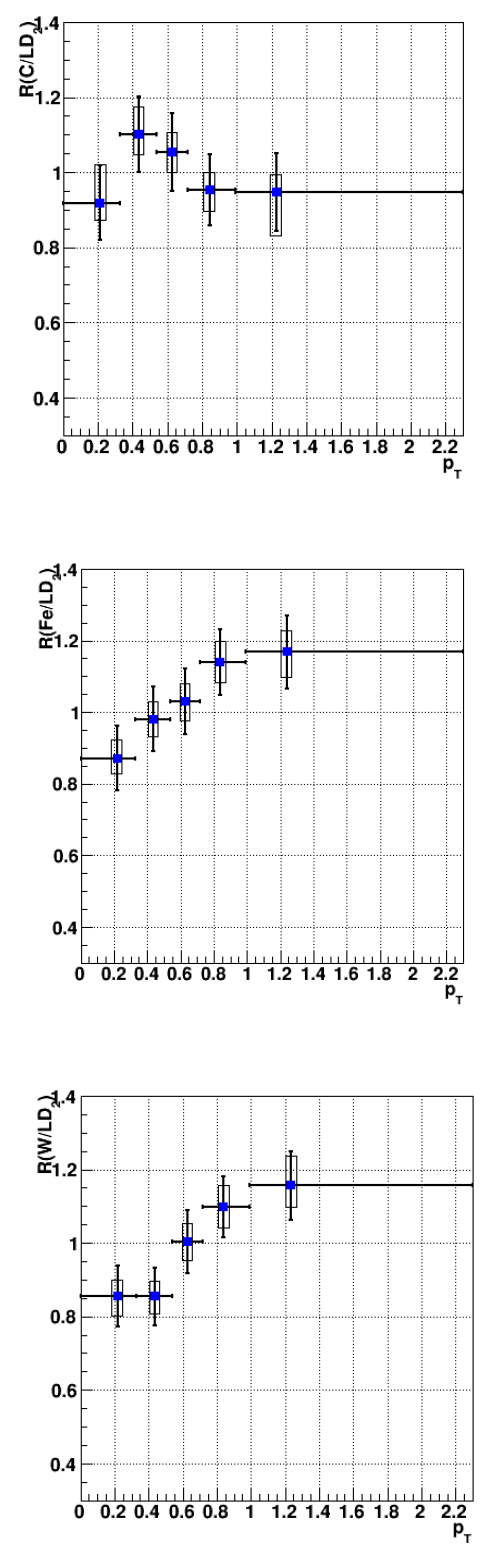

Figure 4.2: $R_{p A}$ vs $p_{T}(\mathrm{GeV} / \mathrm{c})$ for carbon, iron and tungsten for DY. Boxes around points represent systematic error. 


\section{4 $x_{F}$ dependence}

4.4.1 $x_{F}$ dependence results for $J / \psi$
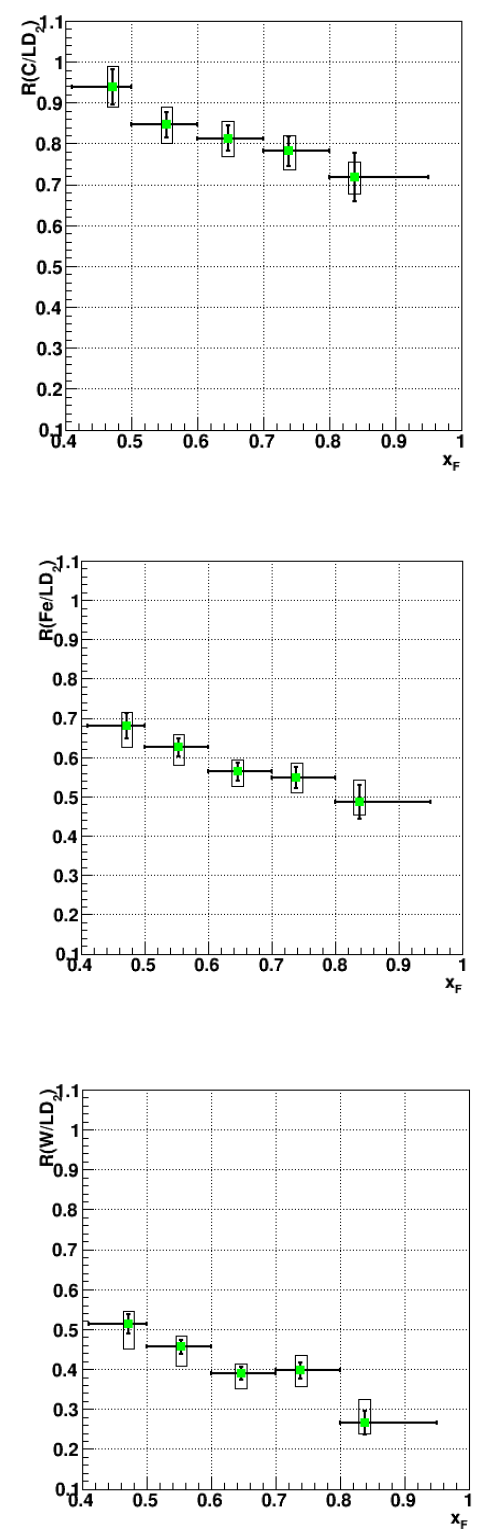

Figure 4.3: $R_{p A}$ vs $x_{F}$ for carbon, iron and tungsten for $J / \psi$. Boxes around points represent systematic error. 
4.4.2 $x_{F}$ dependence results for Drell-Yan
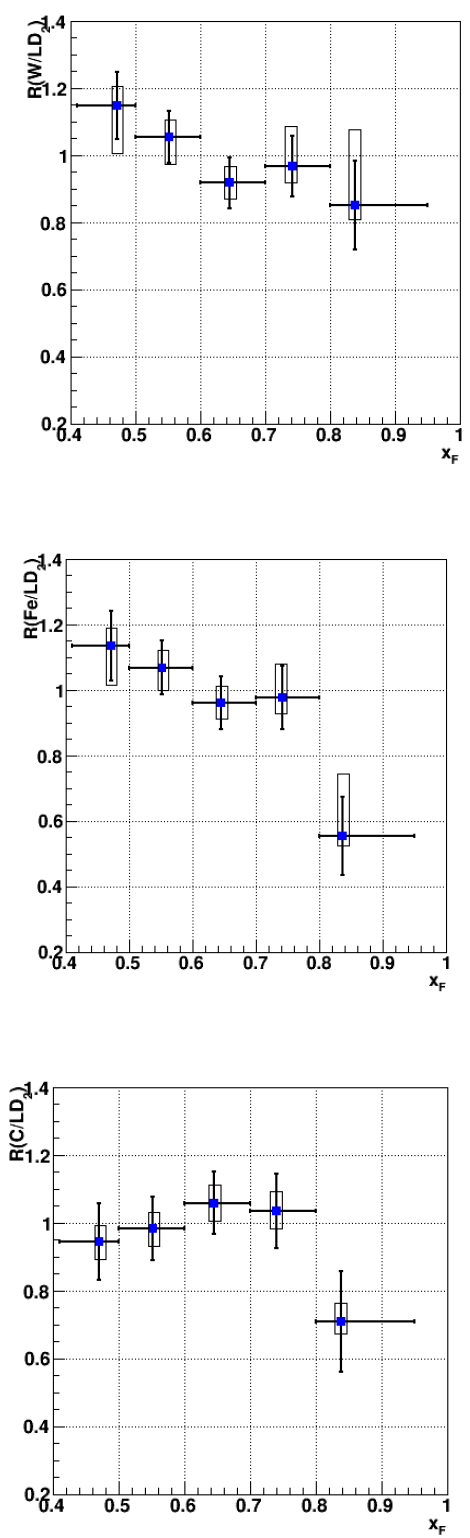

Figure 4.4: $R_{p A}$ vs $x_{F}$ for carbon, iron and tungsten for DY. Boxes around points represent systematic error.

\subsection{Nuclear dependence comparisons}



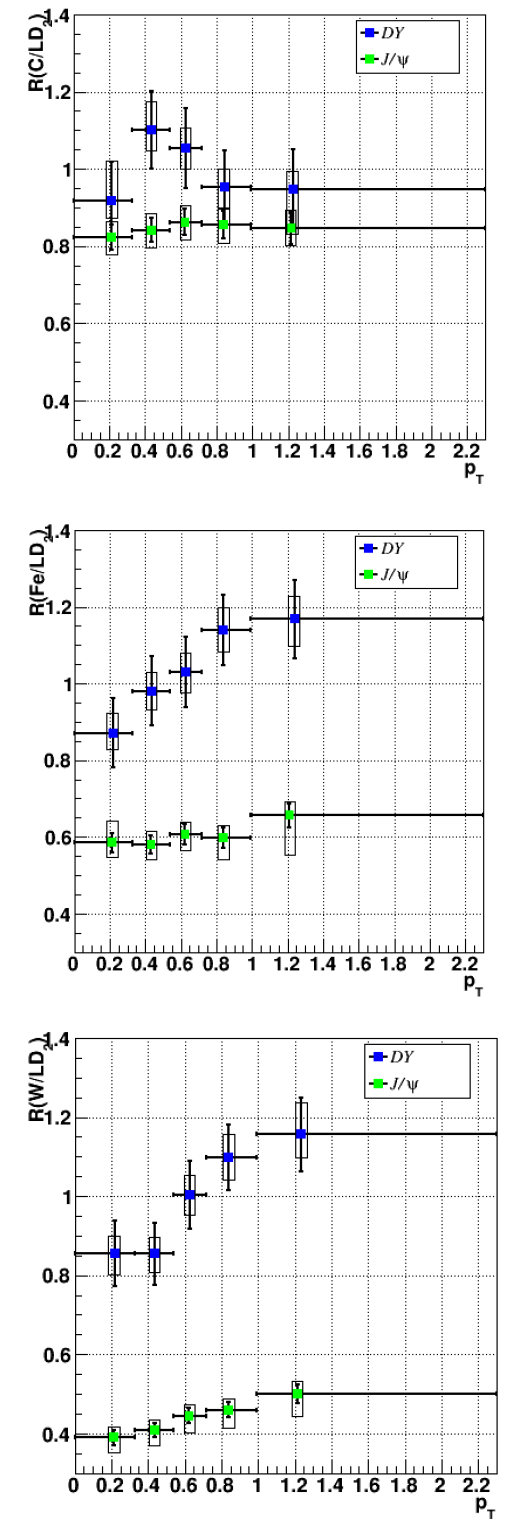

Figure 4.5: $R_{p A}$ vs $p_{T}(\mathrm{GeV} / \mathrm{c})$ for carbon, iron and tungsten for both $J / \psi$ and DY. Boxes around points represent systematic error. 

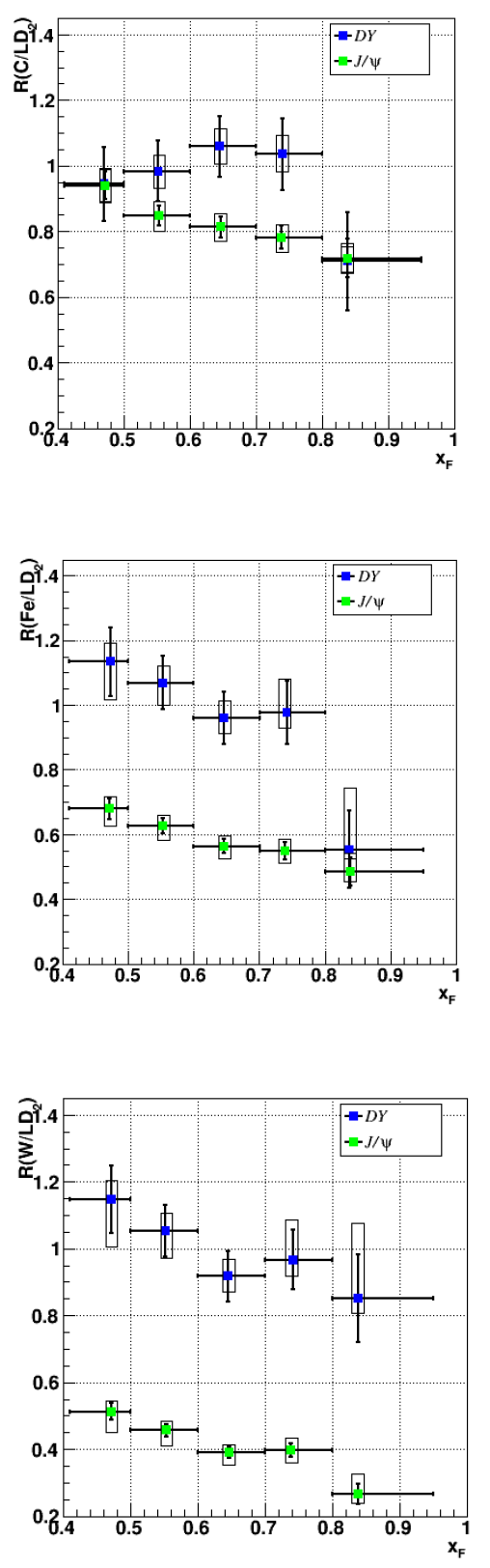

Figure 4.6: $R_{p A}$ vs $x_{F}$ for carbon, iron and tungsten for both $J / \psi$ and DY. Boxes around points represent systematic error. 

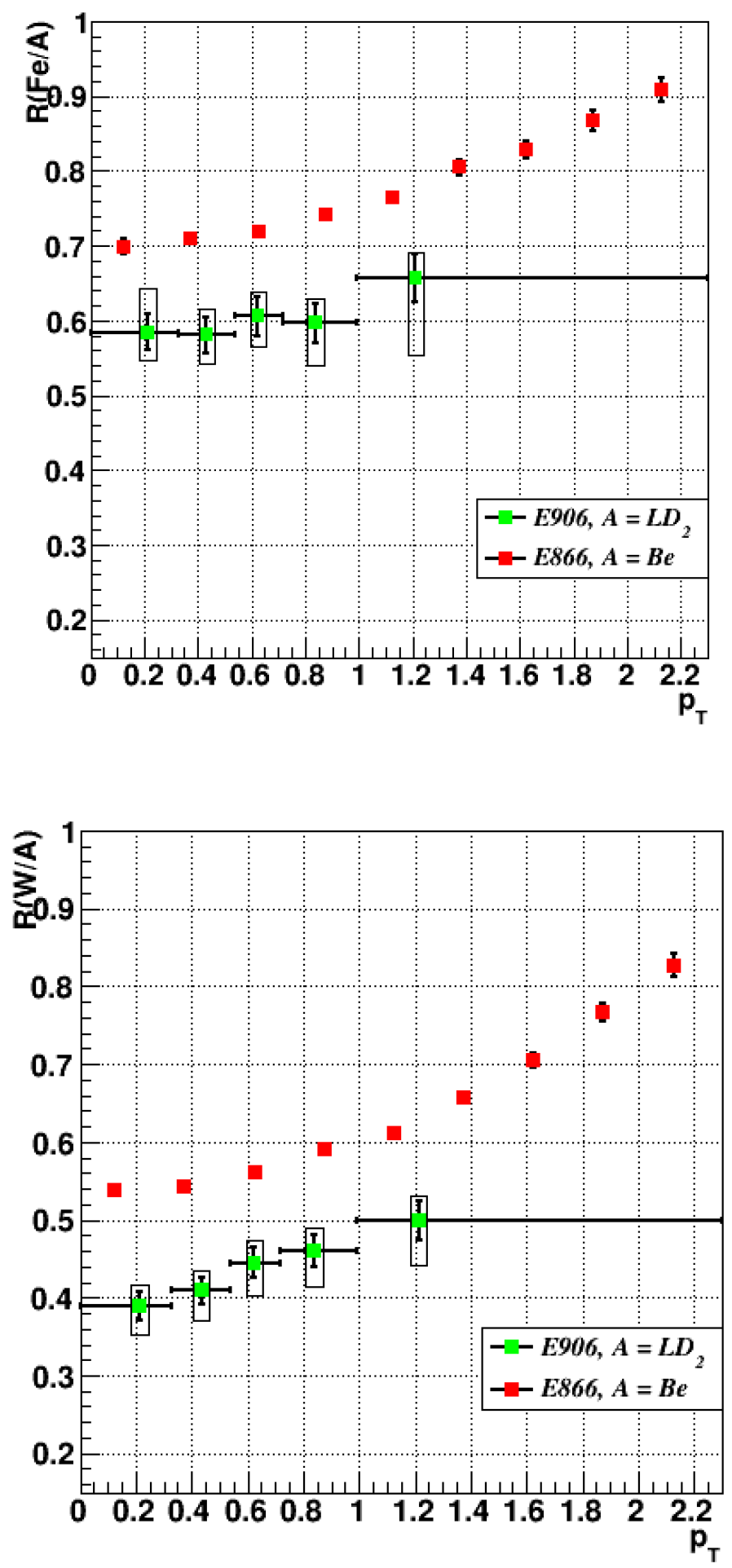

Figure 4.7: $J / \psi R_{p A}$ vs $p_{T}(\mathrm{GeV} / \mathrm{c})$ for iron and tungsten as measured in E906 and E866. Liquid deuterium $\left(L D_{2}\right)$ was the base target for $\mathrm{E} 906$ ratio measurements, while Beryllium $(\mathrm{Be})$ was the base target for E866 ratio measurements. Boxes around points represent systematic error for E906. E866 reported a systematic error of $2 \%$ across all $p_{T}$ bins [25]. 

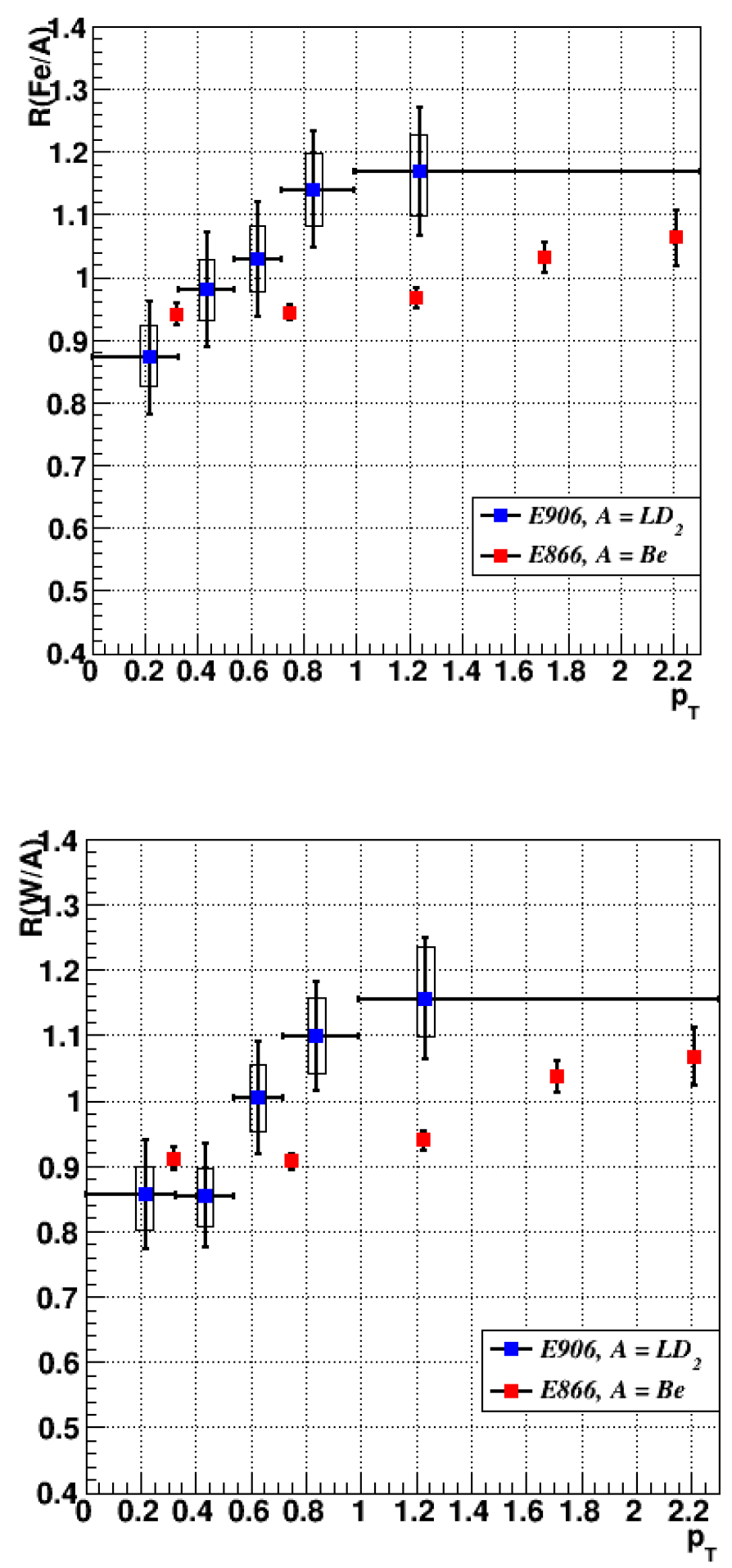

Figure 4.8: DY $R_{p A}$ vs $p_{T}(\mathrm{GeV} / \mathrm{c})$ for iron and tungsten as measured in E906 and E866. Liquid deuterium $\left(L D_{2}\right)$ was the base target for E906 ratio measurements, while Beryllium (Be) was the base target for E866 ratio measurements. Boxes around points represent systematic error for E906. E866 reported a systematic error of $1 \%$ across all $p_{T}$ bins [27]. 

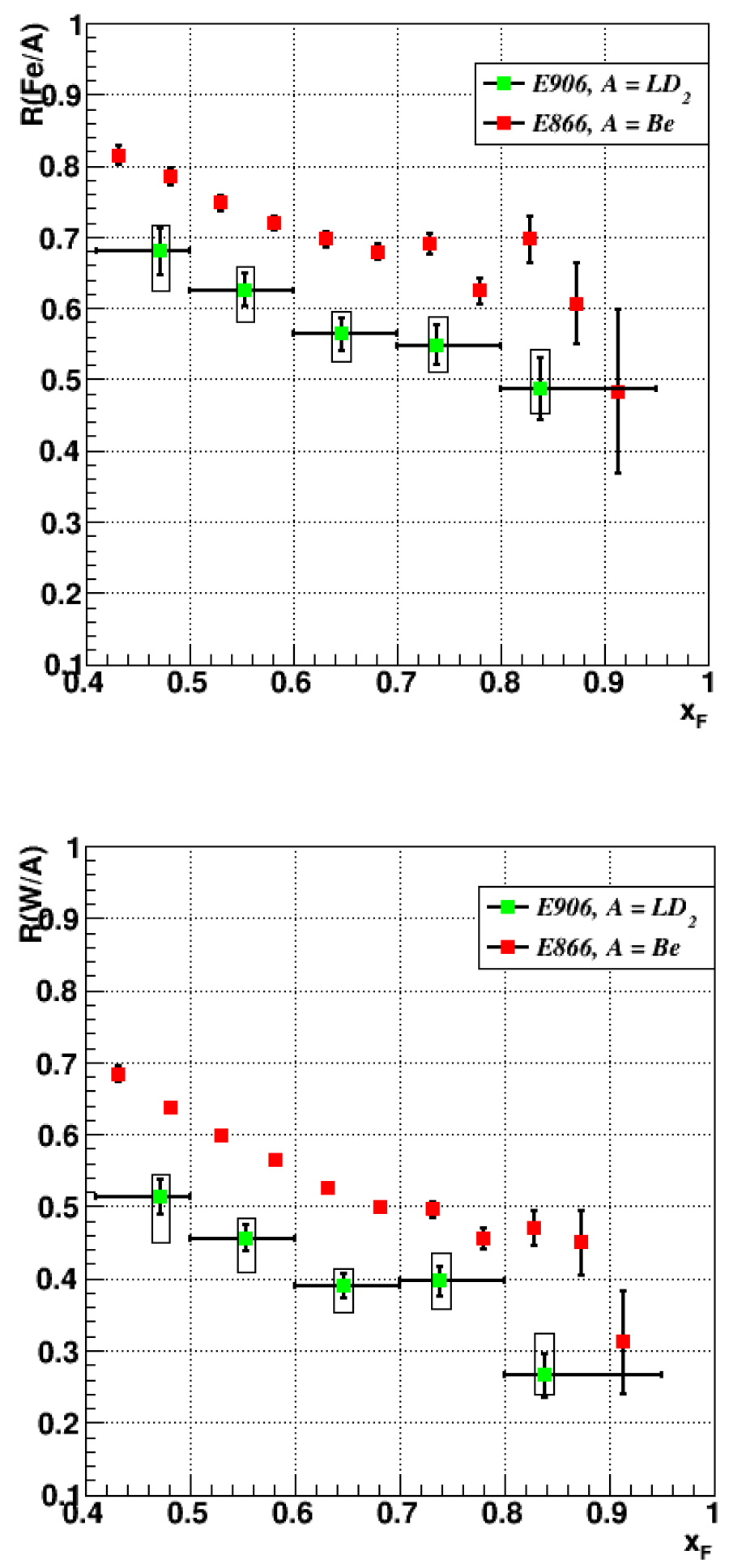

Figure 4.9: $J / \psi R_{p A}$ vs $x_{F}$ for iron and tungsten as measured in E906 and E866. Liquid deuterium $\left(L D_{2}\right)$ was the base target for E906 ratio measurements, while Beryllium $(\mathrm{Be})$ was the base target for E866 ratio measurements. Boxes around points represent systematic error for E906. E866 reported a systematic error of $3 \%$ across all $x_{F}$ bins [25]. 

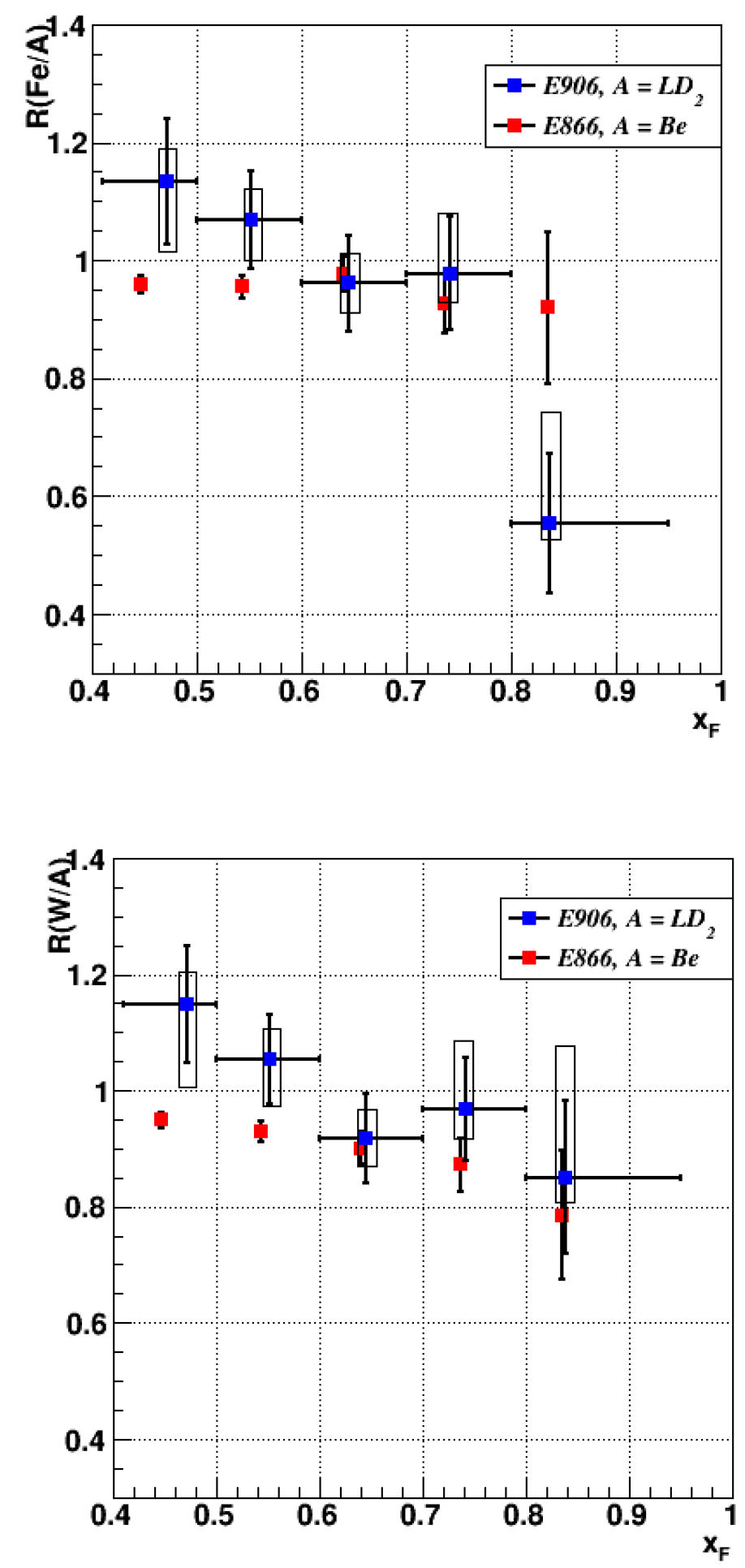

Figure 4.10: DY $R_{p A}$ vs $x_{F}$ for iron and tungsten as measured in E906 and E866. Liquid deuterium $\left(L D_{2}\right)$ was the base target for E906 ratio measurements, while Beryllium $(\mathrm{Be})$ was the base target for E866 ratio measurements. Boxes around points represent systematic error for E906. E866 reported a systematic error of $1 \%$ across all $x_{F}$ bins [27]. 


\section{Chapter 5 Summary}

With its ability to measure dimuon events produced by the Drell-Yan process and meson decays as a function of transverse momentum and Feynman-x from a diverse assortment of target species, the E906/SeaQuest experiment can address several pressing questions regarding nucleon structure, parton dynamics and elusive production mechanisms. This dissertation examined $p_{T}$ and $x_{F}$ ratio measurements in order to shed light on the phenomenology of partons and partonic bound states in a variety of nuclear media.

\subsection{Nuclear modification}

Using the Intensity-Extrapolation method, the nuclear dependence of the per-nucleon cross section ratio $R_{p A}$ of $\mathrm{C} / L D_{2}, \mathrm{Fe} / L D_{2}$ and $\mathrm{W} / L D_{2}$ as a function of $p_{T}$ and $x_{F}$ was reported for $J / \psi$ and DrellYan processes. The following result observations were made:

- A significant $A$ dependence in which there is a depletion of low- $p_{T}$ dimuons and an excess of high- $p_{T}$ dimuons relative to deuterium was noted for DY.

- For $J / \psi p_{T}$ ratios, a striking $A$ dependence was observed, manifesting in a systematically increasing suppression (from carbon to tungsten).

- A small dependence with $p_{T}$ is seen in the $R_{p A}$ of tungsten but one is not discernible in lighter solid targets for $J / \psi p_{T}$ ratios.

- For DY $x_{F}$ ratios, a small $A$ dependence for the drop in $R_{p A}$ for increasing $x_{F}$ is also noted. An analysis cut $x_{T}>0.16$ (which removes the effects of shadowing) reports consistent 
results without the cut indicating that the effects of shadowing are small.

- For $J / \psi x_{F}$ ratios, a similar $x_{F}$ dependence is observed but with a substantially more pronounced $A$ dependence characterized by an increasing ratio suppression in heavier targets.

The most notable observation from the comparison plots to E866 is that all trends with $p_{T}$ and $x_{F}$ that were observed in the E866 data are confirmed by these results (especially so for DY). There is a slight change in the $J / \psi x_{F}$ trend for both E906 and E866 at $x_{F} \quad 0.75$. Individually, this could simply be attributed to statistical fluctuations but given that it is observed in both experiments it is something worth examining further. $J / \psi$ ratios as a function of both variables are consistently below the E866 ratios, which could be in part due to the differences in target bases $\left(L D_{2} \mathrm{vs}\right.$. Be). However, it is also likely due to a combination of other effects such as the greater contribution from $q \bar{q}$ in the data and thus a contrasting mix of nPDFs, a different initial-state energy loss of the lower-momentum beam parton in the case of SeaQuest, and even differences in the $J / \psi$ formation (color-neutralization) from the $c \bar{c}$ pair. Lastly, final-state interactions for the $J / \psi$ could have a non-trivial dependence on the energy of the incident proton beam, with a $120 \mathrm{GeV}$ beam for E906 vs. an $800 \mathrm{GeV}$ beam for E866. The lower the energy of the projectile proton, the more prominent the effect of the nuclear medium could be on its production.

These results, in conjunction with others, will contribute to our collective understanding of quark and anti-quark distribution modifications in nuclei and could serve as important constraints for better modeling of parton energy loss in cold nuclear media. In particular, the results for $J / \psi$ production modification and its varying dependences will shed light on the different initial and final-state mechanisms guiding and influencing $J / \psi$ meson generation. Lastly, they will contribute to a better quantitative understanding of $\mathrm{CNM}$ effects and could serve as baseline measurements for the purpose of better characterizing signatures of QGP screening. 


\subsection{Future objectives}

\subsubsection{More data!}

Approximately $30 \%$ of the data recorded at SeaQuest is used in this dissertation. Later runs include the presence of the new chamber, DC1.2, which should yield a wider acceptance and increase the amount of data available in the high- $x$ region. One can use this data to do a crosscheck of the results shown in this dissertation. Moreover, one can revisit many phenomenological questions that can only be accessed and addressed with more high- $x$ data. After appropriate calibrations and the development of a revised track reconstruction software, efforts to include this additional data in this analysis will be set into motion.

\subsubsection{Systematic uncertainties improvement}

The sources of systematic uncertainties that were considered were discussed in Section 4.2. A major source of systematic uncertainty for both processes comes from the choice of the fitting function. The uncertainties cannot identify a unique fitting function at present. However, with the inclusion of more data, the fits will be more constrained and the systematic uncertainties coming from the choice of the fitting function could be reduced. In addition, the $F$ contamination factor could be estimated on a bin-by-bin basis (both for $p_{T}$ and $x_{F}$ ) using projections of the mass fit to these variables. Studies to improve MC for all physics processes for $p_{T}$ are underway and should make a bin-by-bin treatment of this factor more feasible.

\subsubsection{Nuclear dependence for different target bases: $\mathrm{C}$ and $\mathrm{LH}_{2}$}

The IE method can also be utilized to examine the nuclear dependence of several quantities in the per-nucleon ratio of cross-sections for $\mathrm{Fe} / \mathrm{C}$ and $\mathrm{W} / \mathrm{C}$, as well as $\mathrm{C} / \mathrm{LH}_{2}, \mathrm{Fe} / \mathrm{LH}_{2}$ and $\mathrm{W} / \mathrm{LH}_{2}$ rather than $\mathrm{Fe} / L D_{2}$ and $W / L D_{2}$. With carbon being more similar in size to beryllium than deuterium, these measurements could make for more direct comparisons with the E866 results. In addition, with $\mathrm{LH}_{2}$ being lighter target than $\mathrm{LD}_{2}$ could possible help constrain nuclear effects for 
$L D_{2}$. These complementary research efforts are already underway. 
Appendices 


\section{Appendix A \\ Raw yields and intensity dependence plots}

\section{A.1 Raw dimuon yield tables}

\section{A.1.1 $p_{T}$ yields for $J / \psi$}

\begin{tabular}{|c|c|c|c|c|c|c|c|c|}
\hline \multicolumn{10}{|c|}{$\left(0.0<p_{T}<0.33\right)$} \\
\hline Intensity min & Intensity max & $\mathrm{LH}_{2}$ & EMPTY & $\mathrm{LD}_{2}$ & None & $\mathrm{Fe}$ & $\mathrm{C}$ & $\mathrm{W}$ \\
\hline 0 & 64000 & 11132 & 273 & 11370 & 202 & 3060 & 3503 & 2803 \\
\hline 0 & 8000 & 1778 & 42 & 1907 & 25 & 500 & 590 & 400 \\
\hline 8000 & 16000 & 2356 & 45 & 2512 & 32 & 631 & 702 & 637 \\
\hline 16000 & 24000 & 2120 & 41 & 2187 & 36 & 611 & 654 & 570 \\
\hline 24000 & 32000 & 1764 & 51 & 1726 & 32 & 464 & 554 & 389 \\
\hline 32000 & 40000 & 1254 & 39 & 1207 & 29 & 335 & 406 & 329 \\
\hline 40000 & 48000 & 839 & 24 & 833 & 21 & 216 & 272 & 213 \\
\hline 48000 & 56000 & 618 & 17 & 570 & 18 & 172 & 200 & 158 \\
\hline 56000 & 64000 & 403 & 14 & 428 & 9 & 131 & 125 & 107 \\
\hline
\end{tabular}

Table A.1: Dimuon yields per intensity bin for $0.0<p_{T}<0.33$.

\begin{tabular}{|c|c|c|c|c|c|c|c|c|}
\hline \multicolumn{10}{|c|}{$\left(0.33<p_{T}<0.54\right)$} \\
\hline Intensity min & Intensity max & $\mathrm{LH}_{2}$ & EMPTY & $\mathrm{LD}_{2}$ & None & $\mathrm{Fe}$ & $\mathrm{C}$ & $\mathrm{W}$ \\
\hline 0 & 64000 & 12285 & 227 & 12488 & 199 & 3387 & 3948 & 3231 \\
\hline 0 & 8000 & 1849 & 25 & 2128 & 20 & 558 & 623 & 494 \\
\hline 8000 & 16000 & 2511 & 33 & 2750 & 42 & 684 & 862 & 671 \\
\hline 16000 & 24000 & 2393 & 44 & 2432 & 27 & 642 & 746 & 594 \\
\hline 24000 & 32000 & 1978 & 38 & 1874 & 39 & 578 & 577 & 522 \\
\hline 32000 & 40000 & 1448 & 33 & 1322 & 27 & 353 & 446 & 369 \\
\hline 40000 & 48000 & 990 & 30 & 952 & 22 & 265 & 322 & 276 \\
\hline 48000 & 56000 & 662 & 15 & 602 & 14 & 165 & 207 & 180 \\
\hline 56000 & 64000 & 454 & 9 & 428 & 8 & 142 & 165 & 125 \\
\hline
\end{tabular}

Table A.2: Dimuon yields per intensity bin for $0.33<p_{T}<0.54$. 


\begin{tabular}{|c|c|c|c|c|c|c|c|c|}
\hline \multicolumn{8}{|c|}{$\left(0.54<p_{T}<0.72\right)$} \\
\hline Intensity min & Intensity max & $\mathrm{LH}_{2}$ & EMPTY & $\mathrm{LD}_{2}$ & None & Fe & $\mathrm{C}$ & $\mathrm{W}$ \\
\hline 0 & 64000 & 8299 & 179 & 8430 & 130 & 2353 & 2712 & 2326 \\
\hline 0 & 8000 & 1206 & 23 & 1307 & 8 & 352 & 393 & 325 \\
\hline 8000 & 16000 & 1764 & 33 & 1832 & 20 & 465 & 557 & 462 \\
\hline 16000 & 24000 & 1558 & 33 & 1628 & 33 & 473 & 534 & 463 \\
\hline 24000 & 32000 & 1273 & 29 & 1359 & 29 & 364 & 418 & 376 \\
\hline 32000 & 40000 & 975 & 26 & 944 & 15 & 278 & 305 & 260 \\
\hline 40000 & 48000 & 718 & 15 & 589 & 11 & 182 & 245 & 198 \\
\hline 48000 & 56000 & 485 & 10 & 443 & 11 & 140 & 159 & 134 \\
\hline 56000 & 64000 & 320 & 10 & 328 & 3 & 99 & 101 & 108 \\
\hline
\end{tabular}

Table A.3: Dimuon yields per intensity bin for $0.54<p_{T}<0.72$.

\begin{tabular}{|c|c|c|c|c|c|c|c|c|c|}
\hline \multicolumn{10}{|c|}{$\left(0.72<p_{T}<0.99\right)$} \\
\hline Intensity min & Intensity max & $\mathrm{LH}_{2}$ & EMPTY & $\mathrm{LD}_{2}$ & None & Fe & $\mathrm{C}$ & $\mathrm{W}$ \\
\hline 0 & 64000 & 7223 & 150 & 7278 & 126 & 2029 & 2332 & 2091 \\
\hline 0 & 8000 & 994 & 15 & 1131 & 8 & 256 & 359 & 272 \\
\hline 8000 & 16000 & 1508 & 28 & 1558 & 21 & 424 & 510 & 433 \\
\hline 16000 & 24000 & 1400 & 22 & 1389 & 30 & 383 & 435 & 382 \\
\hline 24000 & 32000 & 1156 & 30 & 1151 & 25 & 343 & 365 & 319 \\
\hline 32000 & 40000 & 821 & 22 & 791 & 13 & 240 & 244 & 248 \\
\hline 40000 & 48000 & 610 & 20 & 581 & 11 & 180 & 188 & 198 \\
\hline 48000 & 56000 & 458 & 8 & 373 & 13 & 116 & 136 & 145 \\
\hline 56000 & 64000 & 276 & 5 & 304 & 5 & 87 & 95 & 94 \\
\hline
\end{tabular}

Table A.4: Dimuon yields per intensity bin for $0.72<p_{T}<0.99$. 


\begin{tabular}{|c|c|c|c|c|c|c|c|c|}
\hline \multicolumn{10}{|c|}{$\left(0.99<p_{T}<2.3\right)$} \\
\hline Intensity min & Intensity max & $\mathrm{LH}_{2}$ & EMPTY & $\mathrm{LD}_{2}$ & None & Fe & $\mathrm{C}$ & $\mathrm{W}$ \\
\hline 0 & 64000 & 4047 & 99 & 4321 & 82 & 1320 & 1389 & 1332 \\
\hline 0 & 8000 & 556 & 12 & 643 & 9 & 156 & 193 & 168 \\
\hline 8000 & 16000 & 791 & 13 & 891 & 13 & 255 & 262 & 240 \\
\hline 16000 & 24000 & 747 & 20 & 841 & 13 & 270 & 246 & 262 \\
\hline 24000 & 32000 & 673 & 14 & 654 & 12 & 214 & 226 & 213 \\
\hline 32000 & 40000 & 510 & 11 & 489 & 12 & 138 & 168 & 165 \\
\hline 40000 & 48000 & 328 & 16 & 374 & 10 & 117 & 125 & 119 \\
\hline 48000 & 56000 & 261 & 7 & 248 & 5 & 93 & 91 & 92 \\
\hline 56000 & 64000 & 181 & 6 & 181 & 8 & 77 & 78 & 73 \\
\hline
\end{tabular}

Table A.5: Dimuon yields per intensity bin for $0.99<p_{T}<2.3$.

\section{A.1.2 $x_{F}$ yields for $J / \psi$}

\begin{tabular}{|c|c|c|c|c|c|c|c|c|}
\hline \multicolumn{9}{|c|}{$\left(0.0<x_{F}<0.5\right)$} \\
\hline Intensity min & Intensity max & $\mathrm{LH}_{2}$ & EMPTY & $\mathrm{LD}_{2}$ & None & $\mathrm{C}$ & $\mathrm{Fe}$ & $\mathrm{W}$ \\
\hline 0 & 64000 & 4587 & 136 & 4615 & 96 & 1628 & 1417 & 1434 \\
\hline 0 & 8000 & 784 & 20 & 784 & 9 & 292 & 227 & 210 \\
\hline 8000 & 16000 & 968 & 28 & 1063 & 14 & 321 & 286 & 302 \\
\hline 16000 & 24000 & 901 & 23 & 935 & 22 & 320 & 290 & 273 \\
\hline 24000 & 32000 & 704 & 20 & 683 & 21 & 237 & 240 & 228 \\
\hline 32000 & 40000 & 488 & 20 & 498 & 10 & 176 & 146 & 166 \\
\hline 40000 & 48000 & 344 & 12 & 296 & 8 & 125 & 87 & 116 \\
\hline 48000 & 56000 & 229 & 6 & 220 & 9 & 87 & 84 & 77 \\
\hline 56000 & 64000 & 169 & 7 & 136 & 3 & 70 & 57 & 62 \\
\hline
\end{tabular}

Table A.6: Dimuon yields per intensity bin for $0.0<x_{F}<0.5$. 


\begin{tabular}{|c|c|c|c|c|c|c|c|c|}
\hline \multicolumn{8}{|c|}{$\left(0.5<x_{F}<0.6\right)$} \\
\hline Intensity min & Intensity max & $\mathrm{LH}_{2}$ & EMPTY & $\mathrm{LD}_{2}$ & None & $\mathrm{C}$ & Fe & $\mathrm{W}$ \\
\hline 0 & 64000 & 15386 & 335 & 15304 & 257 & 4899 & 4409 & 4329 \\
\hline 0 & 8000 & 2347 & 29 & 2617 & 26 & 774 & 682 & 630 \\
\hline 8000 & 16000 & 3265 & 60 & 3430 & 45 & 1076 & 918 & 940 \\
\hline 16000 & 24000 & 2964 & 55 & 2990 & 48 & 913 & 874 & 875 \\
\hline 24000 & 32000 & 2483 & 63 & 2270 & 52 & 760 & 730 & 669 \\
\hline 32000 & 40000 & 1773 & 57 & 1614 & 29 & 559 & 464 & 483 \\
\hline 40000 & 48000 & 1206 & 35 & 1120 & 24 & 375 & 315 & 343 \\
\hline 48000 & 56000 & 828 & 19 & 709 & 20 & 274 & 222 & 222 \\
\hline 56000 & 64000 & 520 & 17 & 554 & 10 & 168 & 204 & 167 \\
\hline
\end{tabular}

Table A.7: Dimuon yields per intensity bin for $0.5<x_{F}<0.6$.

\begin{tabular}{|c|c|c|c|c|c|c|c|c|}
\hline \multicolumn{8}{|c|}{$\left(0.6<x_{F}<0.7\right)$} \\
\hline Intensity min & Intensity max & $\mathrm{LH}_{2}$ & EMPTY & $\mathrm{LD}_{2}$ & None & $\mathrm{C}$ & Fe & W \\
\hline 0 & 64000 & 15329 & 313 & 15880 & 256 & 4923 & 4211 & 3997 \\
\hline 0 & 8000 & 2215 & 50 & 2544 & 28 & 784 & 620 & 583 \\
\hline 8000 & 16000 & 3200 & 42 & 3426 & 46 & 1024 & 891 & 815 \\
\hline 16000 & 24000 & 2940 & 60 & 3023 & 45 & 932 & 811 & 753 \\
\hline 24000 & 32000 & 2434 & 50 & 2450 & 39 & 734 & 658 & 599 \\
\hline 32000 & 40000 & 1760 & 40 & 1757 & 41 & 544 & 467 & 468 \\
\hline 40000 & 48000 & 1252 & 34 & 1200 & 30 & 421 & 371 & 353 \\
\hline 48000 & 56000 & 925 & 24 & 841 & 17 & 274 & 239 & 259 \\
\hline 56000 & 64000 & 603 & 13 & 639 & 10 & 210 & 154 & 167 \\
\hline
\end{tabular}

Table A.8: Dimuon yields per intensity bin for $0.6<x_{F}<0.7$. 


\begin{tabular}{|c|c|c|c|c|c|c|c|c|}
\hline \multicolumn{9}{|c|}{$\left(0.7<x_{F}<0.8\right)$} \\
\hline Intensity min & Intensity max & $\mathrm{LH}_{2}$ & EMPTY & $\mathrm{LD}_{2}$ & None & $\mathrm{C}$ & Fe & $\mathrm{W}$ \\
\hline 0 & 64000 & 6474 & 119 & 6720 & 104 & 2034 & 1771 & 1744 \\
\hline 0 & 8000 & 892 & 16 & 1009 & 6 & 254 & 256 & 203 \\
\hline 8000 & 16000 & 1269 & 18 & 1364 & 18 & 400 & 309 & 339 \\
\hline 16000 & 24000 & 1196 & 19 & 1281 & 21 & 375 & 333 & 322 \\
\hline 24000 & 32000 & 1033 & 20 & 1106 & 21 & 345 & 273 & 288 \\
\hline 32000 & 40000 & 842 & 12 & 734 & 12 & 239 & 218 & 208 \\
\hline 40000 & 48000 & 564 & 20 & 585 & 10 & 191 & 167 & 163 \\
\hline 48000 & 56000 & 406 & 7 & 376 & 11 & 129 & 116 & 130 \\
\hline 56000 & 64000 & 272 & 7 & 265 & 5 & 101 & 99 & 91 \\
\hline
\end{tabular}

Table A.9: Dimuon yields per intensity bin for $0.7<x_{F}<0.8$.

\begin{tabular}{|c|c|c|c|c|c|c|c|c|}
\hline \multicolumn{10}{|c|}{$\left(0.8<x_{F}<0.95\right)$} \\
\hline Intensity min & Intensity max & $\mathrm{LH}_{2}$ & EMPTY & $\mathrm{LD}_{2}$ & None & $\mathrm{C}$ & Fe & W \\
\hline 0 & 64000 & 1209 & 25 & 1368 & 26 & 400 & 341 & 279 \\
\hline 0 & 8000 & 145 & 2 & 162 & 1 & 54 & 37 & 33 \\
\hline 8000 & 16000 & 227 & 4 & 260 & 5 & 72 & 55 & 47 \\
\hline 16000 & 24000 & 217 & 3 & 248 & 3 & 75 & 71 & 48 \\
\hline 24000 & 32000 & 190 & 9 & 255 & 4 & 64 & 62 & 35 \\
\hline 32000 & 40000 & 145 & 2 & 150 & 4 & 51 & 49 & 46 \\
\hline 40000 & 48000 & 119 & 4 & 128 & 3 & 40 & 20 & 29 \\
\hline 48000 & 56000 & 96 & 1 & 90 & 4 & 29 & 25 & 21 \\
\hline 56000 & 64000 & 70 & 0 & 75 & 2 & 15 & 22 & 20 \\
\hline
\end{tabular}

Table A.10: Dimuon yields per intensity bin for $0.8<x_{F}<0.95$. 


\section{A.1.3 $p_{T}$ Yields for DY}

\begin{tabular}{|c|c|c|c|c|c|c|c|c|}
\hline \multicolumn{10}{|c|}{$\left(0.0<p_{T}<0.33\right)$} \\
\hline Intensity min & Intensity max & $\mathrm{LH}_{2}$ & EMPTY & $\mathrm{LD}_{2}$ & None & $\mathrm{Fe}$ & $\mathrm{C}$ & $\mathrm{W}$ \\
\hline 0 & 64000 & 1043 & 32 & 1257 & 16 & 436 & 423 & 542 \\
\hline 0 & 8000 & 176 & 8 & 189 & 2 & 72 & 62 & 74 \\
\hline 8000 & 16000 & 216 & 2 & 271 & 3 & 99 & 92 & 123 \\
\hline 16000 & 24000 & 189 & 11 & 251 & 2 & 78 & 74 & 112 \\
\hline 24000 & 32000 & 162 & 3 & 192 & 3 & 69 & 73 & 68 \\
\hline 32000 & 40000 & 112 & 5 & 139 & 3 & 37 & 54 & 79 \\
\hline 40000 & 48000 & 98 & 1 & 100 & 1 & 54 & 32 & 40 \\
\hline 48000 & 56000 & 59 & 2 & 74 & 1 & 12 & 17 & 27 \\
\hline 56000 & 64000 & 31 & 0 & 41 & 1 & 15 & 19 & 19 \\
\hline
\end{tabular}

Table A.11: Dimuon yields per intensity bin for $0.0<p_{T}<0.33$.

\begin{tabular}{|c|c|c|c|c|c|c|c|c|}
\hline \multicolumn{10}{|c|}{$\left(0.33<p_{T}<0.54\right)$} \\
\hline Intensity min & Intensity max & $\mathrm{LH}_{2}$ & EMPTY & $\mathrm{LD}_{2}$ & None & $\mathrm{Fe}$ & $\mathrm{C}$ & $\mathrm{W}$ \\
\hline 0 & 64000 & 1356 & 34 & 1558 & 26 & 586 & 621 & 675 \\
\hline 0 & 8000 & 203 & 7 & 242 & 3 & 90 & 99 & 104 \\
\hline 8000 & 16000 & 282 & 8 & 327 & 6 & 133 & 125 & 135 \\
\hline 16000 & 24000 & 280 & 6 & 310 & 4 & 110 & 115 & 129 \\
\hline 24000 & 32000 & 198 & 4 & 236 & 3 & 93 & 95 & 118 \\
\hline 32000 & 40000 & 142 & 3 & 189 & 5 & 55 & 78 & 74 \\
\hline 40000 & 48000 & 128 & 3 & 125 & 2 & 50 & 57 & 61 \\
\hline 48000 & 56000 & 69 & 3 & 78 & 2 & 35 & 31 & 40 \\
\hline 56000 & 64000 & 54 & 0 & 51 & 1 & 20 & 21 & 14 \\
\hline
\end{tabular}

Table A.12: Dimuon yields per intensity bin for $0.33<p_{T}<0.54$. 


\begin{tabular}{|c|c|c|c|c|c|c|c|c|}
\hline \multicolumn{10}{|c|}{$\left(0.54<p_{T}<0.72\right)$} \\
\hline Intensity min & Intensity max & $\mathrm{LH}_{2}$ & EMPTY & $\mathrm{LD}_{2}$ & None & Fe & $\mathrm{C}$ & $\mathrm{W}$ \\
\hline 0 & 64000 & 1130 & 44 & 1276 & 22 & 495 & 479 & 613 \\
\hline 0 & 8000 & 174 & 3 & 180 & 3 & 64 & 80 & 91 \\
\hline 8000 & 16000 & 259 & 15 & 282 & 5 & 95 & 98 & 131 \\
\hline 16000 & 24000 & 225 & 4 & 252 & 4 & 97 & 88 & 127 \\
\hline 24000 & 32000 & 156 & 8 & 215 & 3 & 88 & 75 & 111 \\
\hline 32000 & 40000 & 126 & 5 & 134 & 4 & 71 & 55 & 62 \\
\hline 40000 & 48000 & 95 & 6 & 103 & 0 & 35 & 43 & 40 \\
\hline 48000 & 56000 & 50 & 2 & 61 & 0 & 30 & 21 & 29 \\
\hline 56000 & 64000 & 45 & 1 & 49 & 3 & 15 & 19 & 22 \\
\hline
\end{tabular}

Table A.13: Dimuon yields per intensity bin for $0.54<p_{T}<0.72$.

\begin{tabular}{|c|c|c|c|c|c|c|c|c|}
\hline \multicolumn{10}{|c|}{$\left(0.72<p_{T}<0.99\right)$} \\
\hline Intensity min & Intensity max & $\mathrm{LH}_{2}$ & EMPTY & $\mathrm{LD}_{2}$ & None & Fe & $\mathrm{C}$ & $\mathrm{W}$ \\
\hline 0 & 64000 & 1205 & 27 & 1360 & 21 & 612 & 493 & 761 \\
\hline 0 & 8000 & 182 & 4 & 224 & 5 & 85 & 63 & 121 \\
\hline 8000 & 16000 & 258 & 8 & 287 & 5 & 144 & 107 & 152 \\
\hline 16000 & 24000 & 218 & 2 & 261 & 3 & 104 & 103 & 126 \\
\hline 24000 & 32000 & 193 & 3 & 216 & 3 & 84 & 85 & 119 \\
\hline 32000 & 40000 & 120 & 3 & 149 & 4 & 80 & 40 & 97 \\
\hline 40000 & 48000 & 107 & 4 & 88 & 1 & 51 & 42 & 71 \\
\hline 48000 & 56000 & 67 & 3 & 83 & 0 & 34 & 31 & 50 \\
\hline 56000 & 64000 & 60 & 0 & 52 & 0 & 30 & 22 & 25 \\
\hline
\end{tabular}

Table A.14: Dimuon yields per intensity bin for $0.72<p_{T}<0.99$. 


\begin{tabular}{|c|c|c|c|c|c|c|c|c|c|}
\hline \multicolumn{10}{|c|}{$\left(0.99<p_{T}<2.3\right)$} \\
\hline Intensity min & Intensity max & $\mathrm{LH}_{2}$ & EMPTY & $\mathrm{LD}_{2}$ & None & Fe & $\mathrm{C}$ & W \\
\hline 0 & 64000 & 895 & 27 & 1073 & 21 & 494 & 383 & 618 \\
\hline 0 & 8000 & 107 & 2 & 145 & 2 & 71 & 41 & 91 \\
\hline 8000 & 16000 & 167 & 7 & 196 & 0 & 115 & 80 & 110 \\
\hline 16000 & 24000 & 160 & 8 & 235 & 5 & 78 & 82 & 116 \\
\hline 24000 & 32000 & 141 & 5 & 177 & 8 & 76 & 64 & 89 \\
\hline 32000 & 40000 & 112 & 1 & 125 & 2 & 64 & 51 & 76 \\
\hline 40000 & 48000 & 91 & 3 & 84 & 2 & 37 & 28 & 62 \\
\hline 48000 & 56000 & 63 & 0 & 65 & 0 & 30 & 18 & 45 \\
\hline 56000 & 64000 & 54 & 1 & 46 & 2 & 23 & 19 & 29 \\
\hline
\end{tabular}

Table A.15: Dimuon yields per intensity bin for $0.99<p_{T}<2.3$.

\section{A.1.4 $x_{F}$ Yields for DY}

\begin{tabular}{|c|c|c|c|c|c|c|c|c|}
\hline \multicolumn{10}{|c|}{$\left(0.0<x_{F}<0.5\right)$} \\
\hline Intensity min & Intensity max & $\mathrm{LH}_{2}$ & EMPTY & $\mathrm{LD}_{2}$ & None & $\mathrm{C}$ & $\mathrm{Fe}$ & $\mathrm{W}$ \\
\hline 0 & 64000 & 704 & 30 & 780 & 13 & 342 & 272 & 423 \\
\hline 0 & 8000 & 106 & 2 & 113 & 2 & 50 & 45 & 60 \\
\hline 8000 & 16000 & 165 & 6 & 165 & 2 & 66 & 51 & 87 \\
\hline 16000 & 24000 & 130 & 10 & 158 & 1 & 72 & 56 & 85 \\
\hline 24000 & 32000 & 94 & 6 & 124 & 3 & 56 & 50 & 74 \\
\hline 32000 & 40000 & 80 & 1 & 73 & 2 & 42 & 26 & 45 \\
\hline 40000 & 48000 & 60 & 2 & 64 & 1 & 29 & 23 & 37 \\
\hline 48000 & 56000 & 43 & 3 & 59 & 0 & 15 & 9 & 21 \\
\hline 56000 & 64000 & 26 & 0 & 24 & 2 & 12 & 12 & 14 \\
\hline
\end{tabular}

Table A.16: Dimuon yields per intensity bin for $0.0<x_{F}<0.5$. 


\begin{tabular}{|c|c|c|c|c|c|c|c|c|}
\hline \multicolumn{10}{|c|}{$\left(0.5<x_{F}<0.6\right)$} \\
\hline Intensity min & Intensity max & $\mathrm{LH}_{2}$ & EMPTY & $\mathrm{LD}_{2}$ & None & $\mathrm{C}$ & Fe & W \\
\hline 0 & 64000 & 2066 & 56 & 2334 & 45 & 993 & 836 & 1215 \\
\hline 0 & 8000 & 305 & 9 & 348 & 4 & 136 & 109 & 181 \\
\hline 8000 & 16000 & 418 & 15 & 499 & 8 & 233 & 195 & 250 \\
\hline 16000 & 24000 & 405 & 8 & 480 & 8 & 170 & 162 & 216 \\
\hline 24000 & 32000 & 317 & 5 & 373 & 8 & 161 & 126 & 202 \\
\hline 32000 & 40000 & 218 & 4 & 261 & 9 & 118 & 96 & 136 \\
\hline 40000 & 48000 & 204 & 9 & 164 & 4 & 83 & 66 & 110 \\
\hline 48000 & 56000 & 112 & 5 & 120 & 2 & 55 & 49 & 77 \\
\hline 56000 & 64000 & 87 & 1 & 89 & 2 & 37 & 33 & 43 \\
\hline
\end{tabular}

Table A.17: Dimuon yields per intensity bin for $0.5<x_{F}<0.6$.

\begin{tabular}{|c|c|c|c|c|c|c|c|c|}
\hline \multicolumn{10}{|c|}{$\left(0.6<x_{F}<0.7\right)$} \\
\hline Intensity min & Intensity max & $\mathrm{LH}_{2}$ & EMPTY & $\mathrm{LD}_{2}$ & None & $\mathrm{C}$ & Fe & W \\
\hline 0 & 64000 & 790 & 48 & 2174 & 31 & 824 & 823 & 994 \\
\hline 0 & 8000 & 278 & 7 & 348 & 5 & 121 & 114 & 154 \\
\hline 8000 & 16000 & 386 & 14 & 445 & 7 & 182 & 177 & 197 \\
\hline 16000 & 24000 & 341 & 7 & 412 & 7 & 146 & 150 & 190 \\
\hline 24000 & 32000 & 271 & 9 & 370 & 6 & 134 & 132 & 133 \\
\hline 32000 & 40000 & 190 & 5 & 235 & 4 & 83 & 108 & 140 \\
\hline 40000 & 48000 & 149 & 4 & 164 & 0 & 78 & 63 & 86 \\
\hline 48000 & 56000 & 97 & 1 & 110 & 0 & 45 & 44 & 61 \\
\hline 56000 & 64000 & 78 & 1 & 90 & 2 & 35 & 35 & 33 \\
\hline
\end{tabular}

Table A.18: Dimuon yields per intensity bin for $0.6<x_{F}<0.7$. 


\begin{tabular}{|c|c|c|c|c|c|c|c|c|}
\hline \multicolumn{10}{|c|}{$\left(0.7<x_{F}<0.8\right)$} \\
\hline Intensity min & Intensity max & LH $_{2}$ & EMPTY & LD $_{2}$ & None & $\mathrm{C}$ & Fe & W \\
\hline 0 & 64000 & 877 & 25 & 995 & 13 & 389 & 386 & 469 \\
\hline 0 & 8000 & 127 & 4 & 143 & 3 & 68 & 67 & 74 \\
\hline 8000 & 16000 & 172 & 4 & 208 & 2 & 80 & 63 & 94 \\
\hline 16000 & 24000 & 165 & 6 & 210 & 2 & 69 & 83 & 91 \\
\hline 24000 & 32000 & 140 & 3 & 144 & 3 & 54 & 63 & 86 \\
\hline 32000 & 40000 & 95 & 6 & 127 & 2 & 51 & 37 & 56 \\
\hline 40000 & 48000 & 87 & 2 & 81 & 0 & 32 & 43 & 30 \\
\hline 48000 & 56000 & 46 & 0 & 58 & 0 & 17 & 15 & 26 \\
\hline 56000 & 64000 & 45 & 0 & 24 & 1 & 18 & 15 & 12 \\
\hline
\end{tabular}

Table A.19: Dimuon yields per intensity bin for $0.7<x_{F}<0.8$.

\begin{tabular}{|c|c|c|c|c|c|c|c|c|}
\hline \multicolumn{8}{|c|}{$\left(0.8<x_{F}<0.95\right)$} \\
\hline Intensity min & Intensity max & $\mathrm{LH}_{2}$ & EMPTY & $\mathrm{LD}_{2}$ & None & $\mathrm{C}$ & $\mathrm{Fe}$ & $\mathrm{W}$ \\
\hline 0 & 64000 & 192 & 5 & 241 & 4 & 75 & 82 & 108 \\
\hline 0 & 8000 & 26 & 2 & 28 & 1 & 7 & 10 & 12 \\
\hline 8000 & 16000 & 41 & 1 & 46 & 0 & 25 & 16 & 23 \\
\hline 16000 & 24000 & 31 & 0 & 49 & 0 & 10 & 11 & 28 \\
\hline 24000 & 32000 & 28 & 0 & 25 & 0 & 5 & 21 & 10 \\
\hline 32000 & 40000 & 29 & 1 & 40 & 1 & 13 & 11 & 11 \\
\hline 40000 & 48000 & 19 & 0 & 27 & 1 & 5 & 7 & 11 \\
\hline 48000 & 56000 & 10 & 1 & 14 & 1 & 9 & 1 & 6 \\
\hline 56000 & 64000 & 8 & 0 & 12 & 0 & 1 & 5 & 7 \\
\hline
\end{tabular}

Table A.20: Dimuon yields per intensity bin for $0.8<x_{F}<0.95$.

\section{A.2 $R_{p A}$ intensity dependence plots}

\section{A.2.1 $p_{T}$ plots $J / \psi$}




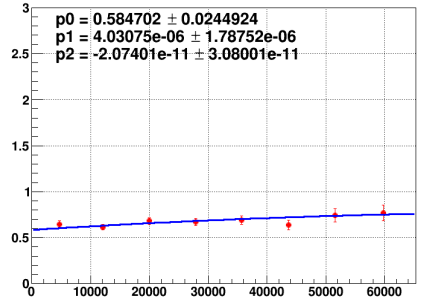

(a) $0.0<p_{T}<0.33$

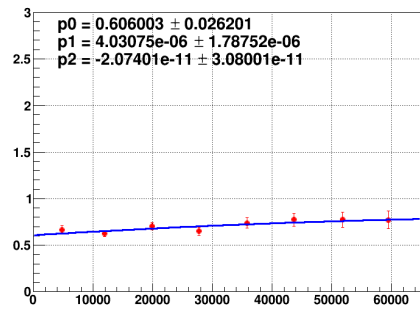

(c) $0.54<p_{T}<0.72$

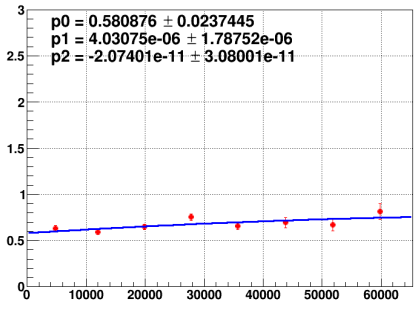

(b) $0.33<p_{T}<0.54$

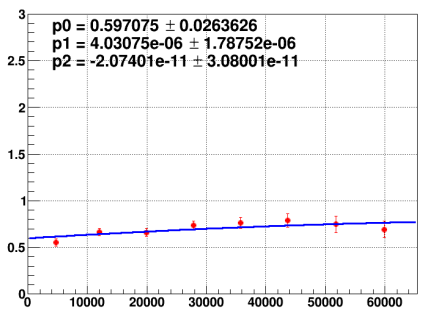

(d) $0.72<p_{T}<0.99$

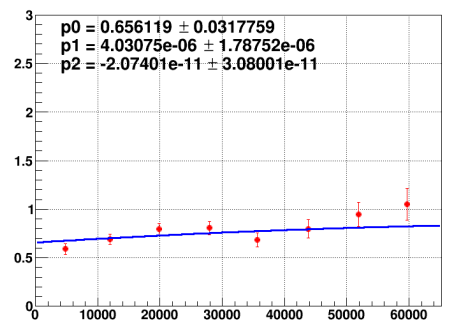

(e) $0.99<p_{T}<2.3$

Figure A.1: Intensity dependence of $J / \psi R_{p A}$ for iron for different $p_{T}(\mathrm{GeV} / \mathrm{c})$ bins. $F=p 0+$ $p 1 *$ intensity $+p 2 *$ intensity ${ }^{2}$ fit was used for the extrapolation, with $p 0$ taken as the nominal $R_{p A}$ value. The fit parameters $p 1$ and $p 2$ are common for all fits. 


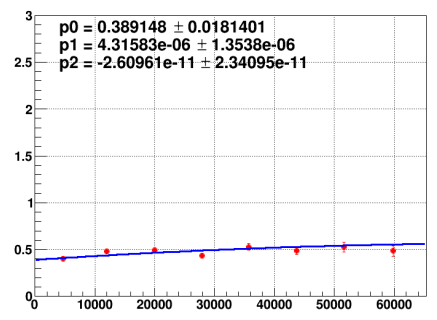

(a) $0.0<p_{T}<0.33$

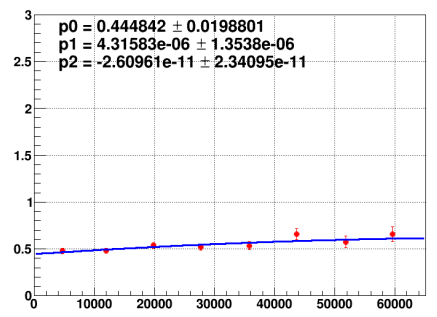

(c) $0.54<p_{T}<0.72$

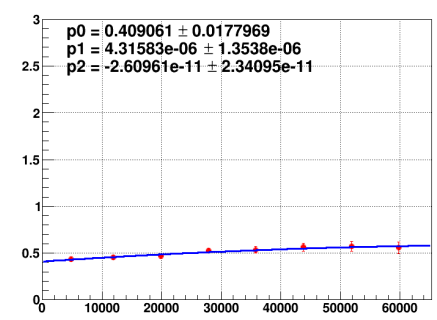

(b) $0.33<p_{T}<0.54$

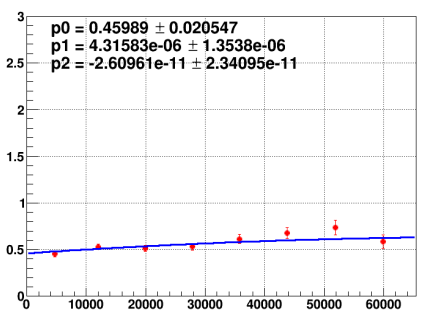

(d) $0.72<p_{T}<0.99$

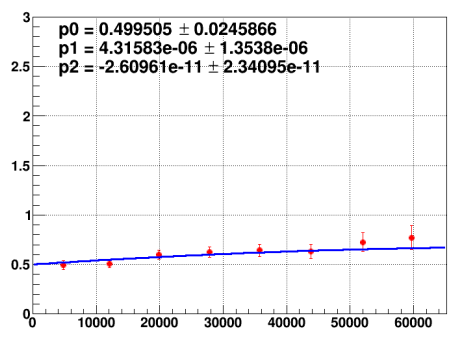

(e) $0.99<p_{T}<2.3$

Figure A.2: Intensity dependnce of $J / \psi R_{p A}$ for tungsten for different $p_{T}(\mathrm{GeV} / \mathrm{c})$ bins. $F=$ $p 0+p 1 *$ intensity $+p 2 *$ intensity ${ }^{2}$ fit was used for the extrapolation, with $p 0$ taken as the nominal $R_{p A}$ value. The fit parameters $p 1$ and $p 2$ are common for all fits. 


\section{A.2.2 $p_{T}$ plots Drell-Yan}

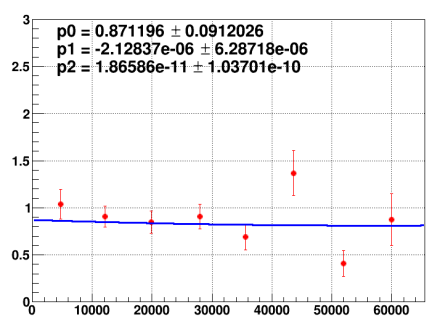

(a) $0.0<p_{T}<0.33$

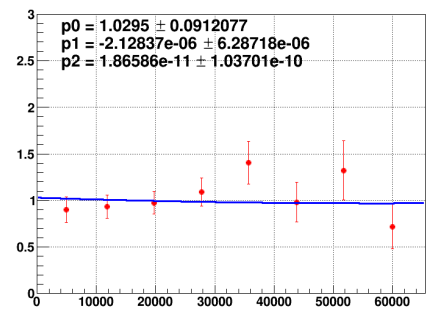

(c) $0.54<p_{T}<0.72$

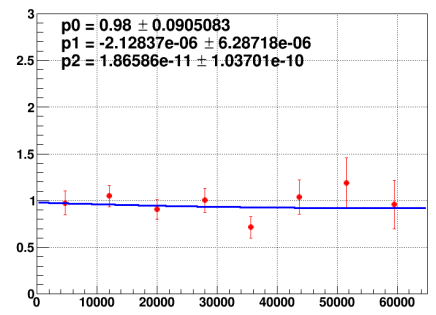

(b) $0.33<p_{T}<0.54$

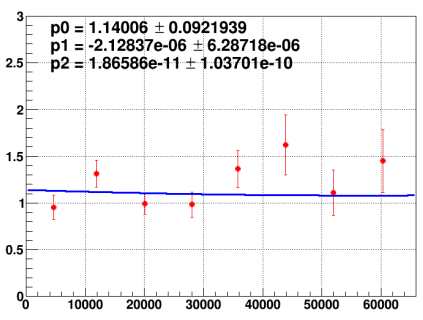

(d) $0.72<p_{T}<0.99$

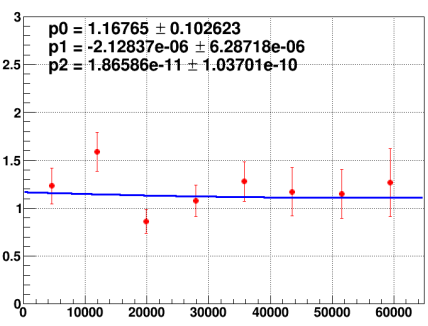

(e) $0.99<p_{T}<2.3$

Figure A.3: Intensity dependence of DY $R_{p A}$ for iron for different $p_{T}(\mathrm{GeV} / \mathrm{c})$ bins. $F=p 0+p 1 *$ intensity $+p 2 *$ intensity ${ }^{2}$ fit was used for the extrapolation, with $p 0$ taken as the nominal $R_{p A}$ value. The fit parameters $p 1$ and $p 2$ are common for all fits. 


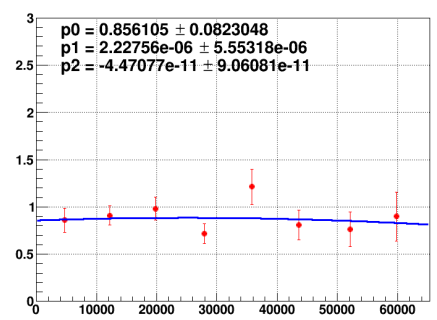

(a) $0.0<p_{T}<0.33$

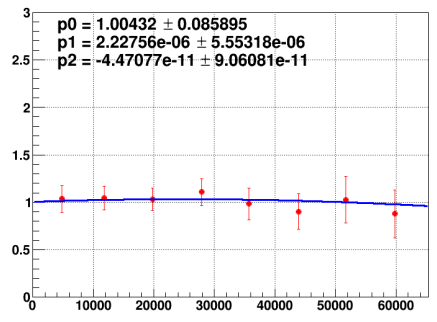

(c) $0.54<p_{T}<0.72$

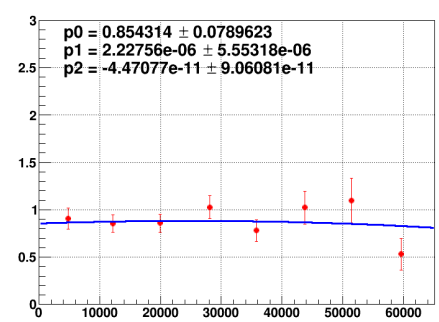

(b) $0.33<p_{T}<0.54$

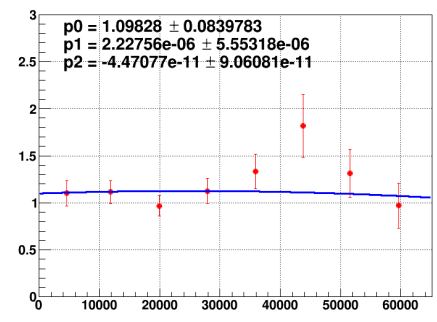

(d) $0.72<p_{T}<0.99$

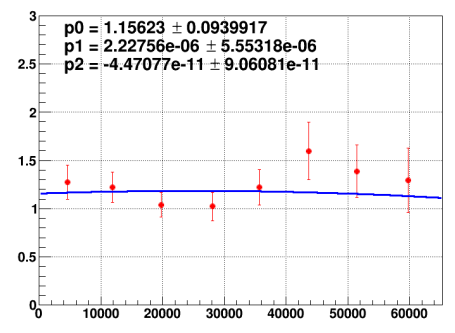

(e) $0.99<p_{T}<2.3$

Figure A.4: Intensity dependence of DY $R_{p A}$ for tungsten for different $p_{T}(\mathrm{GeV} / \mathrm{c})$ bins. $F=$ $p 0+p 1 *$ intensity $+p 2 *$ intensity $^{2}$ fit was used for the extrapolation, with $p 0$ taken as the nominal $R_{p A}$ value. The fit parameters $p 1$ and $p 2$ are common for all fits. 


\section{A.2.3 $x_{F}$ plots $J / \psi$}

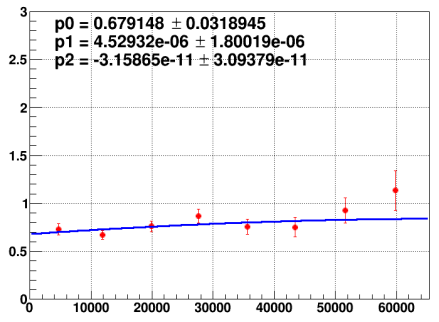

(a) $0.0<x_{F}<0.5$

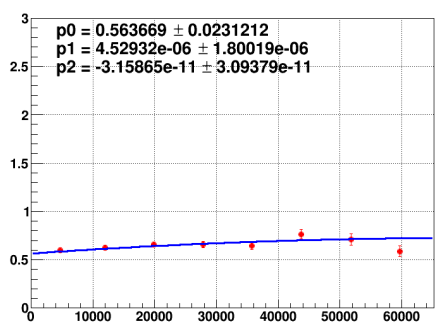

(c) $0.6<x_{F}<0.7$

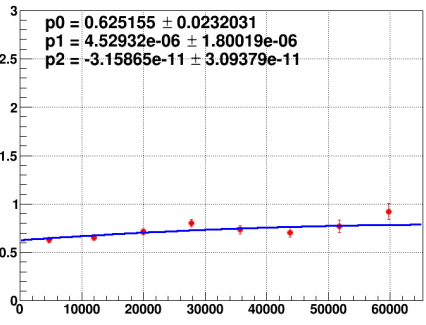

(b) $0.5<x_{F}<0.6$

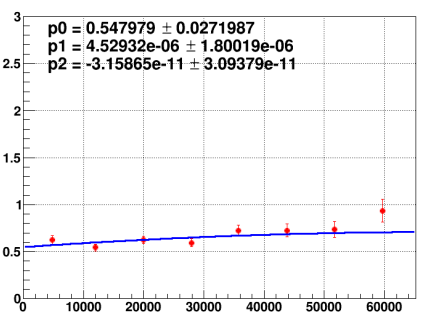

(d) $0.7<x_{F}<0.8$

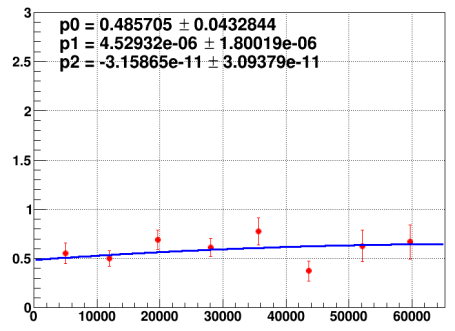

(e) $0.8<x_{F}<0.95$

Figure A.5: Intensity dependence of $J / \psi R_{p A}$ for iron for different $x_{F}$ bins. $F=p 0+p 1 *$ intensity $+p 2 *$ intensity ${ }^{2}$ fit was used for the extrapolation, with $p 0$ taken as the nominal $R_{p A}$ value. The fit parameters $p 1$ and $p 2$ are common for all fits. 


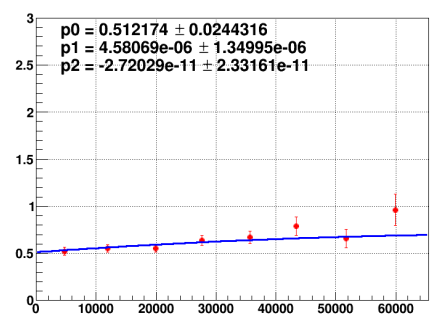

(a) $0.0<x_{F}<0.5$

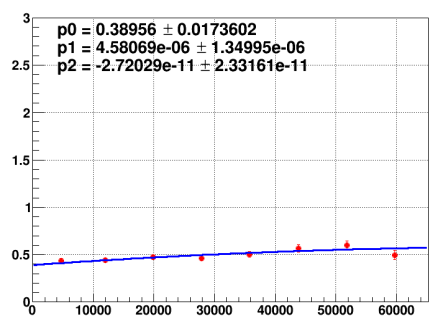

(c) $0.6<x_{F}<0.7$

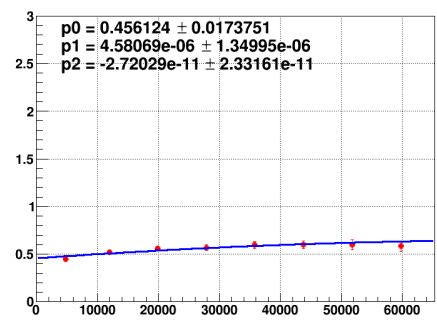

(b) $0.5<x_{F}<0.6$

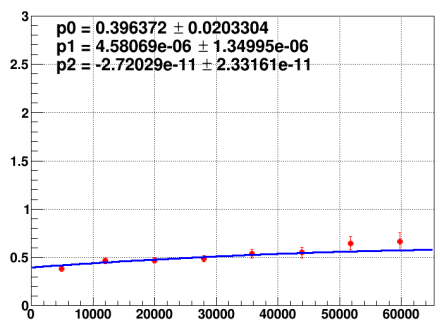

(d) $0.7<x_{F}<0.8$

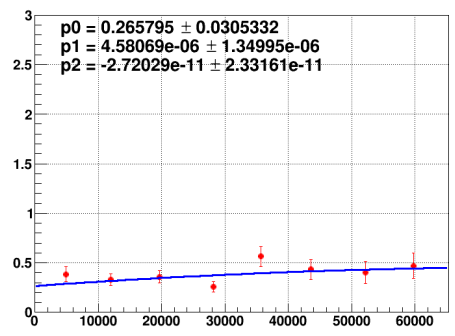

(e) $0.8<x_{F}<0.95$

Figure A.6: Intensity dependence of $J / \psi R_{p A}$ for tungsten for different $x_{F}$ bins. $F=p 0+p 1 *$ intensity $+p 2 *$ intensity ${ }^{2}$ fit was used for the extrapolation, with $p 0$ taken as the nominal $R_{p A}$ value. The fit parameters $p 1$ and $p 2$ are common for all fits. 


\section{A.2.4 $x_{F}$ plots Drell-Yan}

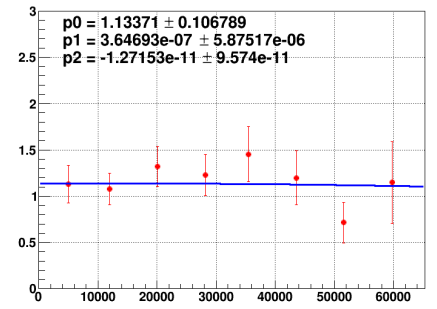

(a) $0.0<x_{F}<0.5$

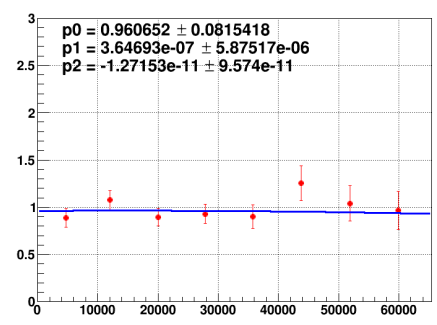

(c) $0.6<x_{F}<0.7$

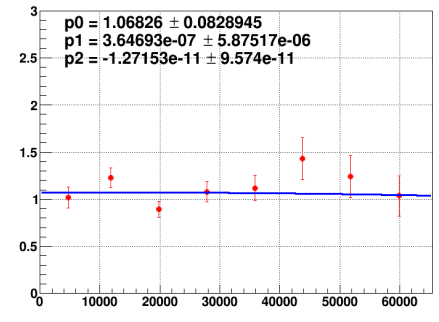

(b) $0.5<x_{F}<0.6$

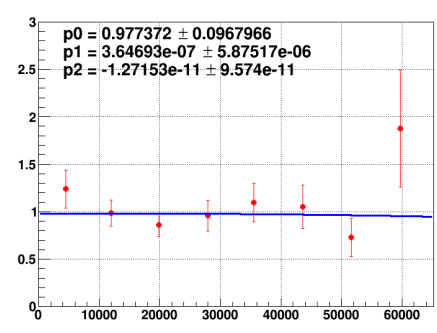

(d) $0.7<x_{F}<0.8$

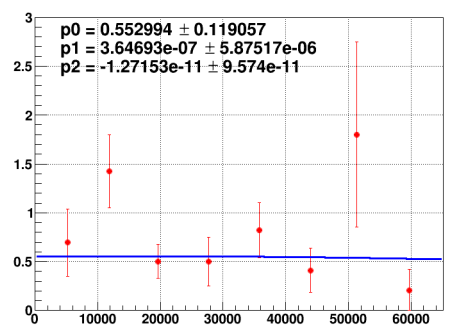

(e) $0.8<x_{F}<0.95$

Figure A.7: Intensity dependence of DY $R_{p A}$ for iron for different $x_{F}$ bins. $F=p 0+p 1 *$ intensity $+p 2 *$ intensity ${ }^{2}$ fit was used for the extrapolation, with $p 0$ taken as the nominal $R_{p A}$ value. The fit parameters $p 1$ and $p 2$ are common for all fits. 


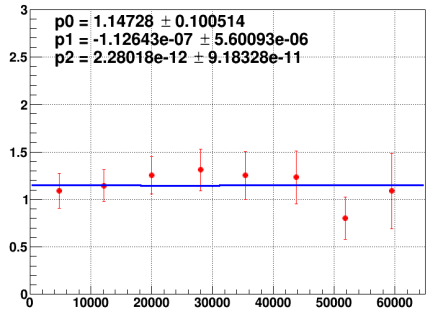

(a) $0.0<x_{F}<0.5$

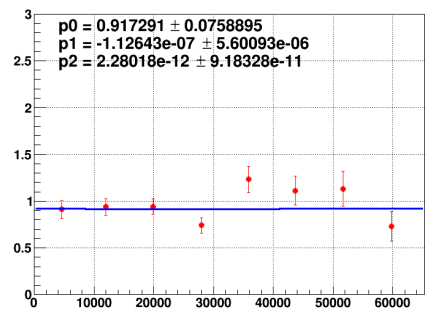

(c) $0.6<x_{F}<0.7$

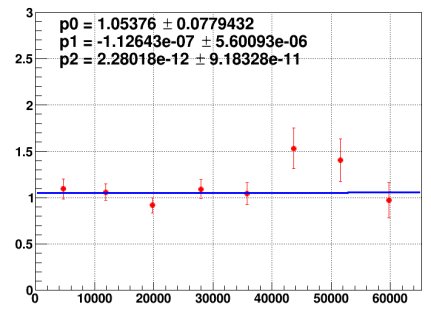

(b) $0.5<x_{F}<0.6$

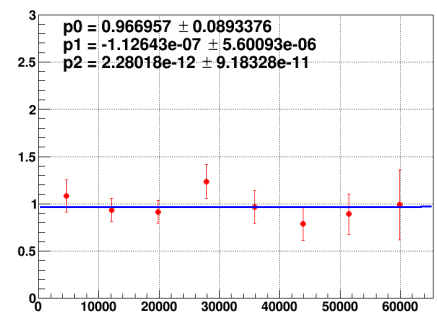

(d) $0.7<x_{F}<0.8$

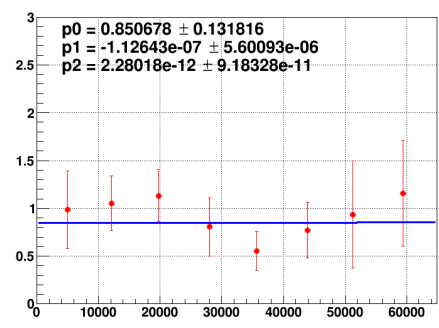

(e) $0.8<x_{F}<0.95$

Figure A.8: Intensity dependence of DY $R_{p A}$ for tungsten for different $x_{F}$ bins. $F=p 0+p 1 *$ intensity $+p 2 *$ intensity ${ }^{2}$ fit was used for the extrapolation, with $p 0$ taken as the nominal $R_{p A}$ value. The fit parameters $p 1$ and $p 2$ are common for all fits. 


\section{References}

[1] CERN, Standard Model, https://home. cern/science/physics/standardmodel, [Online; accessed Jan 22, 2020].

[2] H. Fritzsch, M. Gell-Mann, and H. Leutwyler, "Advantages of the color octet gluon picture," Physics Letters B, vol. 47, no. 4, pp. 365 -368, 1973.

[3] M. Tanabashi et al., "Review of Particle Physics," Phys. Rev. D, vol. 98, p. 030 001, 32018.

[4] A. S. TADEPALLI, "Light Anti-quark Flavor Asymmetry in the Nucleon Sea and the Nuclear Dependence of Anti-quarks in Nuclei at the SeaQuest Experiment," $\mathrm{PhD}$ thesis, Rutgers, The State University of New Jersey, 2019.

[5] H. D. Politzer, "Reliable Perturbative Results for Strong Interactions?" Phys. Rev. Lett., vol. 30, pp. 1346-1349, 261973.

[6] D. J. Gross and F. Wilczek, "Ultraviolet Behavior of Non-Abelian Gauge Theories," Phys. Rev. Lett., vol. 30, pp. 1343-1346, 261973.

[7] D. E. S. John C. Collins and G. Sterman, "Factorization for short distance hadron-hadron scatterings," Nuclear Physics B, vol. 261, pp. $104-142,1985$.

[8] J. H. Christenson, G. S. Hicks, L. M. Lederman, P. J. Limon, B. G. Pope, and E. Zavattini, "Observation of Massive Muon Pairs in Hadron Collisions," Phys. Rev. Lett., vol. 25, pp. 1523-1526, 211970.

[9] S. D. Drell and T.-M. Yan, "Massive Lepton-Pair Production in Hadron-Hadron Collisions at High Energies," Phys. Rev. Lett., vol. 25, pp. 316-320, 51970.

[10] A. Khare, "The november j/psi revolution: Twenty-five years later," Curr.Sci. 77 (1999) 1210 ,

[11] P. P. Bhaduri, A. K. Chaudhuri, and S. Chattopadhyay, "J/psi production in proton induced collisions at fair," Phys. Rev., vol. C84, p. 054 914, 2011. arXiv: 1110.4268 [hep-ph].

[12] P. R.Vogt S.J.Brodsky, "Systematics of j/psi production in nuclear collisions," Nuclear Physics $B$, vol. 360, pp. $67-96,1991$.

[13] J. Peng, The case for j/psi at seaquest DOCID - 227-v1, [Online; accessed November 12, 2019]. 
[14] J. Peng, D. Jansen, and Y. Chen, "Probing ud asymmetry in the proton via quarkonium production," Physics Letters B, vol. 344, no. 1, pp. 1 -5, 1995.

[15] J. Kessler, "Search for Drell Yan in squareroot s = 41.6 GeV p-N Collisions at HERA-b," Jan. 2007.

[16] K. Lui, Flavor asymmetry in light quark sea and quark energy loss at fermilab e906/seaquest experiment DOCID - 1377-v1, [Online; accessed January 12, 2020].

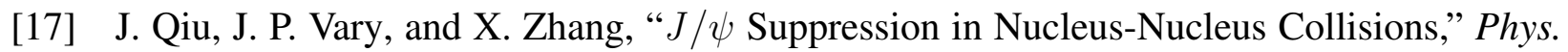
Rev. Lett., vol. 88, p. 232 301, 232002.

[18] P.-J. Lin, “Measurement of Quark Energy Loss in Cold Nuclear matter," PhD thesis, University of Colorado, 2017.

[19] T. Matsui and H. Satz, “ $J / \psi$ Suppression by Quark-Gluon Plasma Formation,” Phys. Lett., vol. B178, pp. 416-422, 1986.

[20] H. Satz, A Brief History of J/Psi Suppression, 1998. arXiv: hep-ph/9806319 [ hep-ph ].

[21] P. Foka and M. A. Janik, "An overview of experimental results from ultra-relativistic heavyion collisions at the cern lhc: Hard probes," Reviews in Physics, vol. 1, 172-194, 2016.

[22] A. Adare, C. Aidala, N. Ajitanand, Y. Akiba, R. Akimoto, H. Al-Bataineh, H. Al-Ta'ani, J. Alexander, K. Andrews, A. Angerami, and et al., "Cold-nuclear-matter effects on heavyquark production at forward and backward rapidity ind+aucollisions atsnn=200gev," Physical Review Letters, vol. 112, no. 25, 2014.

[23] C. Lourenco, R. Vogt, and H. K. Wohri, "Energy dependence of j/psi absorption in protonnucleus collisions," Journal of High Energy Physics, vol. 2009, no. 02, 014-014, 2009.

[24] F. Arleo, R. Kolevatov, S. Peigné, and M. Rustamova, "Centrality and pT dependence of $\mathrm{J} / \mathrm{psi}$ suppression in proton-nucleus collisions from parton energy loss," JHEP, vol. 05, p. 155, 2013. arXiv: 1304.0901 [hep-ph] .

[25] M. J. Leitch et al., "Measurement of differences between $J / \psi$ and $\psi$ ' Suppression in $p-A$ collisions," Phys. Rev. Lett., vol. 84, pp. 3256-3260, 152000.

[26] M. B. Johnson et al., "Energy loss of fast quarks in nuclei," Phys. Rev. Lett., vol. 86, pp. 4483 -4487, 202001.

[27] M. A. Vasiliev et al., "Parton Energy Loss Limits and Shadowing in Drell-Yan Dimuon Production,” Phys. Rev. Lett., vol. 83, pp. 2304-2307, 121999. 
[28] R. S. Towell et al., "Improved measurement of the dbar/ubar asymmetry in the nucleon sea," Phys. Rev. D, vol. 64, p. 052 002, 52001.

[29] C. Aidala et al., "The SeaQuest spectrometer at Fermilab," Nuclear Instruments and Methods in Physics Research Section A: Accelerators, Spectrometers, Detectors and Associated Equipment, vol. 930, pp. 49-63, 2019.

[30] Fermilab, Fermilab's Accelerator Complex, http: / / www . fnal.gov/pub/science/ particle-accelerators / accelerator-complex.html, [Online; accessed Feb 10, 2019].

[31] E. Garutti, Gaseous detectors- measurement of ionization position determination, http : / / www . desy . de / garutti / LECTURES / ParticleDetectorSS12 / L4_ gasDetectors.pdf, [Online; accessed January 21, 2020].

[32] S.-H. Shiu, J. Wu, R. E. McClellan, T.-H. Chang, W.-C. Chang, Y.-C. Chen, R. Gilman, K. Nakano, J.-C. Peng, and S.-Y. Wang, "FPGA-based trigger system for the Fermilab SeaQuest experimentz," Nuclear Instruments and Methods in Physics Research Section A: Accelerators, Spectrometers, Detectors and Associated Equipment, vol. 802, pp. 82 -88, 2015.

[33] S. Gorbunov and I. Kisel, CBM-SOFT-note-2006-001, [Online; accessed Mar 6, 2019].

[34] R. Kalman, "A New Approach to Linear Filtering and Prediction Problems," Journal of Basic Engineering, vol. 82(1), pp. 35-45, 1960.

[35] K. Nagai, Hit Cluster Removal DOCID - 1123, [Online; accessed Mar 3, 2019].

[36] K. Liu, Step-by-step walkthrough of kTracker DOCID - 1283, [Online; accessed Mar 3, 2019].

[37] M. H. Schub, et al., "Measurement of $J / \psi$ and $\psi$ ' production in $800 \mathrm{GeV} / \mathrm{c}$ proton-gold collisions," Phys. Rev. D, vol. 52, pp. 1307-1315, 31995.

[38] G. Moreno, Brown, et al., "Dimuon production in proton-copper collisions at sqrt(s)=38.8 gev," Phys. Rev. D, vol. 43, pp. 2815-2835, 91991.

[39] K. Nakano, Definition of ktrack.chisq target DOCID - 2339 - v1, [Online; accessed Mar 7, 2019].

[40] Y Chen, LD2 and LH2 Vent Pressure Analysis DOCID - 1453 - v3, [Online; accessed Mar 7, 2019].

[41] D. Geesaman, Handling the liquid target contamination and densities DOCID - 4993-v1, [Online; accessed Apr 12, 2019]. 
[42] K. Nagai, Ktracker-Efficiency on Extrapolation Method DOCID - 4456 - v1, [Online; accessed Apr 12, 2019]. 